Supporting information

\title{
Ni-Catalyzed Reductive anti-Arylative Cyclization of Alkynones
}

Zhijun Zhou, ${ }^{+}$Wenfeng Liu, ${ }^{+}$Wangqing Kong*

E-mail: wqkong@whu.edu.cn

The Center for Precision Synthesis, Institute for Advanced Studies, Wuhan

University, 299 Bayi Road, Wuhan, Hubei 430072, P. R. China 


\section{Contents}

1. General information S3

2. General procedures $\quad$ S4

$\begin{array}{lll}\text { 3. Additional experiments } & \mathrm{S} 7\end{array}$

4. General procedure for the synthesis of starting materials $\$ 9$

5. Characterization data of starting materials $\quad \mathrm{S} 11$

$\begin{array}{ll}\text { 6. Characterization data of products } & \$ 17\end{array}$

7. Copies of the ${ }^{1} \mathrm{H},{ }^{19} \mathrm{~F}$ and ${ }^{13} \mathrm{C}$ NMR spectra S41

8. References $\quad \mathbf{S 9 0}$ 


\section{General Information}

${ }^{1} \mathrm{H}$ and ${ }^{13} \mathrm{C}$ NMR data were recorded with Bruker ADVANCE III $(400 \mathrm{MHz})$ spectrometers. Chemical shifts are given in ppm. The spectra are calibrated to the residual ${ }^{1} \mathrm{H}$ and ${ }^{13} \mathrm{C}$ signals of the solvents. Multiplicities are abbreviated as follows: singlet (s), doublet (d), triplet (t), quartet (q), doublet-doublet (dd), quintet (quint), septet (sept), multiplet (m), and broad (b). ${ }^{19} \mathrm{~F}$ NMR spectra were recorded using $\mathrm{CFCl}_{3}$ as internal standard. Gas chromatography were determined with a SHIMADZU Nexis GC 2030 gas chromatography instrument with a FID detector. High resolution mass spectra (HRMS) were recorded on DIONEX UltiMate 3000 \& Bruker Compact TOF mass spectrometer. Enantiomeric excesses were determined with a SHIMADZU LC-20ADXR system using chiral stationary phase columns (DAICEL) by comparing the samples with the corresponding racemic samples. Column and elution details were specified in each entry. Optical rotations were measured with a polarimeter.

Materials and Methods: Unless otherwise stated, starting materials were purchased from commercial suppliers (Adamas-beta ${ }^{\circledR}$, Alfa, Aldrich and so on). All reactions dealing with air- or moisture-sensitive compounds were performed in the argon-filled glove box or by standard Schlenk techniques in oven-dried reaction vessels under nitrogen atmosphere. More sensitive compounds were stored in a desiccator or in a glove-box if required. Solvents were purchased in HPLC quality, degassed by purging thoroughly with nitrogen and dried over activated molecular sieves of appropriate size. Reactions were monitored by thin layer chromatography (TLC) using glass $0.25 \mathrm{~mm}$ silica gel plates. Compounds were visualized by UV-light at $254 \mathrm{~nm}$ and by dipping the plates in an aqueous potassium permanganate solution followed by heating. Flash column chromatography was performed over silica gel (230-400 mesh). 


\section{General Procedures}

\subsection{General Procedure for Racemic anti-Arylative Cyclization of Al-} kynones:
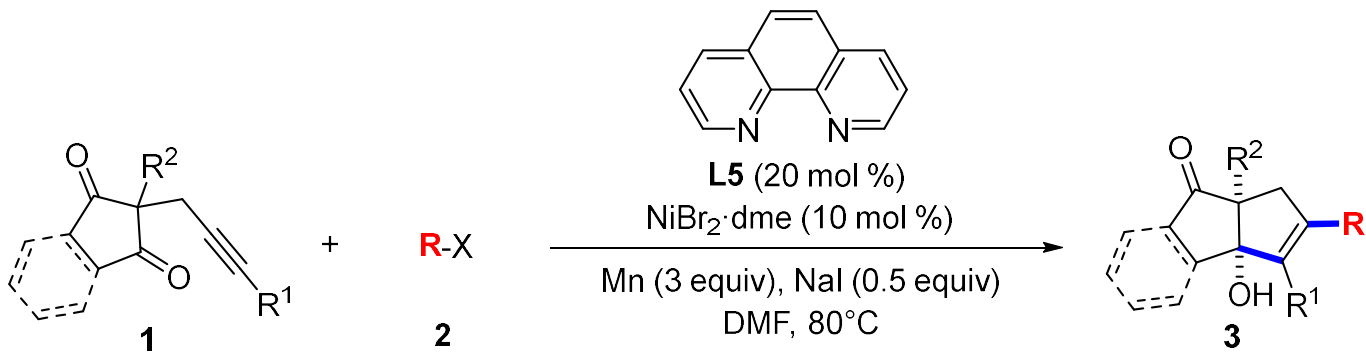

An oven-dried seal-tube equipped with a PTFE-coated stir bar was charged with alkynone 1 (0.2 mmol), organic halide 2 (4 equiv), phenanthroline L5 (20 mol\%), and brought into an Ar-filled glovebox. $\mathrm{NiBr} 2 \mathrm{dme}$ (10 mol\%), Mn dust (3 equiv), Nal (0.5 equiv) and anhydrous DMF (4 mL) was added. The seal-tube was sealed and stirring was continued in an aluminum bead bath preheated to $80{ }^{\circ} \mathrm{C}$ until the reaction was complete (monitored by TLC). The resulting mixture was quenched with saturated $\mathrm{NH}_{4} \mathrm{Cl}$ solution $(5 \mathrm{~mL})$ and further diluted with water $(10 \mathrm{~mL})$. The aqueous layer was extracted with ethyl acetate, and the combined organic layers were washed with brine, dried over anhydrous $\mathrm{Na}_{2} \mathrm{SO}_{4}$, filtered, and concentrated under vacuum. The residue was purified by chromatography on silica gel, eluting with ethyl acetate/petroleum ether $(1 / 20 \sim 1 / 5)$ to afford the desired product 3.

\subsection{Procedure for Asymmetric anti-Arylative Cyclization of Alkynone:}
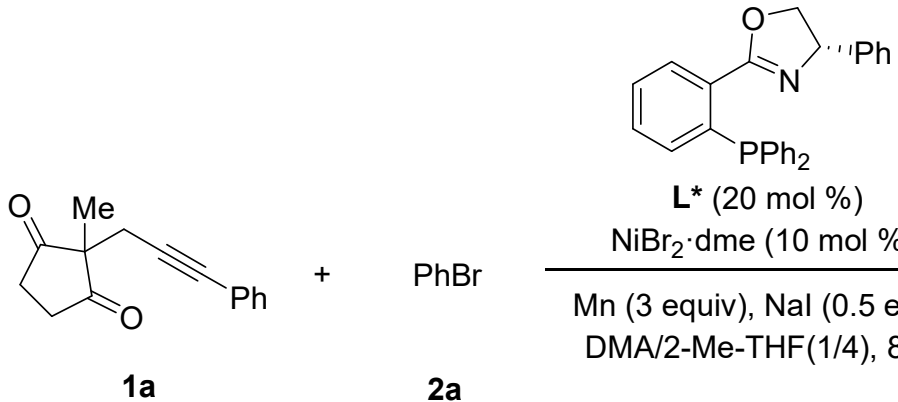

$$
\begin{aligned}
& L^{*}(20 \mathrm{~mol} \%) \\
& \underset{\mathrm{Mn} \text { (3 equiv), Nal }(0.5 \text { equiv })}{\stackrel{\mathrm{NiBr}_{2} \cdot \mathrm{dme}(10 \mathrm{~mol} \%)}{\longrightarrow}} \\
& \mathrm{DMA} / 2-\mathrm{Me}-\mathrm{THF}(1 / 4), 80^{\circ} \mathrm{C}
\end{aligned}
$$

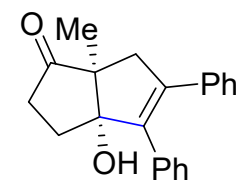

3aa, $65 \%$ yield, $81 \%$ ee

An oven-dried sealed tube equipped with a PTFE-coated stir bar was charged with $\mathrm{NiBr}_{2}$ dme (3.1 mg, $\left.10 \mathrm{~mol} \%, 0.01 \mathrm{mmol}\right),(\mathrm{S})-\mathrm{Ph}-\mathrm{Phox}\left(\mathrm{L}^{*}\right)(8.1 \mathrm{mg}, 20$ 
mol\%, $0.02 \mathrm{mmol})$, DMA $(0.2 \mathrm{~mL})$ and 2-Me-THF $(0.8 \mathrm{~mL})$ in an Ar-filled glovebox. This reaction mixture was stirred for 30 minutes in an aluminum bead bath preheated to $80^{\circ} \mathrm{C}$. Alkynone $1 \mathrm{a}(22.6 \mathrm{mg}, 0.1 \mathrm{mmol})$, phenyl bromide 2a (62.4 mg, 4 equiv, $0.4 \mathrm{mmol}$ ), $\mathrm{Mn}$ dust ( $16.5 \mathrm{mg}, 3$ equiv, $0.3 \mathrm{mmol}$ ), Nal (7.5 mg, 0.5 equiv, $0.05 \mathrm{mmol}), \mathrm{DMA}(0.2 \mathrm{~mL})$ and 2-Me-THF (0.8 mL) was then added. The sealed tube was sealed and stirring was continued at $80^{\circ} \mathrm{C}$ until the reaction was complete (monitored by TLC). The resulting mixture was quenched with saturated $\mathrm{NH}_{4} \mathrm{Cl}$ solution $(5 \mathrm{~mL})$ and further diluted with water $(10 \mathrm{~mL})$. The aqueous layer was extracted with ethyl acetate and the combined organic layers were washed with brine, dried over anhydrous $\mathrm{Na}_{2} \mathrm{SO}_{4}$, filtered, and concentrated under vacuum. The residue was purified by chromatography on silica gel, eluting with ethyl acetate/petroleum ether (1/20 1/5) to afford the desired product 3aa (19.8 $\mathrm{mg}, 65 \%$ yield).

\subsection{Procedure for anti-Arylative Cyclization of $1 \mathrm{a}$ on $1 \mathrm{mmol}$ Scale:}

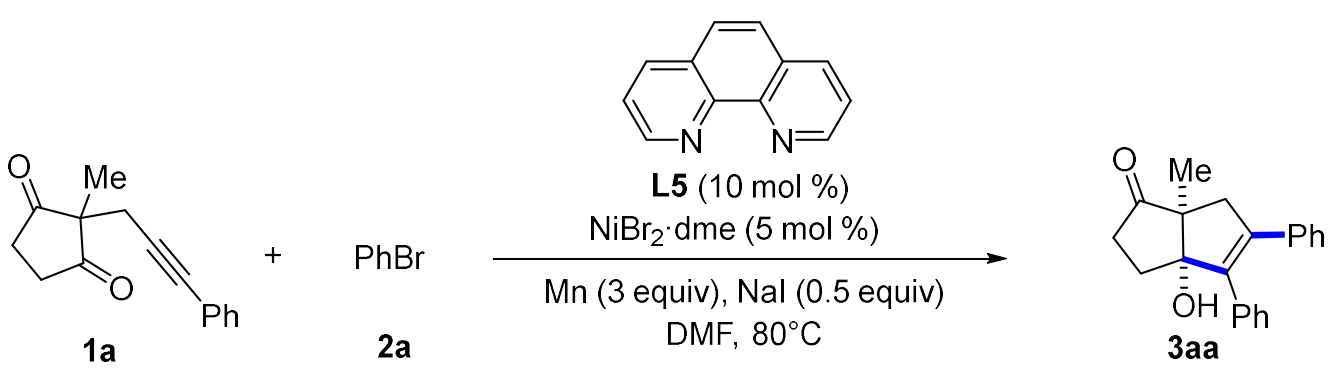

An oven-dried seal-tube equipped with a PTFE-coated stir bar was charged with alkynone 1a (226.0 mg, $1 \mathrm{mmol})$, bromobenzene $2(624.5 \mathrm{mg}, 4$ equiv, 4 mmol), phenanthroline L5 (18.5 mg, $10 \mathrm{~mol} \%, 0.1 \mathrm{mmol}$ ), and brought into an Ar-filled glovebox. $\mathrm{NiBr}_{2}$ dme (15.4 mg, $5 \mathrm{~mol} \%, 0.05 \mathrm{mmol}$ ), Mn dust (165.0 $\mathrm{mg}, 3$ equiv, $3 \mathrm{mmol})$, Nal $(75.0 \mathrm{mg}, 0.5$ equiv, $0.5 \mathrm{mmol}$ ) and anhydrous DMF $(20 \mathrm{~mL})$ was added. The seal-tube was sealed and stirring was continued in an aluminum bead bath preheated to $80{ }^{\circ} \mathrm{C}$ until the reaction was complete (monitored by TLC). The resulting mixture was quenched with saturated $\mathrm{NH}_{4} \mathrm{Cl}$ solution $(10 \mathrm{~mL})$ and further diluted with water $(50 \mathrm{~mL})$. The aqueous layer was extracted with ethyl acetate, and the combined organic layers were washed 
with brine, dried over anhydrous $\mathrm{Na}_{2} \mathrm{SO}_{4}$, filtered, and concentrated under vacuum. The residue was purified by chromatography on silica gel, eluting with ethyl acetate/petroleum ether $(1 / 20 \sim 1 / 5)$ to afford the desired product 3aa (152.5 mg, 50\% yield). 


\section{Additional experiments}

\subsection{Optimization of reaction conditions ${ }^{a}$}

\section{Table S1. Ligand screening}

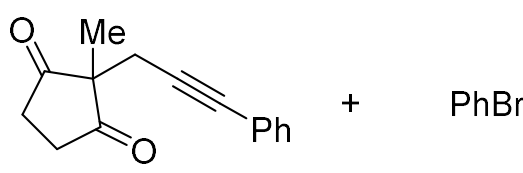

$1 \mathrm{a}$

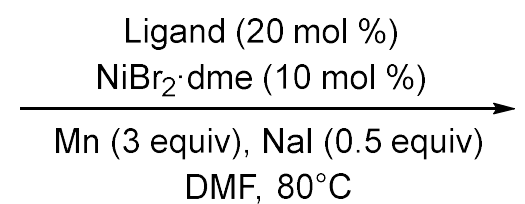

$\mathrm{DMF}, 80^{\circ} \mathrm{C}$

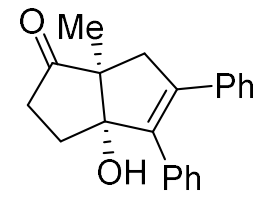

3aa<smiles>[R]c1ccc(P)c(C2=NC([R])CO2)c1</smiles>

L8: $R^{1}=H, R^{2}=P h$

L9: $R^{1}=H, R^{2}=B n$

L10: $R^{1}=H, R^{2}={ }^{i} P r$

L11: $R^{1}=\mathrm{CF}_{3}, \mathrm{R}^{2}={ }^{t_{\mathrm{Bu}}}$
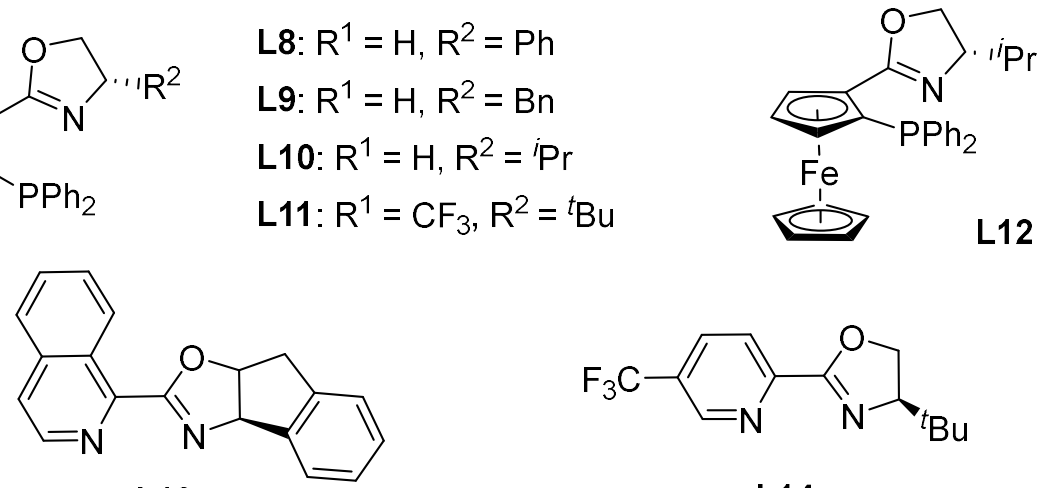

L13

L14

\begin{tabular}{cccc}
\hline entry & ligand & yield of 3aa $(\%)$ & ee of 3aa (\%) \\
\hline 1 & L8 & 44 & 30 \\
2 & L9 & 57 & 0 \\
3 & L10 & 48 & 22 \\
4 & L11 & 15 & 11 \\
5 & L12 & 40 & 0 \\
6 & L13 & 46 & 0 \\
7 & L14 & 41 & 0 \\
\hline
\end{tabular}


Table S2. Solvent screening

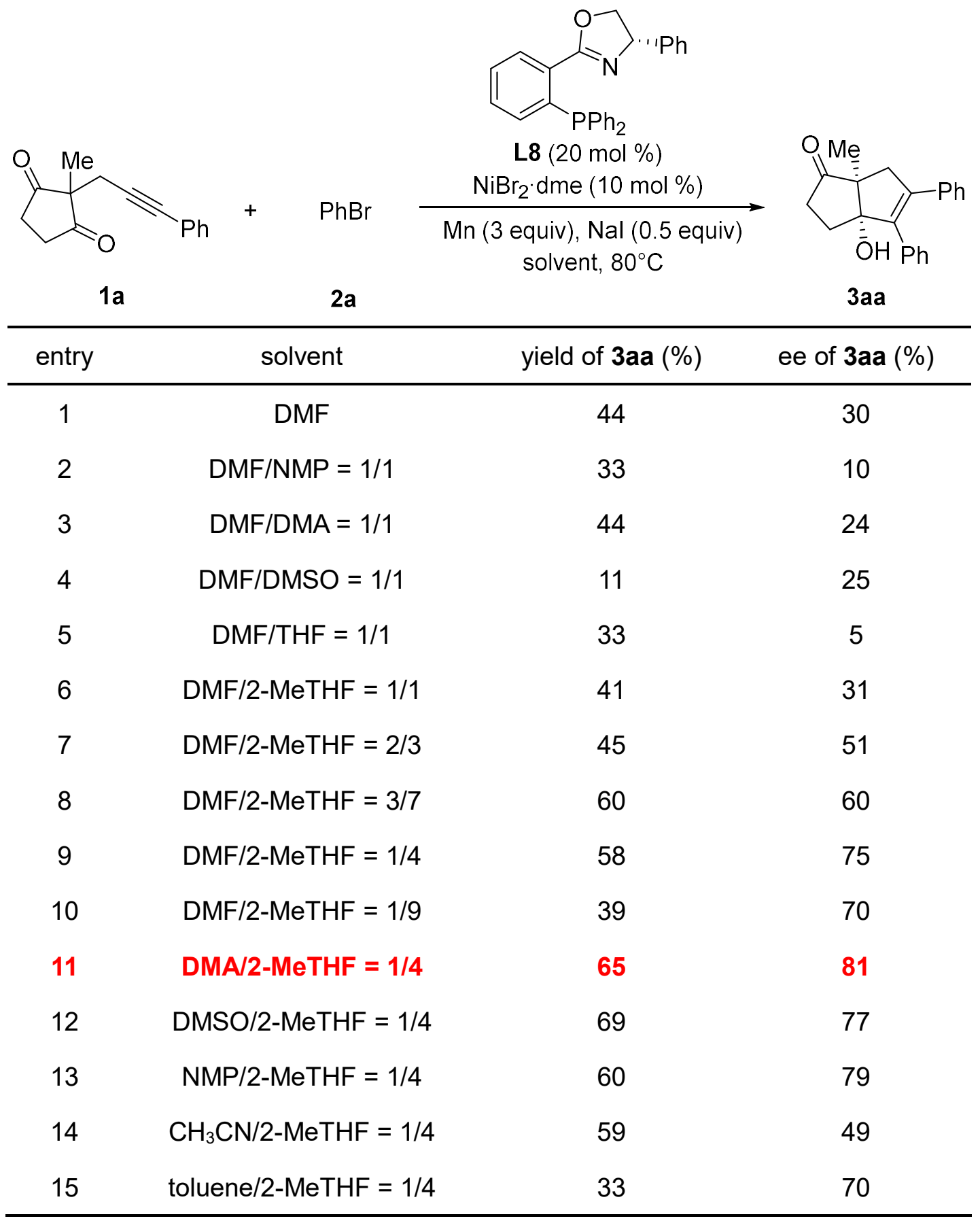




\section{General procedure for the synthesis of starting materials}

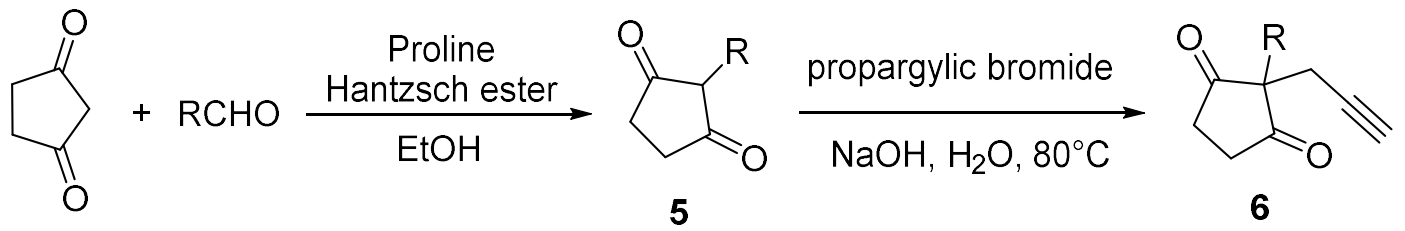

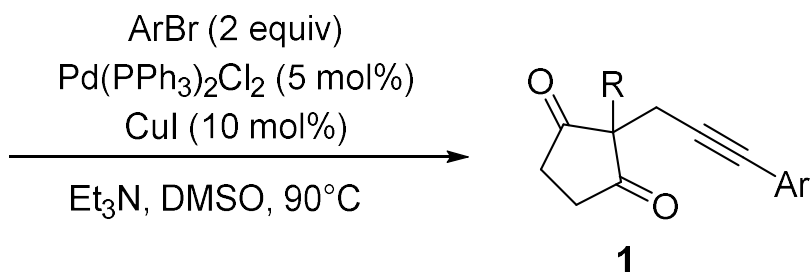

Proline (5 mol\%) was added to the mixture of the aldehyde ( 3 equiv), 1,3-dione $(0.3 \mathrm{mmol})$, Hantzsch ester (1 equiv) and $\mathrm{EtOH}(1 \mathrm{~mL})$. After the reaction was stirred at $25{ }^{\circ} \mathrm{C}$ for overnight, $\mathrm{H}_{2} \mathrm{O}(20 \mathrm{~mL})$ was added, and the mixture was extracted with EtOAc $(10 \mathrm{~mL})$ for three times. The combined organic phases were dried by anhydrous $\mathrm{NaSO}_{4}$, filtered, and concentrated in vacuo. The residue was purified by chromatography on silica gel, eluting with ethyl acetate/petroleum ether 1:20 2:1 (v/v) to afford the product $5 .{ }^{1}$

Adding $2 \mathrm{M}$ aqueous $\mathrm{NaOH}(20.0 \mathrm{mmol})$ to a slurry of $5(4.0 \mathrm{mmol})$ in water $(10 \mathrm{~mL})$. A bright red solution was formed when all of the solid had dissolved, then propargylic bromide $(8.0 \mathrm{mmol})$ was added. The mixture was stirred for $60 \mathrm{~h}$ in an aluminum bead bath preheated to $80^{\circ} \mathrm{C}$. The mixture was cooled to room temperature, EtOAc $(10 \mathrm{~mL})$ was added, and the mixture was extracted with EtOAc $(3 \times 10 \mathrm{~mL})$. The combined organic phases were dried $\mathrm{NaSO}_{4}$, filtered, and concentrated in vacuo. The residue was purified by chromatography on silica gel, eluting with ethyl acetate/petroleum ether 1:20 2:1 (v/v) to afford the product $6 .^{2}$

Alkyne $6(10.0 \mathrm{mmol})$ and aryl bromide $(20.0 \mathrm{mmol})$ was added to a solution of $\mathrm{Pd}\left(\mathrm{PPh}_{3}\right)_{2} \mathrm{Cl}_{2}(0.5 \mathrm{mmol})$, Cul $(1.0 \mathrm{mmol})$, and $\mathrm{Et}_{3} \mathrm{~N}(20.0 \mathrm{mmol})$ in anhydrous DMSO (24 mL). The mixture was stirred for $3 \mathrm{~h}$ in an aluminum bead bath preheated to $90^{\circ} \mathrm{C}$. When the reaction was cooled to room temperature, 
water $(50 \mathrm{~mL})$ was added, and the mixture was extracted with $\mathrm{Et}_{2} \mathrm{O}(50 \mathrm{~mL})$. The organic phase was washed with $10 \%$ aqueous $\mathrm{HCl}(3 \times 20 \mathrm{~mL})$, dried $\mathrm{Na}_{2} \mathrm{SO}_{4}$, filtered, and concentrated in vacuo. The residue was purified by chromatography on silica gel, eluting with ethyl acetate/petroleum ether 1:20 1:5 (v/v) to afford the alkynone $1 .^{3}$ 


\section{Characterization data of starting materials}

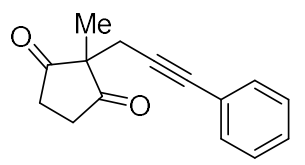

$1 \mathrm{a}$

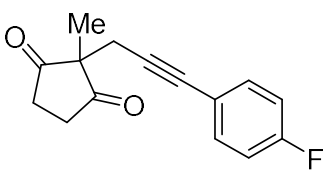

$1 \mathrm{e}$

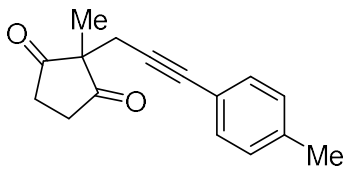

$1 b$

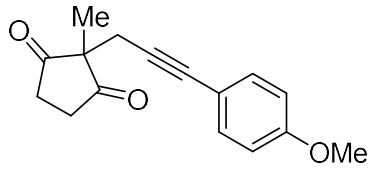

$1 \mathrm{c}$

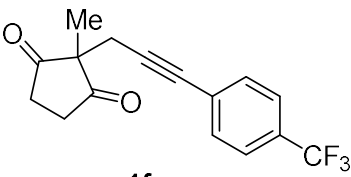

$1 f$

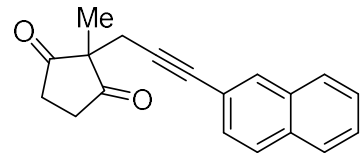

$1 \mathrm{j}$

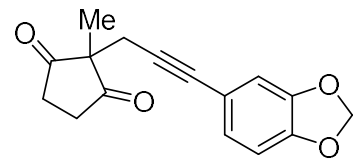

$1 \mathrm{k}$

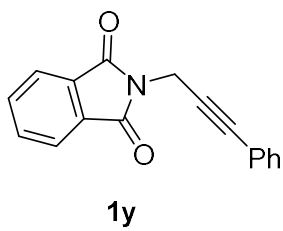

$1 \mathrm{~s}$
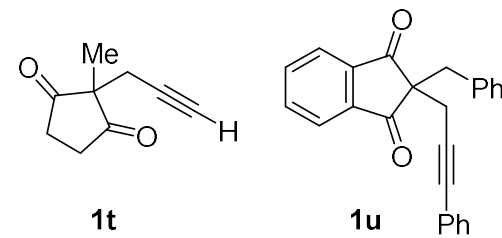

$1 \mathrm{t}$

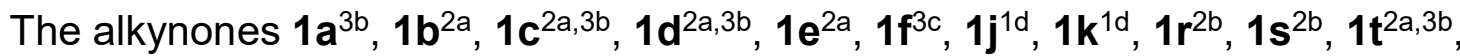
$1 \mathbf{u}^{3 \mathrm{c}}$ and $\mathbf{1} \mathbf{y}^{4}$ were prepared according to previously reported procedures.

ethyl 4-(3-(1-methyl-2,5-dioxocyclopentyl)prop-1-yn-1-yl)benzoate (1g)<smiles>CCOC(=O)c1ccc(C#CCC2(C)C(=O)CCC2=O)cc1</smiles>

Chemical Formula: $\mathrm{C}_{18} \mathrm{H}_{18} \mathrm{O}_{4}$

Exact Mass: 298.1205

1g was prepared according to general procedure 4 and was purified by silica gel column chromatography (PE/EA $=5 / 1)$ as yellow solid $(2.3 \mathrm{~g}, 78 \%$ yield). m.p. $55-57{ }^{\circ} \mathrm{C} .{ }^{1} \mathrm{H}$ NMR $\left(600 \mathrm{MHz}, \mathrm{CDCl}_{3}\right) \delta$ 7.96-7.92 (m, 2H), 7.38-7.35 (m, 2H), 4.36 (q, $J=7.1 \mathrm{~Hz}, 2 \mathrm{H}), 2.90-2.78(\mathrm{~m}, 4 \mathrm{H}), 2.71(\mathrm{~s}, 2 \mathrm{H}), 1.38(\mathrm{t}, J=7.1$ $\mathrm{Hz}, 3 \mathrm{H}), 1.19$ (s, 3H); ${ }^{13} \mathrm{C}$ NMR (151 MHz, $\left.\mathrm{CDCl}_{3}\right) \delta$ 215.2, 165.9, 131.5, 130.0, 129.4, 127.1, 87.1, 82.2, 61.1, 55.4, 35.8, 25.5, 19.2, 14.3; HRMS: (ESI) calcd for $\mathrm{C}_{18} \mathrm{H}_{19} \mathrm{O}_{4}{ }^{+}[\mathrm{M}+\mathrm{H}]^{+} 299.1278$; found 299.1276.

2-methyl-2-(3-(4-(4,4,5,5-tetramethyl-1,3,2-dioxaborolan-2-yl)phenyl)prop-2-y 


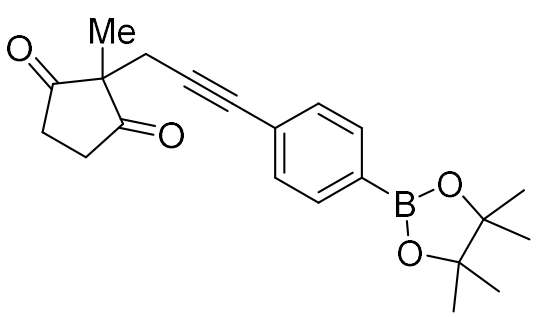

Chemical Formula: $\mathrm{C}_{21} \mathrm{H}_{25} \mathrm{BO}_{4}$

Exact Mass: 352.1846

1h was prepared according to general procedure 4 and was purified by silica gel column chromatography $(P E / E A=5 / 1)$ as yellow solid $(2.2 \mathrm{~g}, 62 \%$ yield $)$. m.p. $98-100{ }^{\circ} \mathrm{C} .{ }^{1} \mathrm{H}$ NMR $\left(600 \mathrm{MHz}, \mathrm{CDCl}_{3}\right) \delta$ 7.71-7.68 (m, 2H), 7.32-7.29 (m, 2H), $2.82(\mathrm{~s}, 4 \mathrm{H}), 2.69(\mathrm{~s}, 2 \mathrm{H}), 1.32(\mathrm{~s}, 12 \mathrm{H}), 1.17(\mathrm{~s}, 3 \mathrm{H}) ;{ }^{13} \mathrm{C}$ NMR $(151 \mathrm{MHz}$, $\left.\mathrm{CDCl}_{3}\right) \delta 215.5,134.5,130.8,125.2,85.3,83.9,83.0,55.4,35.9,25.9,24.8$, 18.9; HRMS: (ESI) calcd for $\mathrm{C}_{21} \mathrm{H}_{26} \mathrm{BO}_{4}{ }^{+}[\mathrm{M}+\mathrm{H}]^{+} 353.1919$; found 353.1910.

3-(3-(1-methyl-2,5-dioxocyclopentyl)prop-1-yn-1-yl)benzonitrile (1i)<smiles>CC1(CC#Cc2cccc(C#N)c2)C(=O)CCC1=O</smiles>

Chemical Formula: $\mathrm{C}_{16} \mathrm{H}_{13} \mathrm{NO}_{2}$ Exact Mass: 251.0946

1i was prepared according to general procedure 4 and was purified by silica gel column chromatography $(\mathrm{PE} / \mathrm{EA}=4 / 1)$ as yellow solid $(2.0 \mathrm{~g}, 80 \%$ yield $)$. m.p. 84-86 ${ }^{\circ} \mathrm{C} .{ }^{1} \mathrm{H}$ NMR $\left(600 \mathrm{MHz}, \mathrm{CDCl}_{3}\right) \delta$ 7.61-7.59 (m, 1H), 7.59-7.53 (m, 2H), 7.42-7.38 (m, 1H), 2.92-2.76 (m, 4H), $2.71(\mathrm{~s}, 2 \mathrm{H}), 1.21(\mathrm{~s}, 3 \mathrm{H}) .{ }^{13} \mathrm{C}$ NMR $\left(151 \mathrm{MHz}, \mathrm{CDCl}_{3}\right) \delta 214.8,135.7,134.9,131.5,129.2,124.2,117.9,112.8$, 86.9, 80.5, 55.3, 35.7, 25.0, 19.4; HRMS: (ESI) calcd for $\mathrm{C}_{16} \mathrm{H}_{14} \mathrm{NO}_{2}{ }^{+}[\mathrm{M}+\mathrm{H}]^{+}$ 252.1019; found 252.1019.

2-(3-(2,3-dihydrobenzo[b][1,4]dioxin-6-yl)prop-2-yn-1-yl)-2-methylcyclopentan e-1,3-dione (1I) 


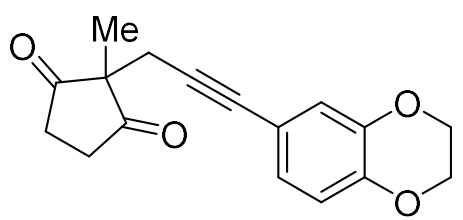

Chemical Formula: $\mathrm{C}_{17} \mathrm{H}_{16} \mathrm{O}_{4}$

Exact Mass: 284.1049

1I was prepared according to general procedure 4 and was purified by silica gel column chromatography $(\mathrm{PE} / \mathrm{EA}=5 / 1)$ as yellow solid $(2.0 \mathrm{~g}, 72 \%$ yield $)$. m.p. $68-70{ }^{\circ} \mathrm{C} .{ }^{1} \mathrm{H}$ NMR $\left(600 \mathrm{MHz}, \mathrm{CDCl}_{3}\right) \delta$ 6.84-6.82 (m, 1H), 6.82-6.79 (m, 1H), 6.75-6.73 (m, 1H), 4.25-4.21 (m, 4H), 2.86-2.77 (m, 4H), $2.64(\mathrm{~s}, 2 \mathrm{H})$, 1.15 (s, 3H); ${ }^{13} \mathrm{C}$ NMR (151 MHz, $\left.\mathrm{CDCl}_{3}\right) \delta 215.8,144.1,143.2,125.1,120.4$, 117.3, 115.3, 82.5, 82.2, 64.4, 64.2, 55.5, 36.0, 26.1, 18.8; HRMS: (ESI) calcd for $\mathrm{C}_{17} \mathrm{H}_{17} \mathrm{O}_{4}{ }^{+}[\mathrm{M}+\mathrm{H}]^{+} 285.1121$; found 285.1127 .

2-methyl-2-(3-(1-methyl-1H-indol-5-yl)prop-2-yn-1-yl)cyclopentane-1,3-dione $(1 \mathrm{~m})$

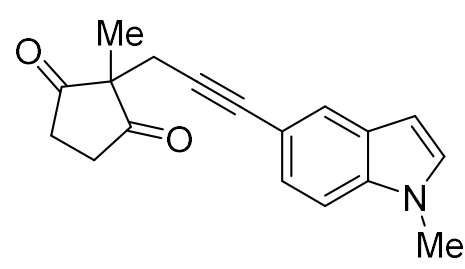

Chemical Formula: $\mathrm{C}_{18} \mathrm{H}_{17} \mathrm{NO}_{2}$

Exact Mass: 279.1259

$1 \mathrm{~m}$ was prepared according to general procedure 4 and was purified by silica gel column chromatography (PE/EA $=5 / 1)$ as yellow solid $(1.4 \mathrm{~g}, 52 \%$ yield). m.p. 89-91 ${ }^{\circ} \mathrm{C} .{ }^{1} \mathrm{H}$ NMR $\left(600 \mathrm{MHz}, \mathrm{CDCl}_{3}\right)$ ठ 7.64-7.61 (m, 1H), 7.22-7.20 (m, $1 \mathrm{H})$, 7.19-7.16 (m, 1H), 7.07-7.04 (m, 1H), 6.45-6.41 (m, 1H), $3.77(\mathrm{~s}, 3 \mathrm{H})$, 2.93-2.76 (m, 4H), $2.70(\mathrm{~s}, 2 \mathrm{H}), 1.17(\mathrm{~s}, 3 \mathrm{H}) ;{ }^{13} \mathrm{C} \mathrm{NMR}\left(151 \mathrm{MHz}, \mathrm{CDCl}_{3}\right) \delta$ 216.1, 136.3, 129.8, 128.2, 125.0, 124.7, 113.0, 109.2, 101.1, 84.4, 81.1, 55.7, 36.1, 32.9, 26.6, 18.6; HRMS: (ESI) calcd for $\mathrm{C}_{18} \mathrm{H}_{18} \mathrm{NO}_{2}{ }^{+}[\mathrm{M}+\mathrm{H}]^{+} 280.1332$; found 280.1333 .

2-(3-(dibenzo[b, d]thiophen-2-yl)prop-2-yn-1-yl)-2-methylcyclopentane-1,3-dion 
e $(1 n)$

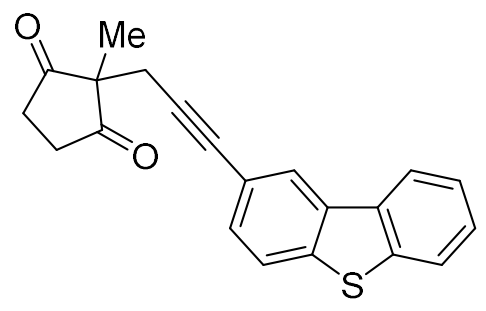

Chemical Formula: $\mathrm{C}_{21} \mathrm{H}_{16} \mathrm{O}_{2} \mathrm{~S}$

Exact Mass: 332.0871

1n was prepared according to general procedure 4 and was purified by silica gel column chromatography (PE/EA $=5 / 1)$ as yellow solid $(2.5 \mathrm{~g}, 75 \%$ yield). m.p. 100-102 ' C. ${ }^{1} \mathrm{H}$ NMR (600 MHz, $\left.\mathrm{CDCl}_{3}\right)$ ס 8.13-8.10 (m, 2H), 7.85-7.82 (m, 1H), 7.76-7.73 (m, 1H), 7.49-7.45 (m, 2H), 7.41-7.38 (m, 1H), 2.90-2.84 (m, 4H), 2.75 (s, 2H), 1.21 (s, 3H); ${ }^{13} \mathrm{C}$ NMR (151 MHz, $\left.\mathrm{CDCl}_{3}\right) \delta$ 215.6, 139.7, $139.5,135.5,134.74,129.7,127.2$, 124.7, 124.6, 122.8, 122.7, 121.7, 118.5, 83.9, 82.9, 55.5, 36.0, 25.9, 19.0; HRMS: (ESI) calcd for $\mathrm{C}_{21} \mathrm{H}_{17} \mathrm{O}_{2} \mathrm{~S}^{+}[\mathrm{M}+\mathrm{H}]^{+}$ 333.0944; found 333.0944.

2-(3-(dibenzo[b, d]furan-2-yl)prop-2-yn-1-yl)-2-methylcyclopentane-1,3-dione (10)

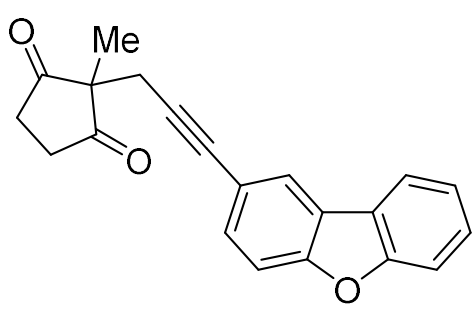

Chemical Formula: $\mathrm{C}_{21} \mathrm{H}_{16} \mathrm{O}_{3}$ Exact Mass: 316.1099

10 was prepared according to general procedure 4 and was purified by silica gel column chromatography $(P E / E A=5 / 1)$ as yellow solid $(2.3 \mathrm{~g}, 72 \%$ yield $)$.

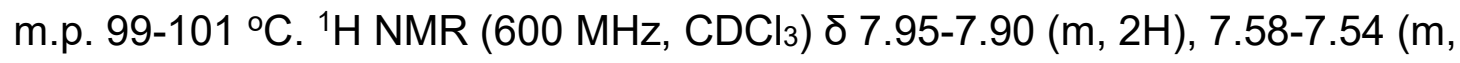
1H), 7.50-7.45 (m, 2H), 7.43-7.41 (m, 1H), 7.39-7.33 (m, 1H), 2.92-2.82 (m, 4H), 2.73 (s, 2H), 1.21 (s, 3H); ${ }^{13} \mathrm{C}$ NMR (151 MHz, $\left.\mathrm{CDCl}_{3}\right) \delta$ 215.6, 156.5, $155.8,130.8,127.7,124.4,124.1,123.4,123.1,120.8,117.0,111.8,111.8$, 
82.9, 82.9, 55.6, 36.0, 26.0, 19.0; HRMS: (ESI) calcd for $\mathrm{C}_{21} \mathrm{H}_{17} \mathrm{O}_{3}{ }^{+}[\mathrm{M}+\mathrm{H}]^{+}$ 317.1172; found 317.1171.

2-(3-(6-methoxypyridin-3-yl)prop-2-yn-1-yl)-2-methylcyclopentane-1,3-dione (1p)

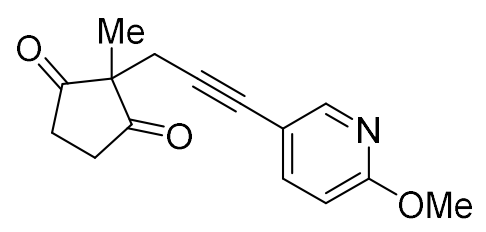

Chemical Formula: $\mathrm{C}_{15} \mathrm{H}_{15} \mathrm{NO}_{3}$

Exact Mass: 257.1052

$1 \mathrm{p}$ was prepared according to general procedure 4 and was purified by silica gel column chromatography ( $P E / E A=5 / 1)$ as yellow solid $(1.7 \mathrm{~g}, 68 \%$ yield). m.p. $50-52{ }^{\circ} \mathrm{C} .{ }^{1} \mathrm{H}$ NMR $\left(600 \mathrm{MHz}, \mathrm{CDCl}_{3}\right)$ ठ 8.15-8.12 (m, 1H), 7.49-7.45 (m, 1H), 6.66-6.63 (m, 1H), $3.91(\mathrm{~s}, 3 \mathrm{H}), 2.87-2.77(\mathrm{~m}, 4 \mathrm{H}), 2.68(\mathrm{~s}, 2 \mathrm{H}), 1.17$ (s, $3 \mathrm{H}) ;{ }^{13} \mathrm{C}$ NMR $\left(151 \mathrm{MHz}, \mathrm{CDCl}_{3}\right) \delta 215.4,163.4,150.0,141.1,112.3,110.6$, 85.4, 79.6, 55.4, 53.6, 35.9, 25.7, 19.0; HRMS: (ESI) calcd for $\mathrm{C}_{15} \mathrm{H}_{16} \mathrm{NO}_{3}{ }^{+}[\mathrm{M}+\mathrm{H}]^{+} 258.1125$; found 258.1124.

2-methyl-2-(3-((8R,9S,13S, 14S)-13-methyl-17-oxo-7,8,9,11,12,13,14, 15,16,17 -decahydro-6H-cyclopenta[a]phenanthren-3-yl)prop-2-yn-1-yl)cyclopentane-1, 3-dione (1q)

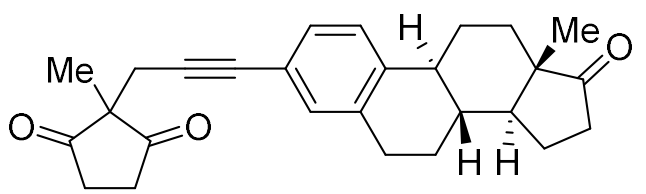

Chemical Formula: $\mathrm{C}_{27} \mathrm{H}_{30} \mathrm{O}_{3}$

Exact Mass: 402.2195

1q was prepared according to general procedure 4 and was purified by silica gel column chromatography $(\mathrm{PE} / \mathrm{EA}=5 / 1)$ as yellow solid $(2.1 \mathrm{~g}, 52 \%$ yield $)$. m.p. $177-179{ }^{\circ} \mathrm{C} .{ }^{1} \mathrm{H} N M R\left(600 \mathrm{MHz}, \mathrm{CDCl}_{3}\right) \delta$ 7.21-7.18 (m, 1H), 7.12-7.09 (m, $1 \mathrm{H}), 7.08-7.06(\mathrm{~m}, 1 \mathrm{H}), 2.88-2.79(\mathrm{~m}, 6 \mathrm{H}), 2.67(\mathrm{~s}, 2 \mathrm{H}), 2.53-2.47(\mathrm{~m}, 1 \mathrm{H})$, 
2.40-2.36 (m, 1H), 2.30-2.24 (m, 1H), 2.18-2.10 (m, 1H), 2.08-2.03 (m, 1H), 2.03-1.98 $(\mathrm{m}, 1 \mathrm{H}), 1.98-1.93(\mathrm{~m}, 1 \mathrm{H}), 1.66-1.60(\mathrm{~m}, 1 \mathrm{H}), 1.58-1.47(\mathrm{~m}, 4 \mathrm{H})$, 1.46-1.40 (m, 1H), $1.16(\mathrm{~s}, 3 \mathrm{H}), 0.90(\mathrm{~s}, 3 \mathrm{H}) ;{ }^{13} \mathrm{C} \mathrm{NMR}\left(151 \mathrm{MHz}, \mathrm{CDCl}_{3}\right) \delta$ 220.7, 215.7, 140.3, 136.6, 132.1, 128.9, 125.4, 119.8, 83.20 82.9, 55.5, 50.4, 47.9, 44.4, 37.9, 36.0, 35.8, 31.5, 29.0, 26.3, 26.1, 25.5, 21.5, 18.8, 13.8; HRMS: (ESI) calcd for $\mathrm{C}_{27} \mathrm{H}_{31} \mathrm{O}_{3}{ }^{+}[\mathrm{M}+\mathrm{H}]^{+} 404.2268$; found 404.2278.

2,5,5-trimethyl-2-(3-phenylprop-2-yn-1-yl)cyclohexane-1,3-dione (1v)

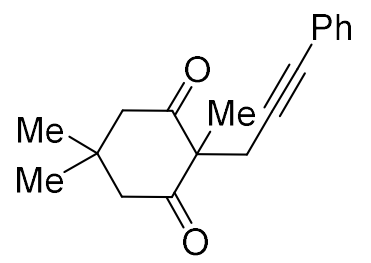

Chemical Formula: $\mathrm{C}_{18} \mathrm{H}_{20} \mathrm{O}_{2}$

Exact Mass: 268.1463

1v was prepared according to general procedure 4 and was purified by silica gel column chromatography ( $P E / E A=5 / 1)$ as yellow solid $(0.8 \mathrm{~g}, 32 \%$ yield $)$. m.p. 67-69 ${ }^{\circ} \mathrm{C} .{ }^{1} \mathrm{H}$ NMR $\left(600 \mathrm{MHz}, \mathrm{CDCl}_{3}\right) \delta$ 7.33-7.29 (m, 2H), 7.27-7.23 (m, $3 \mathrm{H}), 2.84(\mathrm{~s}, 2 \mathrm{H}), 2.66(\mathrm{q}, 4 \mathrm{H}), 1.37(\mathrm{~s}, 3 \mathrm{H}), 1.05(\mathrm{~s}, 3 \mathrm{H}), 1.04(\mathrm{~s}, 3 \mathrm{H}) ;{ }^{13} \mathrm{C}$ NMR $\left(151 \mathrm{MHz}, \mathrm{CDCl}_{3}\right) \delta 208.8,131.5,128.1,127.9,123.1,85.9,83.1,63.4$, 51.8, 30.5, 29.3, 27.9, 25.8, 22.2; HRMS: (ESI) calcd for $\mathrm{C}_{18} \mathrm{H}_{21} \mathrm{O}_{2}{ }^{+}[\mathrm{M}+\mathrm{H}]^{+}$ 269.1536; found 269.1530 . 


\section{Characterization data of products}

3a-hydroxy-6a-methyl-4,5-diphenyl-3,3a,6,6a-tetrahydropentalen-1(2H)-one (3aa)<smiles>CC12CCCC1(O)C(c1ccccc1)=C(c1ccccc1)C(=O)C2</smiles>

Chemical Formula: $\mathrm{C}_{21} \mathrm{H}_{20} \mathrm{O}_{2}$

Exact Mass: 304.1463

3aa was prepared according to general procedure 2.1 using $\mathbf{1 a}$ and $\mathbf{2 a}$ and was purified by silica gel column chromatography $(P E / E A=7 / 1)$ to obtain 3aa as yellow oil (21.0 mg, 69\% yield). ${ }^{1} \mathrm{H}$ NMR (400 MHz, $\left.\mathrm{CDCl}_{3}\right) \delta$ 7.31-7.27 (m, 3H), 7.26-7.23 (m, 2H), 7.18-7.13 (m, 3H), 7.13-7.09 (m, 2H), 3.25 (d, J = 17.1 $\mathrm{Hz}, 1 \mathrm{H}), 2.72(\mathrm{~d}, J=17.1 \mathrm{~Hz}, 1 \mathrm{H}), 2.48-2.38(\mathrm{~m}, 1 \mathrm{H}), 2.20-2.08(\mathrm{~m}, 2 \mathrm{H})$, 2.07-1.89 (m, 2H), $1.22(\mathrm{~s}, 3 \mathrm{H}) ;{ }^{13} \mathrm{C}$ NMR (101 MHz, CDCl 3 ) $\delta$ 222.1, 142.6, 140.2, 135.9, 135.7, 129.1, 128.7, 128.2, 128.0, 127.6, 92.9, 56.4, 44.9, 36.7, 29.2, 15.6; HRMS: (ESI) calcd for $\mathrm{C}_{21} \mathrm{H}_{20} \mathrm{NaO}_{2}{ }^{+}[\mathrm{M}+\mathrm{Na}]^{+} 327.1355$; found 327.1346.

The enantiomeric purity was established by HPLC analysis using a chiral column: AD-H column, $30{ }^{\circ} \mathrm{C}, n$-Hexane/i-Propanol $=85 / 15$ as eluent, $254 \mathrm{~nm}$, $1 \mathrm{~mL} / \mathrm{min}, \mathrm{tR}=6 \mathrm{~min}$ (minor), $7 \mathrm{~min}$ (major); $81 \%$ ee.

Optical Rotation: $[\alpha]_{D}^{32}-5.1$ (c 1.3, $\left.{ }^{i} \mathrm{PrOH}\right)$ for $81 \%$ ee. 
〈色谱图〉

$\mathrm{mAU}$

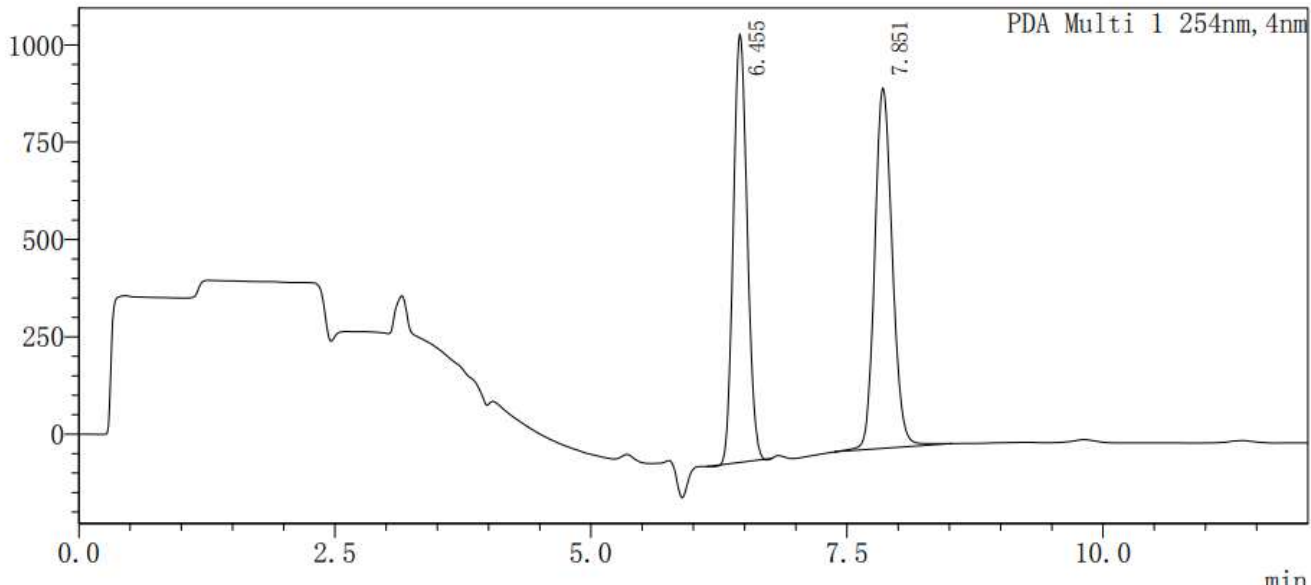

〈峰表〉

PDA Ch1 254nm

\begin{tabular}{|r|r|r|r|r|r|r|r|}
\hline 峰号 & 保留时间 & \multicolumn{1}{|c|}{ 面积 } & 高度 & \multicolumn{1}{|c|}{ 浓度 } & 浓度单位 & 标记 & 化合物名 \\
\hline 1 & 6.455 & 10345446 & 1100872 & 0.000 & & $\mathrm{M}$ & \\
\hline 2 & 7.851 & 11071927 & 926115 & 0.000 & & $\mathrm{M}$ & \\
\hline 总计 & & 21417373 & 2026987 & & & & \\
\hline
\end{tabular}

〈色谱图〉

$\mathrm{mAU}$

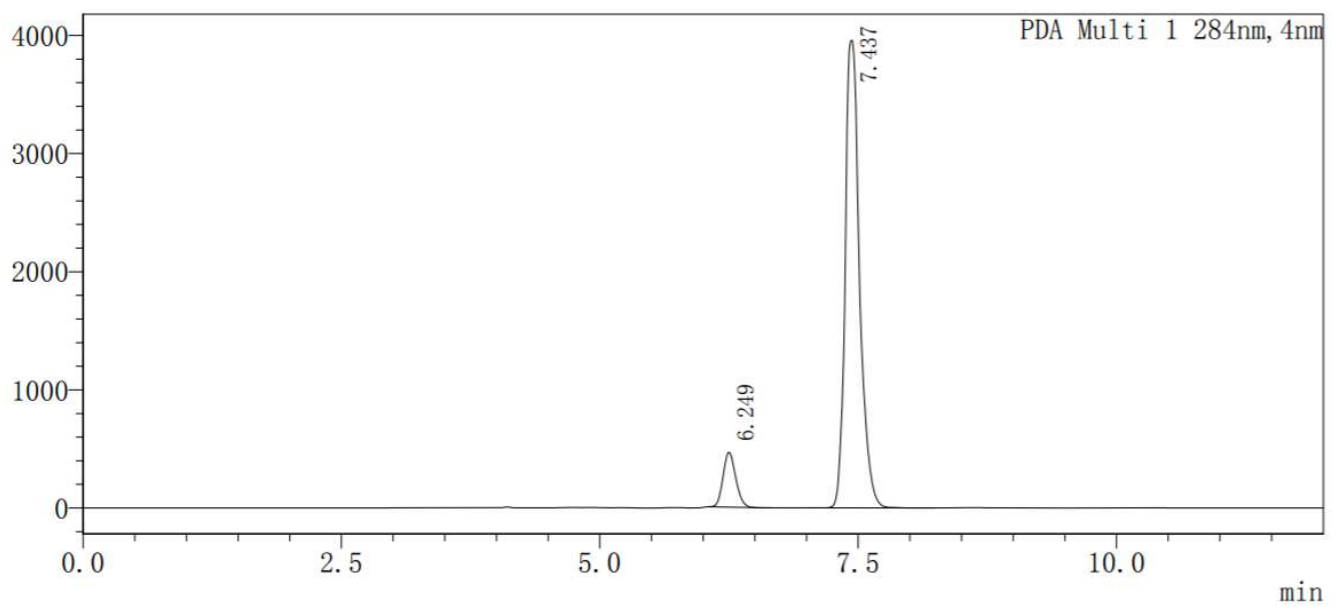

〈峰表〉

PDA Ch1 284nm

\begin{tabular}{|r|r|r|r|r|r|r|r|}
\hline \multicolumn{1}{|c|}{ 峰号 } & 保留时间 & \multicolumn{1}{|c|}{ 面积 } & \multicolumn{1}{c|}{ 高度 } & \multicolumn{1}{c|}{ 浓度 } & 浓度单位 & 标记 & 化合物名 \\
\hline 1 & 6.249 & 3935975 & 465103 & 0.000 & & $\mathrm{M}$ & \\
\hline 2 & 7.437 & 38180234 & 3958593 & 0.000 & & $\mathrm{M}$ & \\
\hline 总计 & & 42116208 & 4423695 & & & & \\
\hline
\end{tabular}


3a-hydroxy-5-(4-methoxyphenyl)-6a-methyl-4-phenyl-3,3a,6,6a-tetrahydropen talen-1(2H)-one (3ab)

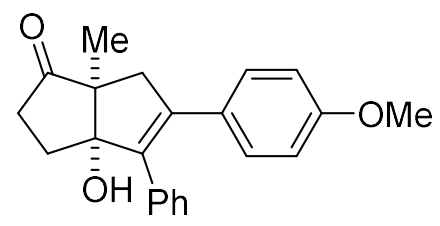

Chemical Formula: $\mathrm{C}_{22} \mathrm{H}_{22} \mathrm{O}_{3}$

Exact Mass: 334.1569

3ab was prepared according to general procedure 2.1 using $\mathbf{1 a}$ and $\mathbf{2 b}$ and was purified by silica gel column chromatography $(P E / E A=5 / 1)$ to obtain 3ab as white solid (56.1 mg, 84\% yield). ${ }^{1} \mathrm{H}$ NMR $\left(400 \mathrm{MHz}, \mathrm{CDCl}_{3}\right) \delta 7.31-7.22(\mathrm{~m}$, $5 \mathrm{H}), 7.06-7.00(\mathrm{~m}, 2 \mathrm{H}), 6.69-6.62(\mathrm{~m}, 2 \mathrm{H}), 3.72(\mathrm{~s}, 3 \mathrm{H}), 3.21(\mathrm{~d}, J=17.0 \mathrm{~Hz}$, $1 \mathrm{H}), 2.66(\mathrm{~d}, J=17.0 \mathrm{~Hz}, 1 \mathrm{H}), 2.45-2.34(\mathrm{~m}, 1 \mathrm{H}), 2.16-1.98(\mathrm{~m}, 3 \mathrm{H}), 1.95-1.86$ (m, $1 \mathrm{H}), 1.20(\mathrm{~s}, 3 \mathrm{H}) ;{ }^{13} \mathrm{C}$ NMR $\left(101 \mathrm{MHz}, \mathrm{CDCl}_{3}\right) \delta 222.1,159.1,141.8,138.4$, 136.2, 129.9, 129.0, 128.6, 127.9, 127.3, 113.4, 92.7, 56.2, 55.1, 44.5, 36.5, 28.9, 15.5; HRMS: (ESI) calcd for $\mathrm{C}_{22} \mathrm{H}_{22} \mathrm{NaO}_{3}{ }^{+}[\mathrm{M}+\mathrm{Na}]^{+} 357.1461$; found 357.1468 .

5-(4-(dimethylamino)phenyl)-3a-hydroxy-6a-methyl-4-phenyl-3,3a,6,6a-tetrahy dropentalen-1(2H)-one (3ac)

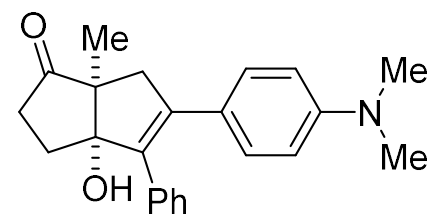

Chemical Formula: $\mathrm{C}_{23} \mathrm{H}_{25} \mathrm{NO}_{2}$

Exact Mass: 347.1885

3ac was prepared according to general procedure 2.1 using $1 \mathbf{a}$ and $2 \mathrm{c}$ and was purified by silica gel column chromatography $(P E / E A=5 / 1)$ to obtain 3ac as yellow oil (50.7 mg, 73\% yield). ${ }^{1} \mathrm{H}$ NMR $\left(400 \mathrm{MHz}, \mathrm{CDCl}_{3}\right) \delta 7.34-7.27$ (m, $5 \mathrm{H}), 7.04-6.97(\mathrm{~m}, 2 \mathrm{H}), 6.49-6.44(\mathrm{~m}, 2 \mathrm{H}), 3.24(\mathrm{~d}, J=16.8 \mathrm{~Hz}, 1 \mathrm{H}), 2.89(\mathrm{~s}$, $6 \mathrm{H}), 2.65(\mathrm{~d}, J=16.9 \mathrm{~Hz}, 1 \mathrm{H}), 2.43-2.34(\mathrm{~m}, 1 \mathrm{H}), 2.15-1.98(\mathrm{~m}, 3 \mathrm{H}), 1.95-1.84$ (m, 1H), $1.20(\mathrm{~s}, 3 \mathrm{H}) ;{ }^{13} \mathrm{C}$ NMR $\left(101 \mathrm{MHz}, \mathrm{CDCl}_{3}\right) \delta 222.4,149.7,142.0,136.8$, 
136.4, 129.6, 129.1, 128.5, 127.1, 123.1, 111.4, 92.8, 56.1, 44.3, 40.1, 36.5, 28.7, 15.6; HRMS: (ESI) calcd for $\mathrm{C}_{23} \mathrm{H}_{26} \mathrm{NO}_{2}+[\mathrm{M}+\mathrm{H}]^{+} 348.1958$; found 348.1952 .

5-(4-fluorophenyl)-3a-hydroxy-6a-methyl-4-phenyl-3,3a,6,6a-tetrahydropental en-1(2H)-one (3ad)

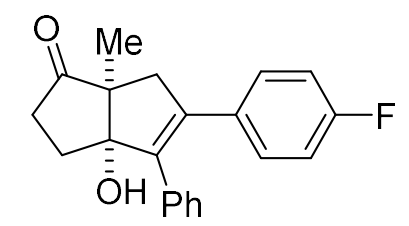

Chemical Formula: $\mathrm{C}_{21} \mathrm{H}_{19} \mathrm{FO}_{2}$

Exact Mass: 322.1369

3ad was prepared according to general procedure 2.1 using $\mathbf{1 a}$ and $\mathbf{2 d}$ and was purified by silica gel column chromatography $(P E / E A=7 / 1)$ to obtain 3ad as white solid (21.2 mg, 33\% yield). ${ }^{1} \mathrm{H}$ NMR $\left(400 \mathrm{MHz}, \mathrm{CDCl}_{3}\right) \delta 7.33-7.28$ (m, $3 \mathrm{H}), 7.25-7.21(\mathrm{~m}, 2 \mathrm{H}), 7.10-7.04(\mathrm{~m}, 2 \mathrm{H}), 6.87-6.80(\mathrm{~m}, 2 \mathrm{H}), 3.21(\mathrm{~d}, J=17.1$ $\mathrm{Hz}, 1 \mathrm{H}), 2.70(\mathrm{~d}, J=17.1 \mathrm{~Hz}, 1 \mathrm{H}), 2.48-2.39(\mathrm{~m}, 1 \mathrm{H}), 2.19-2.04(\mathrm{~m}, 2 \mathrm{H})$, 2.02-1.88 (m, 2H), $1.22(\mathrm{~s}, 3 \mathrm{H}) ;{ }^{13} \mathrm{C}$ NMR (101 MHz, $\left.\mathrm{CDCl}_{3}\right) \delta 221.7,162.1$ (d, $J=248.3 \mathrm{~Hz}$ ), 141.3, 140.0, 135.6, $131.6(\mathrm{~d}, J=3.2 \mathrm{~Hz}), 130.3(\mathrm{~d}, J=8.0 \mathrm{~Hz})$, 128.9, 128.7, 127.6, 115.0 (d, $J=21.3 \mathrm{~Hz}$ ), 92.7, 56.3, 44.7, 36.5, 29.1, 15.4; ${ }^{19} \mathrm{~F}$ NMR (376 MHz, CDCl$) \delta-113.1$; HRMS: (ESI) calcd for $\mathrm{C}_{21} \mathrm{H}_{19} \mathrm{FNaO}_{2}{ }^{+}$ $[\mathrm{M}+\mathrm{Na}]^{+} 345.1261$; found 345.1268 .

3a-hydroxy-6a-methyl-4-phenyl-5-(4-(4,4,5,5-tetramethyl-1,3,2-dioxaborolan-2 -yl)phenyl)-3,3a,6,6a-tetrahydropentalen-1(2H)-one (3ae)<smiles>CC1(C)OB(c2ccc(C3=C(c4ccccc4)C4(O)CCC(=O)C34C)cc2)OC1(C)C</smiles>

Chemical Formula: $\mathrm{C}_{27} \mathrm{H}_{31} \mathrm{BO}_{4}$

Exact Mass: 430.2315

3ae was prepared according to general procedure 2.1 using $1 \mathbf{a}$ and $2 \mathrm{e}$ and 
was purified by silica gel column chromatography $(P E / E A=5 / 1)$ to obtain 3ae as yellow oil (50.0 mg, $58 \%$ yield). ${ }^{1} \mathrm{H} \mathrm{NMR}\left(400 \mathrm{MHz}, \mathrm{CDCl}_{3}\right) \delta 7.60-7.55(\mathrm{~m}$, $2 \mathrm{H})$, 7.27-7.24 (m, 3H), 7.23-7.19 (m, 2H), 7.10-7.06 (m, 2H), $3.23(\mathrm{~d}, J=17.1$ $\mathrm{Hz}, 1 \mathrm{H}), 2.70(\mathrm{~d}, J=17.1 \mathrm{~Hz}, 1 \mathrm{H}), 2.47-2.37(\mathrm{~m}, 1 \mathrm{H}), 2.20-2.14(\mathrm{~m}, 1 \mathrm{H})$, 2.10-2.00 (m, 2H), 1.97-1.88 (m, 1H), $1.29(\mathrm{~s}, 12 \mathrm{H}), 1.21(\mathrm{~s}, 3 \mathrm{H}) ;{ }^{13} \mathrm{C}$ NMR $\left(101 \mathrm{MHz}, \mathrm{CDCl}_{3}\right) \delta 221.8,142.6,140.8,138.4,135.5,134.4,128.9,128.5$, 127.8, 127.5, 92.7, 83.8, 56.4, 44.7, 36.5, 29.0, 24.8, 15.5; HRMS: (ESI) calcd for $\mathrm{C}_{27} \mathrm{H}_{31} \mathrm{BNaO}_{4}{ }^{+}[\mathrm{M}+\mathrm{Na}]^{+} 453.2208$; found 453.2218 .

3a-hydroxy-6a-methyl-4-phenyl-5-(o-tolyl)-3,3a,6,6a-tetrahydropentalen-1(2H) -one (3af)

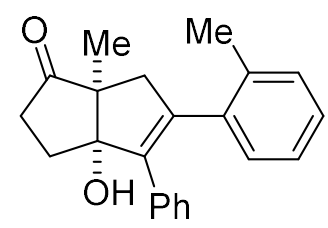

Chemical Formula: $\mathrm{C}_{22} \mathrm{H}_{22} \mathrm{O}_{2}$

Exact Mass: 318.1620

3af was prepared according to general procedure 2.1 using $\mathbf{1 a}$ and $\mathbf{2} \mathbf{f}$ and was purified by silica gel column chromatography $(\mathrm{PE} / \mathrm{EA}=7 / 1)$ to obtain 3af as yellow solid (21.0 mg, 33\% yield). ${ }^{1} \mathrm{H}$ NMR $\left(400 \mathrm{MHz}, \mathrm{CDCl}_{3}\right) \delta$ 7.23-7.20 (m, 2H), 7.18-7.14 (m, 3H), 7.12-7.05 (m, 3H), 6.99-6.96 (m, 1H), 3.07 (d, J = 17.4 $\mathrm{Hz}, 1 \mathrm{H}), 2.64(\mathrm{~d}, J=17.5 \mathrm{~Hz}, 1 \mathrm{H}), 2.53-2.42(\mathrm{~m}, 1 \mathrm{H}), 2.39-2.30(\mathrm{~m}, 1 \mathrm{H})$, 2.27-2.04 (m, 3H), $2.01(\mathrm{~s}, 3 \mathrm{H}), 1.22(\mathrm{~s}, 3 \mathrm{H}) ;{ }^{13} \mathrm{C} \mathrm{NMR}\left(101 \mathrm{MHz}, \mathrm{CDCl}_{3}\right) \delta$ $221.8,144.8,140.7,136.7,135.3,134.8,130.2,128.5,128.3,128.1,127.5$, 127.2, 125.8, 92.4, 57.1, 46.6, 36.7, 29.4, 19.6, 15.2; HRMS: (ESI) calcd for $\mathrm{C}_{22} \mathrm{H}_{22} \mathrm{NaO}_{2}{ }^{+}[\mathrm{M}+\mathrm{Na}]^{+} 341.1512$; found 341.1513 .

3a-hydroxy-6a-methyl-5-(naphthalen-2-yl)-4-phenyl-3,3a,6,6a-tetrahydropenta len-1(2H)-one (3ag) 


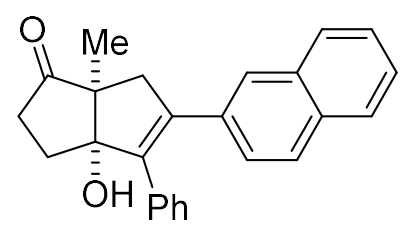

Chemical Formula: $\mathrm{C}_{25} \mathrm{H}_{22} \mathrm{O}_{2}$

Exact Mass: 354.1620

3ag was prepared according to general procedure 2.1 using $\mathbf{1 a}$ and $\mathbf{2 g}$ and was purified by silica gel column chromatography $(P E / E A=7 / 1)$ to obtain 3ag as yellow solid (61.6 mg, 87\% yield). ${ }^{1} \mathrm{H}$ NMR (400 $\left.\mathrm{MHz}, \mathrm{CDCl}_{3}\right) \delta 7.73-7.65$ $(\mathrm{m}, 3 \mathrm{H}), 7.53(\mathrm{~d}, J=8.6 \mathrm{~Hz}, 1 \mathrm{H}), 7.44-7.40(\mathrm{~m}, 2 \mathrm{H}), 7.31-7.27(\mathrm{~m}, 5 \mathrm{H}), 7.11(\mathrm{~d}$, $J=8.6 \mathrm{~Hz}, 1 \mathrm{H}), 3.40(\mathrm{~d}, J=17.1 \mathrm{~Hz}, 1 \mathrm{H}), 2.80(\mathrm{~d}, J=17.0 \mathrm{~Hz}, 1 \mathrm{H}), 2.51-2.41$ $(\mathrm{m}, 1 \mathrm{H}), 2.25-1.92(\mathrm{~m}, 4 \mathrm{H}), 1.26(\mathrm{~s}, 3 \mathrm{H}) ;{ }^{13} \mathrm{C}$ NMR (101 MHz, $\left.\mathrm{CDCl}_{3}\right) \delta 222.0$, $142.4,140.5,135.7,133.1,133.0,132.7,129.0,128.6,128.1,128.0,127.6$, $127.4,127.3,126.5,126.3,126.1,92.8,56.4$, 44.8, 36.5, 29.0, 15.5; HRMS: (ESI) calcd for $\mathrm{C}_{25} \mathrm{H}_{22} \mathrm{NaO}_{2}{ }^{+}[\mathrm{M}+\mathrm{Na}]^{+} 377.1512$; found 377.1509.

5-(dibenzo[b, d]furan-2-yl)-3a-hydroxy-6a-methyl-4-phenyl-3,3a,6,6a-tetrahydr opentalen-1(2H)-one (3ah)

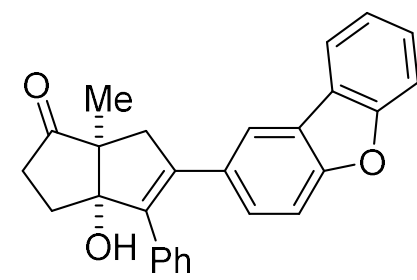

\section{Chemical Formula: $\mathrm{C}_{27} \mathrm{H}_{22} \mathrm{O}_{3}$ \\ Exact Mass: 394.1569}

3ah was prepared according to general procedure 2.1 using $\mathbf{1 a}$ and $\mathbf{2 h}$ and was purified by silica gel column chromatography $(P E / E A=5 / 1)$ to obtain 3ah as yellow oil (37.8 mg, 48\% yield). ${ }^{1} \mathrm{H}$ NMR (400 MHz, $\left.\mathrm{CDCl}_{3}\right) \delta$ 7.79-7.72 (m, 2H), $7.52(\mathrm{~d}, J=8.2 \mathrm{~Hz}, 1 \mathrm{H}), 7.46-7.40(\mathrm{~m}, 1 \mathrm{H}), 7.34-7.27(\mathrm{~m}, 7 \mathrm{H}), 7.18(\mathrm{~d}, J=$ $8.2 \mathrm{~Hz}, 1 \mathrm{H}), 3.38$ (d, J = 17.0 Hz, 1H), 2.82 (d, $J=17.0 \mathrm{~Hz}, 1 \mathrm{H}), 2.52-2.43(\mathrm{~m}$, $1 \mathrm{H}), 2.25-2.18(\mathrm{~m}, 1 \mathrm{H}), 2.16-2.04(\mathrm{~m}, 2 \mathrm{H}), 2.02-1.95(\mathrm{~m}, 1 \mathrm{H}), 1.27(\mathrm{~s}, 3 \mathrm{H}) ;{ }^{13} \mathrm{C}$ NMR $\left(101 \mathrm{MHz} \mathrm{CDCl}_{3}\right) \delta 222.0,156.5,155.5,142.5,139.7,135.8,129.1$, 
128.7, 128.1, 127.5, 127.3, 124.0, 123.9, 122.8, 120.8, 120.5, 111.7, 111.2, 92.8, 56.4, 45.2, 36.5, 29.0, 15.5; HRMS: (ESI) calcd for $\mathrm{C}_{27} \mathrm{H}_{22} \mathrm{NaO}_{3}{ }^{+}[\mathrm{M}+\mathrm{Na}]^{+}$ 417.1461; found 417.1475 .

5-(dibenzo[b, $d]$ thiophen-2-yl)-3a-hydroxy-6a-methyl-4-phenyl-3,3a,6,6a-tetrah ydropentalen-1(2H)-one (3ai)

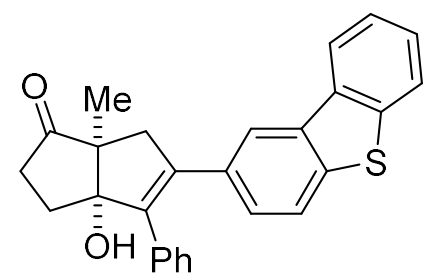

Chemical Formula: $\mathrm{C}_{27} \mathrm{H}_{22} \mathrm{O}_{2} \mathrm{~S}$

Exact Mass: 410.1341

3ai was prepared according to general procedure 2.1 using $\mathbf{1 a}$ and $\mathbf{2} \mathbf{i}$ and was purified by silica gel column chromatography $(P E / E A=5 / 1)$ to obtain 3ai as yellow solid (40.2 mg, 49\% yield). ${ }^{1} \mathrm{H}$ NMR $\left(400 \mathrm{MHz}, \mathrm{CDCl}_{3}\right) \delta 7.91$ (dd, $J=$ 1.9, $0.5 \mathrm{~Hz}, 1 \mathrm{H}), 7.87-7.83(\mathrm{~m}, 1 \mathrm{H}), 7.83-7.78(\mathrm{~m}, 1 \mathrm{H}), 7.59(\mathrm{~d}, J=8.4 \mathrm{~Hz}, 1 \mathrm{H})$, 7.45-7.37 (m, 2H), 7.35-7.29 (m, 5H), $7.18(\mathrm{~d}, J=8.4 \mathrm{~Hz}, 1 \mathrm{H}), 3.41(\mathrm{~d}, J=16.9$ $\mathrm{Hz}, 1 \mathrm{H}), 2.83(\mathrm{~d}, J=17.0 \mathrm{~Hz}, 1 \mathrm{H}), 2.54-2.44(\mathrm{~m}, 1 \mathrm{H}), 2.26-2.10(\mathrm{~m}, 2 \mathrm{H})$, 2.07-1.95 (m, 2H), $1.27(\mathrm{~s}, 3 \mathrm{H}) ;{ }^{13} \mathrm{C} \mathrm{NMR}\left(101 \mathrm{MHz}, \mathrm{CDCl}_{3}\right) \delta$ 221.9, 142.2, 140.2 , 139.6, 138.9, 135.9, 135.3, 135.3, 131.9, 129.0, 128.7, 127.6, 127.3, $126.8,124.5,122.8,122.3,121.6,121.4,92.8,56.4,44.9,36.6,29.1,15.5$; HRMS: (ESI) calcd for $\mathrm{C}_{27} \mathrm{H}_{22} \mathrm{NaO}_{2} \mathrm{~S}^{+}[\mathrm{M}+\mathrm{Na}]^{+} 433.1233$; found 433.1236 .

5-(benzo[b]thiophen-5-yl)-3a-hydroxy-6a-methyl-4-phenyl-3,3a,6,6a-tetrahydr opentalen $-1(2 H)$-one (3aj)

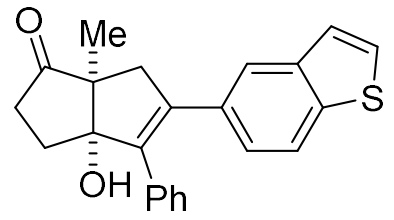

Chemical Formula: $\mathrm{C}_{23} \mathrm{H}_{20} \mathrm{O}_{2} \mathrm{~S}$

Exact Mass: 360.1184 
3aj was prepared according to general procedure $2.1 \mathbf{u s i n g} \mathbf{1 a}$ and $\mathbf{2} \mathbf{j}$ and was purified by silica gel column chromatography $(P E / E A=5 / 1)$ to obtain 3aj as white solid (49.7 mg, 69\% yield). ${ }^{1} \mathrm{H}$ NMR $\left(400 \mathrm{MHz}, \mathrm{CDCl}_{3}\right) \delta$ 7.65-7.57 (m, $2 \mathrm{H}), 7.38(\mathrm{~d}, J=5.5 \mathrm{~Hz}, 1 \mathrm{H}), 7.30-7.26(\mathrm{~m}, 5 \mathrm{H}), 7.19-7.17(\mathrm{~m}, 1 \mathrm{H}), 7.05-7.01$ (m, 1H), $3.33(\mathrm{~d}, J=17.0 \mathrm{~Hz}, 1 \mathrm{H}), 2.77(\mathrm{~d}, J=17.1 \mathrm{~Hz}, 1 \mathrm{H}), 2.51-2.40(\mathrm{~m}, 1 \mathrm{H})$, 2.22-1.91 (m, 4H), $\left.1.24(\mathrm{~s}, 3 \mathrm{H}) ;{ }^{13} \mathrm{C} \mathrm{NMR} \mathrm{(101} \mathrm{MHz,} \mathrm{CDCl}_{3}\right) \delta$ 222.0, 142.6, $139.9,139.4,139.1,135.7,131.9,129.0,128.6,127.5,126.8,125.0,124.0$, 123.7, 121.9, 92.8, 56.4, 45.0, 36.5, 29.0, 15.4; HRMS: (ESI) calcd for $\mathrm{C}_{23} \mathrm{H}_{20} \mathrm{NaO}_{2} \mathrm{~S}^{+}[\mathrm{M}+\mathrm{Na}]^{+}$383.1076; found 383.1082.

3a-hydroxy-6a-methyl-5-(6-morpholinopyridin-3-yl)-4-phenyl-3,3a,6,6a-tetrahy dropentalen-1(2H)-one (3ak)

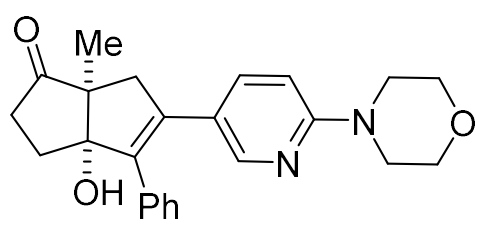

Chemical Formula: $\mathrm{C}_{24} \mathrm{H}_{26} \mathrm{~N}_{2} \mathrm{O}_{3}$

Exact Mass: 390.1943

3ak was prepared according to general procedure 2.1 using $\mathbf{1 a}$ and $\mathbf{2} \mathbf{k}$ and was purified by silica gel column chromatography $(P E / E A=4 / 1)$ to obtain 3ak as yellow solid (17.9 mg, $23 \%$ yield). ${ }^{1} \mathrm{H}$ NMR $\left(400 \mathrm{MHz}, \mathrm{CDCl}_{3}\right) \delta 8.00$ (dd, $J$ $=2.5,0.8 \mathrm{~Hz}, 1 \mathrm{H}), 7.35-7.29(\mathrm{~m}, 3 \mathrm{H}), 7.26-7.23(\mathrm{~m}, 2 \mathrm{H}), 7.17(\mathrm{dd}, J=8.9,2.5$ $\mathrm{Hz}, 1 \mathrm{H}), 6.37(\mathrm{dd}, J=9.0,0.8 \mathrm{~Hz}, 1 \mathrm{H}), 3.78-3.73(\mathrm{~m}, 4 \mathrm{H}), 3.46-3.42(\mathrm{~m}, 4 \mathrm{H})$, $3.20(\mathrm{~d}, J=16.9 \mathrm{~Hz}, 1 \mathrm{H}), 2.62(\mathrm{~d}, J=16.9 \mathrm{~Hz}, 1 \mathrm{H}), 2.46-2.38(\mathrm{~m}, 1 \mathrm{H})$, 2.14-2.00 (m, 3H), 1.92-1.87 (m, 1H), $\left.1.21(\mathrm{~s}, 3 \mathrm{H}) ;{ }^{13} \mathrm{C} \mathrm{NMR} \mathrm{(101} \mathrm{MHz,} \mathrm{CDCl}_{3}\right)$ $\delta 221.7,158.3,148.1,139.2,138.7,137.4,136.0,128.9,128.8,127.6,121.1$, 105.7, 92.5, 66.6, 56.2, 45.3, 43.8, 36.5, 29.0, 15.5; HRMS: (ESI) calcd for $\mathrm{C}_{24} \mathrm{H}_{27} \mathrm{~N}_{2} \mathrm{O}_{3}{ }^{+}[\mathrm{M}+\mathrm{H}]^{+} 391.2016$; found 391.2017.

3a-hydroxy-6a-methyl-5-(1-methyl-1H-indol-5-yl)-4-phenyl-3,3a,6,6a-tetrahydr 
opentalen-1(2H)-one (3al)

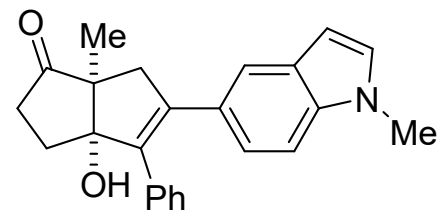

Chemical Formula: $\mathrm{C}_{24} \mathrm{H}_{23} \mathrm{NO}_{2}$

Exact Mass: 357.1729

3al was prepared according to general procedure 2.1 using $\mathbf{1 a}$ and $\mathbf{2 l}$ and was purified by silica gel column chromatography $(P E / E A=7 / 1)$ to obtain 3al as yellow solid (67.1 mg, 94\% yield). ${ }^{1} \mathrm{H}$ NMR (400 MHz, $\left.\mathrm{CDCl}_{3}\right) \delta 7.48$ (d, J = 1.6 $\mathrm{Hz}, 1 \mathrm{H}), 7.34-7.26(\mathrm{~m}, 5 \mathrm{H}), 7.05-6.93(\mathrm{~m}, 3 \mathrm{H}), 6.37(\mathrm{dd}, J=3.2,0.8 \mathrm{~Hz}, 1 \mathrm{H})$, 3.69 (s, 3H), 3.36 (d, J = 17.0 Hz, 1H), 2.75 (d, J = 17.1 Hz, 1H), 2.45-2.38 (m, $1 \mathrm{H}), 2.22-1.88(\mathrm{~m}, 4 \mathrm{H}), 1.24(\mathrm{~s}, 3 \mathrm{H}) ;{ }^{13} \mathrm{C} \mathrm{NMR}\left(101 \mathrm{MHz}, \mathrm{CDCl}_{3}\right) \delta 222.4$, $143.8,137.7,136.4,136.0,129.2$, 129.2, 128.4, 128.0, 127.0, 126.8, 122.7, 121.3, 108.6, 101.4, 92.8, 56.3, 45.2, 36.5, 32.7, 28.8, 15.5; HRMS: (ESI) calcd for $\mathrm{C}_{24} \mathrm{H}_{23} \mathrm{NNaO}_{2}{ }^{+}[\mathrm{M}+\mathrm{Na}]^{+} 380.1621$; found 380.1624 .

(3aS,6aS)-3a-hydroxy-6a-methyl-4-phenyl-5-((E)-styryl)-3,3a,6,6a-tetrahydrop entalen-1(2H)-one (3am)<smiles>CC12CC(/C=C/c3ccccc3)=C(c3ccccc3)[C@@]1(O)CCC2=O</smiles>

Chemical Formula: $\mathrm{C}_{23} \mathrm{H}_{22} \mathrm{O}_{2}$

Exact Mass: 330.1620

3am was prepared according to general procedure 2.1 using $1 \mathrm{a}$ and $2 \mathrm{~m}$ and was purified by silica gel column chromatography $(P E / E A=7 / 1)$ to obtain 3am as white solid (11.9 mg, 36\% yield). ${ }^{1} \mathrm{H}$ NMR (600 MHz, $\left.\mathrm{CDCl}_{3}\right) \delta 7.45-7.36(\mathrm{~m}$, 5H), 7.33-7.27 (m, 4H), 7.24-7.20 (m, 1H), $6.91(\mathrm{~d}, J=16.2 \mathrm{~Hz}, 1 \mathrm{H}), 6.67(\mathrm{~d}, J$ $=16.1 \mathrm{~Hz}, 1 \mathrm{H}), 3.14(\mathrm{~d}, J=16.5 \mathrm{~Hz}, 1 \mathrm{H}), 2.63(\mathrm{~d}, J=16.5 \mathrm{~Hz}, 1 \mathrm{H}), 2.44-2.37$ $(\mathrm{m}, 1 \mathrm{H}), 2.23-2.17(\mathrm{~m}, 1 \mathrm{H}), 2.05-1.94(\mathrm{~m}, 3 \mathrm{H}), 1.22(\mathrm{~s}, 3 \mathrm{H}) ;{ }^{13} \mathrm{C}$ NMR $(151$ $\left.\mathrm{MHz}_{2} \mathrm{CDCl}_{3}\right) \delta 221.6,142.5,141.0,136.8,134.9,133.8,129.2,128.6,128.5$, 
128.1, 127.8, 126.7, 123.1, 92.3, 56.3, 41.0, 36.5, 29.1, 15.6; HRMS: (ESI) calcd for $\mathrm{C}_{23} \mathrm{H}_{23} \mathrm{O}_{2}+[\mathrm{M}+\mathrm{H}]^{+} 331.1693$; found 331.1680.

3a-hydroxy-6a-methyl-5-phenyl-4-(p-tolyl)-3,3a,6,6a-tetrahydropentalen-1 $(2 H)$ -one (3ba)

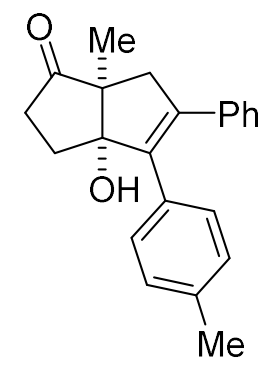

Chemical Formula: $\mathrm{C}_{22} \mathrm{H}_{22} \mathrm{O}_{2}$

Exact Mass: 318.1620

3ba was prepared according to general procedure 2.1 using $\mathbf{1 b}$ and $\mathbf{2 a}$ and was purified by silica gel column chromatography $(P E / E A=7 / 1)$ to obtain 3 ba as white solid (51.5 mg, 81\% yield). ${ }^{1} \mathrm{H}$ NMR $\left(400 \mathrm{MHz}, \mathrm{CDCl}_{3}\right) \delta$ 7.18-7.12 (m, 7H), 7.11-7.08 (m, 2H), $3.23(\mathrm{~d}, J=17.1 \mathrm{~Hz}, 1 \mathrm{H}), 2.70(\mathrm{~d}, J=17.0 \mathrm{~Hz}, 1 \mathrm{H})$, 2.45-2.37 (m, 1H), $2.34(\mathrm{~s}, 3 \mathrm{H}), 2.30-2.11(\mathrm{~m}, 2 \mathrm{H}), 2.10-2.00(\mathrm{~m}, 1 \mathrm{H})$, 1.97-1.87 (m, 1H), $1.21(\mathrm{~s}, 3 \mathrm{H}) ;{ }^{13} \mathrm{C}$ NMR $\left(101 \mathrm{MHz}, \mathrm{CDCl}_{3}\right) \delta 222.1,141.9$, 140.0, 137.1, 135.8, 132.6, 129.2, 128.8, 128.5, 128.0, 127.7, 92.7, 56.2, 44.7, 36.5, 29.0, 21.2, 15.5; HRMS: (ESI) calcd for $\mathrm{C}_{22} \mathrm{H}_{22} \mathrm{NaO}_{2}{ }^{+}[\mathrm{M}+\mathrm{Na}]^{+} 341.1512$; found 341.1502 .

3a-hydroxy-4-(4-methoxyphenyl)-6a-methyl-5-phenyl-3,3a,6,6a-tetrahydropen talen-1(2H)-one (3ca) 


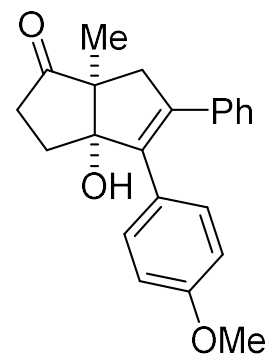

Chemical Formula: $\mathrm{C}_{22} \mathrm{H}_{22} \mathrm{O}_{3}$

Exact Mass: 334.1569

$3 \mathbf{c a}$ was prepared according to general procedure 2.1 using $\mathbf{1 c}$ and $\mathbf{2 a}$ and was purified by silica gel column chromatography $(P E / E A=5 / 1)$ to obtain 3ca as yellow solid (57.4 mg, 86\% yield). ${ }^{1} \mathrm{H}$ NMR (400 $\left.\mathrm{MHz}, \mathrm{CDCl}_{3}\right) \delta 7.20-7.17$ $(\mathrm{m}, 2 \mathrm{H}), 7.16-7.10(\mathrm{~m}, 5 \mathrm{H}), 6.84-6.79(\mathrm{~m}, 2 \mathrm{H}), 3.79(\mathrm{~s}, 3 \mathrm{H}), 3.22(\mathrm{~d}, J=17.0$ $\mathrm{Hz}, 1 \mathrm{H}), 2.68(\mathrm{~d}, J=17.1 \mathrm{~Hz}, 1 \mathrm{H}), 2.46-2.34(\mathrm{~m}, 1 \mathrm{H}), 2.26(\mathrm{~s}, 1 \mathrm{H}), 2.21-2.14$ (m, 1H), 2.08-1.98 (m, 1H), 1.97-1.87 (m, 1H), $1.20(\mathrm{~s}, 3 \mathrm{H}) ;{ }^{13} \mathrm{C}$ NMR $(101$ $\left.\mathrm{MHz}_{2} \mathrm{CDCl}_{3}\right) \delta 222.1,158.8,141.6,139.6,135.8,130.1,128.5,128.0,127.8$, 127.6, 113.9, 92.7, 56.2, 55.1, 44.7, 36.5, 28.9, 15.5; HRMS: (ESI) calcd for $\mathrm{C}_{22} \mathrm{H}_{22} \mathrm{NaO}_{3}{ }^{+}[\mathrm{M}+\mathrm{Na}]^{+} 357.1461$; found 357.1465.

4-(4-chlorophenyl)-3a-hydroxy-6a-methyl-5-phenyl-3,3a,6,6a-tetrahydropental en-1(2H)-one (3da)

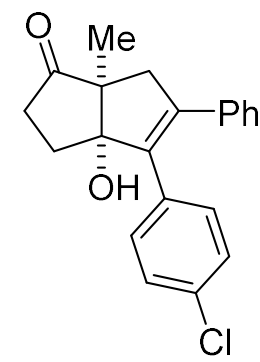

Chemical Formula: $\mathrm{C}_{21} \mathrm{H}_{19} \mathrm{ClO}_{2}$

Exact Mass: 338.1074

3da was prepared according to general procedure 2.1 using $\mathbf{1 d}$ and $\mathbf{2 a}$ and was purified by silica gel column chromatography $(P E / E A=5 / 1)$ to obtain 3da as yellow oil (49.3 mg, 73\% yield). ${ }^{1} \mathrm{H}$ NMR (400 MHz, $\left.\mathrm{CDCl}_{3}\right) \delta$ 7.26-7.19 (m, 4H), 7.19-7.15 (m, 3H), 7.12-7.07 (m, 2H), $3.25(\mathrm{~d}, J=17.3 \mathrm{~Hz}, 1 \mathrm{H}), 2.68(\mathrm{~d}, J$ 
$=17.2 \mathrm{~Hz}, 1 \mathrm{H}), 2.48-2.38(\mathrm{~m}, 1 \mathrm{H}), 2.19-2.08(\mathrm{~m}, 2 \mathrm{H}), 2.05-1.89(\mathrm{~m}, 2 \mathrm{H}), 1.21$ (s, 3H); ${ }^{13} \mathrm{C} \mathrm{NMR}\left(101 \mathrm{MHz}, \mathrm{CDCl}_{3}\right) \delta 221.7,143.4,138.7,135.3,134.1,133.3$, 130.3, 128.7, 128.6, 128.2, 128.0, 92.7, 56.4, 44.9, 36.4, 28.9, 15.4; HRMS: (ESI) calcd for $\mathrm{C}_{21} \mathrm{H}_{19} \mathrm{CINaO}_{2}{ }^{+}[\mathrm{M}+\mathrm{Na}]^{+} 361.0966$; found 361.0957 .

4-(4-fluorophenyl)-3a-hydroxy-6a-methyl-5-phenyl-3,3a,6,6a-tetrahydropental en-1(2H)-one (3ea)

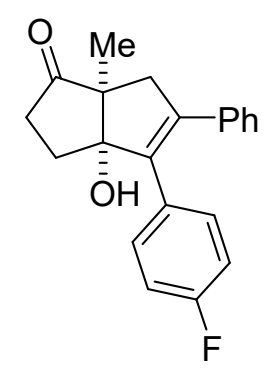

Chemical Formula: $\mathrm{C}_{21} \mathrm{H}_{19} \mathrm{FO}_{2}$

Exact Mass: 322.1369

3ea was prepared according to general procedure 2.1 using $\mathbf{1 e}$ and $\mathbf{2 a}$ and was purified by silica gel column chromatography $(P E / E A=5 / 1)$ to obtain 3ea as yellow solid (45.7 mg, $71 \%$ yield). ${ }^{1} \mathrm{H}$ NMR $\left(400 \mathrm{MHz}, \mathrm{CDCl}_{3}\right) \delta 7.25-7.21$ $(\mathrm{m}, 2 \mathrm{H}), 7.19-7.14(\mathrm{~m}, 3 \mathrm{H}), 7.11-7.07(\mathrm{~m}, 2 \mathrm{H}), 7.01-6.94(\mathrm{~m}, 2 \mathrm{H}), 3.24(\mathrm{~d}, J=$ $17.2 \mathrm{~Hz}, 1 \mathrm{H}), 2.69(\mathrm{~d}, J=17.2 \mathrm{~Hz}, 1 \mathrm{H}), 2.49-2.39(\mathrm{~m}, 1 \mathrm{H}), 2.18-2.10(\mathrm{~m}, 2 \mathrm{H})$, 2.08-1.99 (m, 1H), 1.98-1.89 (m, 1H), $\left.1.21(\mathrm{~s}, 3 \mathrm{H}) ;{ }^{13} \mathrm{C} \mathrm{NMR} \mathrm{(101} \mathrm{MHz,} \mathrm{CDCl}_{3}\right)$ $\delta$ 221.7, 162.2 (d, $J=246.9 \mathrm{~Hz}), 142.9,138.9,135.4,131.5(\mathrm{~d}, J=3.6 \mathrm{~Hz})$, $130.7(\mathrm{~d}, J=7.9 \mathrm{~Hz}), 128.5,128.1,127.9,115.5(\mathrm{~d}, J=21.2 \mathrm{~Hz}), 92.7,56.3$, 44.8, 36.4, 28.9, 15.4; ${ }^{19} \mathrm{FNMR}\left(376 \mathrm{MHz}, \mathrm{CDCl}_{3}\right.$ ) $\delta$-114.2; HRMS: (ESI) calcd for $\mathrm{C}_{21} \mathrm{H}_{19} \mathrm{FNaO}{ }_{2}{ }^{+}[\mathrm{M}+\mathrm{Na}]^{+} 345.1261$; found 345.1252.

3a-hydroxy-6a-methyl-5-phenyl-4-(4-(trifluoromethyl)phenyl)-3,3a,6,6a-tetrahy dropentalen-1(2H)-one (3fa) 


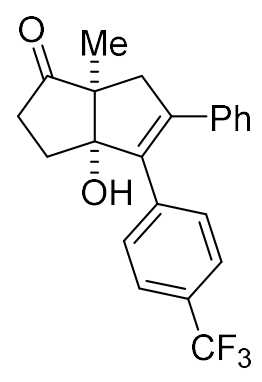

Chemical Formula: $\mathrm{C}_{22} \mathrm{H}_{19} \mathrm{~F}_{3} \mathrm{O}_{2}$

Exact Mass: 372.1337

3fa was prepared according to general procedure 2.1 using $1 \mathbf{f}$ and $\mathbf{2 a}$ and was purified by silica gel column chromatography $(\mathrm{PE} / \mathrm{EA}=5 / 1)$ to obtain $3 \mathrm{fa}$ as yellow solid (59.5 mg, 80\% yield). ${ }^{1} \mathrm{H}$ NMR $\left(400 \mathrm{MHz}, \mathrm{CDCl}_{3}\right) \delta 7.52(\mathrm{~d}, J=8.1$ $\mathrm{Hz}, 2 \mathrm{H}), 7.40(\mathrm{~d}, \mathrm{~J}=8.0 \mathrm{~Hz}, 2 \mathrm{H}), 7.22-7.14(\mathrm{~m}, 3 \mathrm{H}), 7.10-7.04(\mathrm{~m}, 2 \mathrm{H}), 3.28(\mathrm{~d}$, $J=17.4 \mathrm{~Hz}, 1 \mathrm{H}), 2.72(\mathrm{~d}, J=17.4 \mathrm{~Hz}, 1 \mathrm{H}), 2.53-2.27(\mathrm{~m}, 2 \mathrm{H}), 2.14-1.93(\mathrm{~m}$, 3H), $1.22(\mathrm{~s}, 3 \mathrm{H}) ;{ }^{13} \mathrm{C} \mathrm{NMR}\left(101 \mathrm{MHz}, \mathrm{CDCl}_{3}\right) \delta 221.5,144.5,139.6,138.6$, 135.1, 129.4 (q, $J=32.5 \mathrm{~Hz}), 129.3,128.6,128.3,128.2,125.4$ (q, $J=3.8 \mathrm{~Hz}$ ), $\delta 124.1$ (q, $J=273.7 \mathrm{~Hz}), 92.8,56.5,45.1,36.4,29.0,15.3 ;{ }^{19} \mathrm{~F}$ NMR (376 $\left.\mathrm{MHz}, \mathrm{CDCl}_{3}\right) \delta$-62.4; HRMS: (ESI) calcd for $\mathrm{C}_{22} \mathrm{H}_{19} \mathrm{~F}_{3} \mathrm{NaO}_{2}{ }^{+}[\mathrm{M}+\mathrm{Na}]^{+}$395.1229; found 395.1222 .

ethyl-4-(6a-hydroxy-3a-methyl-4-oxo-2-phenyl-3,3a,4,5,6,6a-hexahydropental en-1-yl)benzoate (3ga)<smiles>CCOC(=O)c1ccc(C2=C(c3ccccc3)CC3(C)C(=O)CCC23O)cc1</smiles>

Chemical Formula: $\mathrm{C}_{24} \mathrm{H}_{24} \mathrm{O}_{4}$

Exact Mass: 376.1675

3ga was prepared according to general procedure 2.1 using $\mathbf{1 g}$ and $\mathbf{2 a}$ and was purified by silica gel column chromatography $(\mathrm{PE} / \mathrm{EA}=5 / 1)$ to obtain 3ga as yellow oil $\left(57.1 \mathrm{mg}, 76 \%\right.$ yield). ${ }^{1} \mathrm{H} \mathrm{NMR}\left(400 \mathrm{MHz}, \mathrm{CDCl}_{3}\right) \delta 7.98-7.89(\mathrm{~m}$, 
2H), 7.37-7.30 (m, 2H), 7.19-7.11 (m, 3H), 7.10-7.04 (m, 2H), $4.34(\mathrm{q}, J=7.0$ $\mathrm{Hz}, 2 \mathrm{H}), 3.27(\mathrm{~d}, J=17.3 \mathrm{~Hz}, 1 \mathrm{H}), 2.71(\mathrm{~d}, J=17.3 \mathrm{~Hz}, 1 \mathrm{H}), 2.55-2.36(\mathrm{~m}, 2 \mathrm{H})$, 2.12-1.90 (m, 3H), 1.36 (t, $J=7.1 \mathrm{~Hz}, 3 \mathrm{H}), 1.21(\mathrm{~s}, 3 \mathrm{H}) ;{ }^{13} \mathrm{C}$ NMR $(101 \mathrm{MHz}$, $\left.\mathrm{CDCl}_{3}\right) \delta 221.6,166.5,144.2,140.7,139.1,135.2,129.7,129.3,129.0,128.6$, 128.2, 128.1, 92.7, 61.0, 56.5, 45.0, 36.4, 28.9, 15.3, 14.3; HRMS: (ESI) calcd for $\mathrm{C}_{24} \mathrm{H}_{24} \mathrm{NaO}_{4}{ }^{+}[\mathrm{M}+\mathrm{Na}]^{+} 399.1567$; found 399.1559.

3a-hydroxy-6a-methyl-5-phenyl-4-(4-(4,4,5,5-tetramethyl-1,3,2-dioxaborolan-2 -yl)phenyl)-3,3a,6,6a-tetrahydropentalen-1(2H)-one (3ha)

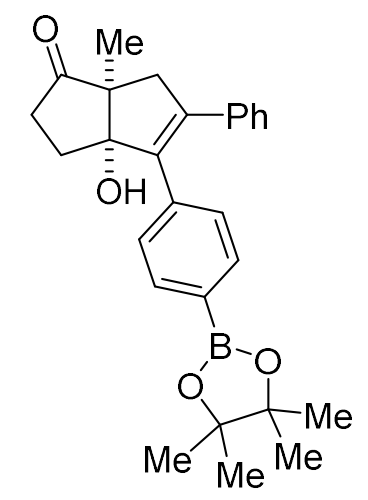

Chemical Formula: $\mathrm{C}_{27} \mathrm{H}_{31} \mathrm{BO}_{4}$

Exact Mass: 430.2315

3ha was prepared according to general procedure 2.1 using $1 \mathrm{~h}$ and $2 \mathrm{a}$ and was purified by silica gel column chromatography $(\mathrm{PE} / \mathrm{EA}=7 / 1)$ to obtain 3ha as white solid (57.6 mg, 67\% yield). ${ }^{1} \mathrm{H}$ NMR $\left(400 \mathrm{MHz}, \mathrm{CDCl}_{3}\right) \delta$ 7.77-7.70 (m, $2 \mathrm{H}), 7.27(\mathrm{~s}, 1 \mathrm{H}), 7.25(\mathrm{~s}, 1 \mathrm{H}), 7.16-7.08(\mathrm{~m}, 5 \mathrm{H}), 3.25(\mathrm{~d}, J=17.1 \mathrm{~Hz}, 1 \mathrm{H})$, $2.70(\mathrm{~d}, J=17.2 \mathrm{~Hz}, 1 \mathrm{H}), 2.45-2.22(\mathrm{~m}, 2 \mathrm{H}), 2.15-1.88(\mathrm{~m}, 3 \mathrm{H}), 1.34(\mathrm{~s}, 12 \mathrm{H})$, 1.21 (s, 3H); ${ }^{13} \mathrm{C} \mathrm{NMR}\left(101 \mathrm{MHz}, \mathrm{CDCl}_{3}\right) \delta 221.9,142.9,140.0,138.8,135.5$, 134.9, 128.6, 128.3, 128.1, 127.8, 92.7, 83.8, 56.4, 44.8, 36.5, 29.0, 24.9, 24.8, 15.4; HRMS: (ESI) calcd for $\mathrm{C}_{27} \mathrm{H}_{31} \mathrm{BNaO}_{4}^{+}[\mathrm{M}+\mathrm{Na}]^{+} 453.2208$; found 453.2201 .

3-(6a-hydroxy-3a-methyl-4-oxo-2-phenyl-3,3a,4,5,6,6a-hexahydropentalen-1yl)benzonitrile (3ia) 


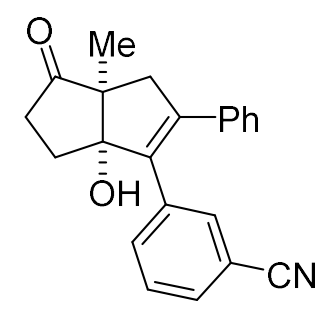

Chemical Formula: $\mathrm{C}_{22} \mathrm{H}_{19} \mathrm{NO}_{2}$

Exact Mass: 329.1416

3ia was prepared according to general procedure 2.1 using $1 \mathbf{i}$ and $\mathbf{2 a}$ and was purified by silica gel column chromatography $(P E / E A=5 / 1)$ to obtain 3ia as white solid (55.6 mg, 83\% yield). ${ }^{1} \mathrm{H}$ NMR $\left(400 \mathrm{MHz}, \mathrm{CDCl}_{3}\right) \delta$ 7.69-7.62 (m, $1 \mathrm{H})$, 7.53-7.48 $(\mathrm{m}, 1 \mathrm{H}), 7.46-7.42(\mathrm{~m}, 1 \mathrm{H}), 7.36-7.30(\mathrm{~m}, 1 \mathrm{H}), 7.21-7.13(\mathrm{~m}$, 3H), 7.06-7.01 (m, 2H), $3.25(\mathrm{~d}, J=17.4 \mathrm{~Hz}, 1 \mathrm{H}), 2.70(\mathrm{~d}, J=17.4 \mathrm{~Hz}, 1 \mathrm{H})$, $2.65(\mathrm{~s}, 1 \mathrm{H}), 2.50-2.39(\mathrm{~m}, 1 \mathrm{H}), 2.08-1.93(\mathrm{~m}, 3 \mathrm{H}), 1.21(\mathrm{~s}, 3 \mathrm{H}) ;{ }^{13} \mathrm{C}$ NMR $(101$ $\left.\mathrm{MHz}, \mathrm{CDCl}_{3}\right) \delta 221.4,144.9,137.6,137.2,134.9,133.7,132.3,130.9,129.2$, $128.5,128.3,128.3,118.6,112.4,92.6,56.4,45.1$, 36.3, 29.0, 15.3; HRMS: (ESI) calcd for $\left.\mathrm{C}_{22} \mathrm{H}_{19} \mathrm{NNaO}_{2}+\mathrm{M}+\mathrm{Na}\right]^{+} 352.1308$; found 352.1313 .

3a-hydroxy-6a-methyl-4-(naphthalen-2-yl)-5-phenyl-3,3a,6,6a-tetrahydropenta len-1(2H)-one (3ja)

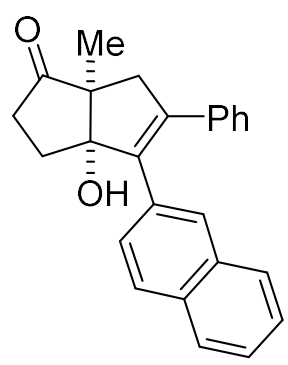

Chemical Formula: $\mathrm{C}_{25} \mathrm{H}_{22} \mathrm{O}_{2}$

Exact Mass: 354.1620

$\mathbf{3} \mathbf{j a}$ was prepared according to general procedure $2.1 \mathbf{u s i n g} \mathbf{1} \mathbf{j}$ and $\mathbf{2} \mathbf{a}$ and was purified by silica gel column chromatography $(P E / E A=10 / 1 ~ 5 / 1)$ to obtain 3ja as yellow solid (49.6 mg, $70 \%$ yield). ${ }^{1} \mathrm{H} \mathrm{NMR}\left(400 \mathrm{MHz}, \mathrm{CDCl}_{3}\right) \delta 7.89(\mathrm{~s}, 1 \mathrm{H})$, 7.83-7.72 (m, 3H), 7.50-7.44 (m, 2H), 7.31-7.28 (m, 1H), 7.18-7.10 (m, 5H), $3.34(\mathrm{~d}, J=17.1 \mathrm{~Hz}, 1 \mathrm{H}), 2.77(\mathrm{~d}, J=17.1 \mathrm{~Hz}, 1 \mathrm{H}), 2.51-2.39(\mathrm{~m}, 2 \mathrm{H})$, 2.24-1.93 (m, 3H), $1.27(\mathrm{~s}, 3 \mathrm{H}) ;{ }^{13} \mathrm{C}$ NMR $\left(101 \mathrm{MHz}, \mathrm{CDCl}_{3}\right) \delta 222.0,142.8$, 
$139.9,135.5,133.4,133.3,132.6,128.6,128.1,128.0,127.9,127.6,127.5$, 127.4, 126.0, 126.0, 92.9, 56.4, 44.8, 36.5, 29.0, 15.5; HRMS: (ESI) calcd for $\mathrm{C}_{25} \mathrm{H}_{22} \mathrm{NaO}_{2}{ }^{+}[\mathrm{M}+\mathrm{Na}]^{+} 377.1512$; found 377.1516 .

4-(benzo[d][1,3]dioxol-5-yl)-3a-hydroxy-6a-methyl-5-phenyl-3,3a,6,6a-tetrahyd ropentalen-1(2H)-one (3ka)

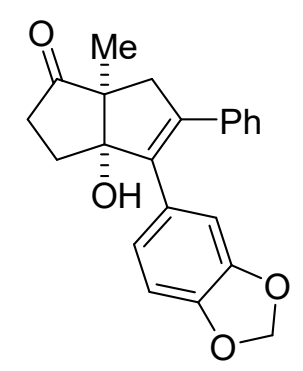

Chemical Formula: $\mathrm{C}_{22} \mathrm{H}_{20} \mathrm{O}_{4}$

Exact Mass: 348.1362

3ka was prepared according to general procedure $2.1 \mathrm{using} \mathbf{1 k}$ and $\mathbf{2 a}$ and was purified by silica gel column chromatography $(\mathrm{PE} / \mathrm{EA}=5 / 1)$ to obtain 3ka as yellow solid (54.2 mg, $78 \%$ yield). ${ }^{1} \mathrm{H}$ NMR $\left(400 \mathrm{MHz}, \mathrm{CDCl}_{3}\right) \delta 7.18-7.15$ (m, 3H), 7.15-7.11 (m, 2H), 6.80-6.76 (m, 1H), 6.74-6.68 (m, 2H), $5.94(\mathrm{~s}, 2 \mathrm{H})$, $3.21(\mathrm{~d}, J=17.1 \mathrm{~Hz}, 1 \mathrm{H}), 2.67(\mathrm{~d}, J=17.1 \mathrm{~Hz}, 1 \mathrm{H}), 2.48-2.37(\mathrm{~m}, 1 \mathrm{H})$, 2.25-2.12 (m, 2H), 2.08-1.98 (m, 1H), 1.96-1.87 (m, 1H), $1.20(\mathrm{~s}, 3 \mathrm{H}) ;{ }^{13} \mathrm{C}$ NMR $\left(101 \mathrm{MHz}, \mathrm{CDCl}_{3}\right) \delta 221.9,147.6,146.9,142.1,139.6,135.6,129.3$, 128.5, 128.0, 127.8, 122.6, 109.3, 108.5, 100.9, 92.6, 56.2, 44.6, 36.5, 29.0, 15.4; HRMS: (ESI) calcd for $\mathrm{C}_{22} \mathrm{H}_{20} \mathrm{NaO}_{4}{ }^{+}[\mathrm{M}+\mathrm{Na}]^{+} 371.1254$; found 371.1260 .

4-(2,3-dihydrobenzo[b][1,4]dioxin-6-yl)-3a-hydroxy-6a-methyl-5-phenyl-3,3a,6, 6a-tetrahydropentalen-1(2H)-one (3la) 


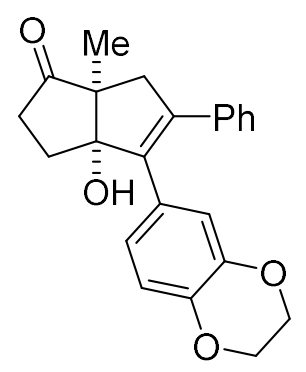

Chemical Formula: $\mathrm{C}_{23} \mathrm{H}_{22} \mathrm{O}_{4}$

Exact Mass: 362.1518

3la was prepared according to general procedure 2.1 using $1 \mathbf{l}$ and $2 \mathbf{a}$ and was purified by silica gel column chromatography $(P E / E A=5 / 1)$ to obtain 3la as white solid (56.5 mg, 78\% yield). ${ }^{1} \mathrm{H}$ NMR (400 MHz, $\left.\mathrm{CDCl}_{3}\right) \delta 7.18-7.11(\mathrm{~m}$, $5 \mathrm{H}), 6.85-6.82(\mathrm{~m}, 1 \mathrm{H}), 6.77-6.73(\mathrm{~m}, 1 \mathrm{H}), 6.67-6.63(\mathrm{~m}, 1 \mathrm{H}), 4.27-4.20(\mathrm{~m}$, $4 \mathrm{H}), 3.19(\mathrm{~d}, J=17.0 \mathrm{~Hz}, 1 \mathrm{H}), 2.67(\mathrm{~d}, J=17.0 \mathrm{~Hz}, 1 \mathrm{H}), 2.46-2.37(\mathrm{~m}, 1 \mathrm{H})$, 2.24-2.14 (m, 2H), 2.09-1.99 (m, $1 \mathrm{H}), 1.97-1.86(\mathrm{~m}, 1 \mathrm{H}), 1.19(\mathrm{~s}, 3 \mathrm{H}) ;{ }^{13} \mathrm{C}$ NMR (101 MHz, $\left.\mathrm{CDCl}_{3}\right) \delta 222.1,143.4,143.0,141.8,139.4,135.7,128.8$, $128.5,128.0,127.7,122.4,117.5,117.3,92.6,64.3,64.2$, 56.2, 44.7, 36.5, 29.0, 15.5; HRMS: (ESI) calcd for $\mathrm{C}_{23} \mathrm{H}_{22} \mathrm{NaO}_{4}^{+}[\mathrm{M}+\mathrm{Na}]^{+} 385.1410$; found 385.1403.

3a-hydroxy-6a-methyl-4-(1-methyl-1H-indol-4-yl)-5-phenyl-3,3a,6,6a-tetrahydr opentalen-1(2H)-one (3ma)

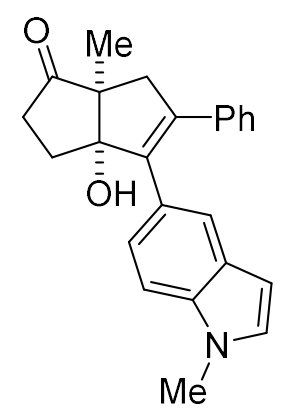

Chemical Formula: $\mathrm{C}_{24} \mathrm{H}_{23} \mathrm{NO}_{2}$

Exact Mass: 357.1729

3ma was prepared according to general procedure 2.1 using $1 \mathrm{~m}$ and $2 \mathrm{a}$ and was purified by silica gel column chromatography $(P E / E A=10 / 1 \sim 5 / 1)$ to obtain 3ma as white solid (55.7 mg, 78\% yield). ${ }^{1} \mathrm{H}$ NMR (400 $\left.\mathrm{MHz}, \mathrm{CDCl}_{3}\right) \delta$ 
7.62-7.60 (m, 1H), 7.25-7.22 (m, 1H), 7.18-7.14 (m, 2H), 7.13-7.09 (m, 3H), 7.06-7.03 (m, 2H), 6.46-6.44 (m, 1H), $3.78(\mathrm{~s}, 3 \mathrm{H}), 3.27(\mathrm{~d}, J=16.8 \mathrm{~Hz}, 1 \mathrm{H})$, $2.75(\mathrm{~d}, J=16.9 \mathrm{~Hz}, 1 \mathrm{H}), 2.48-2.35(\mathrm{~m}, 1 \mathrm{H}), 2.25-2.18(\mathrm{~m}, 2 \mathrm{H}), 2.16-2.05(\mathrm{~m}$, $1 \mathrm{H}), 1.97-1.87(\mathrm{~m}, 1 \mathrm{H}), 1.25(\mathrm{~s}, 3 \mathrm{H}) ;{ }^{13} \mathrm{C}$ NMR $\left(101 \mathrm{MHz}, \mathrm{CDCl}_{3}\right) \delta 222.3$, $141.2,140.8,136.1,136.1,129.1,128.6,128.6,127.9,127.4,126.6,123.1$, 121.0, 109.4, 101.2, 92.8, 56.2, 44.6, 36.6, 32.8, 29.1, 15.6; HRMS: (ESI) calcd for $\mathrm{C}_{24} \mathrm{H}_{23} \mathrm{NNaO}_{2}+[\mathrm{M}+\mathrm{Na}]^{+} 380.1621$; found 380.1621 .

4-(dibenzo[b, d] thiophen-2-yl)-3a-hydroxy-6a-methyl-5-phenyl-3,3a,6,6a-tetrah ydropentalen-1(2H)-one (3na)

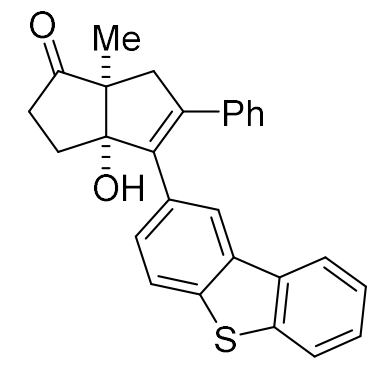

Chemical Formula: $\mathrm{C}_{27} \mathrm{H}_{22} \mathrm{O}_{2} \mathrm{~S}$

Exact Mass: 410.1341

3na was prepared according to general procedure 2.1 using $\mathbf{1 n}$ and $\mathbf{2 a}$ and was purified by silica gel column chromatography $(P E / E A=5 / 1)$ to obtain 3na as white solid (71.3 mg, 87\% yield). ${ }^{1} \mathrm{H}$ NMR $\left(400 \mathrm{MHz}, \mathrm{CDCl}_{3}\right) \delta 8.17-8.13(\mathrm{~m}$, $1 \mathrm{H})$, 8.06-8.00 (m, 1H), 7.88-7.81 (m, 1H), 7.77-7.72 (m, 1H), 7.48-7.39 (m, 2H), 7.33-7.28 (m, 1H), 7.19-7.09 (m, 5H), $3.34(\mathrm{~d}, J=17.1 \mathrm{~Hz}, 1 \mathrm{H}), 2.77(\mathrm{~d}, J$ $=17.2 \mathrm{~Hz}, 1 \mathrm{H}), 2.51-2.41(\mathrm{~m}, 1 \mathrm{H}), 2.36(\mathrm{~s}, 1 \mathrm{H}), 2.25-2.18(\mathrm{~m}, 1 \mathrm{H}), 2.15-2.04$ $(\mathrm{m}, 1 \mathrm{H}), 2.02-1.92(\mathrm{~m}, 1 \mathrm{H}), 1.27(\mathrm{~s}, 3 \mathrm{H}) ;{ }^{13} \mathrm{C} \mathrm{NMR}\left(101 \mathrm{MHz}, \mathrm{CDCl}_{3}\right) \delta$ 221.9, 142.8, 139.7, 139.6, 138.5, 135.8, 135.5, 135.3, 132.0, 128.6, 128.1, 127.9, 127.9, 126.8, 124.4, 122.9, 122.8, 121.6, 92.9, 56.4, 44.8, 36.5, 29.0, 15.5; HRMS: (ESI) calcd for $\mathrm{C}_{27} \mathrm{H}_{22} \mathrm{NaO}_{2} \mathrm{~S}^{+}[\mathrm{M}+\mathrm{Na}]^{+} 433.1233$; found 433.1239 .

4-(dibenzo[b, d]furan-2-yl)-3a-hydroxy-6a-methyl-5-phenyl-3,3a,6,6a-tetrahydr opentalen-1(2H)-one (3oa) 


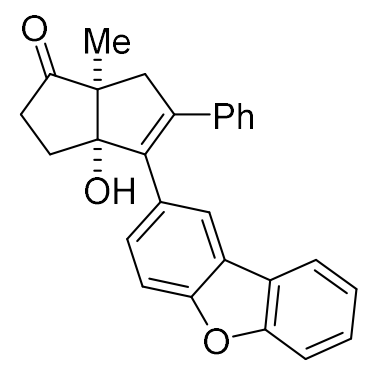

Chemical Formula: $\mathrm{C}_{27} \mathrm{H}_{22} \mathrm{O}_{3}$

Exact Mass: 394.1569

3oa was prepared according to general procedure 2.1 using 10 and 2 a and was purified by silica gel column chromatography $(P E / E A=5 / 1)$ to obtain 3oa as white solid $\left(67.0 \mathrm{mg}, 85 \%\right.$ yield). ${ }^{1} \mathrm{H}$ NMR $\left(400 \mathrm{MHz}, \mathrm{CDCl}_{3}\right) \delta 7.94(\mathrm{~d}, \mathrm{~J}=$ $1.8 \mathrm{~Hz}, 1 \mathrm{H}), 7.90-7.84(\mathrm{~m}, 1 \mathrm{H}), 7.60-7.54(\mathrm{~m}, 1 \mathrm{H}), 7.50-7.42(\mathrm{~m}, 2 \mathrm{H})$, 7.34-7.27 (m, 2H), 7.17-7.09 (m, 5H), $3.32(\mathrm{~d}, J=17.1 \mathrm{~Hz}, 1 \mathrm{H}), 2.76(\mathrm{~d}, J=$ $17.1 \mathrm{~Hz}, 1 \mathrm{H}), 2.52-2.29(\mathrm{~m}, 2 \mathrm{H}), 2.21-1.93(\mathrm{~m}, 3 \mathrm{H}), 1.27(\mathrm{~s}, 3 \mathrm{H}) ;{ }^{13} \mathrm{C}$ NMR $\left(101 \mathrm{MHz}, \mathrm{CDCl}_{3}\right) \delta 221.9,156.4,155.5,142.5,139.9,135.5,130.4,128.6$, $128.4,128.1,127.8,127.3,124.5,124.0,122.7,120.8,120.7,111.8,111.7$, 92.8, 56.3, 44.7, 36.5, 29.0, 15.5; HRMS: (ESI) calcd for $\mathrm{C}_{27} \mathrm{H}_{22} \mathrm{NaO}_{3}{ }^{+}[\mathrm{M}+\mathrm{Na}]^{+}$ 417.1461; found 417.1469 .

3a-hydroxy-4-(6-methoxypyridin-3-yl)-6a-methyl-5-phenyl-3,3a,6,6a-tetrahydr opentalen-1(2H)-one (3pa)

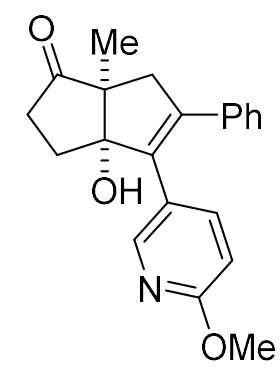

Chemical Formula: $\mathrm{C}_{21} \mathrm{H}_{21} \mathrm{NO}_{3}$

Exact Mass: 335.1521

$3 \mathbf{p a}$ was prepared according to general procedure 2.1 using $1 \mathbf{p}$ and $\mathbf{2 a}$ and was purified by silica gel column chromatography $(\mathrm{PE} / \mathrm{EA}=5 / 1)$ to obtain 3 pa as white solid (54.3 mg, 81\% yield). ${ }^{1} \mathrm{H}$ NMR $\left(400 \mathrm{MHz}, \mathrm{CDCl}_{3}\right) \delta 8.09-8.00$ (m, 
1H), 7.51-7.43 (m, 1H), 7.20-7.13 (m, 3H), 7.11-7.04 (m, 2H), 6.74-6.60 (m, $1 \mathrm{H}), 3.89(\mathrm{~s}, 3 \mathrm{H}), 3.20(\mathrm{~d}, J=17.2 \mathrm{~Hz}, 1 \mathrm{H}), 2.67(\mathrm{~d}, J=17.2 \mathrm{~Hz}, 1 \mathrm{H})$, 2.56-1.88 (m, 5H), $1.18(\mathrm{~s}, 3 \mathrm{H}) ;{ }^{13} \mathrm{C}$ NMR $\left(101 \mathrm{MHz}, \mathrm{CDCl}_{3}\right) \delta 221.8,163.2$, 146.7, 143.5, 139.4, 136.4, 135.4, 128.5, 128.2, 128.0, 124.4, 110.5, 92.6, 56.3, 53.4, 45.1, 36.4, 29.0, 15.4; HRMS: (ESI) calcd for $\mathrm{C}_{21} \mathrm{H}_{21} \mathrm{NO}_{3}{ }^{+}[\mathrm{M}+\mathrm{H}]^{+}$ 336.1594 ; found 336.1592 .

(8R,9S, 13S, 14S)-3-(6a-hydroxy-3a-methyl-4-oxo-2-phenyl-3,3a,4,5,6,6a-hexa hydropetalen-1-yl)-13-methyl-6,7,8,9,11,12,13,14,15,16-decahydro-17H-

cyclopenta[a]phenanthren-17-one (3qa)

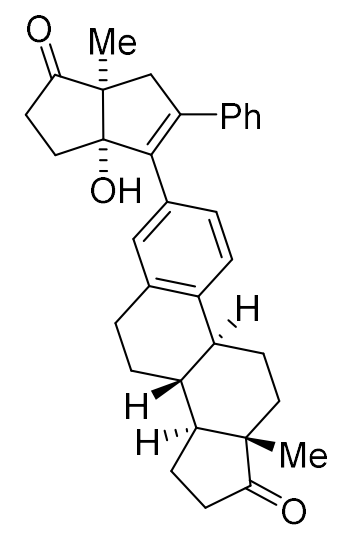

Chemical Formula: $\mathrm{C}_{33} \mathrm{H}_{36} \mathrm{O}_{3}$

Exact Mass: 480.2664

3qa was prepared according to general procedure 2.1 using $\mathbf{1 q}$ and $\mathbf{2 a}$ and was purified by silica gel column chromatography $(P E / E A=7 / 1)$ to obtain $3 q a$ as white solid $(75.8 \mathrm{mg}, 79 \%$ yield, $1: 1 \mathrm{~d} . \mathrm{r}) .{ }^{1} \mathrm{H}$ NMR $\left(400 \mathrm{MHz}, \mathrm{CDCl}_{3}\right) \delta$ 7.18-7.15 (m, 1H), 7.15-7.11 (m, 5H), 7.03-6.95 (m, 2H), 3.20 (dd, J = 17.0, 1.9 $\mathrm{Hz}, 1 \mathrm{H}), 2.84-2.76(\mathrm{~m}, 2 \mathrm{H}), 2.70(\mathrm{~d}, J=17.0 \mathrm{~Hz}, 1 \mathrm{H}), 2.52-2.35(\mathrm{~m}, 4 \mathrm{H})$, 2.32-2.25 (m, $1 \mathrm{H}), 2.24-2.12(\mathrm{~m}, 2 \mathrm{H}), 2.10-2.00(\mathrm{~m}, 3 \mathrm{H}), 1.98-1.91(\mathrm{~m}, 2 \mathrm{H})$, 1.64-1.56 (m, 2H), 1.53-1.41 (m, 4H), $1.20(\mathrm{~s}, 3 \mathrm{H}), 0.91(\mathrm{~s}, 3 \mathrm{H}) ;{ }^{13} \mathrm{C}$ NMR $(101$ $\left.\mathrm{MHz}, \mathrm{CDCl}_{3}\right) \delta 222.1,220.9,141.7,140.0,139.9,138.8,136.4,135.9,129.2$, $129.1,128.5,127.9,127.6,126.4,126.4,125.3,125.2$, 92.6, 56.2, 50.5, 47.9, 44.8, 44.3, 44.3, 37.9, 36.5, 35.8, 31.5, 29.3, 29.0, 29.0, 26.4, 25.5, 25.4, 21.5, 15.4, 13.8; HRMS: (ESI) calcd for $\mathrm{C}_{33} \mathrm{H}_{36} \mathrm{NaO}_{3}{ }^{+}[\mathrm{M}+\mathrm{Na}]^{+}$503.2556; found 
503.2565 .

3a-hydroxy-6a-methyl-5-phenyl-4-(trimethylsilyl)-3,3a,6,6a-tetrahydropentalen $-1(2 H)$-one (3ra)<smiles>CC1CC(=O)C(O)C2CCC(=O)C(O)C12c1ccccc1</smiles>

Chemical Formula: $\mathrm{C}_{18} \mathrm{H}_{24} \mathrm{O}_{2} \mathrm{Si}$

Exact Mass: 300.1546

3ra was prepared according to general procedure 2.1 using $\mathbf{1 r}$ and $\mathbf{2 a}$ and was purified by silica gel column chromatography $(P E / E A=5 / 1)$ to obtain 3ra as yellow solid (9 mg, 15\% yield). $\left.{ }^{1} \mathrm{H} \mathrm{NMR} \mathrm{(400} \mathrm{MHz,} \mathrm{CDCl}_{3}\right) \delta$ 7.33-7.26 (m, 3H), 7.12-7.05 (m, 2H), $2.84(\mathrm{~d}, J=17.2 \mathrm{~Hz}, 1 \mathrm{H}), 2.73(\mathrm{~d}, J=17.3 \mathrm{~Hz}, 1 \mathrm{H})$, 2.57-2.44 (m, 2H), 2.26-2.10 (m, 2H), $1.62(\mathrm{~s}, 1 \mathrm{H}), 1.14(\mathrm{~s}, 3 \mathrm{H}), 0.00(\mathrm{~s}, 9 \mathrm{H})$; ${ }^{13} \mathrm{C}$ NMR $\left(101 \mathrm{MHz}, \mathrm{CDCl}_{3}\right) \delta 221.7,158.9,142.0,139.5,128.0,127.6,127.2$, 95.7, 58.0, 49.8, 37.0, 31.9, 15.6, 0.8; HRMS: (ESI) calcd for $\mathrm{C}_{18} \mathrm{H}_{24} \mathrm{NaO}_{2} \mathrm{Si}^{+}$ $[\mathrm{M}+\mathrm{Na}]^{+}$323.1438; found 323.1430.

8a-benzyl-3a-hydroxy-2,3-diphenyl-3a,8a-dihydrocyclopenta[a]inden-8(1H)-on e (3ua)

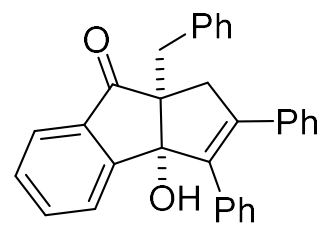

Chemical Formula: $\mathrm{C}_{31} \mathrm{H}_{24} \mathrm{O}_{2}$

Exact Mass: 428.1776

3ua was prepared according to general procedure 2.1 using $\mathbf{1 u}$ and $\mathbf{2 a}$ and was purified by silica gel column chromatography $(P E / E A=10 / 1 \sim 5 / 1)$ to obtain 3ua as white solid $\left(80.5 \mathrm{mg}, 94 \%\right.$ yield). ${ }^{1} \mathrm{H}$ NMR $\left(400 \mathrm{MHz}, \mathrm{CDCl}_{3}\right) \delta$ 7.71-7.67 (m, 1H), 7.35-7.29 (m, 2H), 7.25-7.22 (m, 4H), 7.20-7.10 (m, 4H), 
7.10-7.04 (m, 4H), 7.03-6.96 (m, 4H), 3.42-3.34 (m, 2H), 3.12 (d, $J=13.7 \mathrm{~Hz}$, $1 \mathrm{H}), 3.06(\mathrm{~d}, J=17.3 \mathrm{~Hz}, 1 \mathrm{H}), 2.81(\mathrm{~s}, 1 \mathrm{H}) ;{ }^{13} \mathrm{C} \mathrm{NMR}\left(101 \mathrm{MHz}, \mathrm{CDCl}_{3}\right) \delta$ 206.9, 154.4, 141.1, 139.9, 137.1, 135.6, 135.5, 134.2, 130.3, 129.9, 129.0, 128.3, 128.1, 127.9, 127.8, 127.6, 127.3, 126.4, 124.7, 123.3, 93.1, 63.7, 41.4, 39.1; HRMS: (ESI) calcd for $\mathrm{C}_{31} \mathrm{H}_{24} \mathrm{NaO}_{2}{ }^{+}[\mathrm{M}+\mathrm{Na}]^{+} 451.1669$; found 451.1648 .

7a-hydroxy-3a,6,6-trimethyl-1,2-diphenyl-3,3a,5,6,7,7a-hexahydro-4H-inden-4 -one (3va)<smiles>CC1(C)CC(=O)C2(C)CC(c3ccccc3)=C(c3ccccc3)C(O)(C1)C2</smiles>

Chemical Formula: $\mathrm{C}_{24} \mathrm{H}_{26} \mathrm{O}_{2}$

Exact Mass: 346.1933

3va was prepared according to general procedure 2.1 using $1 \mathbf{v}$ and $2 \mathrm{a}$ and was purified by silica gel column chromatography $(P E / E A=5 / 1)$ to obtain 3va as yellow oil (27.7 mg, $40 \%$ yield). ${ }^{1} \mathrm{H}$ NMR $\left(400 \mathrm{MHz}, \mathrm{CDCl}_{3}\right) \delta 7.29-7.26(\mathrm{~m}$, $2 \mathrm{H}), 7.26-7.24(\mathrm{~m}, 1 \mathrm{H}), 7.24-7.20(\mathrm{~m}, 2 \mathrm{H}), 7.18-7.11(\mathrm{~m}, 5 \mathrm{H}), 3.67(\mathrm{~d}, J=16.6$ $\mathrm{Hz}, 1 \mathrm{H}), 2.58(\mathrm{~d}, J=13.2 \mathrm{~Hz}, 1 \mathrm{H}), 2.47(\mathrm{~d}, J=16.6 \mathrm{~Hz}, 1 \mathrm{H}), 2.22(\mathrm{dd}, J=13.2$, $1.4 \mathrm{~Hz}, 1 \mathrm{H}$ ), 2.07 (dd, $J=14.4,1.5 \mathrm{~Hz}, 1 \mathrm{H}$ ), 1.87 (d, $J=14.4 \mathrm{~Hz}, 1 \mathrm{H}$ ), 1.79 (s, $1 \mathrm{H}), 1.40(\mathrm{~s}, 3 \mathrm{H}), 0.99(\mathrm{~s}, 3 \mathrm{H}), 0.69(\mathrm{~s}, 3 \mathrm{H}) ;{ }^{13} \mathrm{C} \mathrm{NMR}\left(101 \mathrm{MHz}, \mathrm{CDCl}_{3}\right) \delta$ $213.8,142.1,139.5,136.3,135.7,129.5,128.4,128.2,128.0,127.4,127.1$, 89.7, 57.5, 50.6, 44.4, 43.1, 32.7, 30.9, 28.3, 19.1; HRMS: (ESI) calcd for $\mathrm{C}_{24} \mathrm{H}_{26} \mathrm{NaO}_{2}{ }^{+}[\mathrm{M}+\mathrm{Na}]^{+} 369.1825$; found 369.1826 .

1-methyl-2,3-diphenylcyclopent-2-en-1-ol (3wa) 


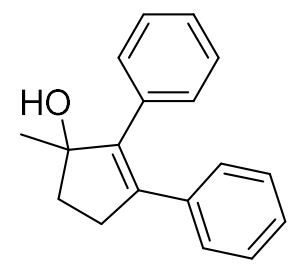

Chemical Formula: $\mathrm{C}_{18} \mathrm{H}_{18} \mathrm{O}$

Exact Mass: 250.1358

3wa was prepared according to general procedure 2.1 using $1 \mathbf{w}$ and $2 \mathbf{a}$ and was purified by silica gel column chromatography $(P E / E A=10 / 1 \sim 5 / 1)$ to obtain 3wa as yellow oil $\left(35.0 \mathrm{mg}, 70 \%\right.$ yield). ${ }^{1} \mathrm{H}$ NMR $\left(400 \mathrm{MHz},\left(\mathrm{CD}_{3}\right)_{2} \mathrm{CO}\right) \delta$ 7.30-7.21 (m, 5H), $7.13(\mathrm{~s}, 5 \mathrm{H}), 3.94(\mathrm{~s}, 1 \mathrm{H}), 2.87-2.74(\mathrm{~m}, 2 \mathrm{H}), 2.26-2.11(\mathrm{~m}$, $2 \mathrm{H}), 1.25$ (s, 3H); ${ }^{13} \mathrm{C} \mathrm{NMR}\left(101 \mathrm{MHz},\left(\mathrm{CD}_{3}\right)_{2} \mathrm{CO}\right) \delta$ 145.2, 138.7, 138.4, 138.3, 130.6, 129.0, 128.7, 128.6, 127.6, 127.5, 85.5, 40.9, 33.4, 26.7; HRMS: (ESI) calcd for $\mathrm{C}_{18} \mathrm{H}_{18} \mathrm{ONa}{ }^{+}[\mathrm{M}+\mathrm{Na}]^{+} 273.1257$; found 273.1250 .

4-methoxy-3'-methyl-3',4',5',6'-tetrahydro-[1,1':2', 1'-terphenyl]-3'-ol (3xb)

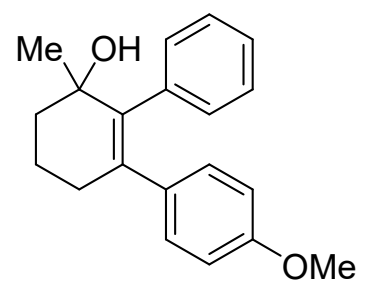

Chemical Formula: $\mathrm{C}_{20} \mathrm{H}_{22} \mathrm{O}_{2}$

Exact Mass: 294.1620

$\mathbf{3} \mathbf{x b}$ was prepared according to general procedure 2.1 using $\mathbf{1 x}$ and $\mathbf{2 b}$ and was purified by silica gel column chromatography $(P E / E A=30 / 1 \sim 10 / 1)$ to obtain $3 \times$ b as yellow oil $\left(23.5 \mathrm{mg}, 80 \%\right.$ yield). ${ }^{1} \mathrm{H}$ NMR $\left(600 \mathrm{MHz},\left(\mathrm{CD}_{3}\right)_{2} \mathrm{SO}\right) \delta$ 7.09-7.00 (m, 5H), 6.85-6.82 (m, 2H), 6.60-6.57 (m, 2H), $4.55(\mathrm{~s}, 1 \mathrm{H}), 3.61(\mathrm{~s}$, $3 \mathrm{H}), 2.49-2.43(\mathrm{~m}, 1 \mathrm{H}), 2.20-2.14(\mathrm{~m}, 1 \mathrm{H}), 2.00-1.94(\mathrm{~m}, 1 \mathrm{H}), 1.88-1.82(\mathrm{~m}$, 1H), 1.78-1.68 (m, 2H), $0.97(\mathrm{~s}, 3 \mathrm{H}) ;{ }^{13} \mathrm{C}$ NMR (151 MHz, $\left.\left(\mathrm{CD}_{3}\right)_{2} \mathrm{SO}\right) \delta$ 156.9, $140.4,140.2$, 135.7, 135.5, 130.9, 129.4, 126.7, 125.4, 112.8, 68.6, 54.7, 32.5, 28.8, 19.0; HRMS: (ESI) calcd for $\mathrm{C}_{20} \mathrm{H}_{22} \mathrm{O}_{2} \mathrm{Na}^{+}[\mathrm{M}+\mathrm{Na}]^{+} 317.1512$; found 317.1505 . 
1,2-diphenyl-5H-pyrrolo[2,1-a]isoindol-5-one (3ya)<smiles>O=C1c2ccccc2-c2c(-c3ccccc3)c(-c3ccccc3)cn21</smiles>

Chemical Formula: $\mathrm{C}_{23} \mathrm{H}_{15} \mathrm{NO}$

Exact Mass: 321.1154

3ya was prepared according to general procedure 2.1 using $\mathbf{1 y}$ and $\mathbf{2 a}$ and was purified by silica gel column chromatography $(P E / E A=5 / 1)$ to obtain 3ya as yellow solid (23.1 mg, $36 \%$ yield). ${ }^{1} \mathrm{H}$ NMR $\left(400 \mathrm{MHz}, \mathrm{CDCl}_{3}\right) \delta 7.73-7.64$ (m, 1H), 7.41-7.32 (m, 6H), 7.30-7.27 (m, 1H), 7.26-7.22 (m, 3H), 7.22-7.14 (m, $4 \mathrm{H}) ;{ }^{13} \mathrm{C}$ NMR $\left(101 \mathrm{MHz}, \mathrm{CDCl}_{3}\right) \delta 162.9,136.3,134.4,133.4,132.9,132.9$, $132.4,131.8,129.5,128.5,128.3,128.1,127.7,127.4,127.1,125.8,124.3$, 119.5, 114.6; HRMS: (ESI) calcd for $\mathrm{C}_{23} \mathrm{H}_{16} \mathrm{NO}^{+}[\mathrm{M}+\mathrm{H}]^{+} 322.1264$; found 322.1231. 


\section{Copies of the ${ }^{1} \mathrm{H},{ }^{19} \mathrm{~F}$ and ${ }^{13} \mathrm{C}$ NMR spectra}

1g (600 MHz for ${ }^{1} \mathrm{H}$ NMR with $\mathrm{CDCl}_{3}$ as solvent)

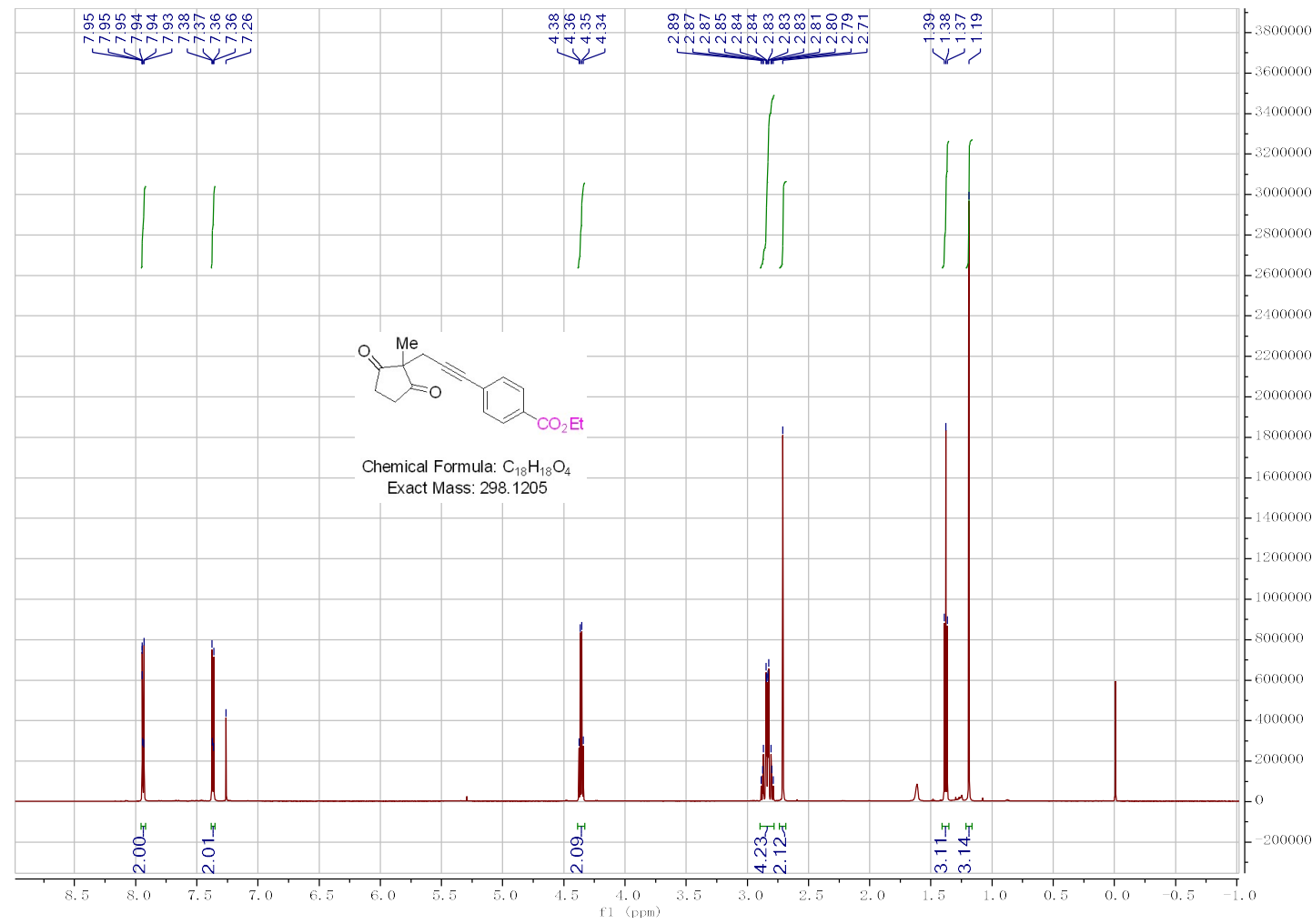

$1 \mathbf{g}\left(151 \mathrm{MHz}\right.$ for ${ }^{13} \mathrm{C}$ NMR with $\mathrm{CDCl}_{3}$ as solvent)

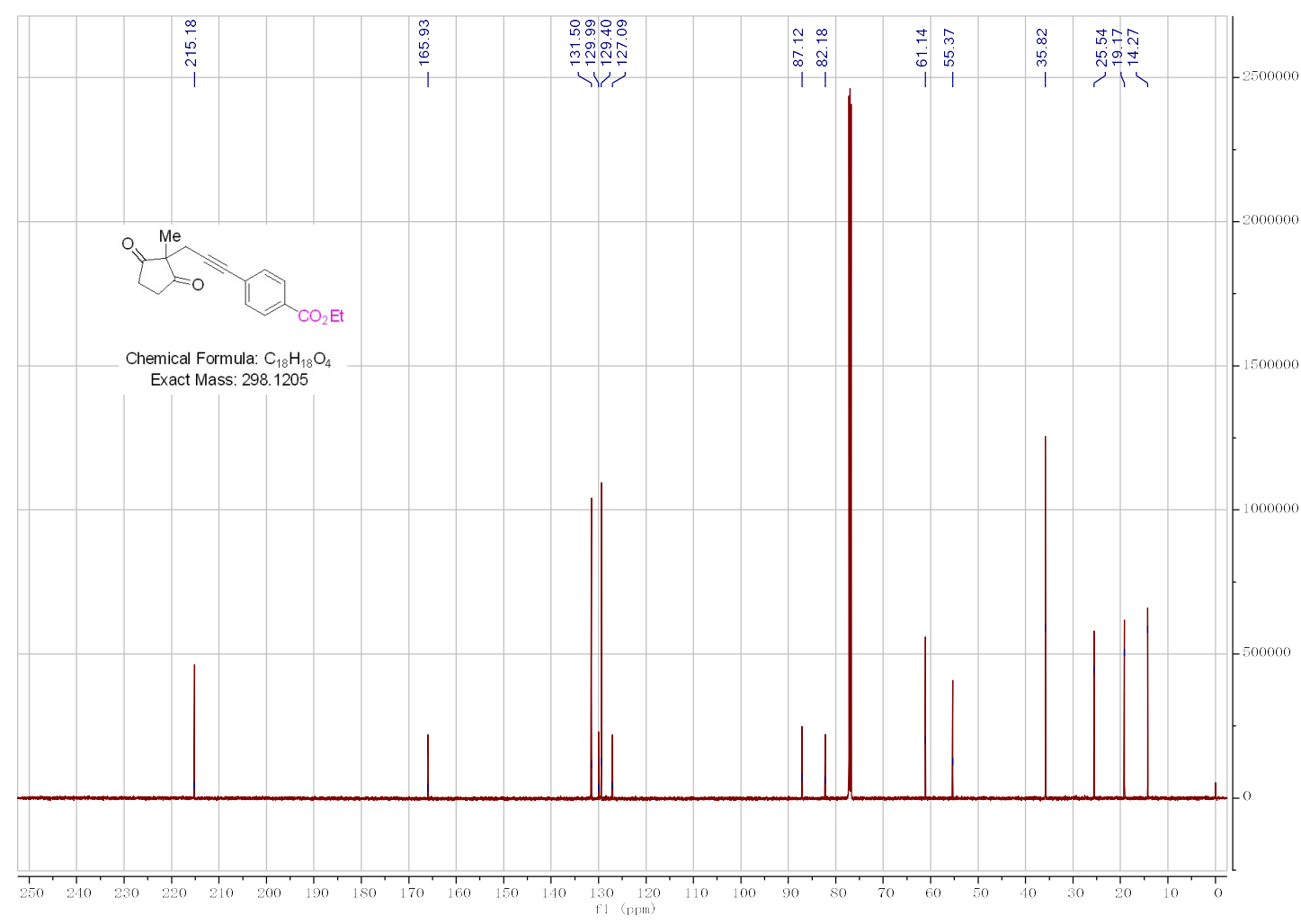


1h (600 MHz for ${ }^{1} \mathrm{H} \mathrm{NMR}$ with $\mathrm{CDCl}_{3}$ as solvent)

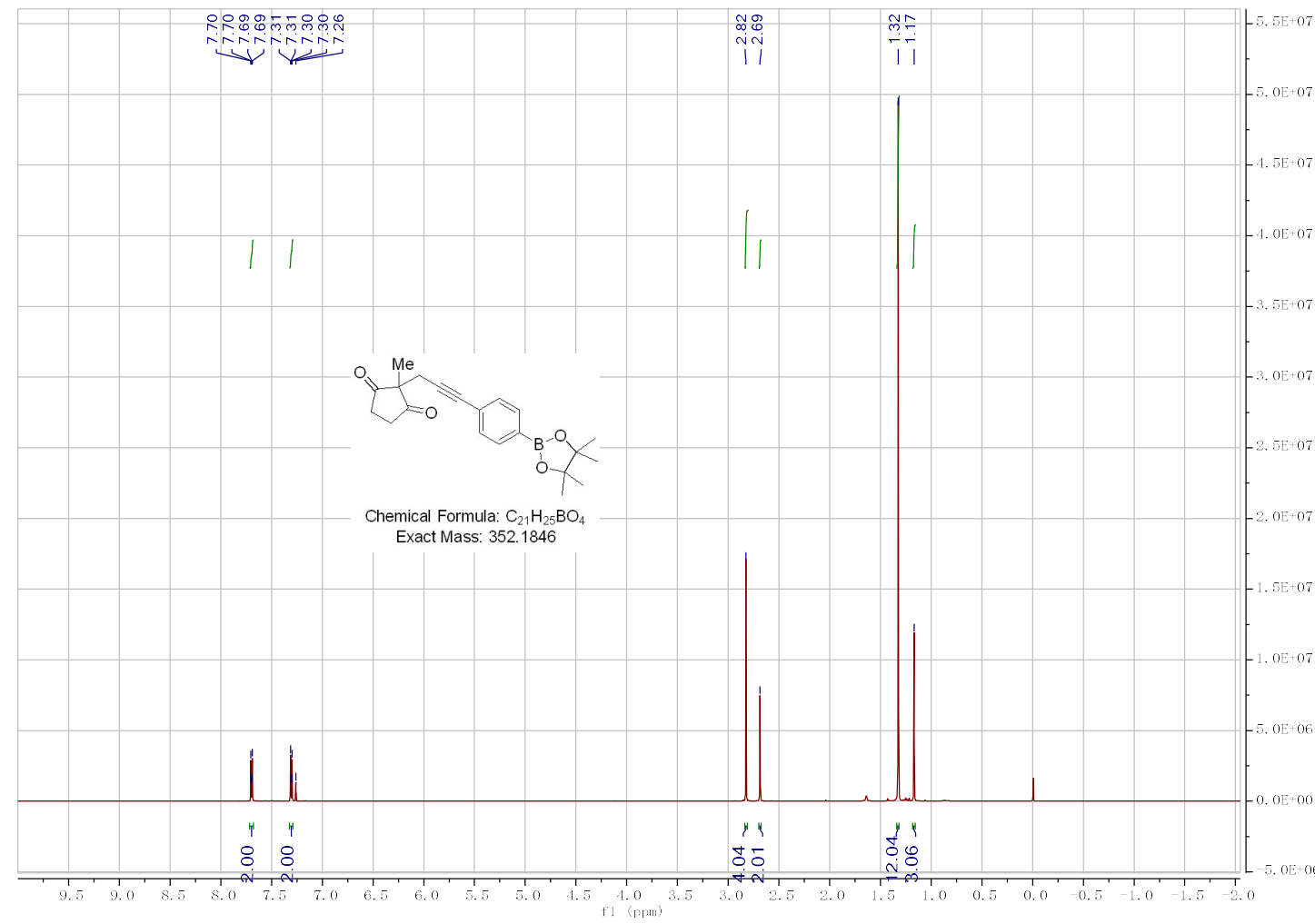

1h (151 MHz for ${ }^{13} \mathrm{C}$ NMR with $\mathrm{CDCl}_{3}$ as solvent)

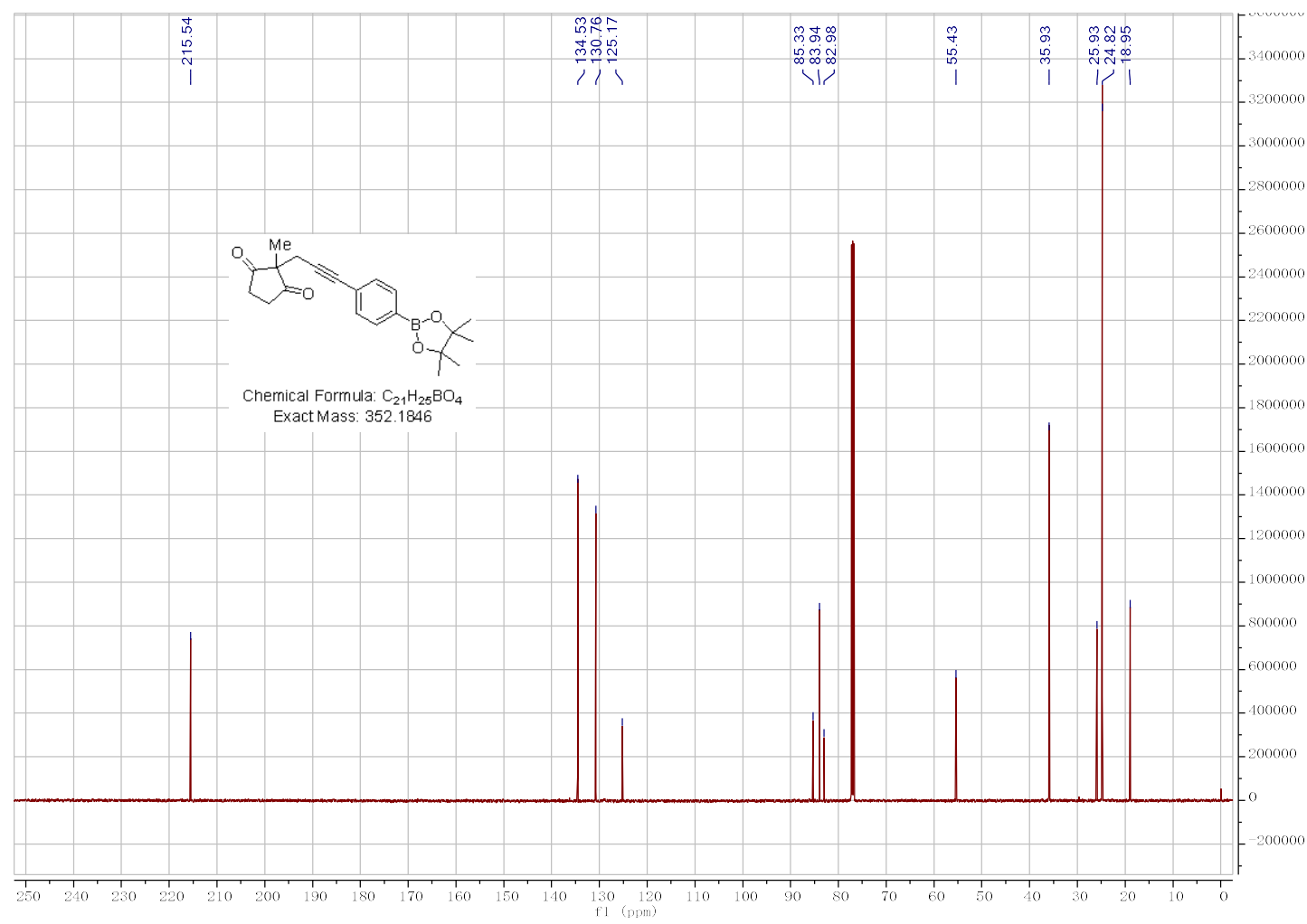


1i (600 MHz for ${ }^{1} \mathrm{H}$ NMR with $\mathrm{CDCl}_{3}$ as solvent)

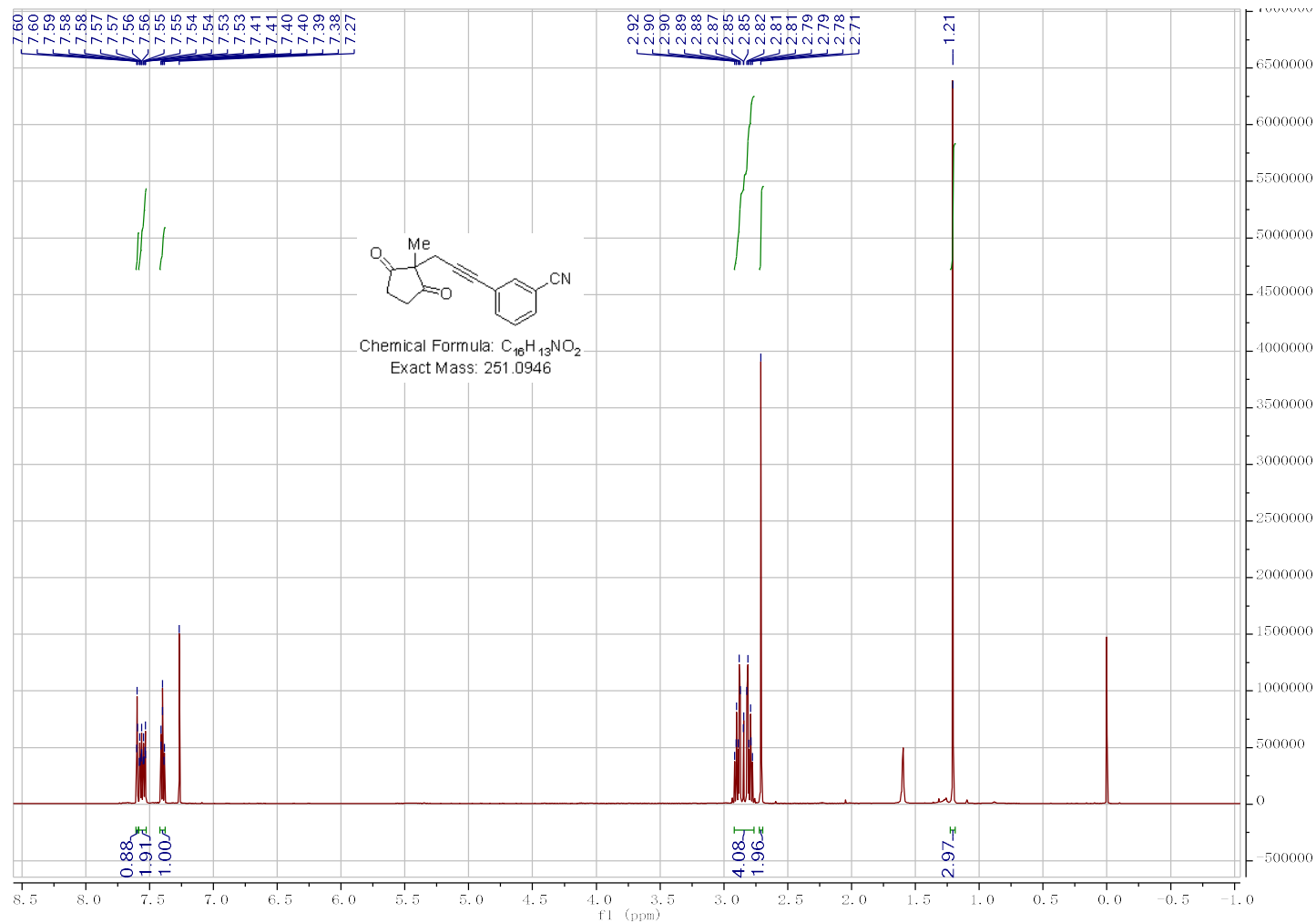

1i (151 MHz for ${ }^{13} \mathrm{C} \mathrm{NMR}$ with $\mathrm{CDCl}_{3}$ as solvent)

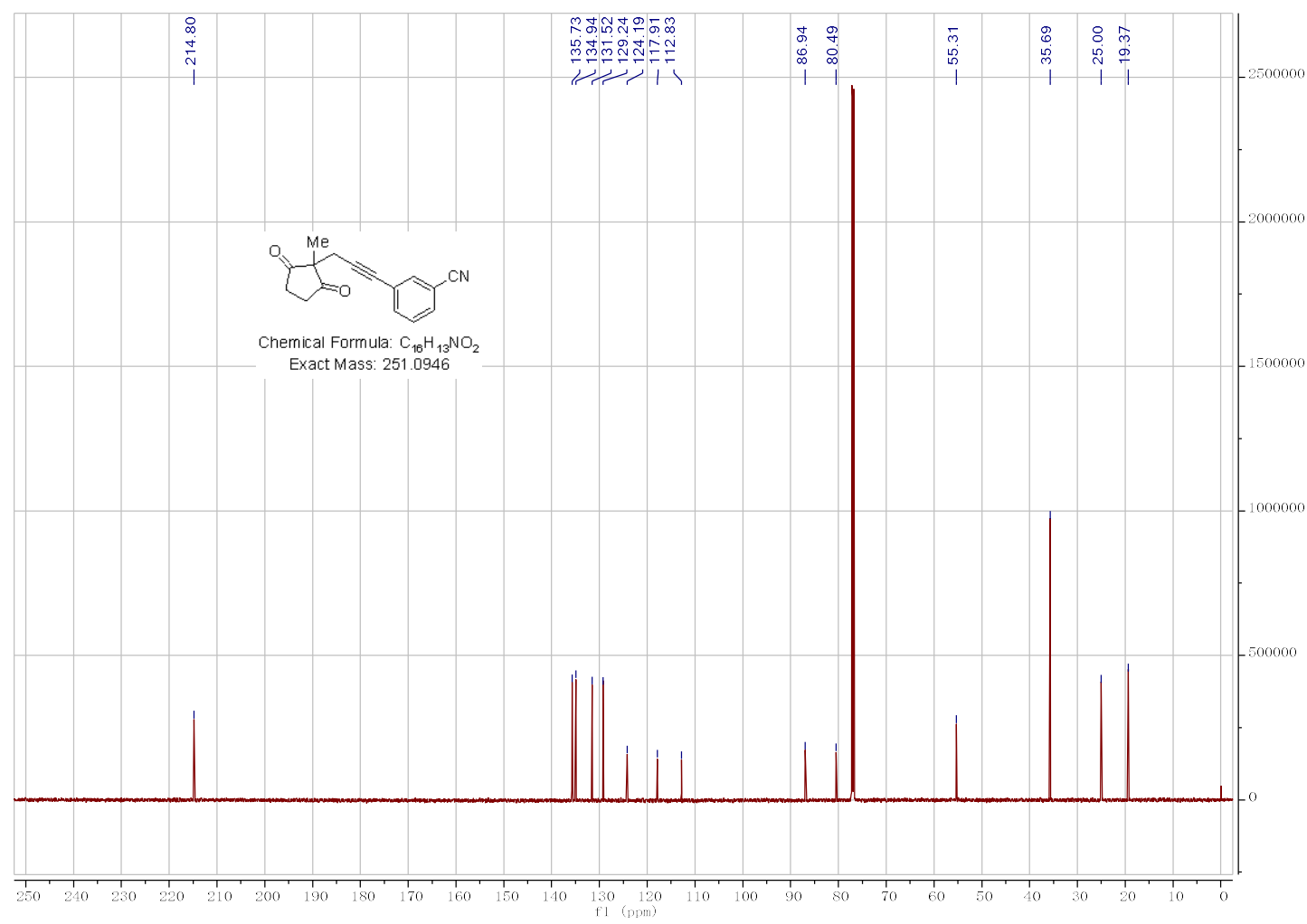


1I (600 MHz for ${ }^{1} \mathrm{H} \mathrm{NMR}$ with $\mathrm{CDCl}_{3}$ as solvent)

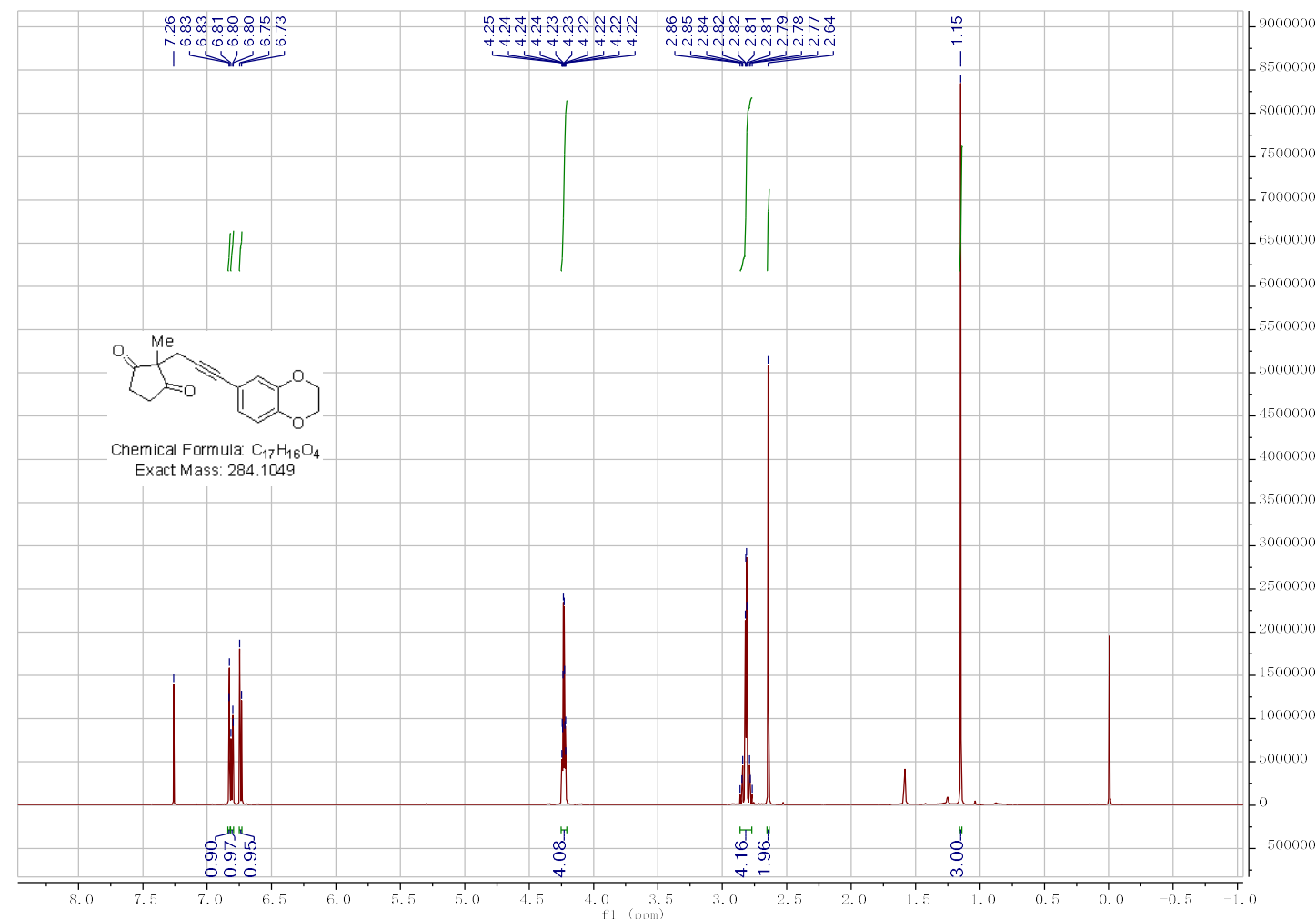

1 ( $151 \mathrm{MHz}$ for ${ }^{13} \mathrm{C} \mathrm{NMR}$ with $\mathrm{CDCl}_{3}$ as solvent)

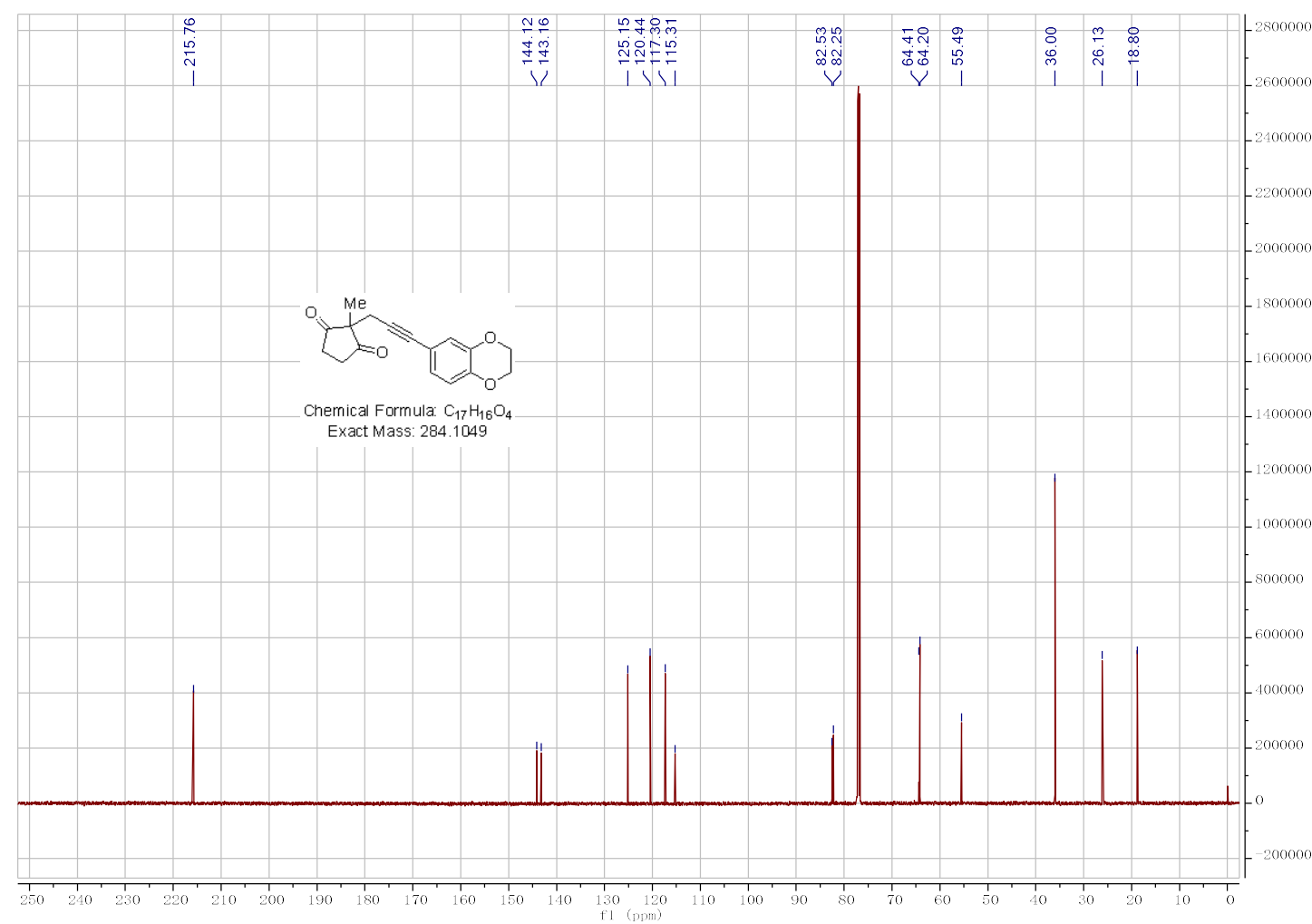


$1 \mathrm{~m}\left(600 \mathrm{MHz}\right.$ for ${ }^{1} \mathrm{H}$ NMR with $\mathrm{CDCl}_{3}$ as solvent)

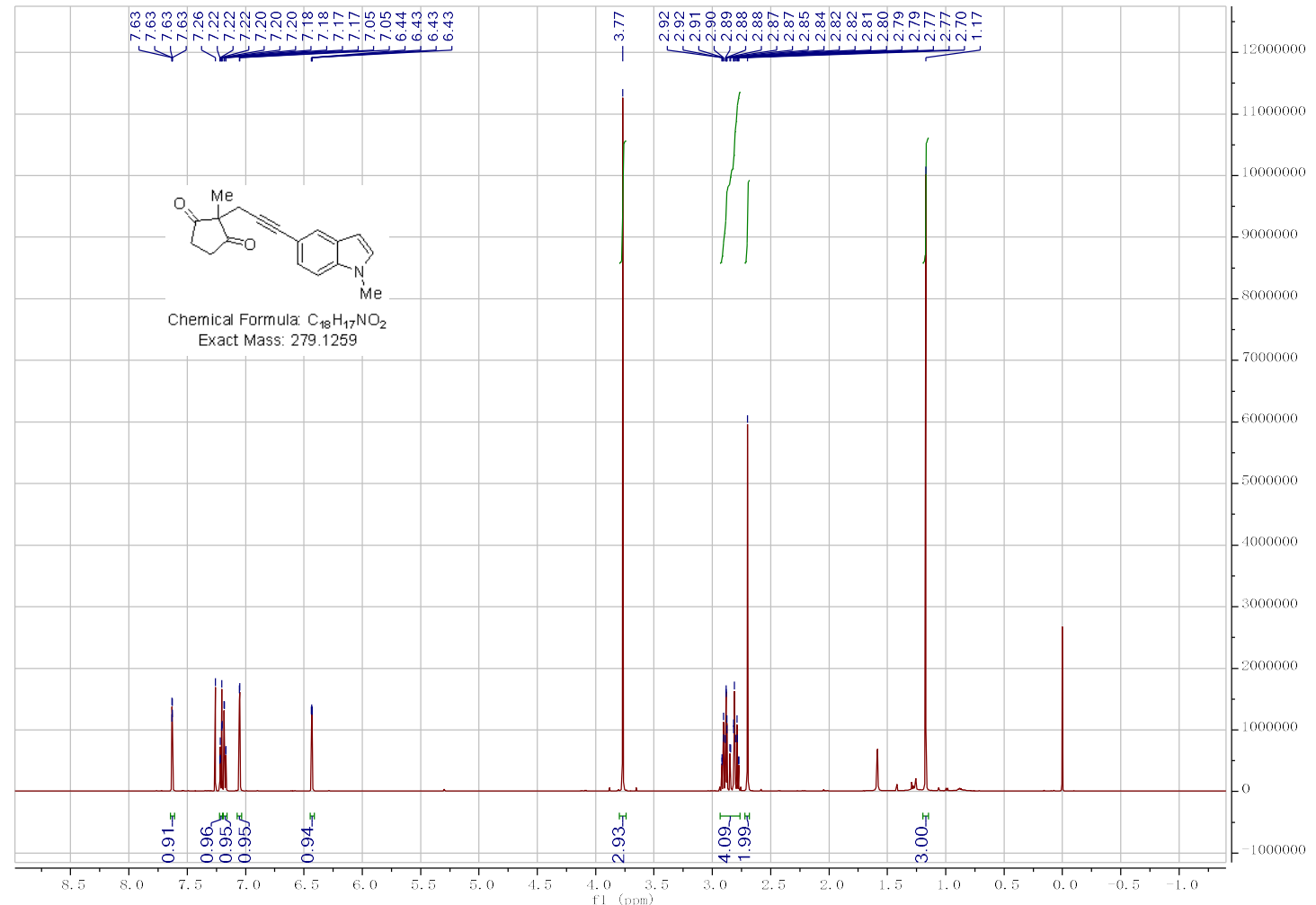

$1 \mathrm{~m}\left(151 \mathrm{MHz}\right.$ for ${ }^{13} \mathrm{C} \mathrm{NMR}$ with $\mathrm{CDCl}_{3}$ as solvent)

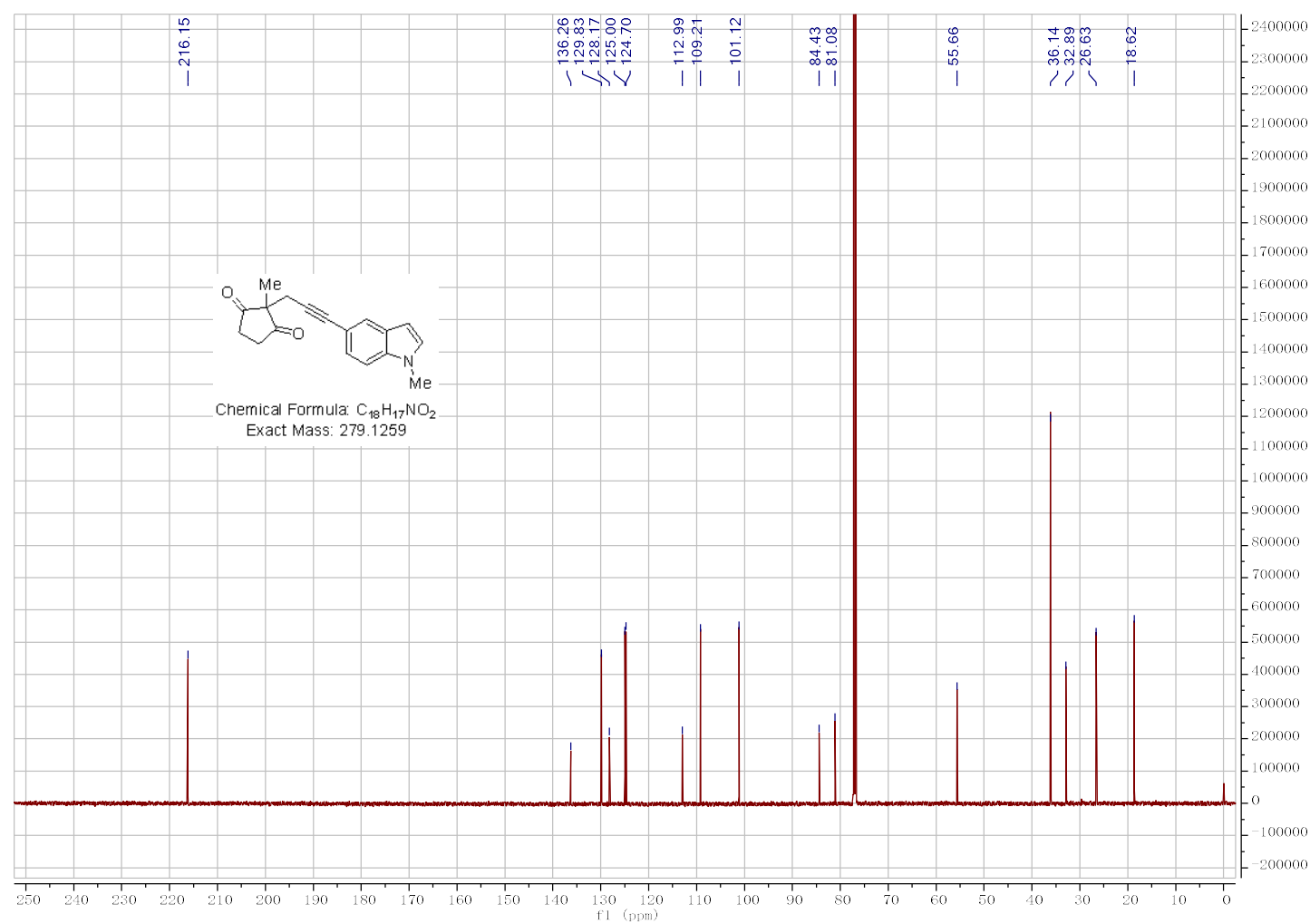


1n (600 MHz for ${ }^{1} \mathrm{H} \mathrm{NMR}$ with $\mathrm{CDCl}_{3}$ as solvent)

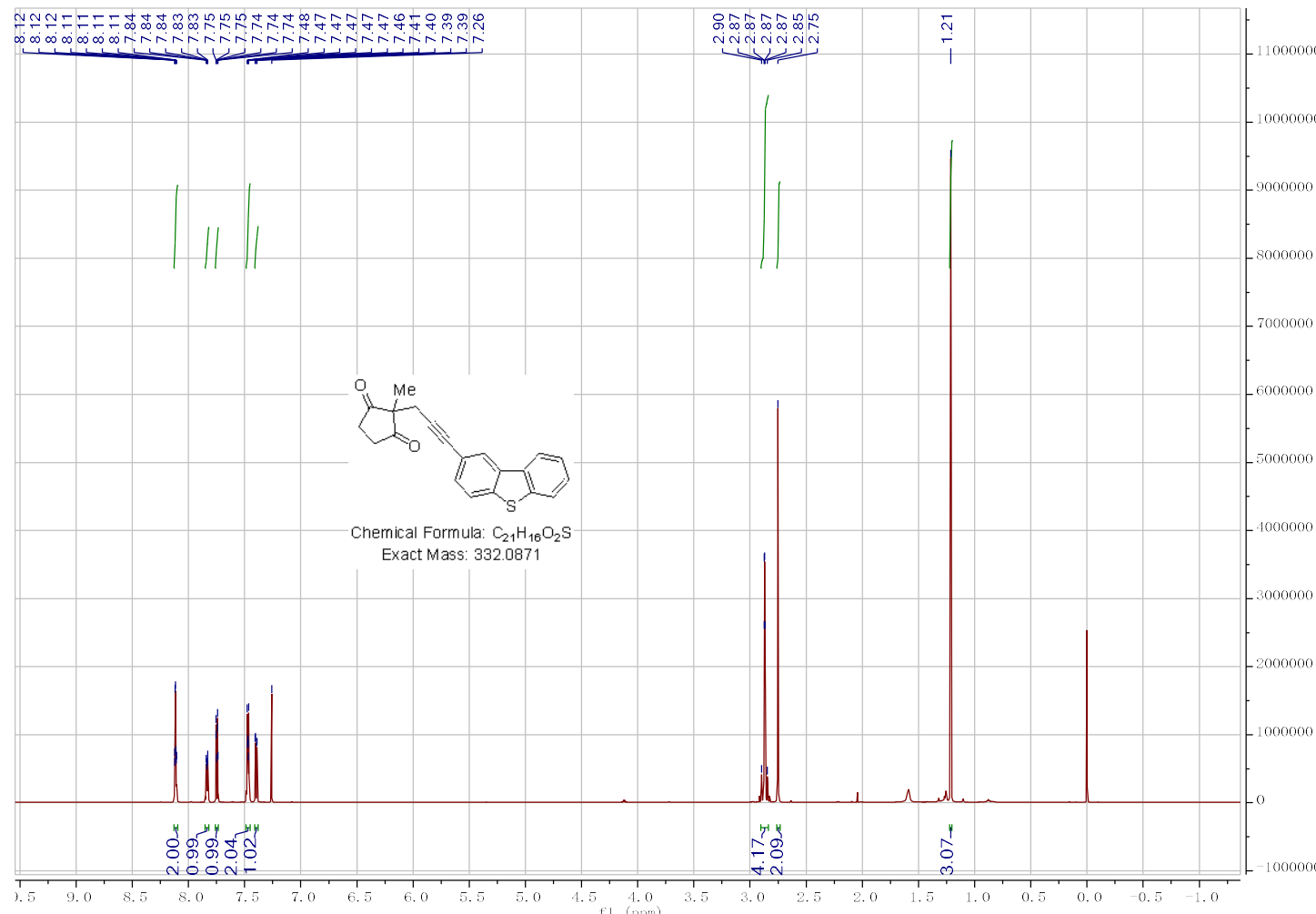

1n (151 MHz for ${ }^{13} \mathrm{C}$ NMR with $\mathrm{CDCl}_{3}$ as solvent)

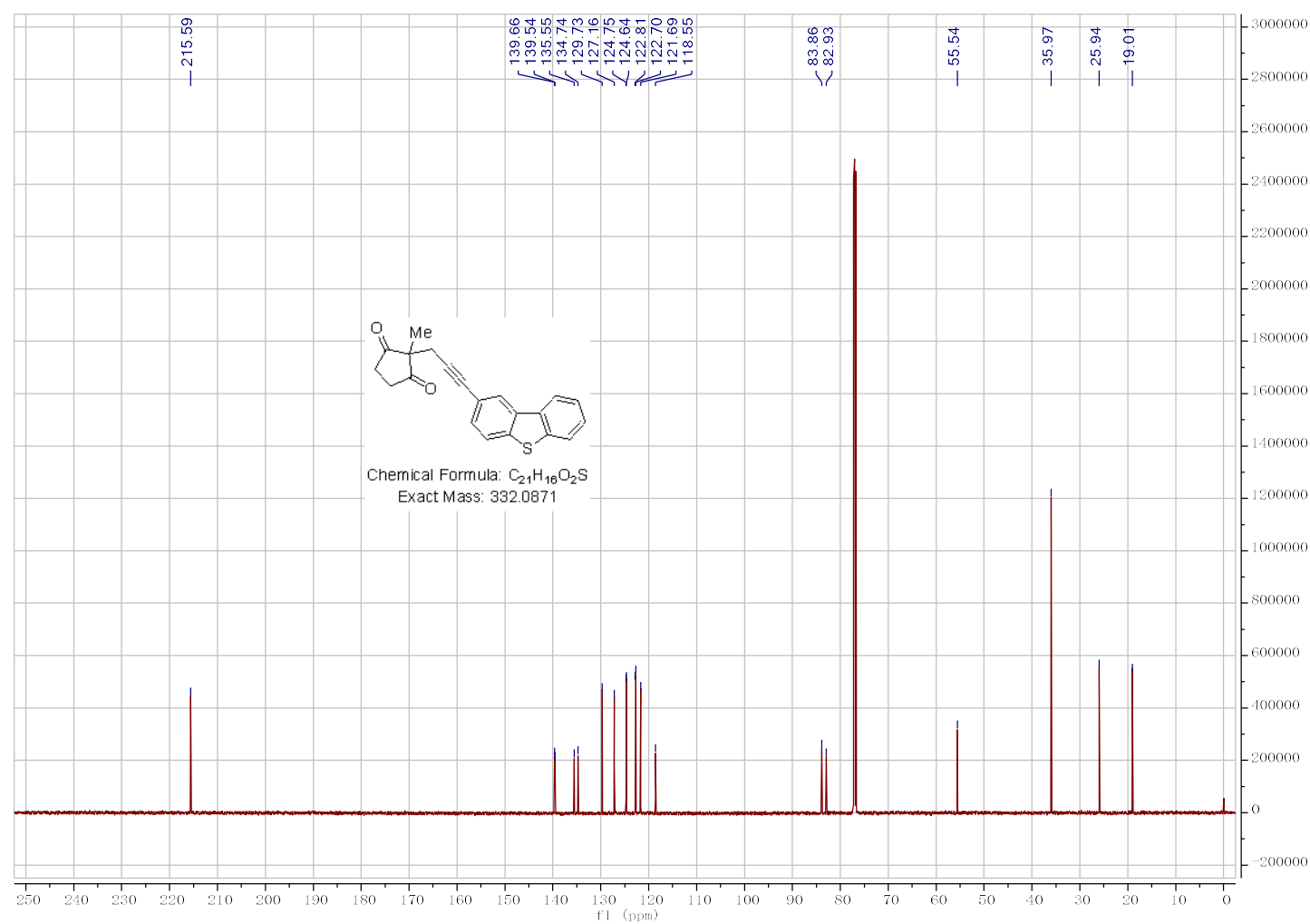


$10\left(600 \mathrm{MHz}\right.$ for ${ }^{1} \mathrm{H} \mathrm{NMR}$ with $\mathrm{CDCl}_{3}$ as solvent)

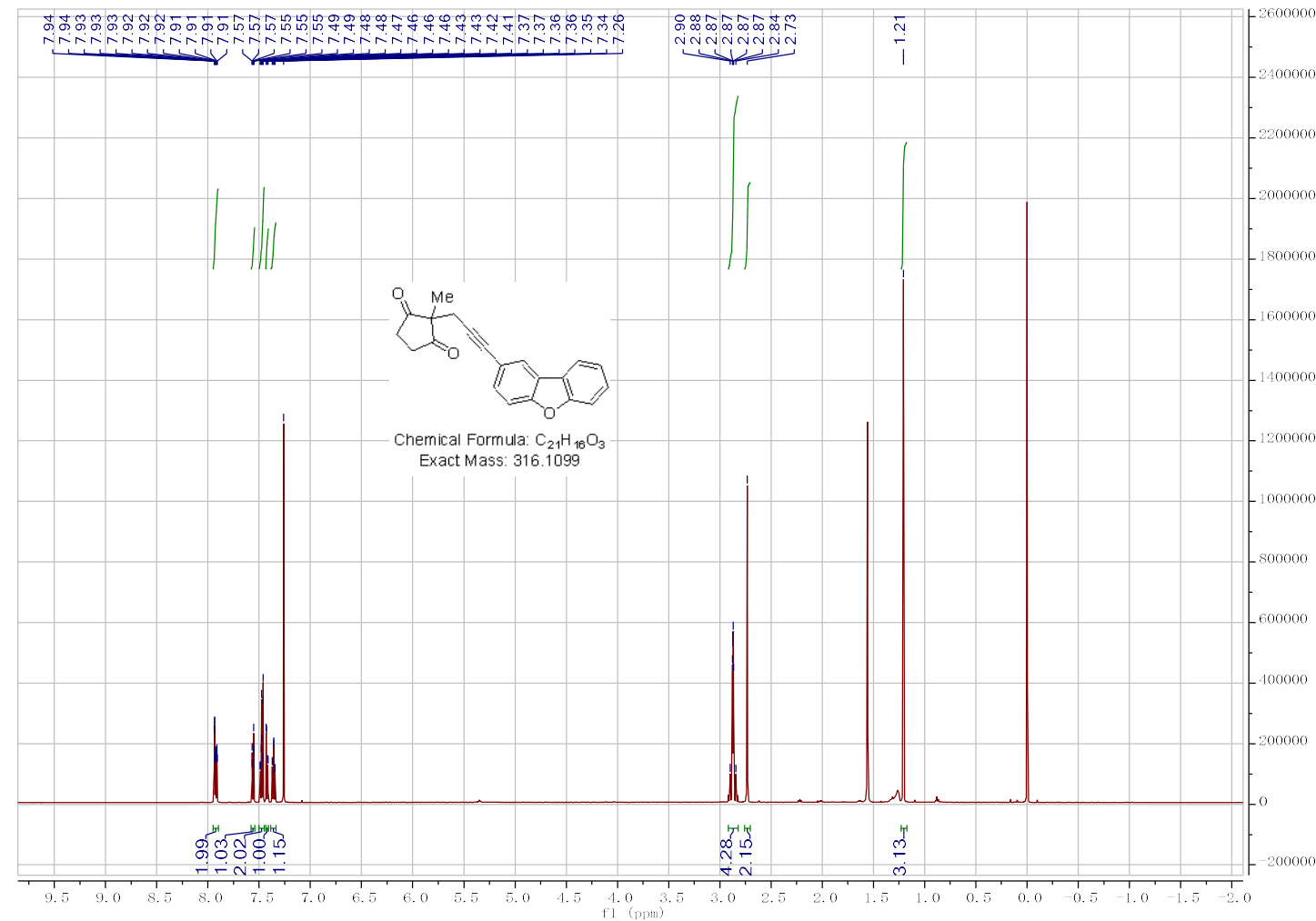

10 (151 MHz for ${ }^{13} \mathrm{C}$ NMR with $\mathrm{CDCl}_{3}$ as solvent)

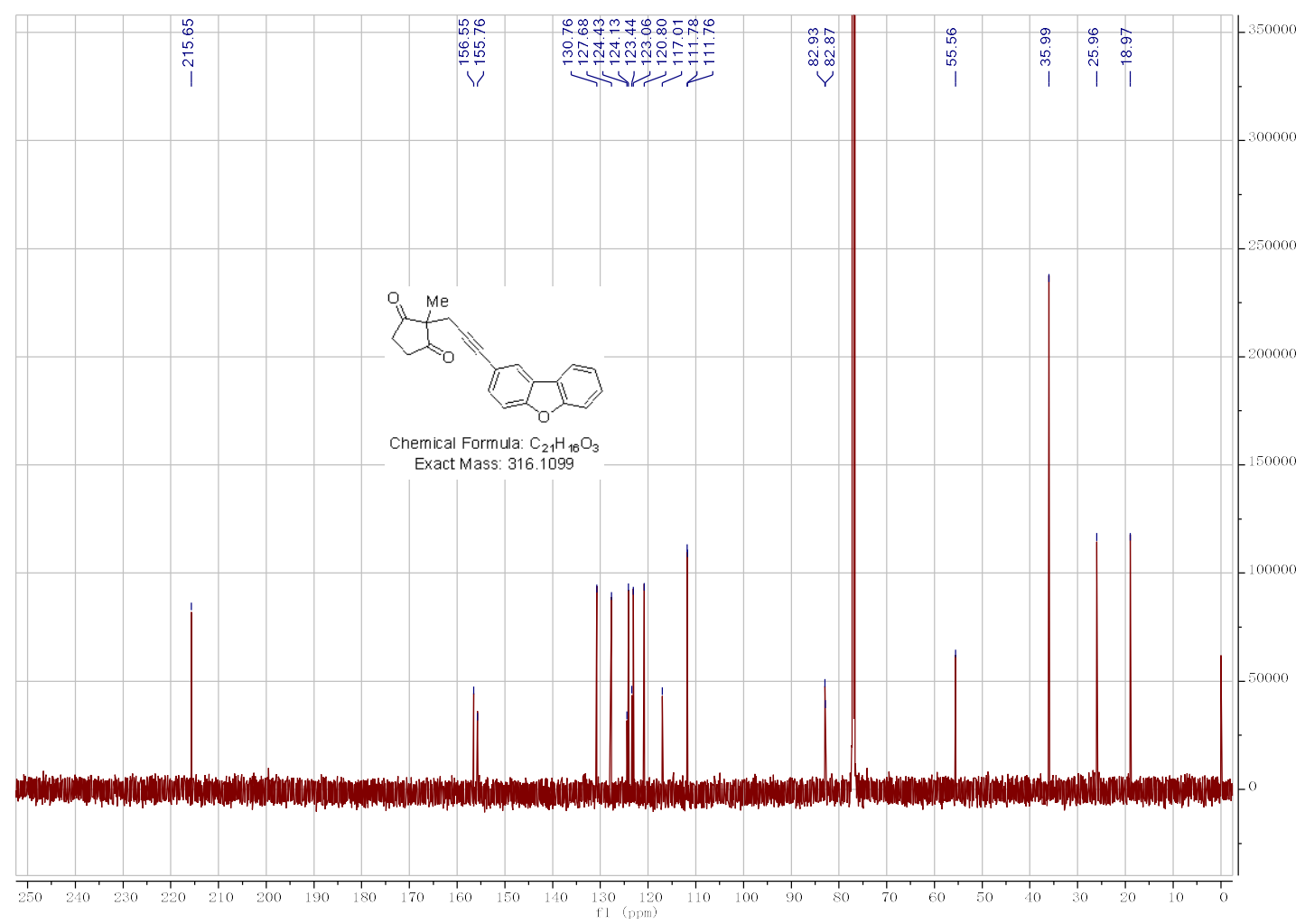


1p (600 MHz for ${ }^{1} \mathrm{H} \mathrm{NMR}$ with $\mathrm{CDCl}_{3}$ as solvent)

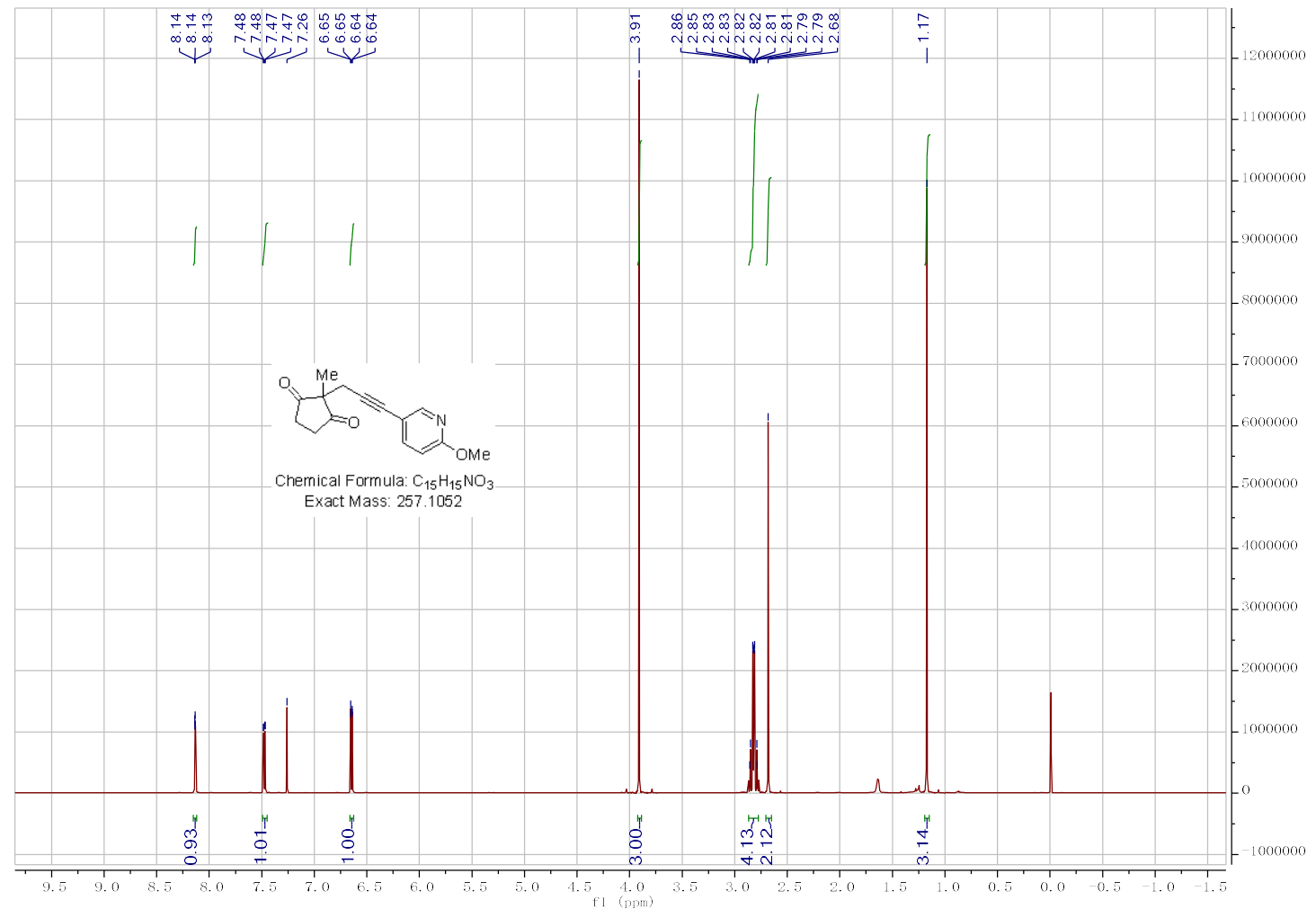

$1 \mathrm{p}\left(151 \mathrm{MHz}\right.$ for ${ }^{13} \mathrm{C} \mathrm{NMR}$ with $\mathrm{CDCl}_{3}$ as solvent)

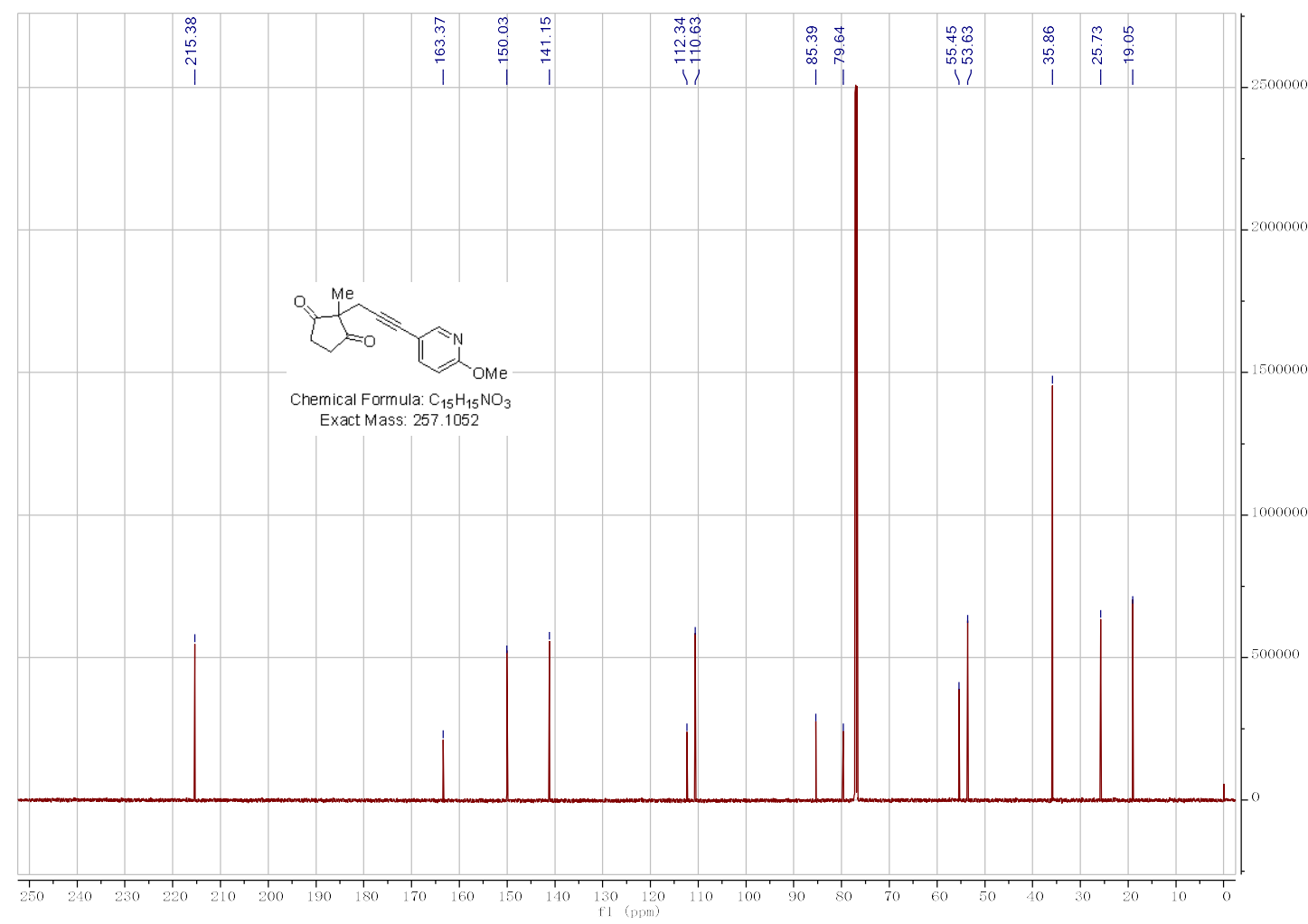


1q $\left(600 \mathrm{MHz}\right.$ for ${ }^{1} \mathrm{H} \mathrm{NMR}$ with $\mathrm{CDCl}_{3}$ as solvent)

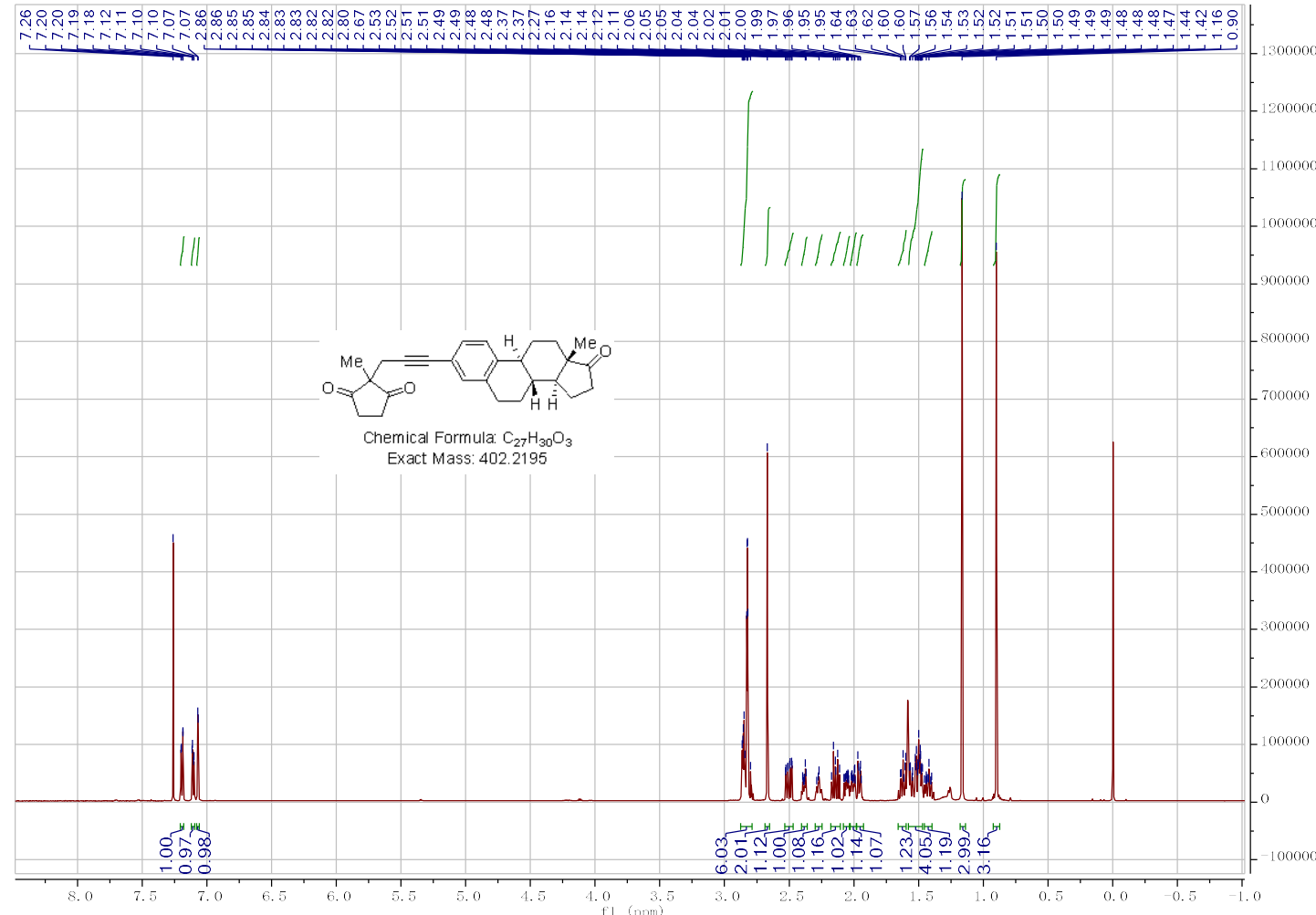

1q (151 MHz for ${ }^{13} \mathrm{C}$ NMR with $\mathrm{CDCl}_{3}$ as solvent)

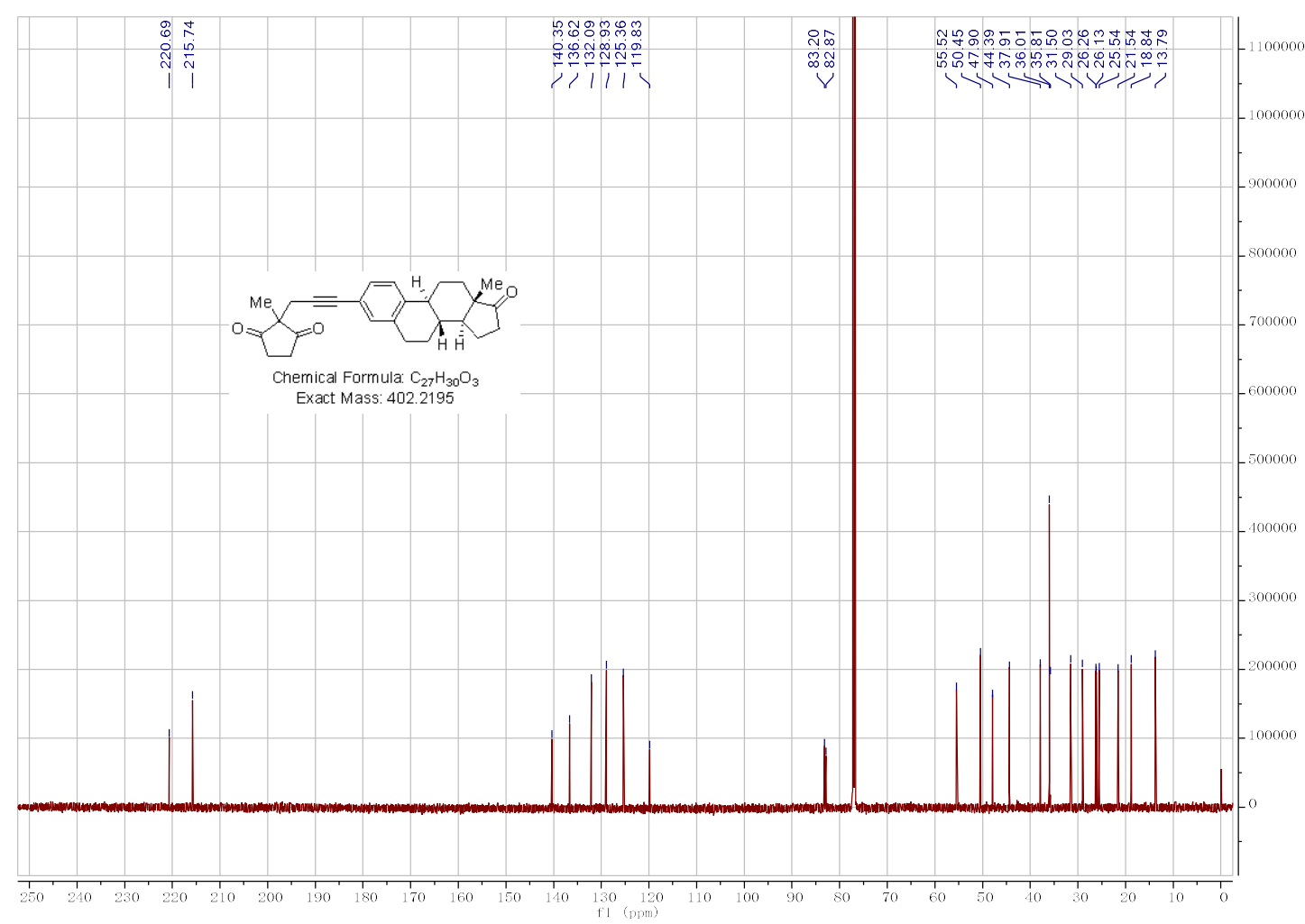


1v (600 MHz for ${ }^{1} \mathrm{H} \mathrm{NMR}$ with $\mathrm{CDCl}_{3}$ as solvent)

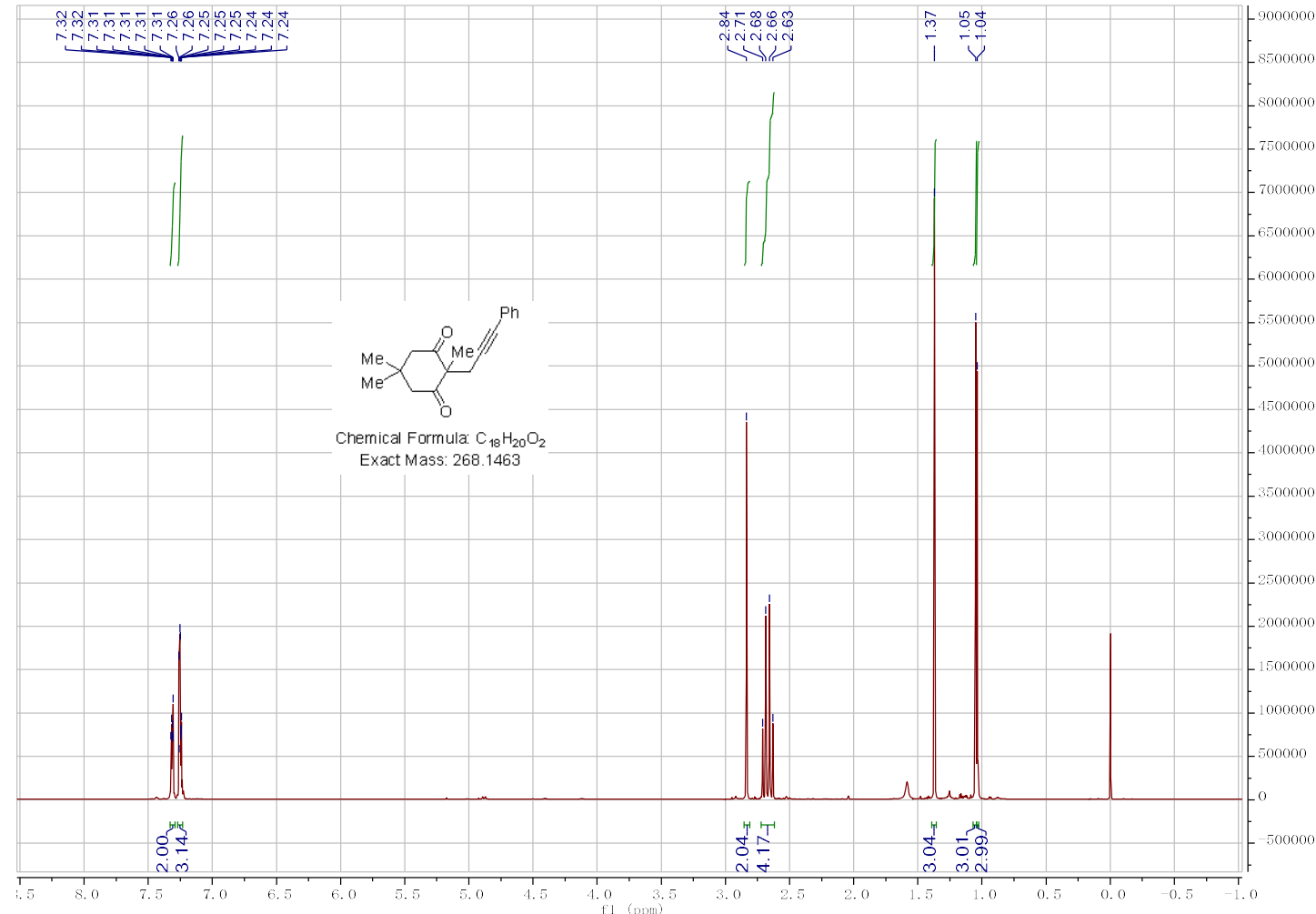

1v (151 MHz for ${ }^{13} \mathrm{C}$ NMR with $\mathrm{CDCl}_{3}$ as solvent)

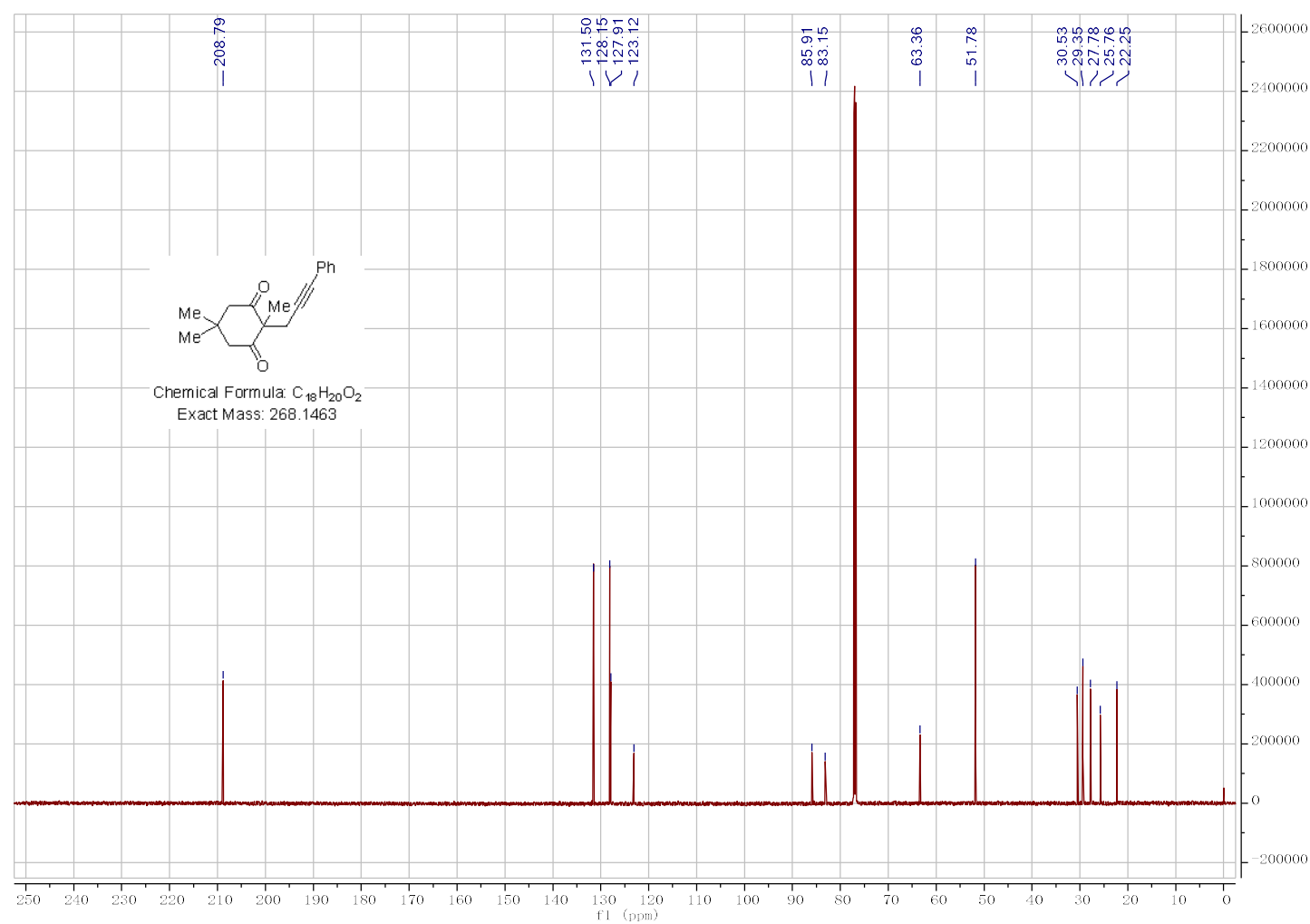


3aa (400 MHz for ${ }^{1} \mathrm{H}$ NMR with $\mathrm{CDCl}_{3}$ as solvent)

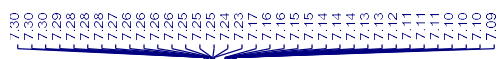

管

H|

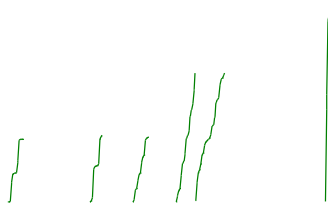

$\sum_{\overline{\mathrm{oH}}}^{\mathrm{Me}}$

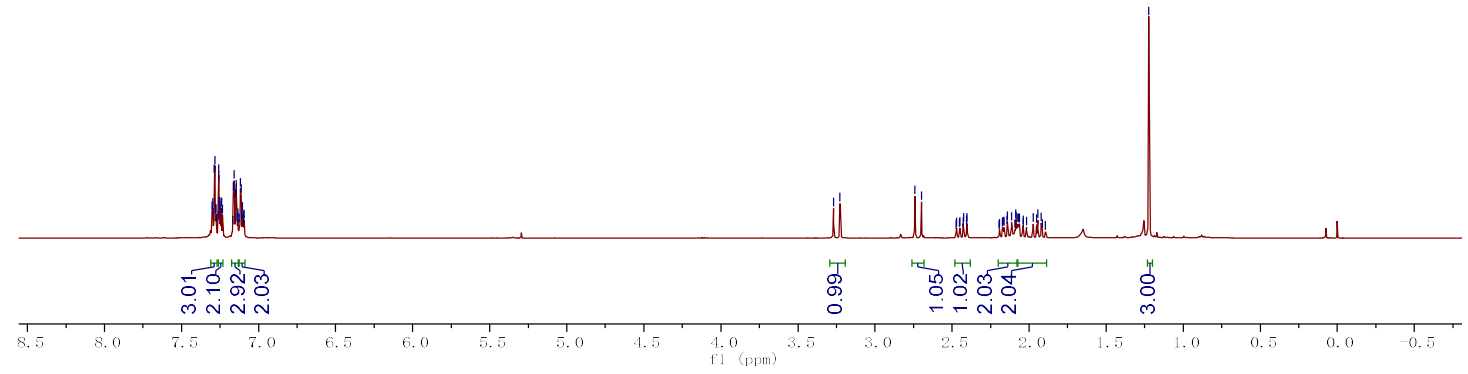

3aa (101 MHz for ${ }^{13} \mathrm{C} \mathrm{NMR}$ with $\mathrm{CDCl}_{3}$ as solvent)

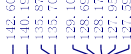

i v
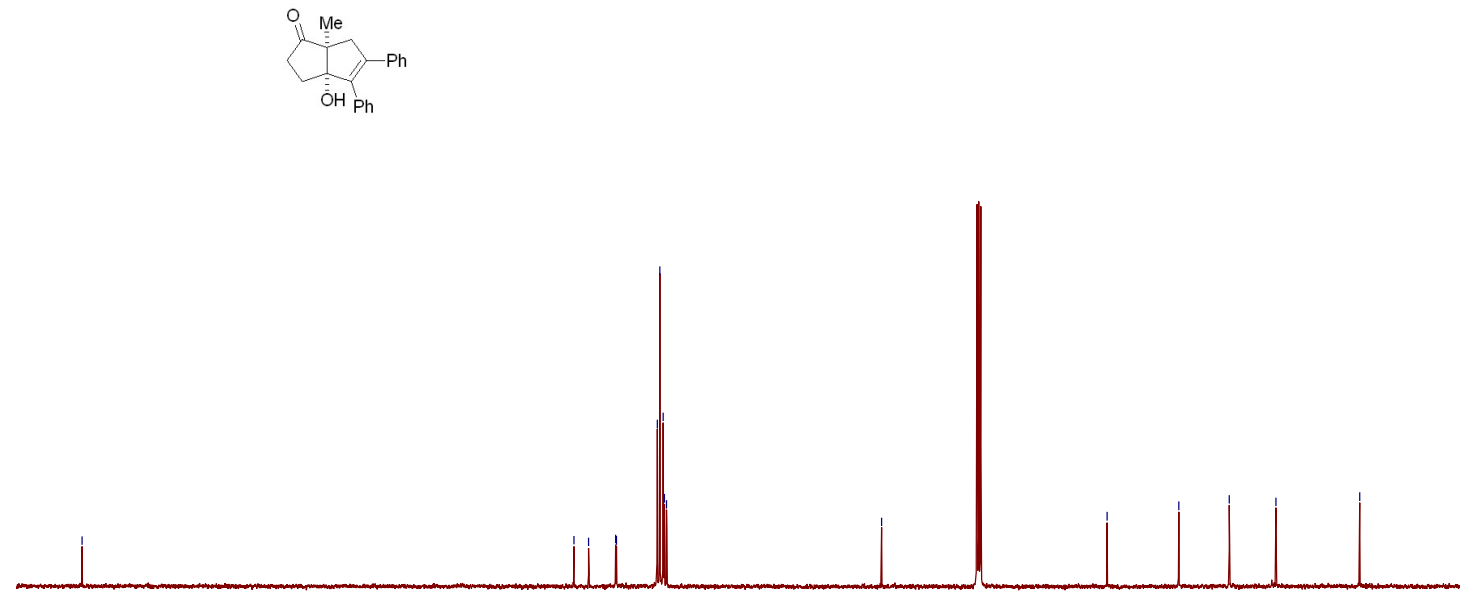

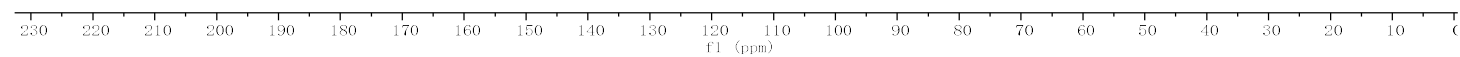


3ab (400 MHz for ${ }^{1} \mathrm{H} \mathrm{NMR}$ with $\mathrm{CDCl}_{3}$ as solvent)

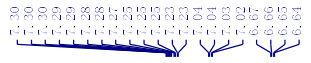

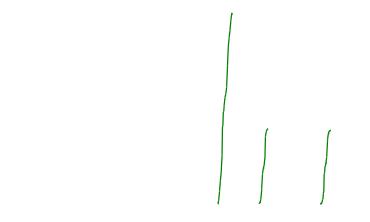

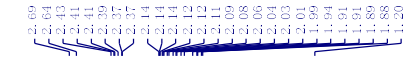
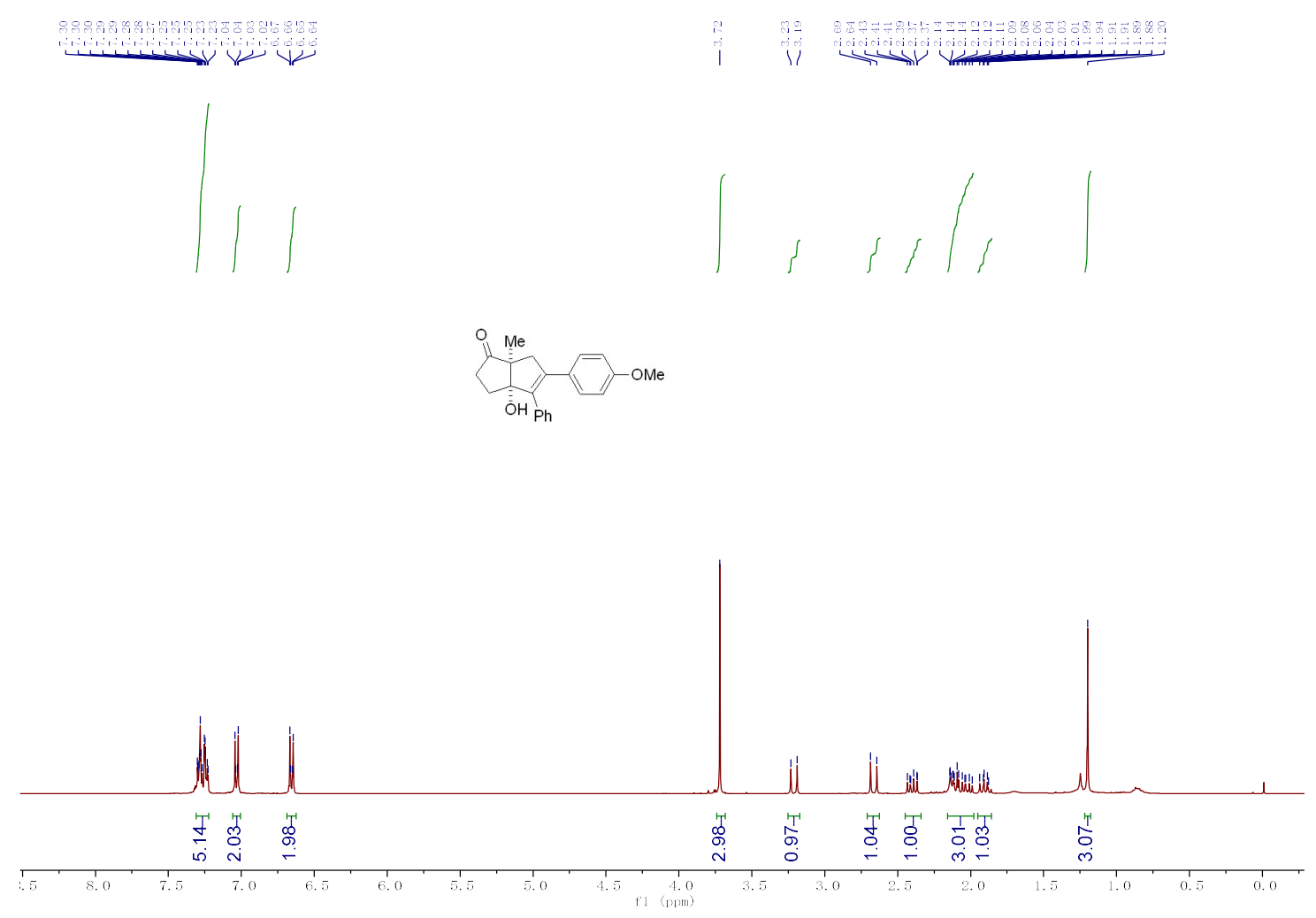

$3 \mathrm{ab}\left(101 \mathrm{MHz}\right.$ for ${ }^{13} \mathrm{C}$ NMR with $\mathrm{CDCl}_{3}$ as solvent)

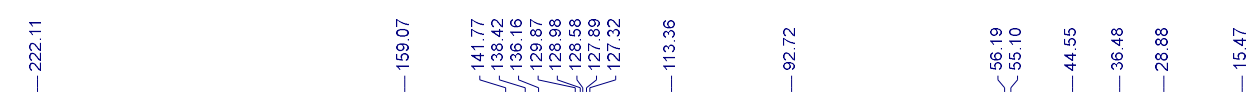

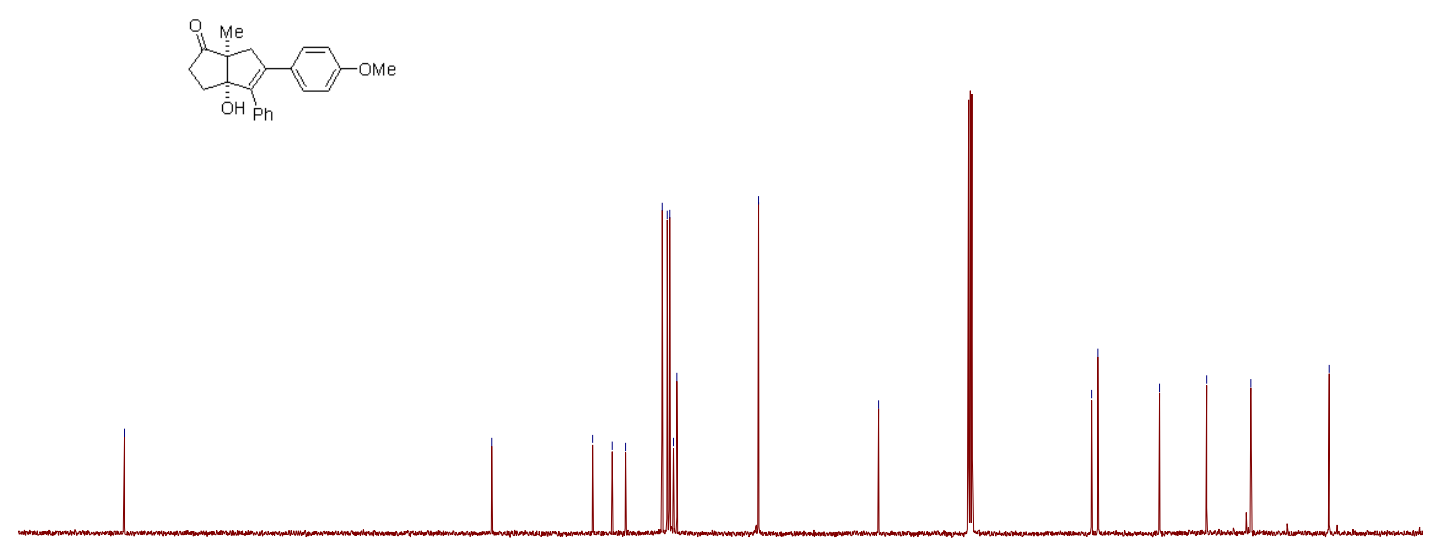


3ac (400 MHz for ${ }^{1} \mathrm{H}$ NMR with $\mathrm{CDCl}_{3}$ as solvent)
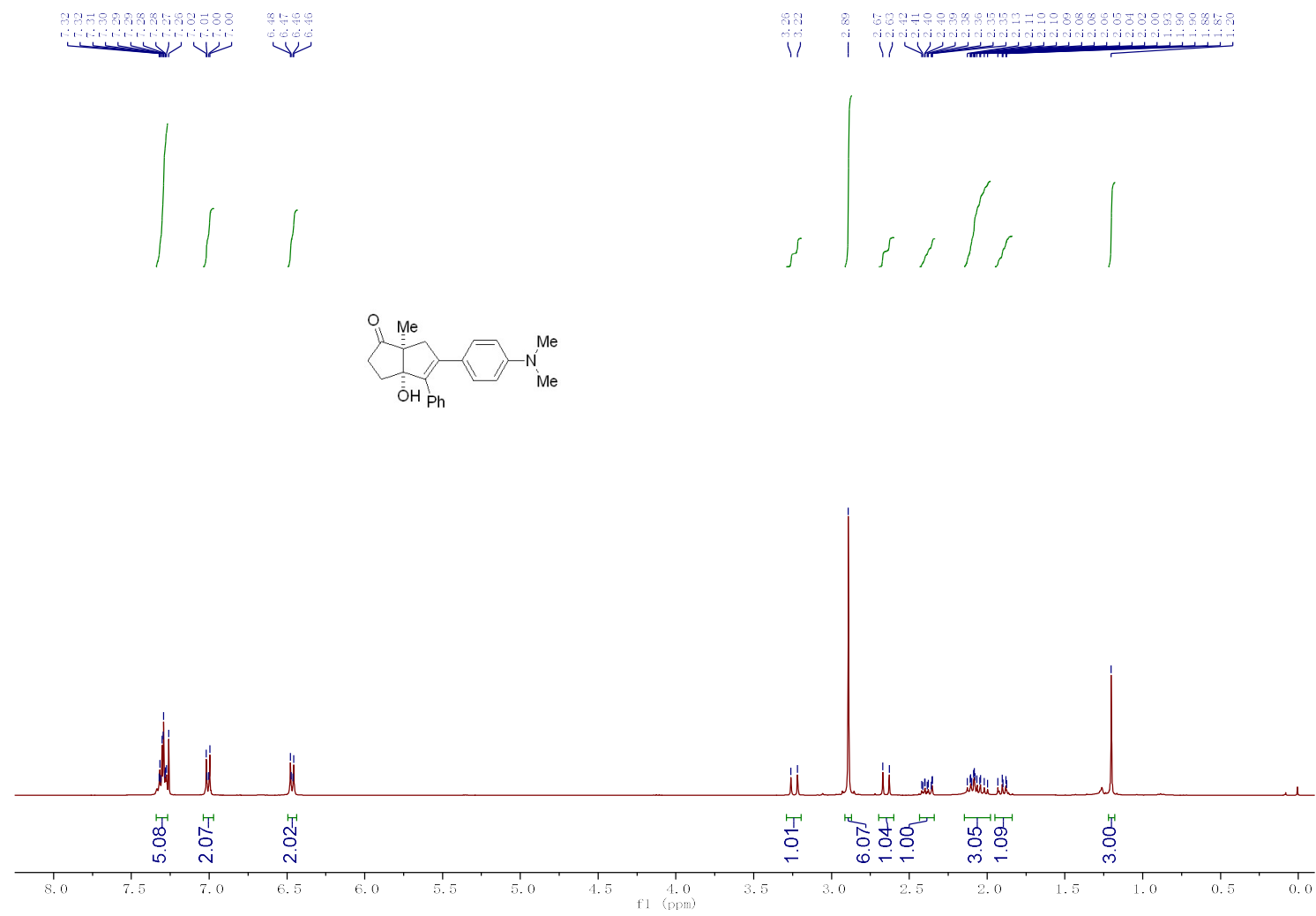

3ac (101 MHz for ${ }^{13} \mathrm{C} \mathrm{NMR}$ with $\mathrm{CDCl}_{3}$ as solvent)
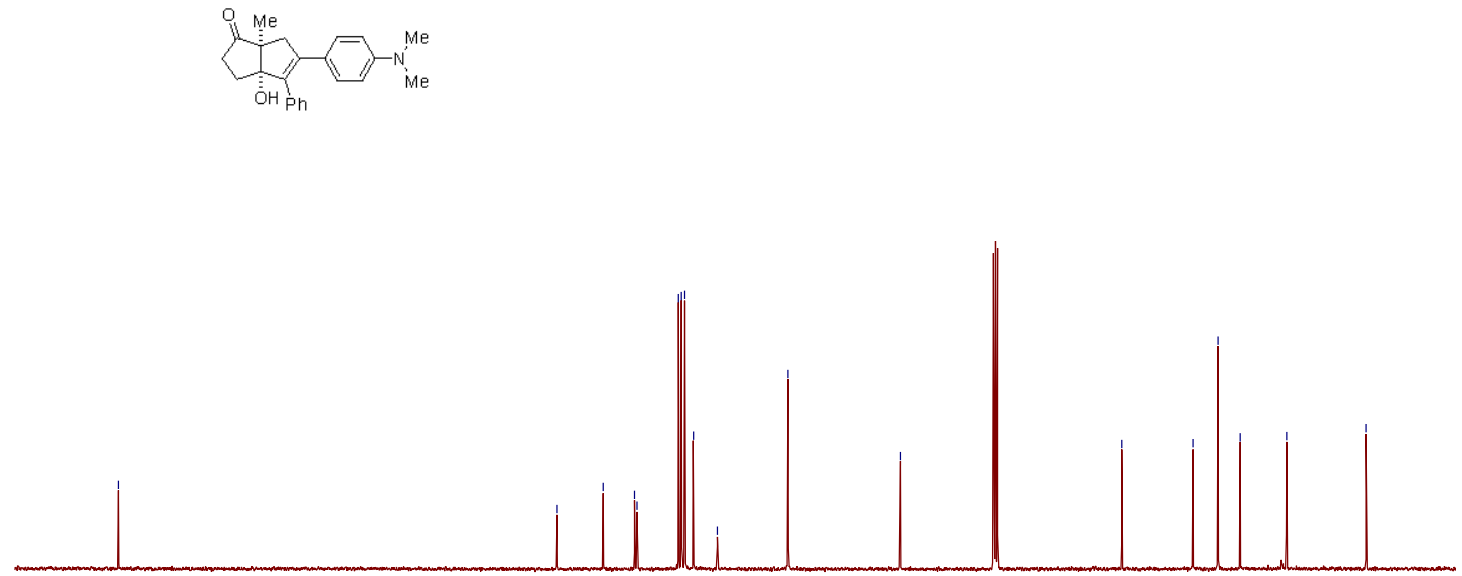
3ad (400 MHz for ${ }^{1} \mathrm{H} \mathrm{NMR}$ with $\mathrm{CDCl}_{3}$ as solvent)
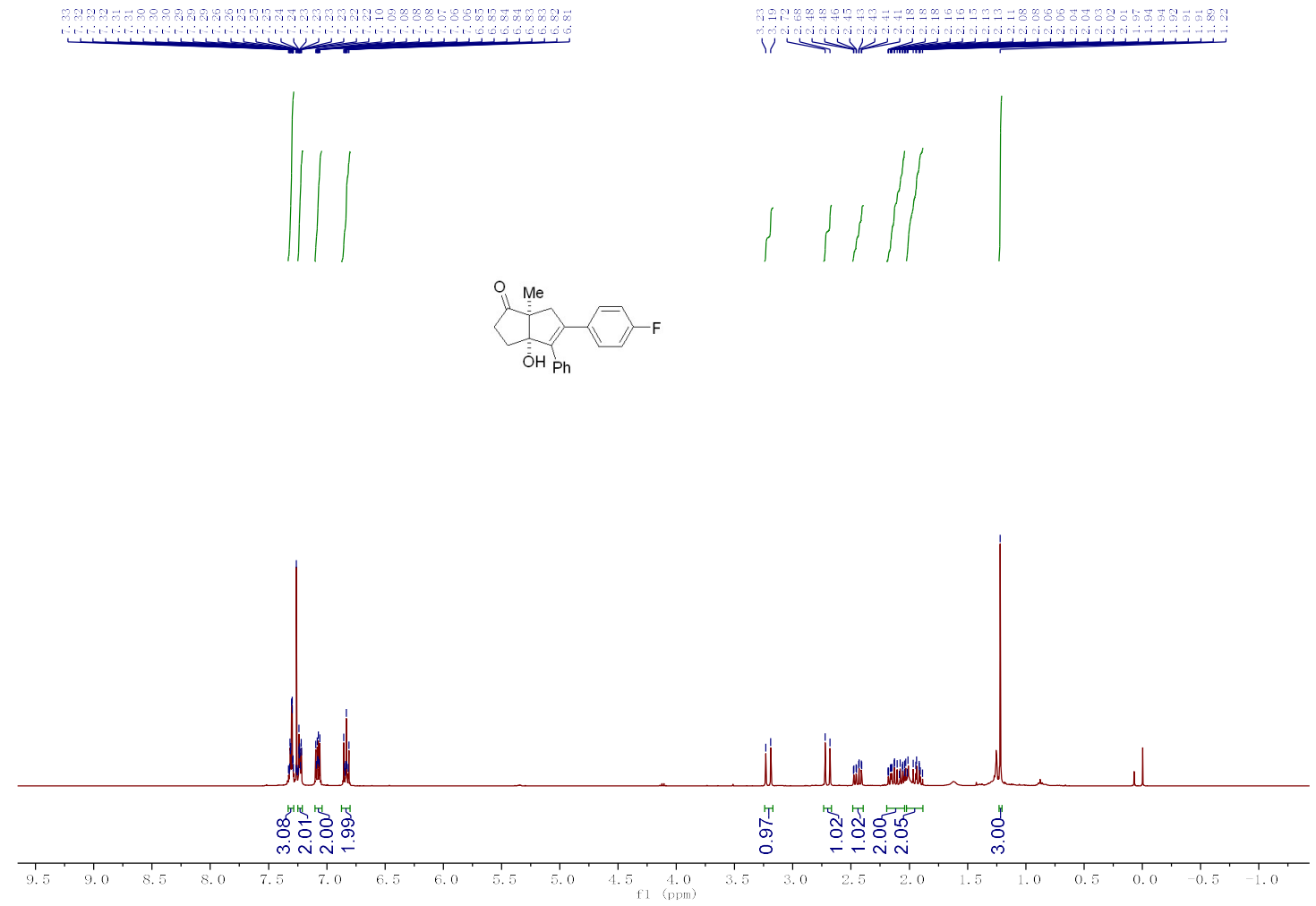

3ad (101 MHz for ${ }^{13} \mathrm{C} \mathrm{NMR}$ with $\mathrm{CDCl}_{3}$ as solvent)
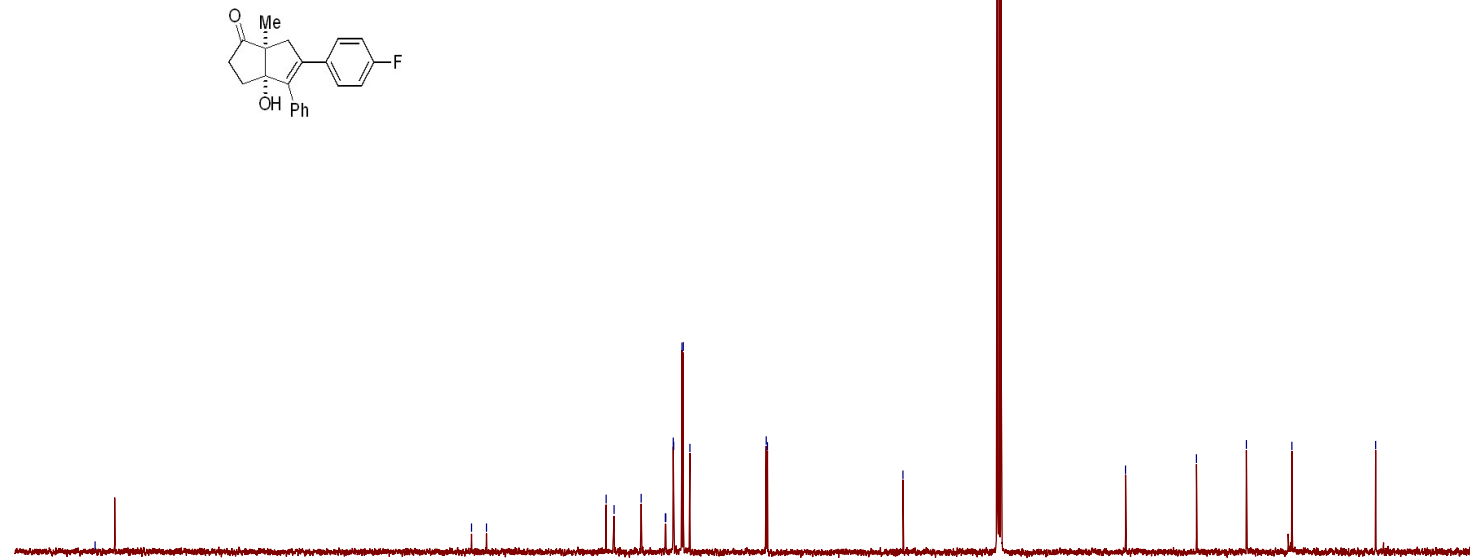
3ad (376 MHz for ${ }^{19} \mathrm{~F} \mathrm{NMR}$ with $\mathrm{CDCl}_{3}$ as solvent) liwf $1-2$
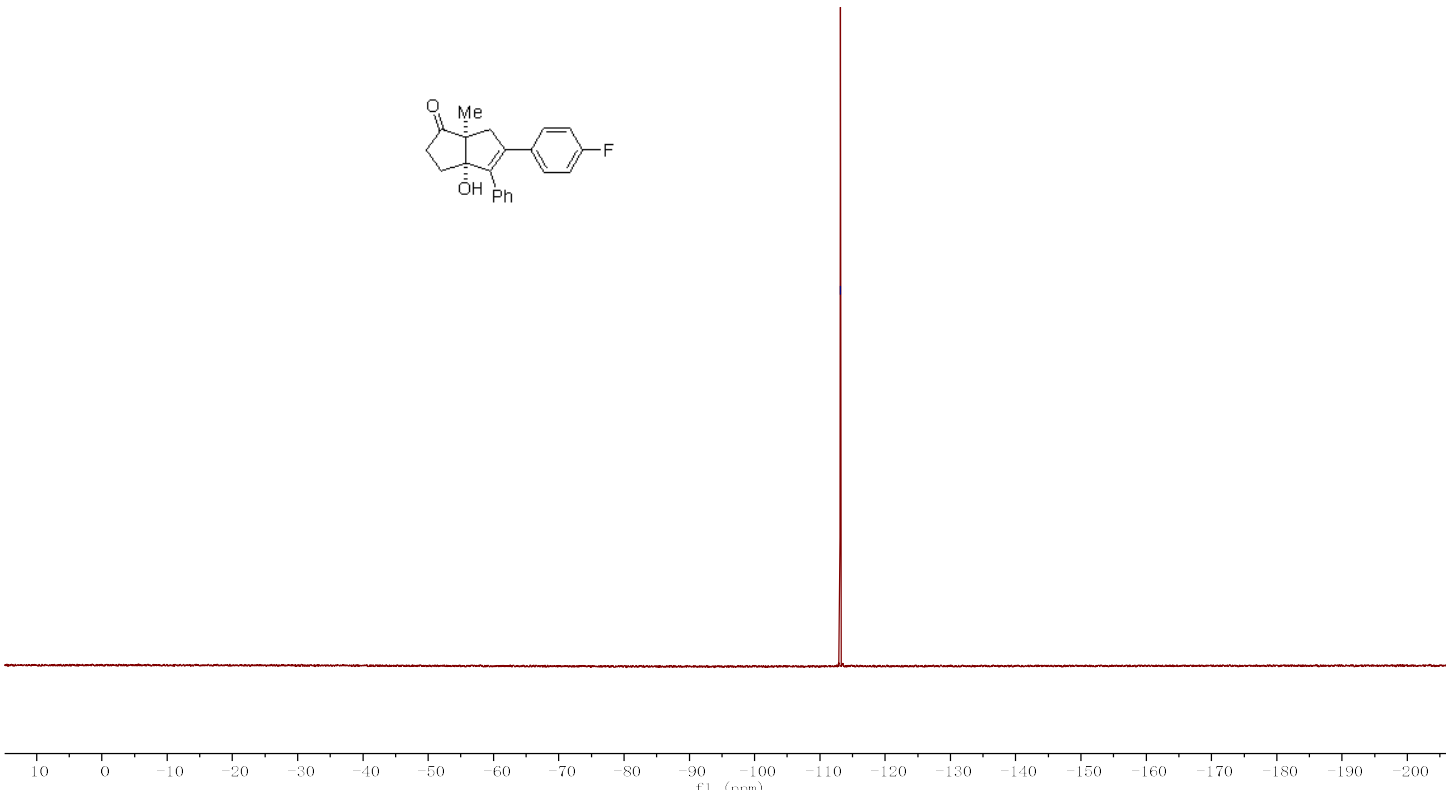
3ae (400 MHz for ${ }^{1} \mathrm{H}$ NMR with $\mathrm{CDCl}_{3}$ as solvent)

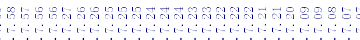

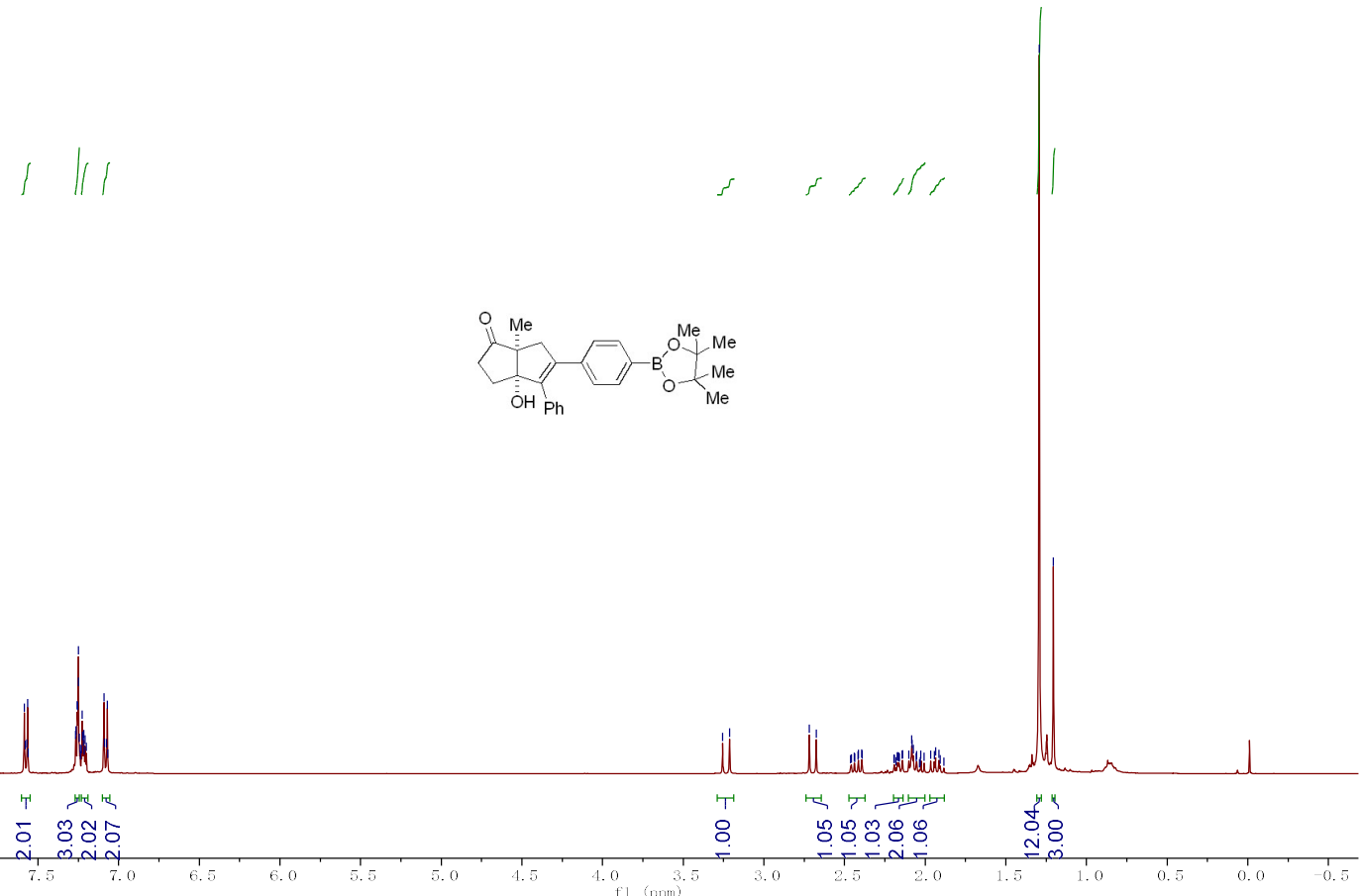

3ae (101 MHz for ${ }^{13} \mathrm{C} \mathrm{NMR}$ with $\mathrm{CDCl}_{3}$ as solvent) $\stackrel{\infty}{i}$
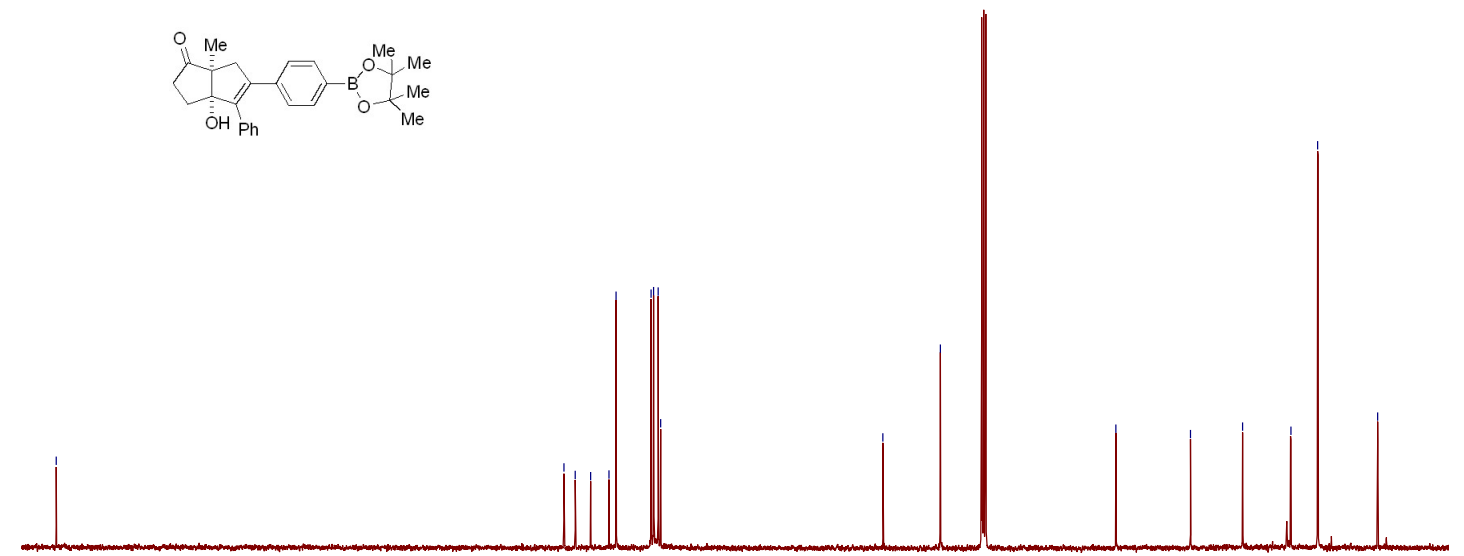
3af $\left(400 \mathrm{MHz}\right.$ for ${ }^{1} \mathrm{H} \mathrm{NMR}$ with $\mathrm{CDCl}_{3}$ as solvent)

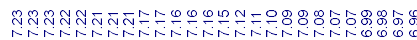

8ำ
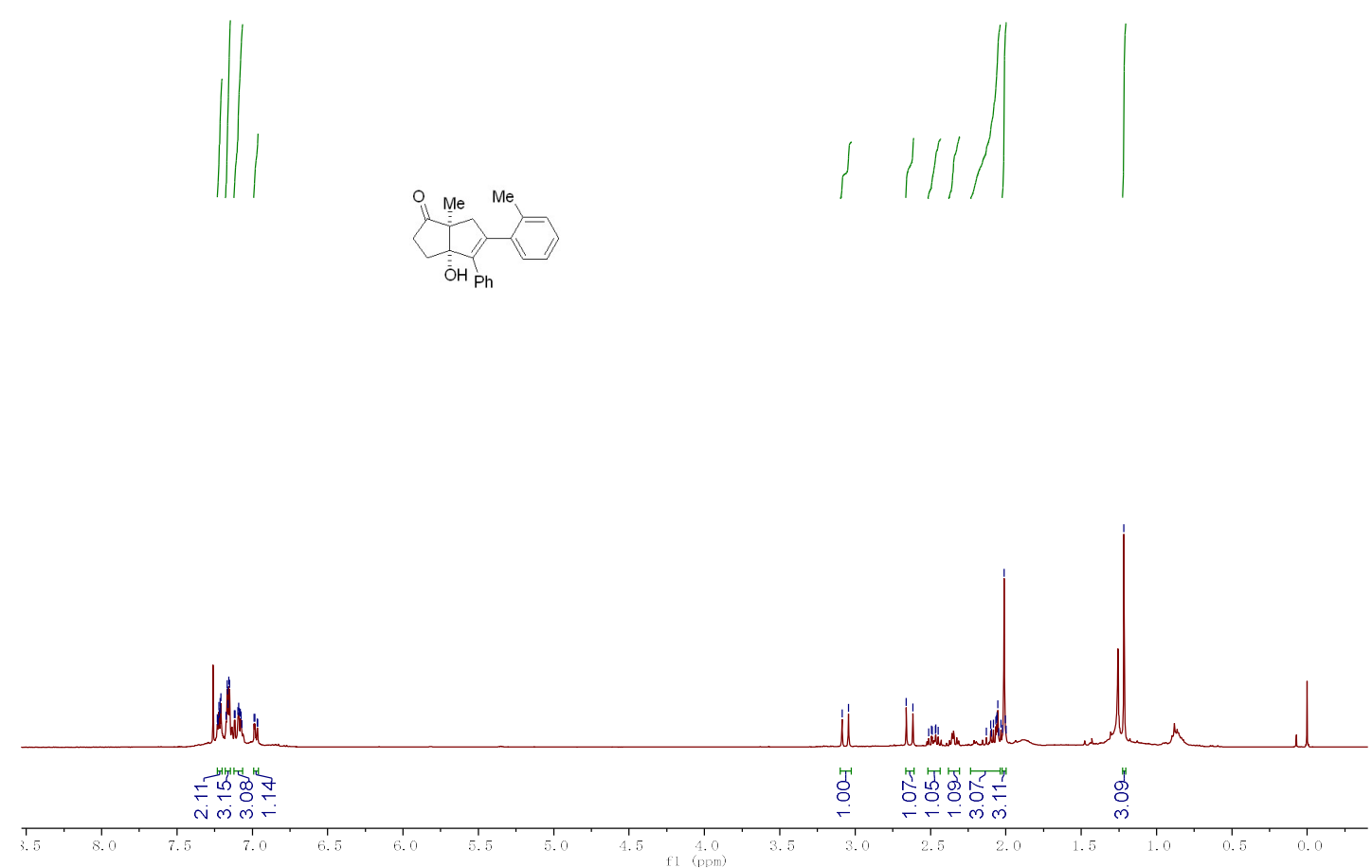

3af (101 MHz for ${ }^{13} \mathrm{C}$ NMR with $\mathrm{CDCl}_{3}$ as solvent)
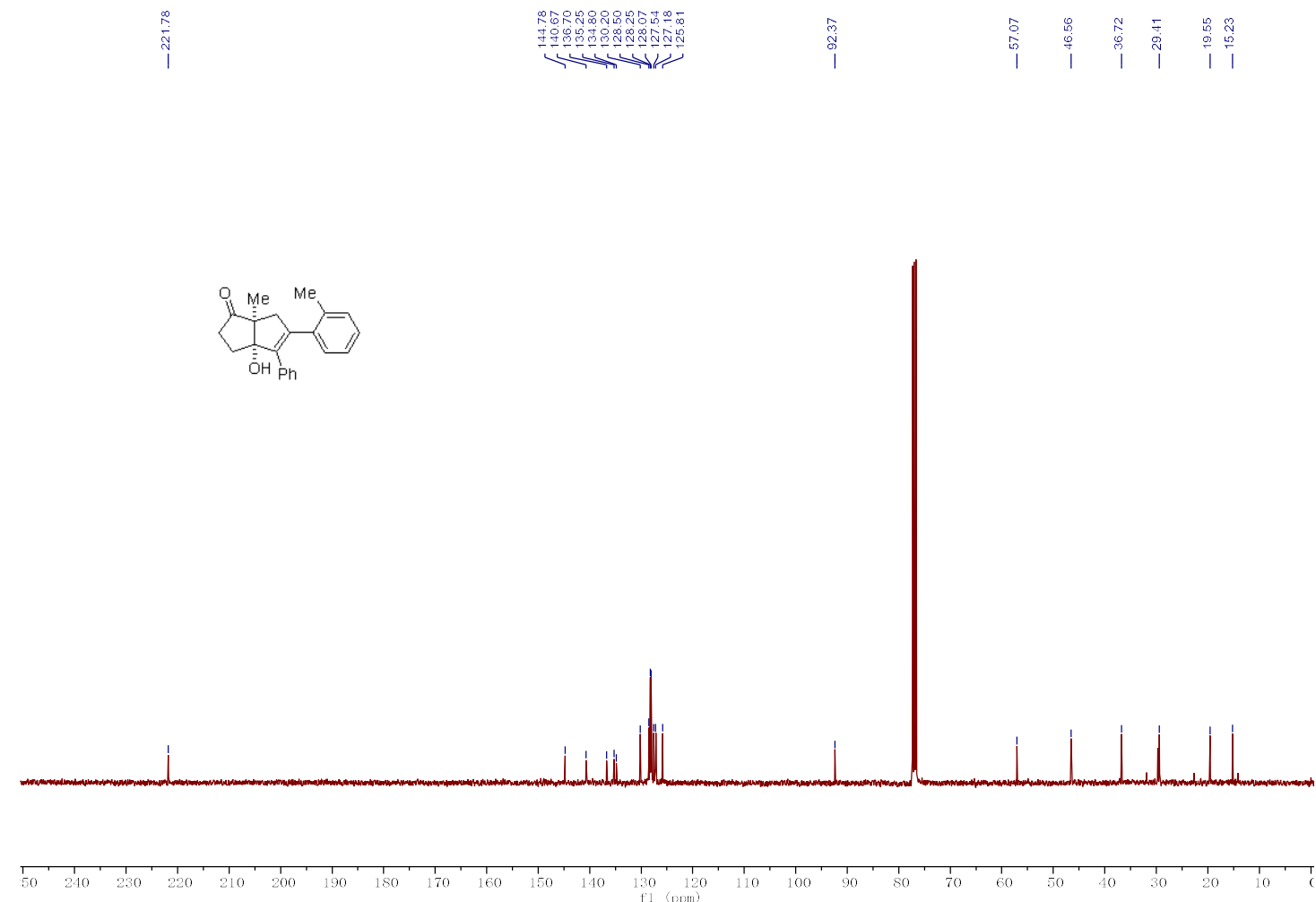
3ag (400 MHz for ${ }^{1} \mathrm{H} \mathrm{NMR}$ with $\mathrm{CDCl}_{3}$ as solvent)

BEs

每
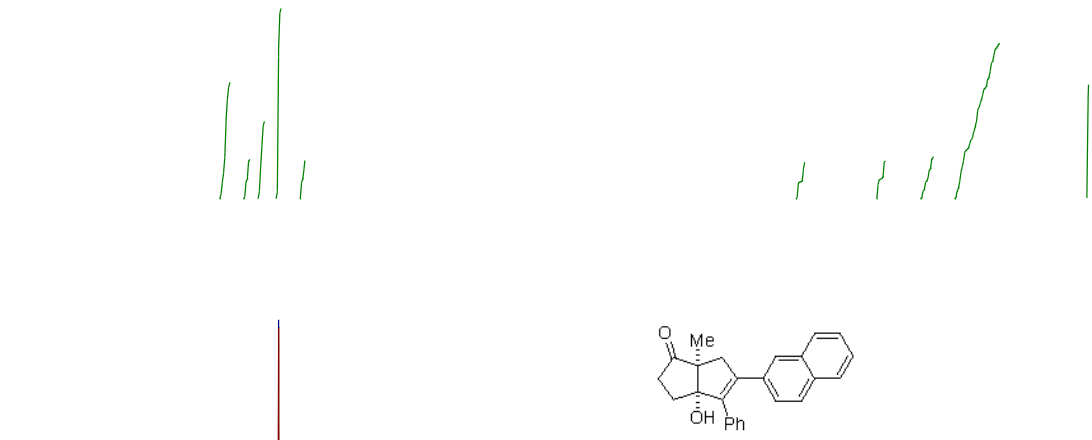

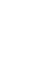

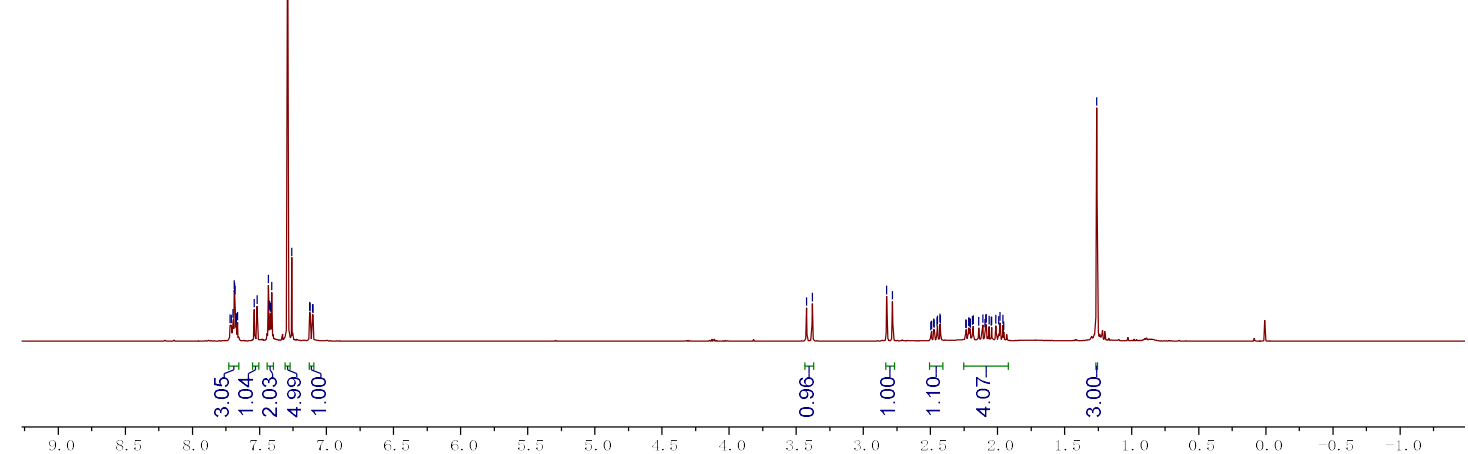

3ag (101 MHz for ${ }^{13} \mathrm{C}$ NMR with $\mathrm{CDCl}_{3}$ as solvent)
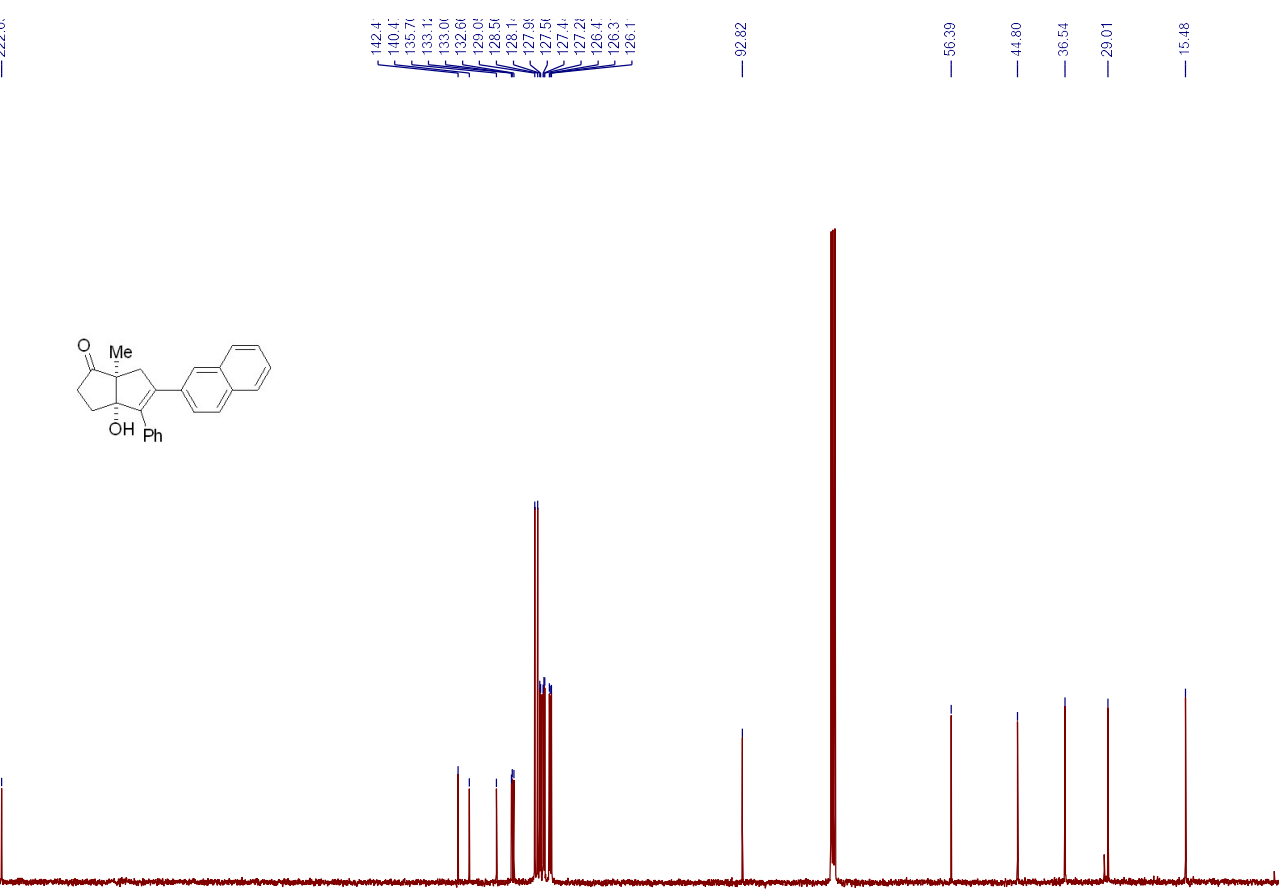

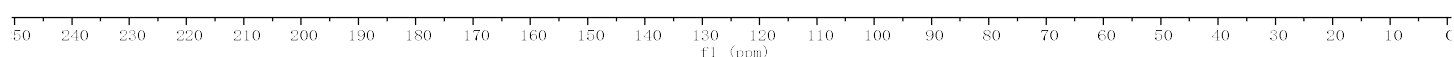


3ah (400 MHz for ${ }^{1} \mathrm{H}$ NMR with $\mathrm{CDCl}_{3}$ as solvent)

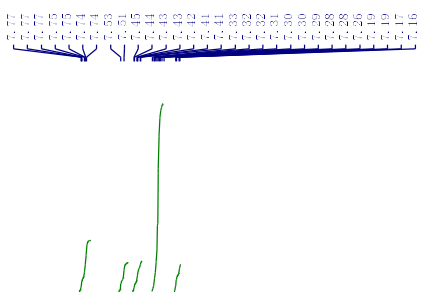

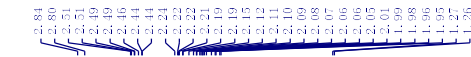
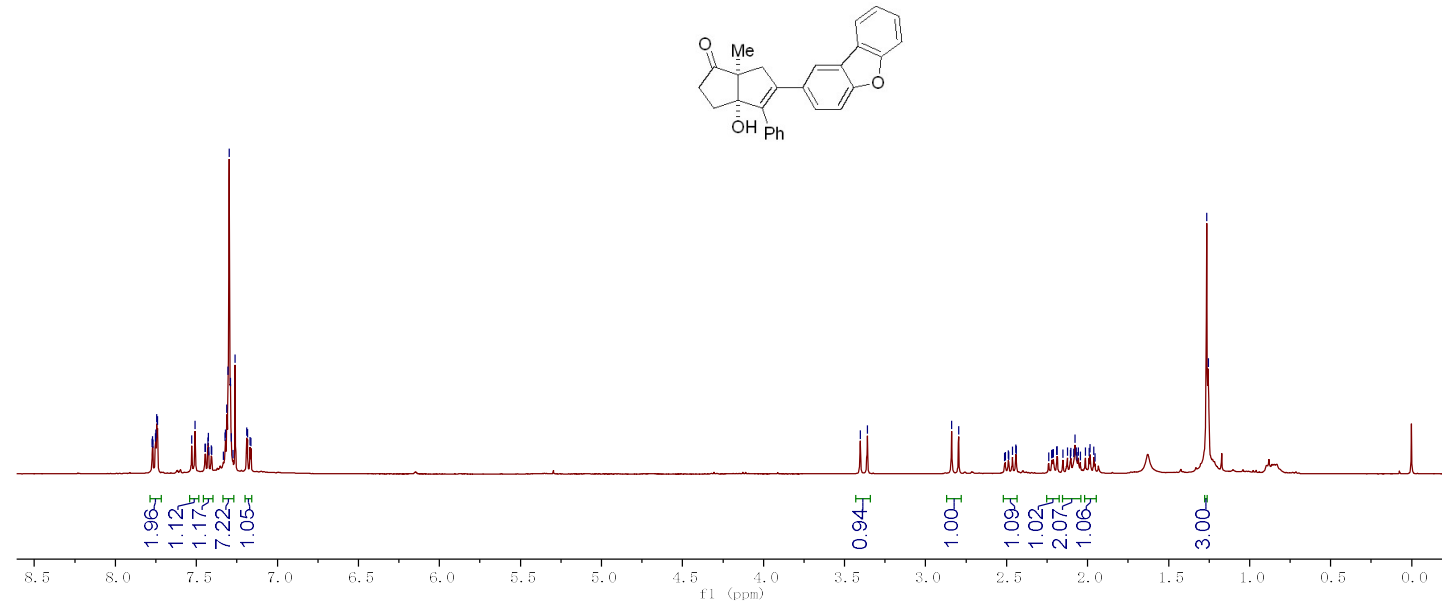

3ah (101 MHz for ${ }^{13} \mathrm{C}$ NMR with $\mathrm{CDCl}_{3}$ as solvent)
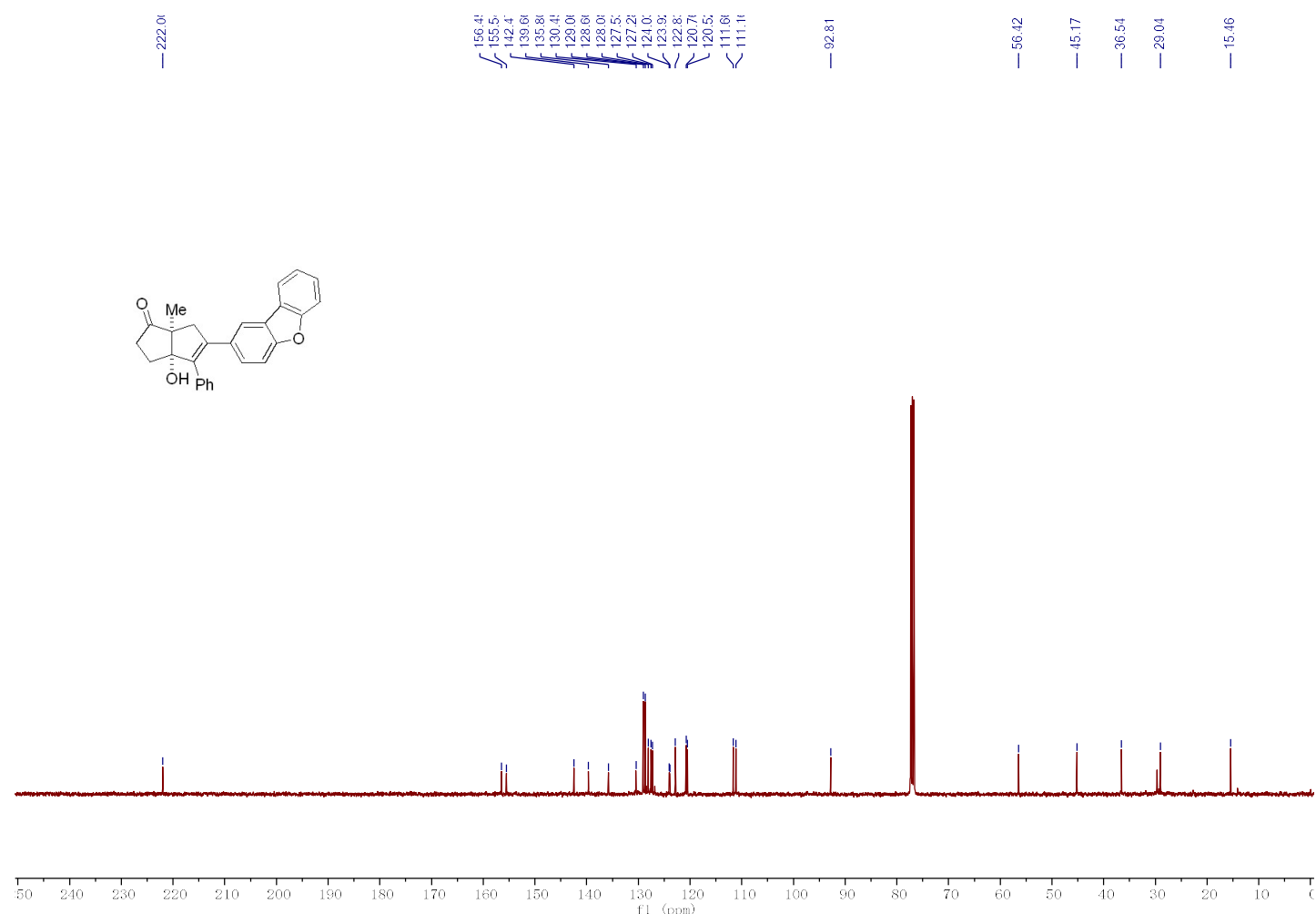
3ai (400 MHz for ${ }^{1} \mathrm{H} ~ N M R$ with $\mathrm{CDCl}_{3}$ as solvent)

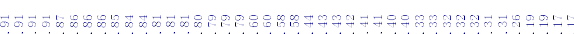

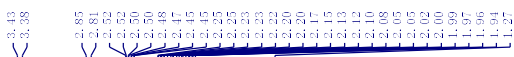

||| |||
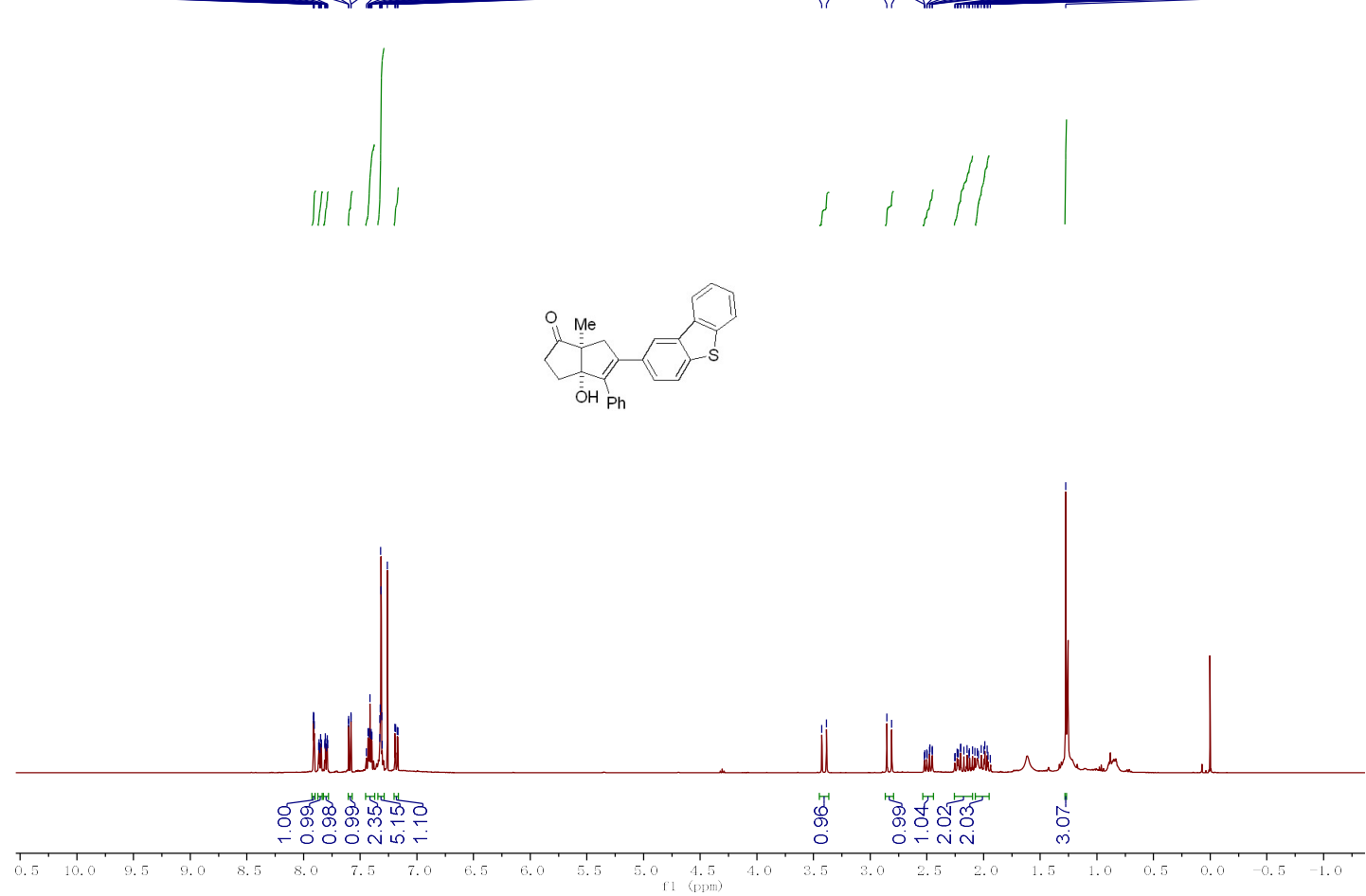

3ai (101 MHz for ${ }^{13} \mathrm{C}$ NMR with $\mathrm{CDCl}_{3}$ as solvent)

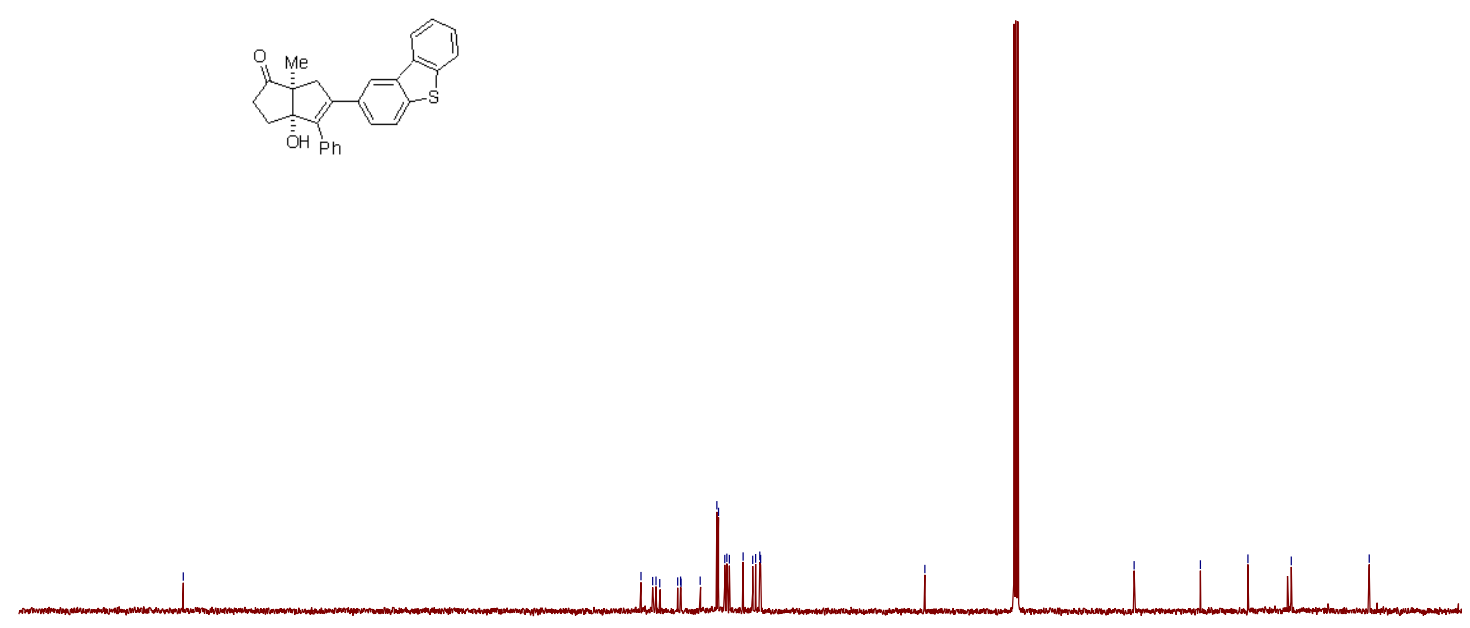

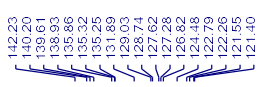

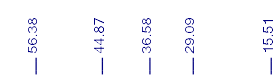


3aj (400 MHz for ${ }^{1} \mathrm{H}$ NMR with $\mathrm{CDCl}_{3}$ as solvent)

tg8

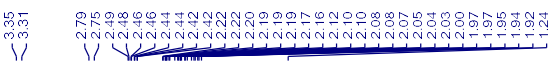
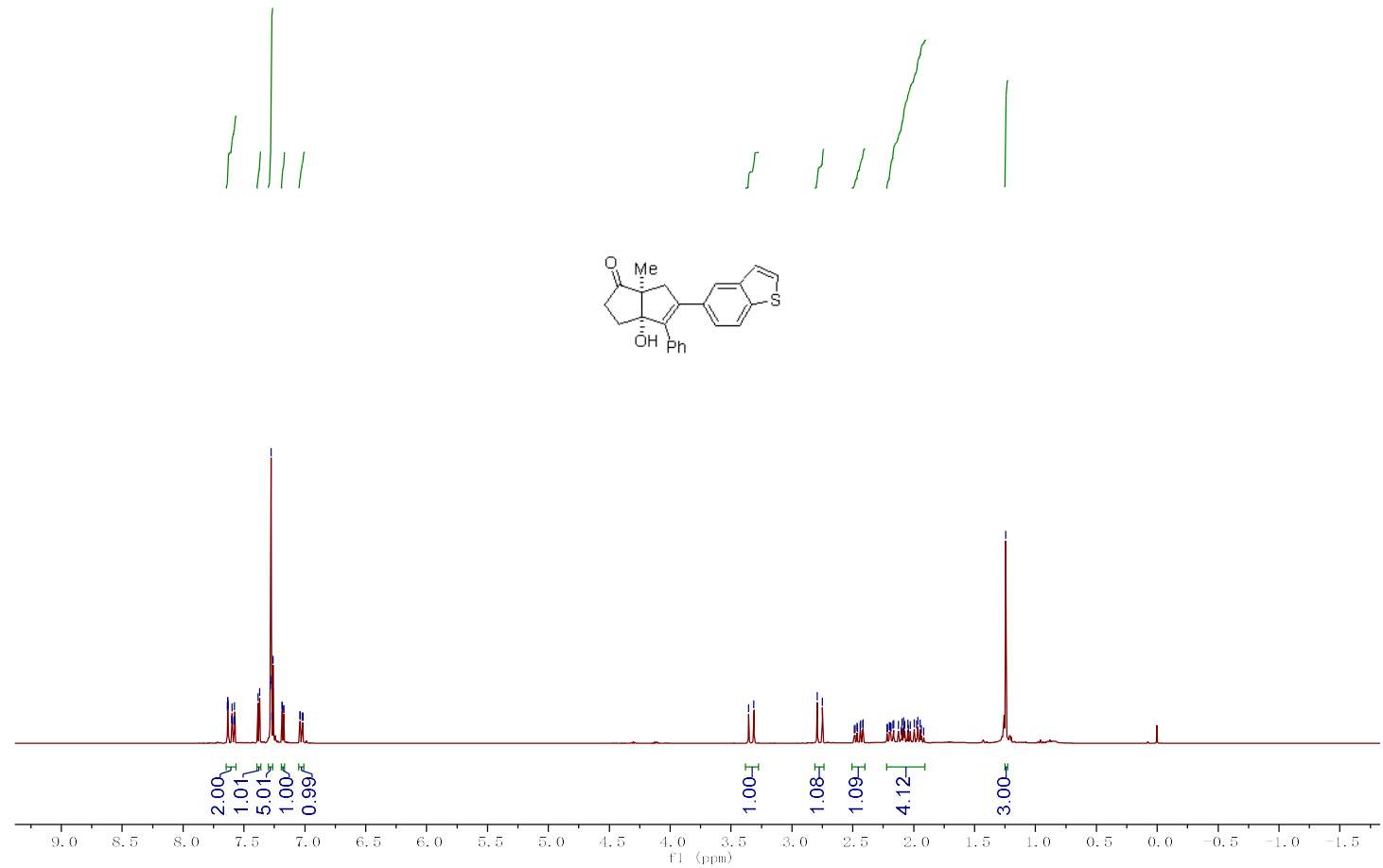

3aj (101 MHz for ${ }^{13} \mathrm{C}$ NMR with $\mathrm{CDCl}_{3}$ as solvent)

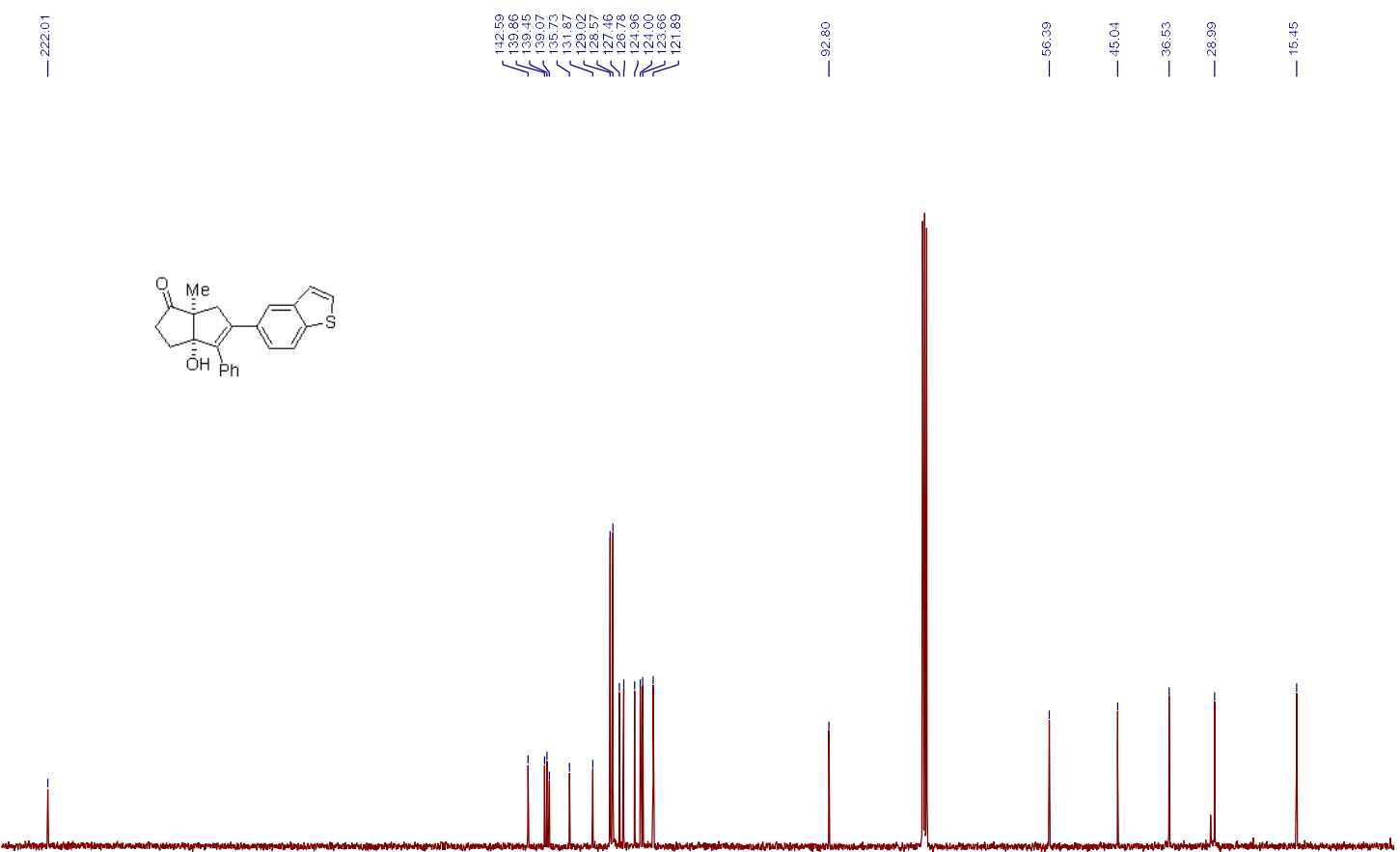


3ak (400 MHz for ${ }^{1} \mathrm{H}$ NMR with $\mathrm{CDCl}_{3}$ as solvent)

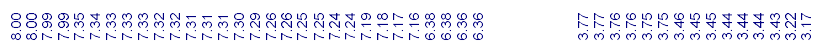
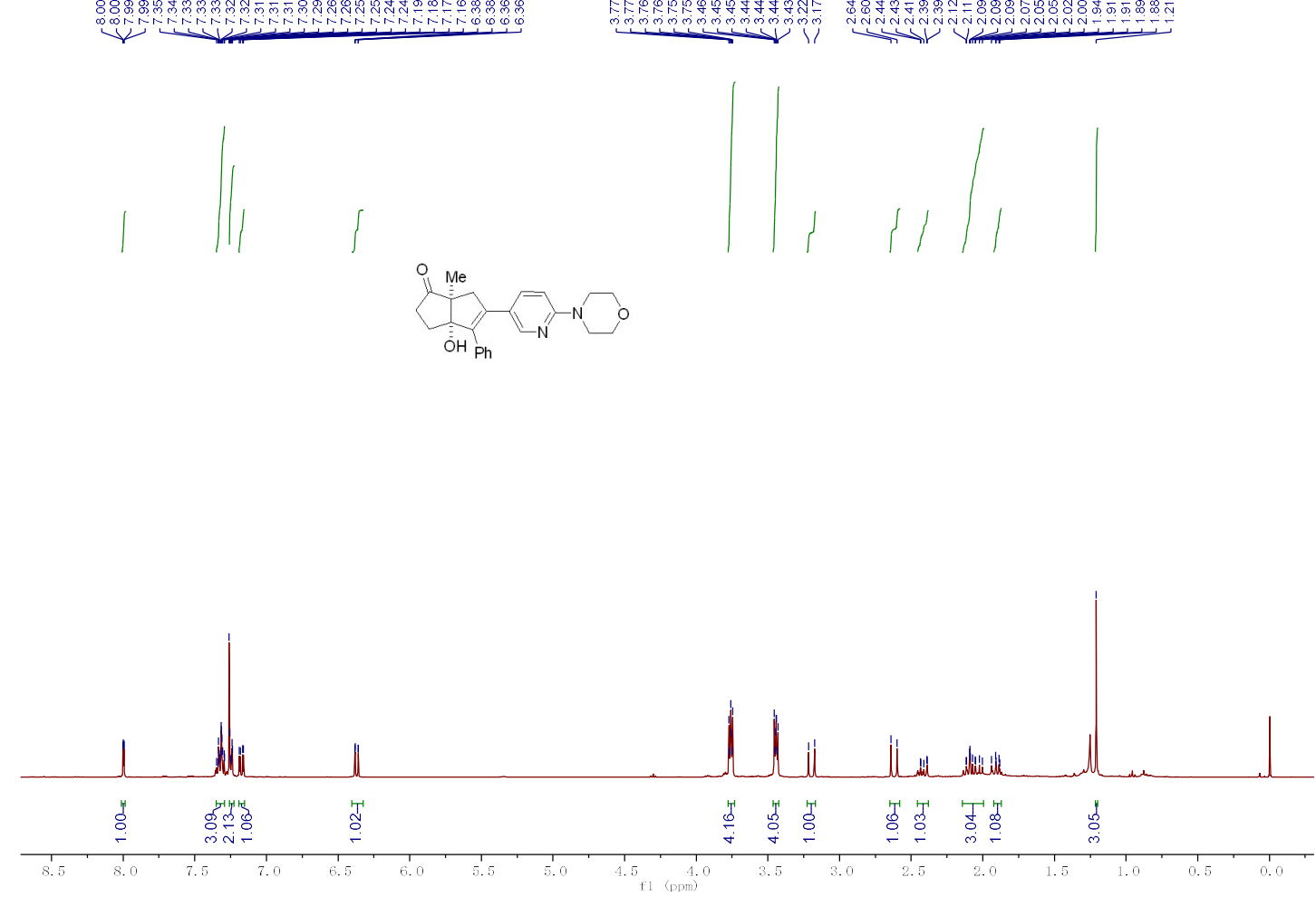

3ak (101 MHz for ${ }^{13} \mathrm{C}$ NMR with $\mathrm{CDCl}_{3}$ as solvent)

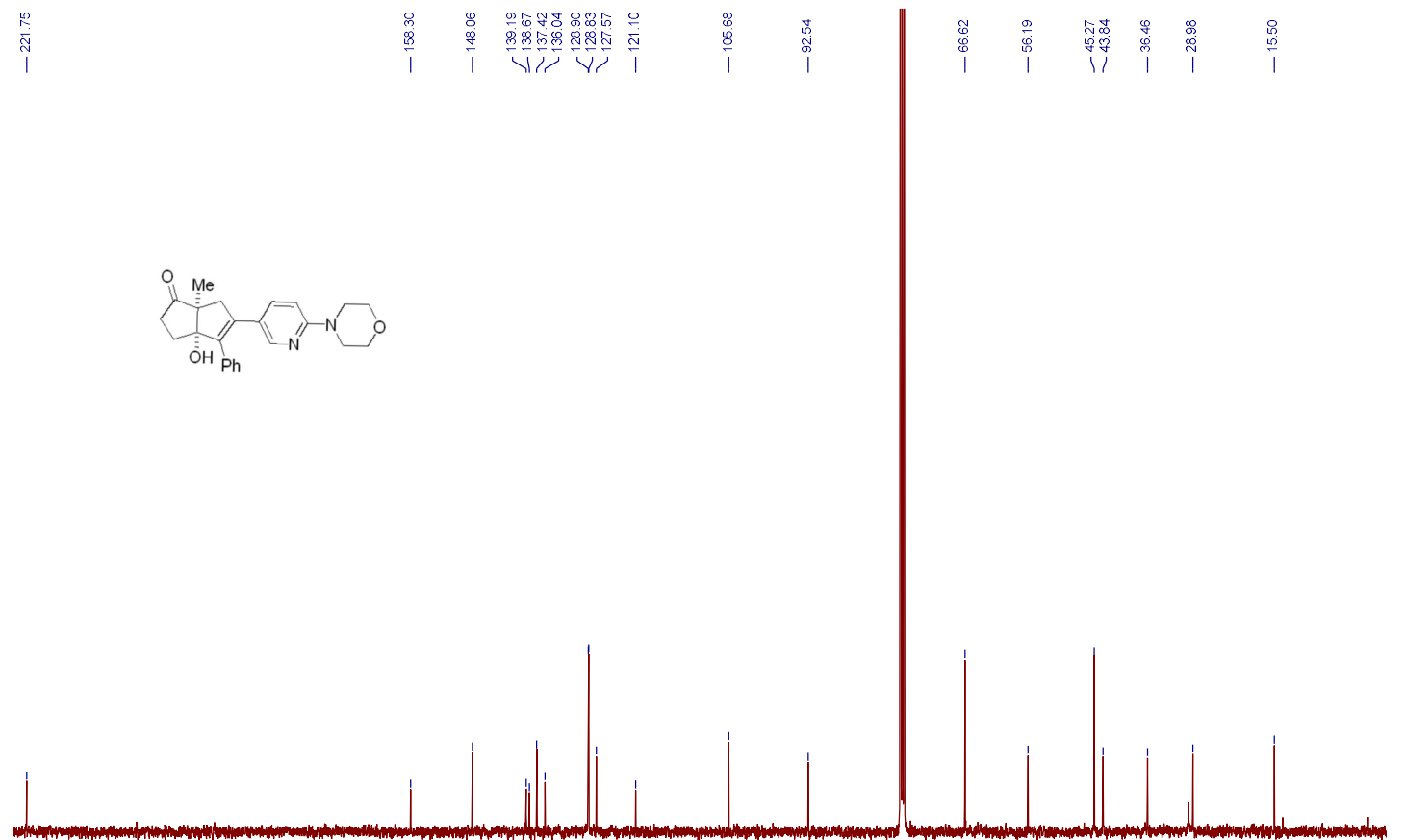


3al (400 MHz for ${ }^{1} \mathrm{H}$ NMR with $\mathrm{CDCl}_{3}$ as solvent)

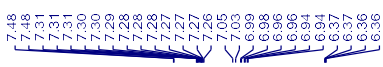

8.
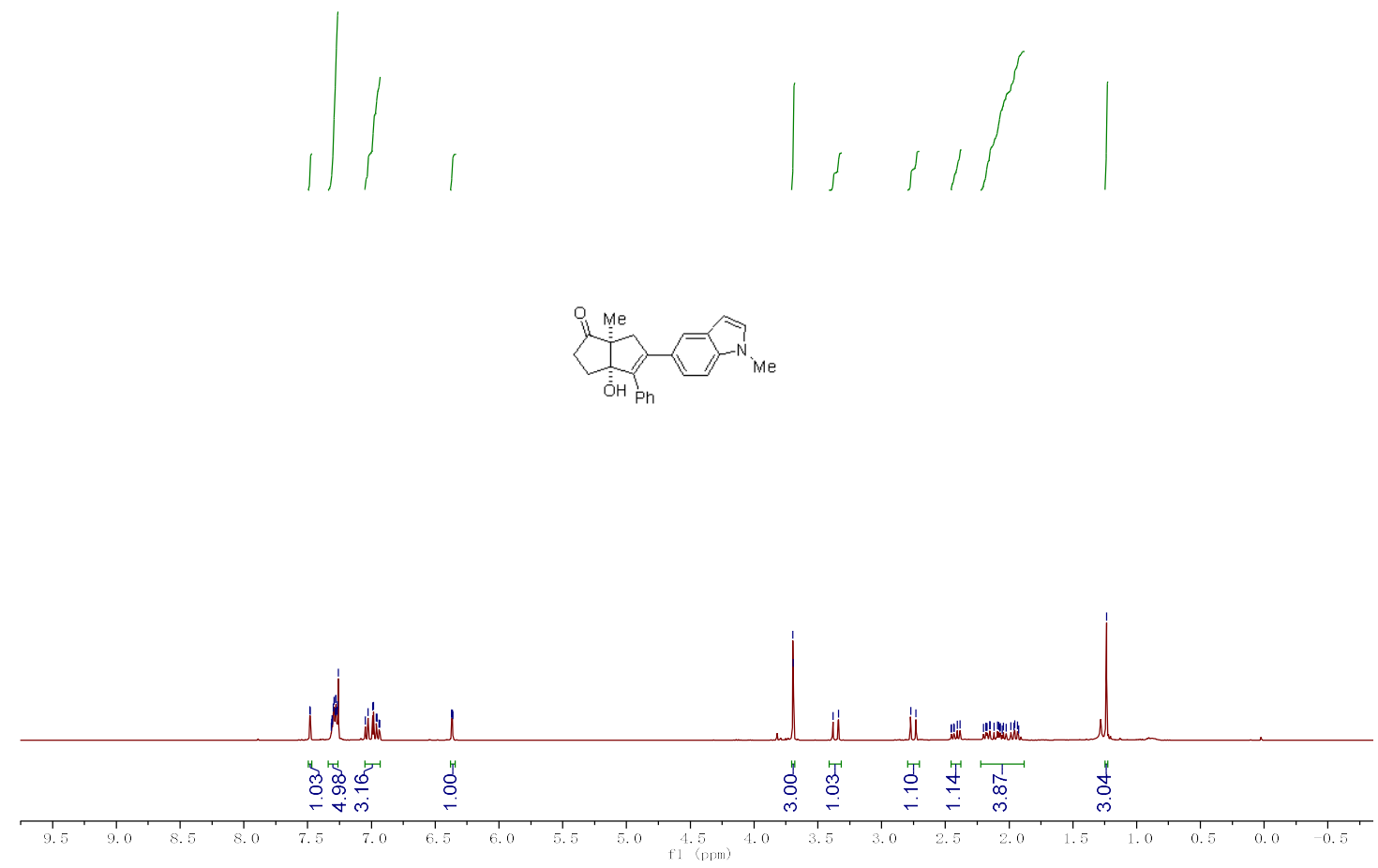

3al (101 MHz for ${ }^{13} \mathrm{C}$ NMR with $\mathrm{CDCl}_{3}$ as solvent)
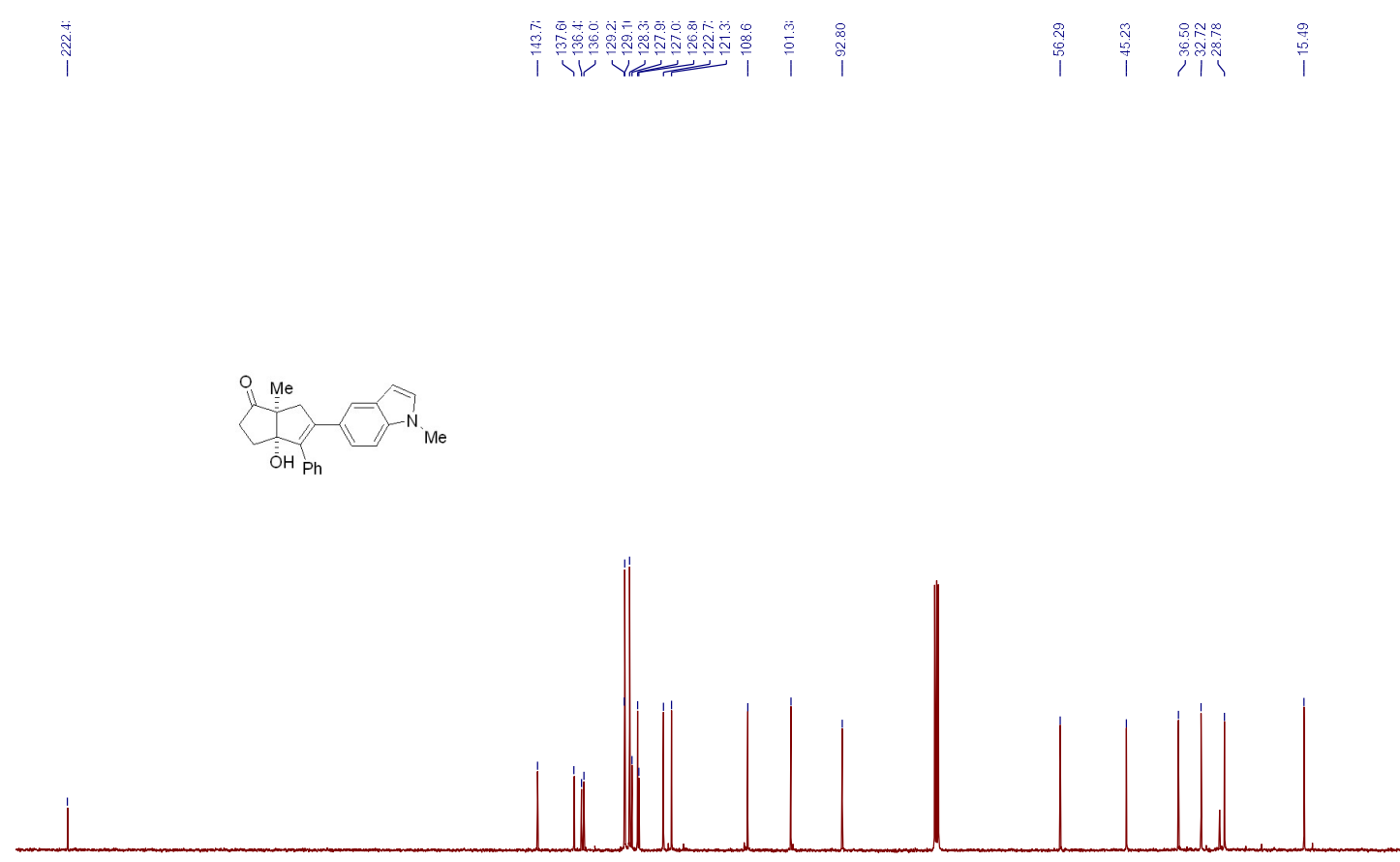
3am $\left(600 \mathrm{MHz}\right.$ for ${ }^{1} \mathrm{H}$ NMR with $\mathrm{CDCl}_{3}$ as solvent)

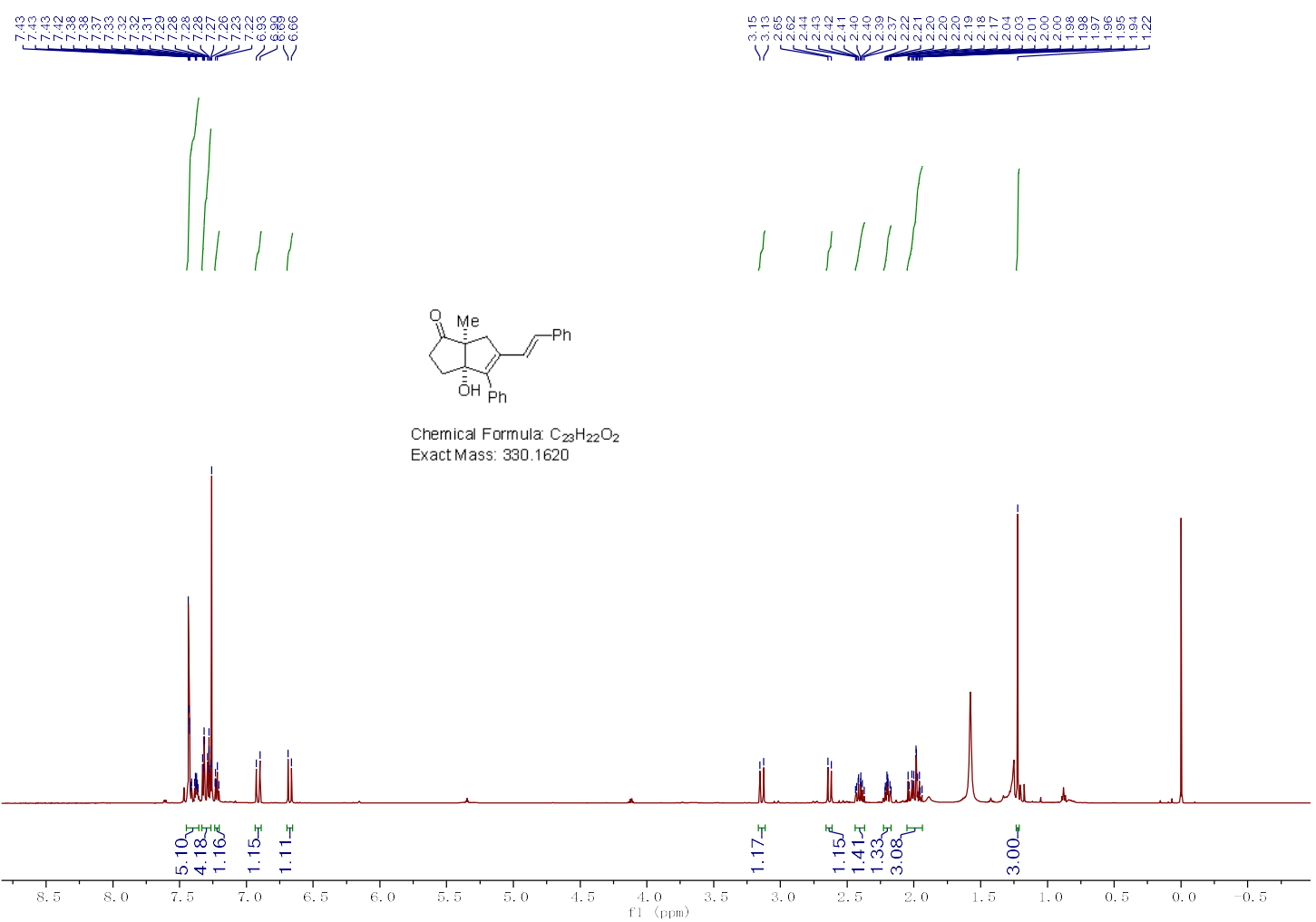

3am (151 MHz for ${ }^{13} \mathrm{C}$ NMR with $\mathrm{CDCl}_{3}$ as solvent)

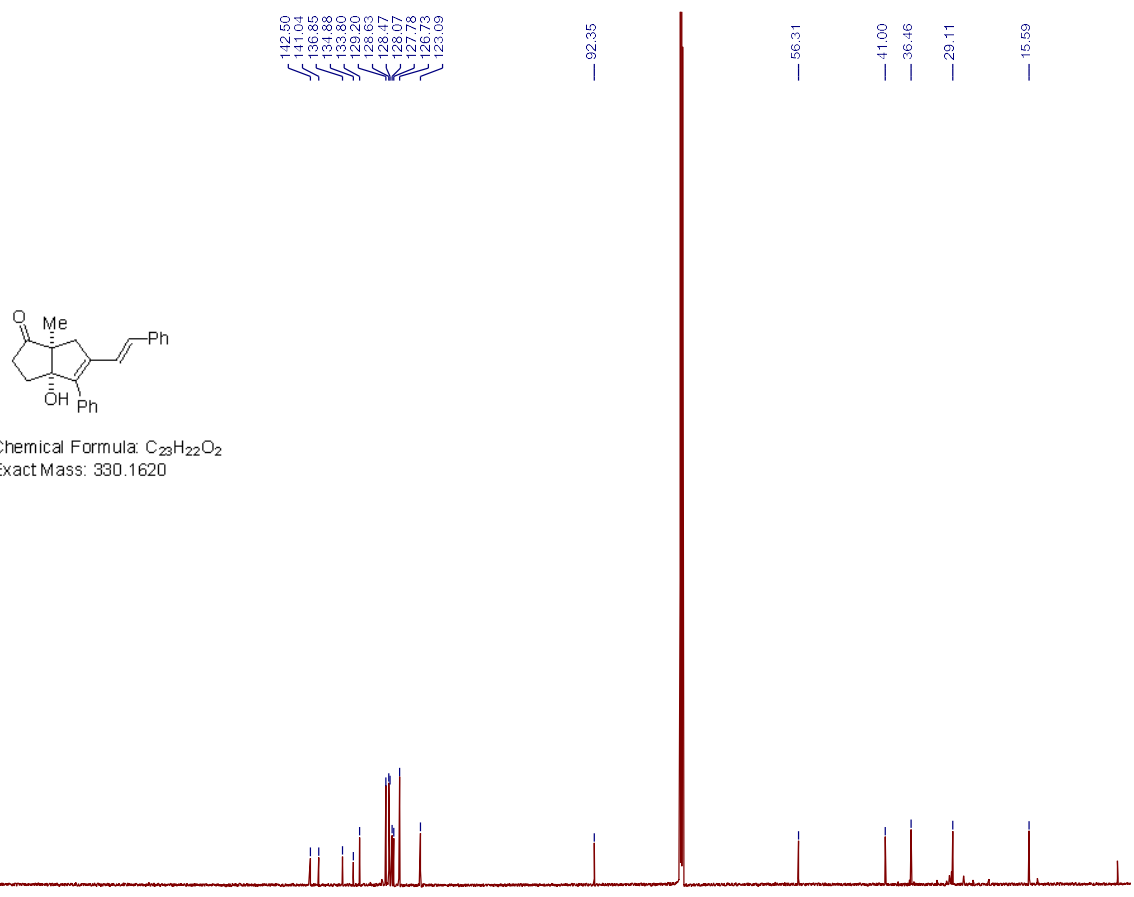

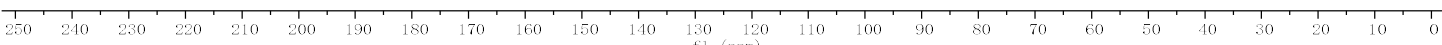


3ba (400 MHz for ${ }^{1} \mathrm{H} \mathrm{NMR}$ with $\mathrm{CDCl}_{3}$ as solvent)

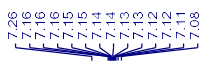

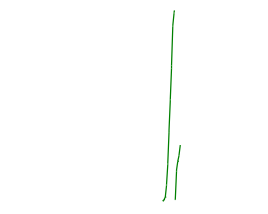

85 ำ
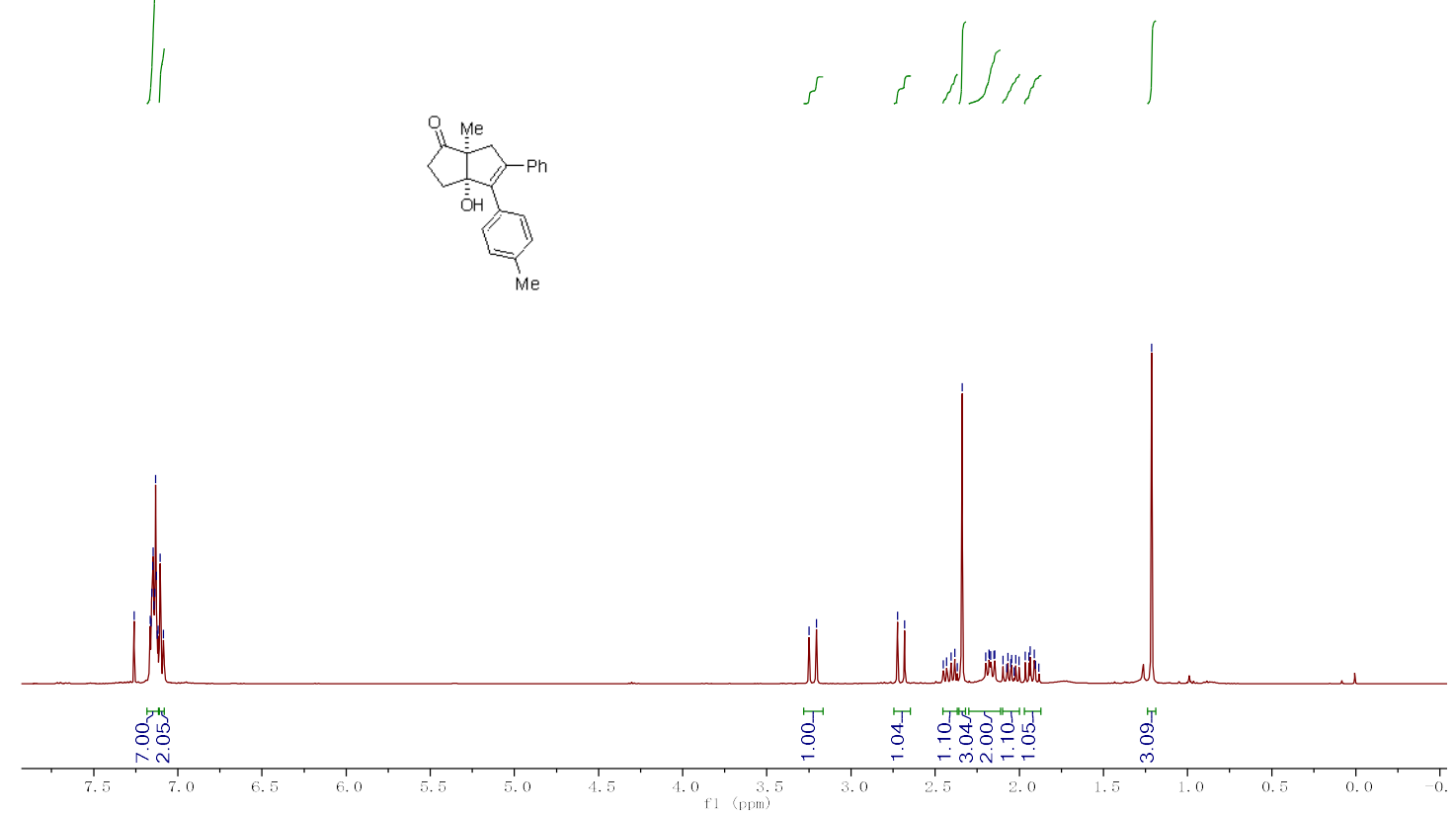

3ba (101 MHz for ${ }^{13} \mathrm{C} \mathrm{NMR}$ with $\mathrm{CDCl}_{3}$ as solvent)

$$
\text { ป }
$$
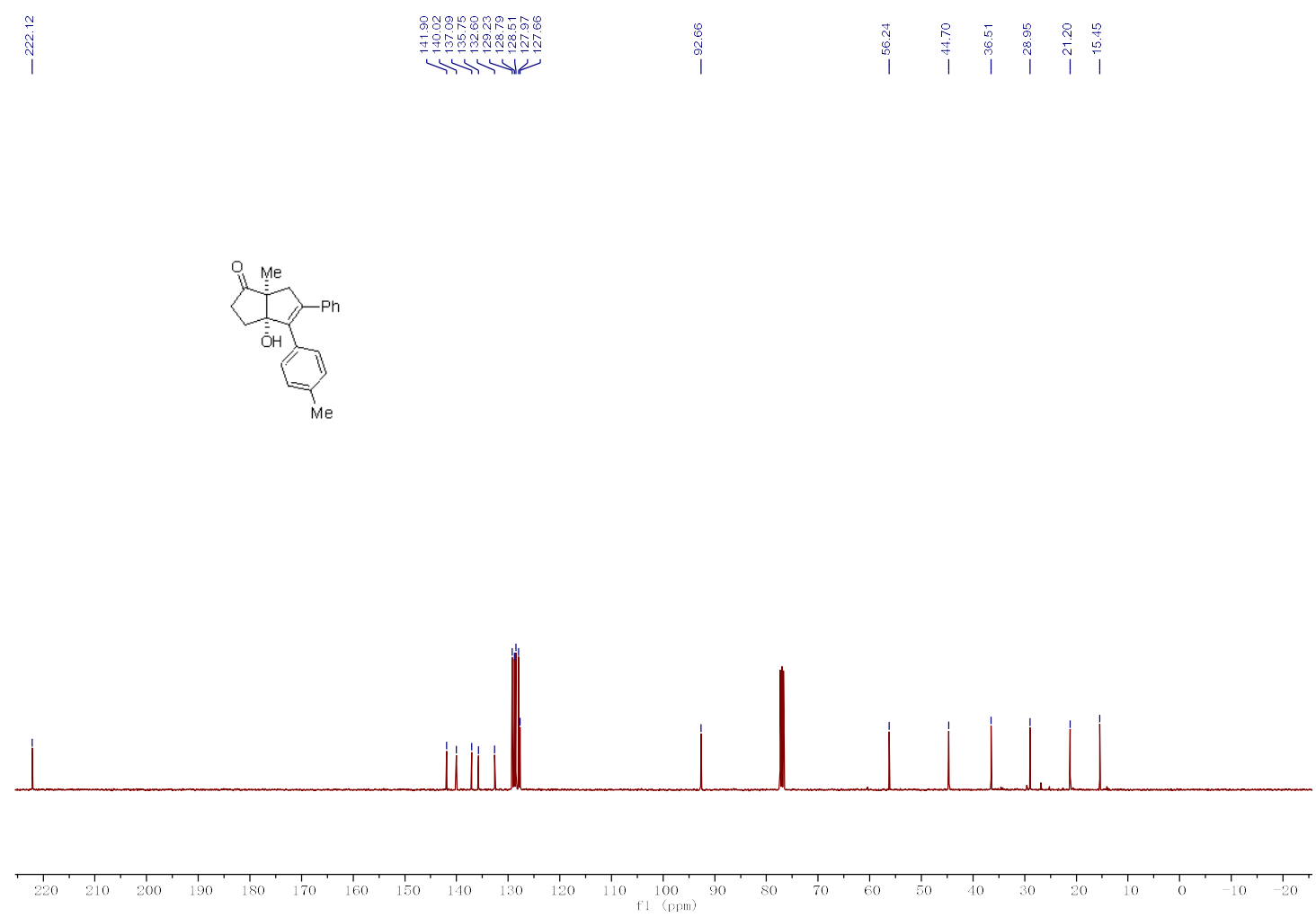
3ca (400 MHz for ${ }^{1} \mathrm{H}$ NMR with $\mathrm{CDCl}_{3}$ as solvent)

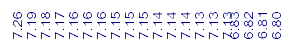
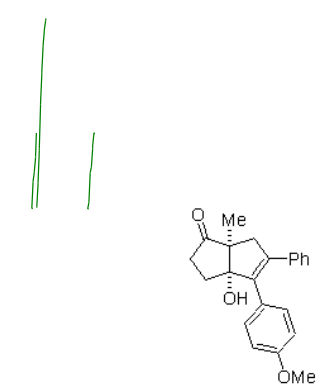

1
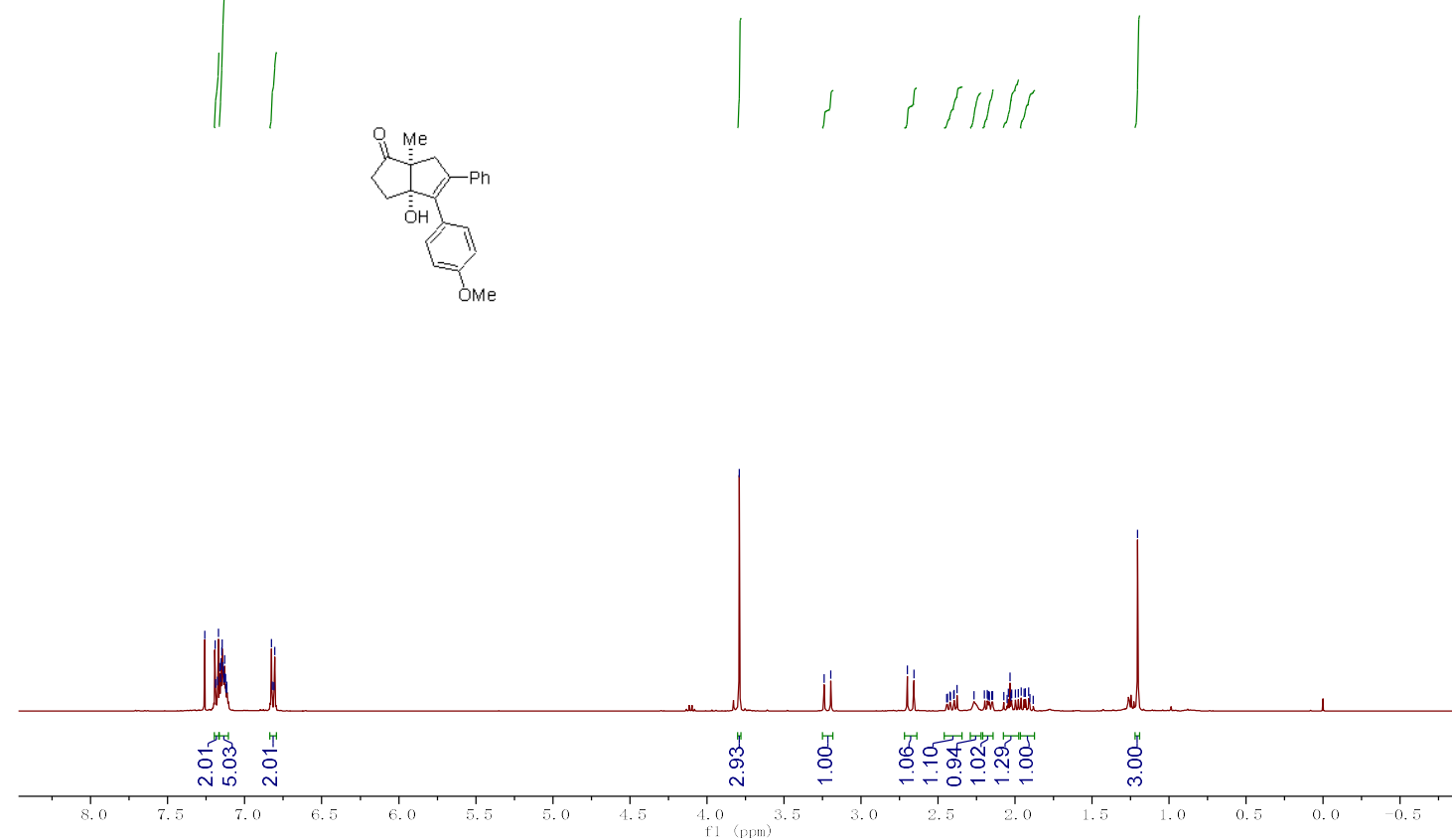

3ca (101 MHz for ${ }^{13} \mathrm{C}$ NMR with $\mathrm{CDCl}_{3}$ as solvent)

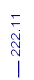

$11 \underbrace{1080}$
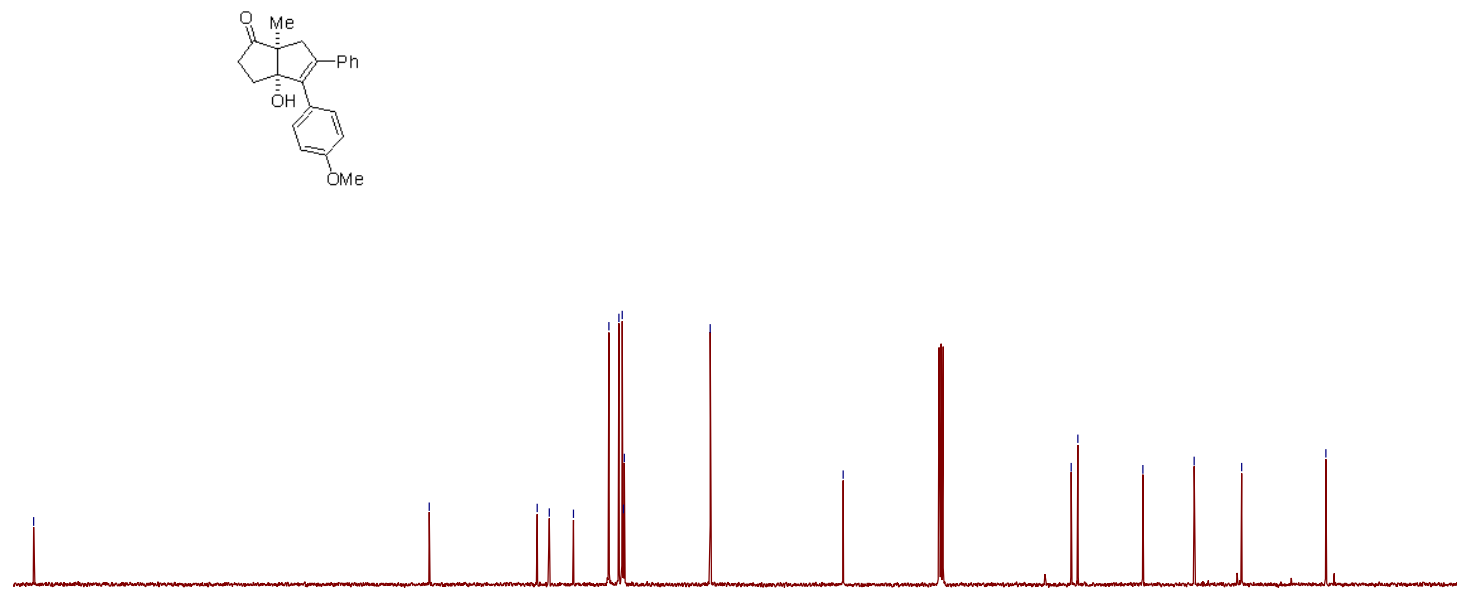
3da (400 MHz for ${ }^{1} \mathrm{H} \mathrm{NMR}$ with $\mathrm{CDCl}_{3}$ as solvent)

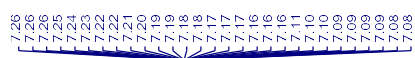

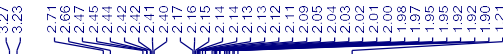
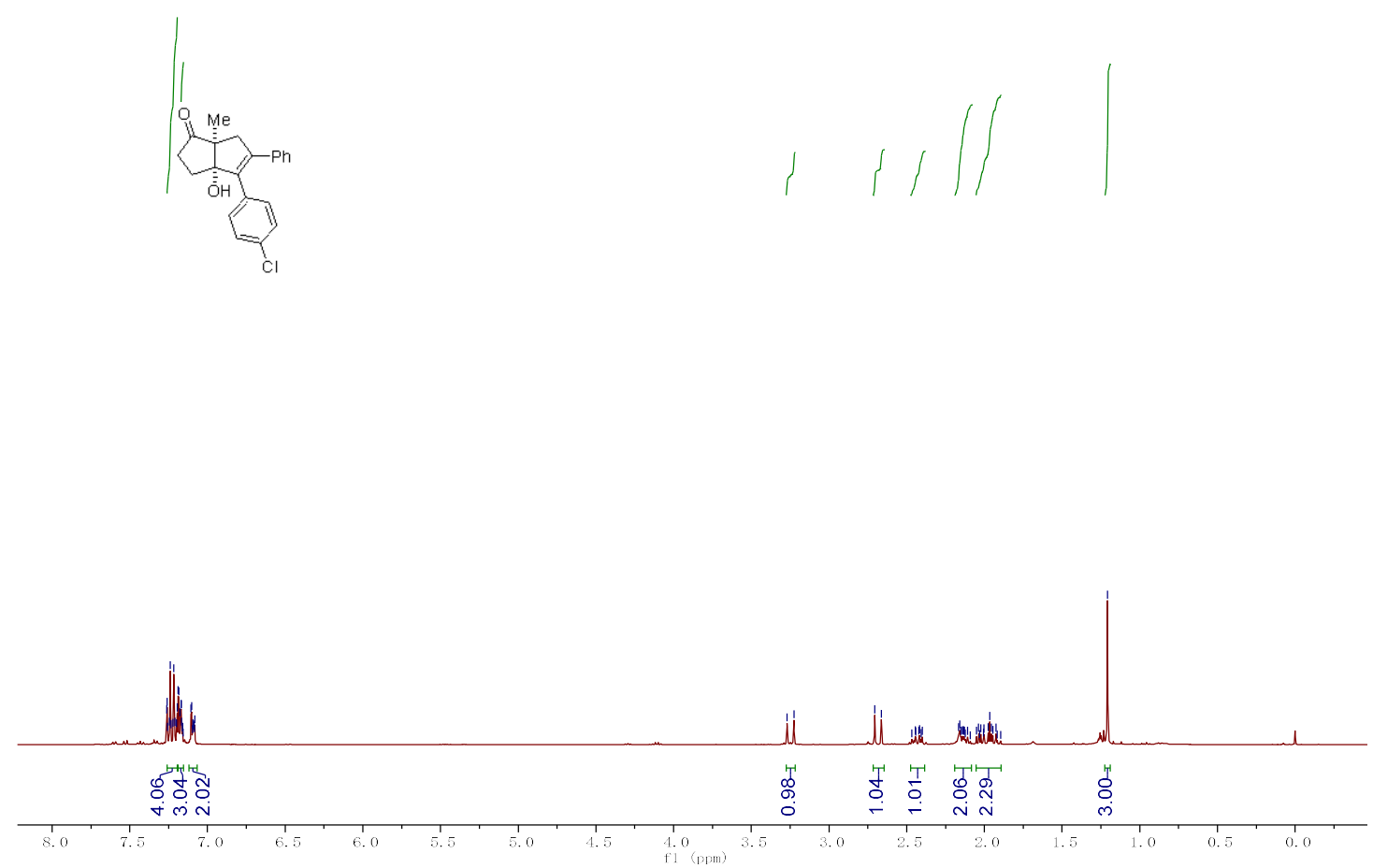

3da (101 MHz for ${ }^{13} \mathrm{C}$ NMR with $\mathrm{CDCl}_{3}$ as solvent)
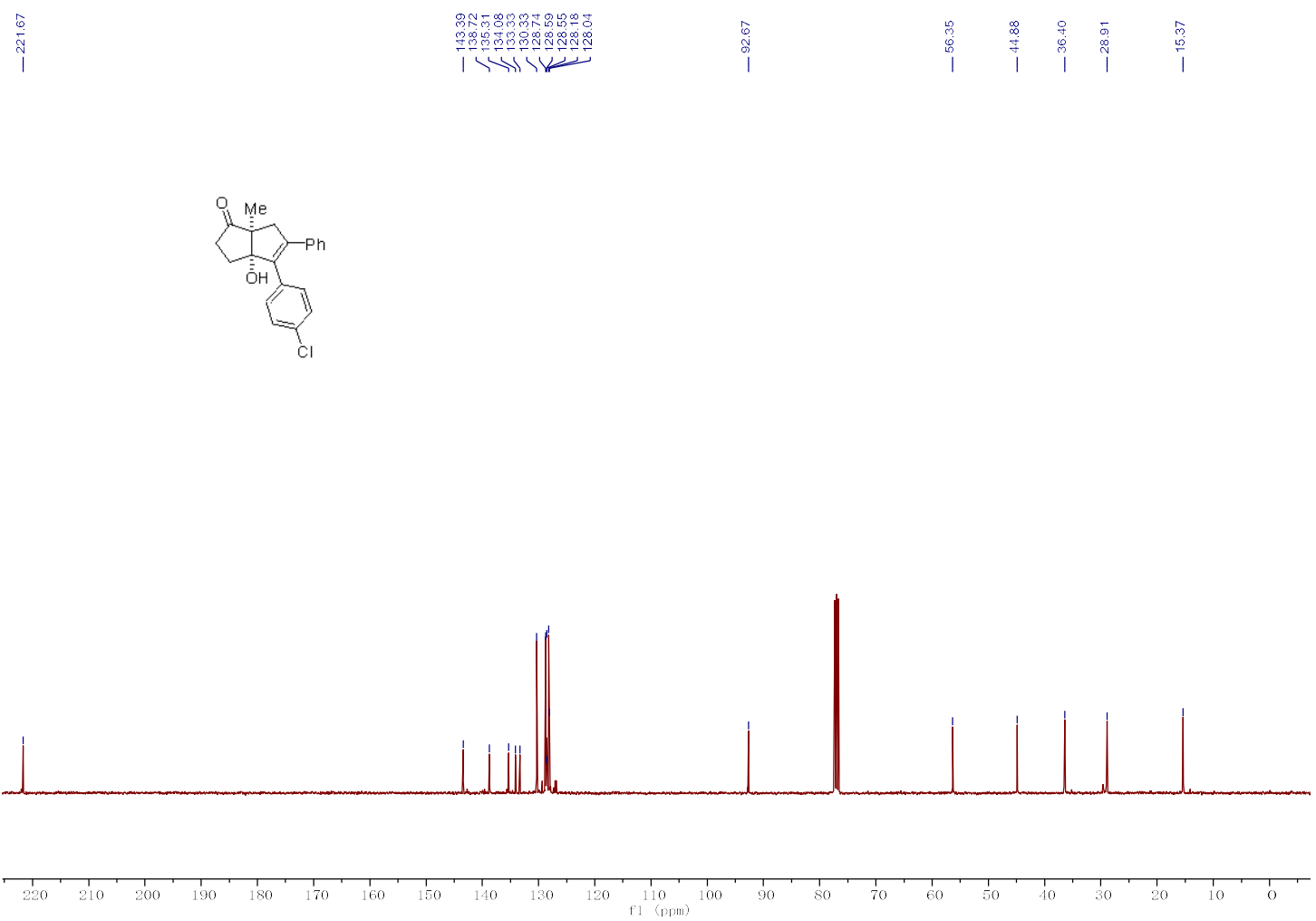
3ea (400 MHz for ${ }^{1} \mathrm{H}$ NMR with $\mathrm{CDCl}_{3}$ as solvent)

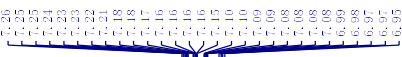
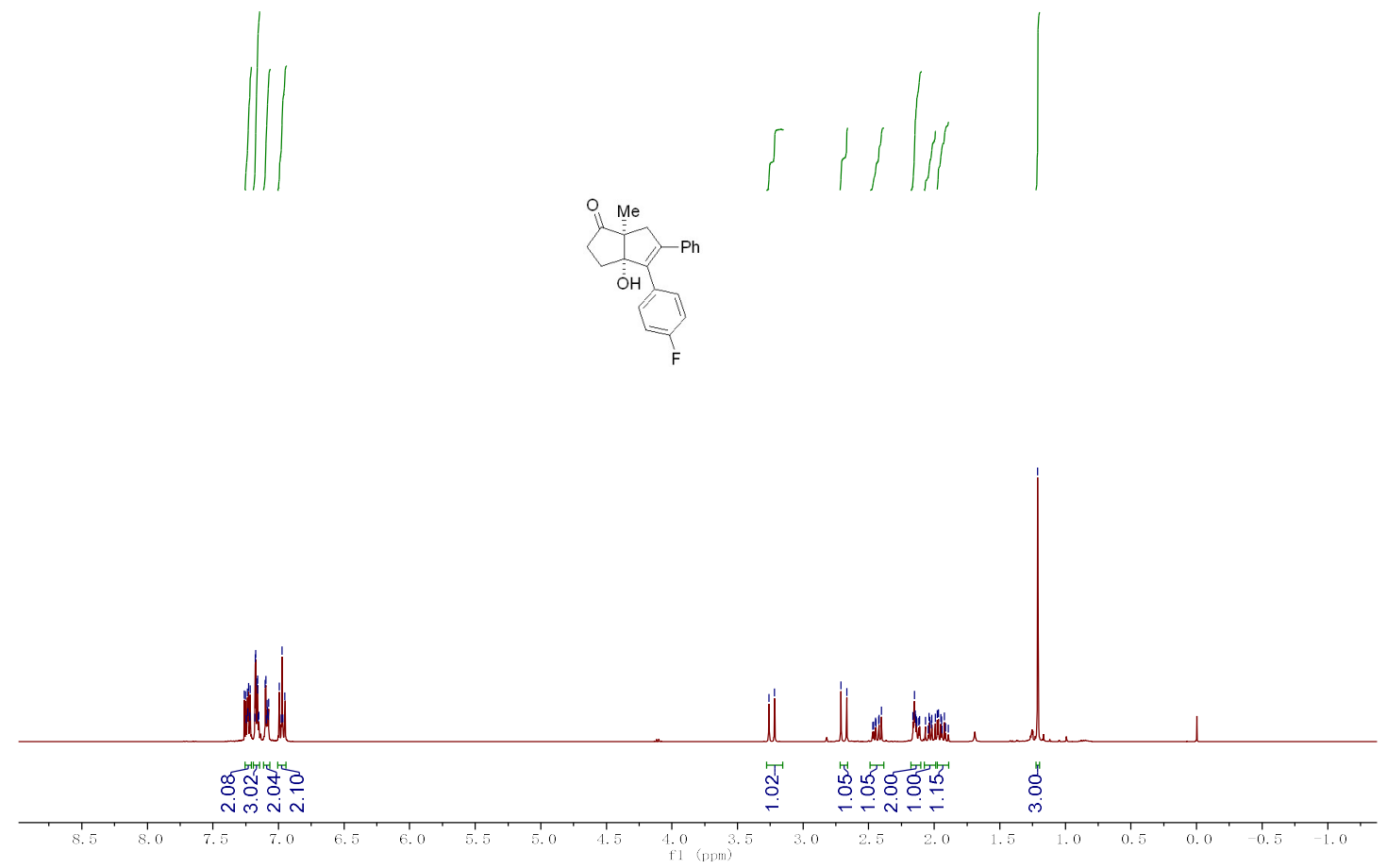

41

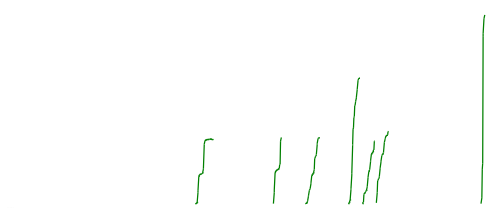

3ea (101 MHz for ${ }^{13} \mathrm{C} \mathrm{NMR}$ with $\mathrm{CDCl}_{3}$ as solvent)

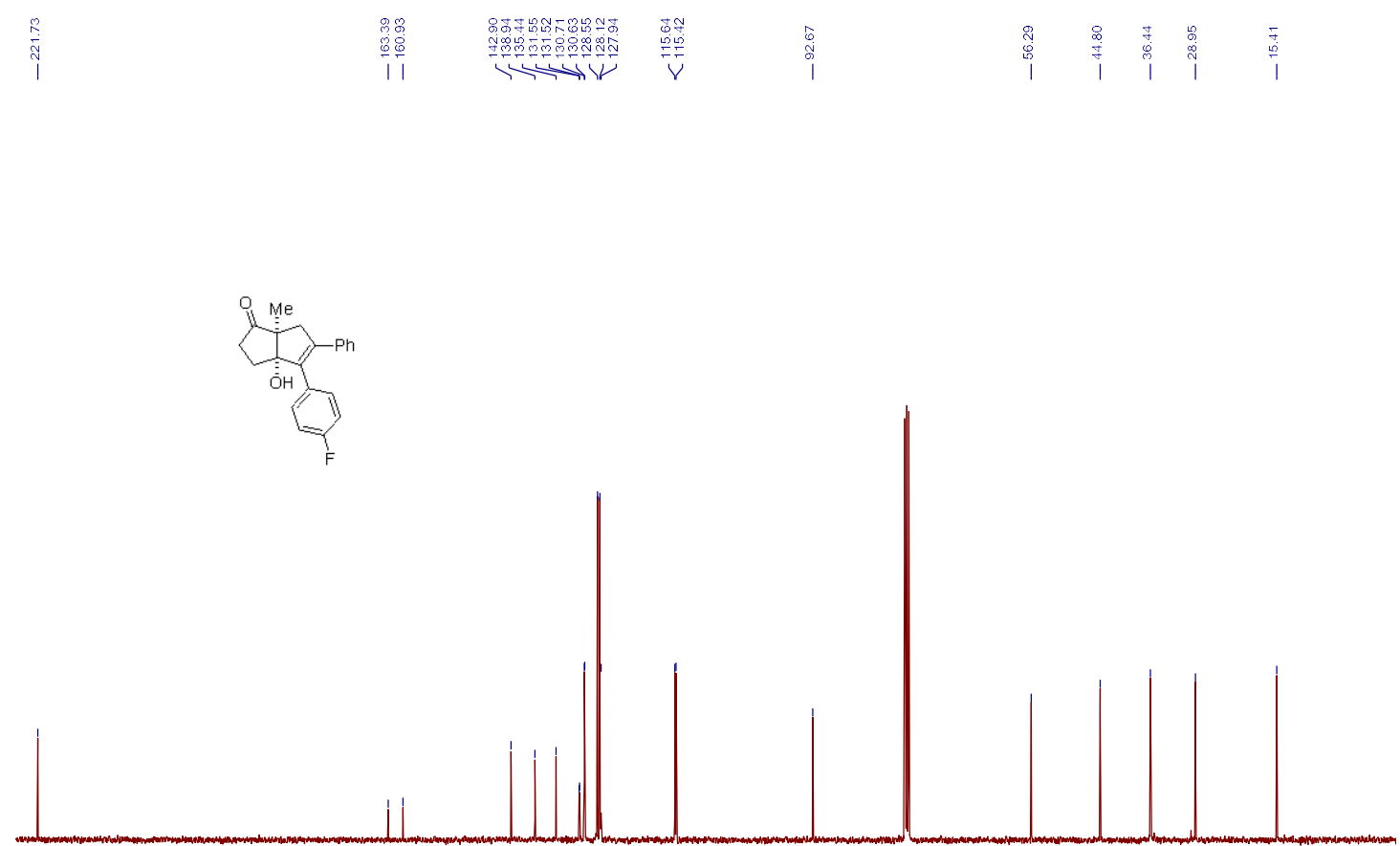

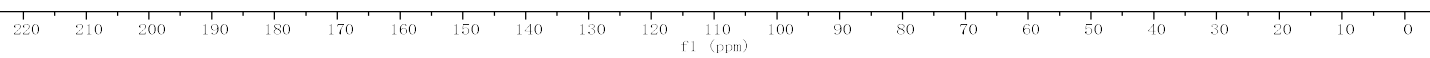


3ea (376 MHz for ${ }^{19} \mathrm{~F} \mathrm{NMR}$ with $\mathrm{CDCl}_{3}$ as solvent)

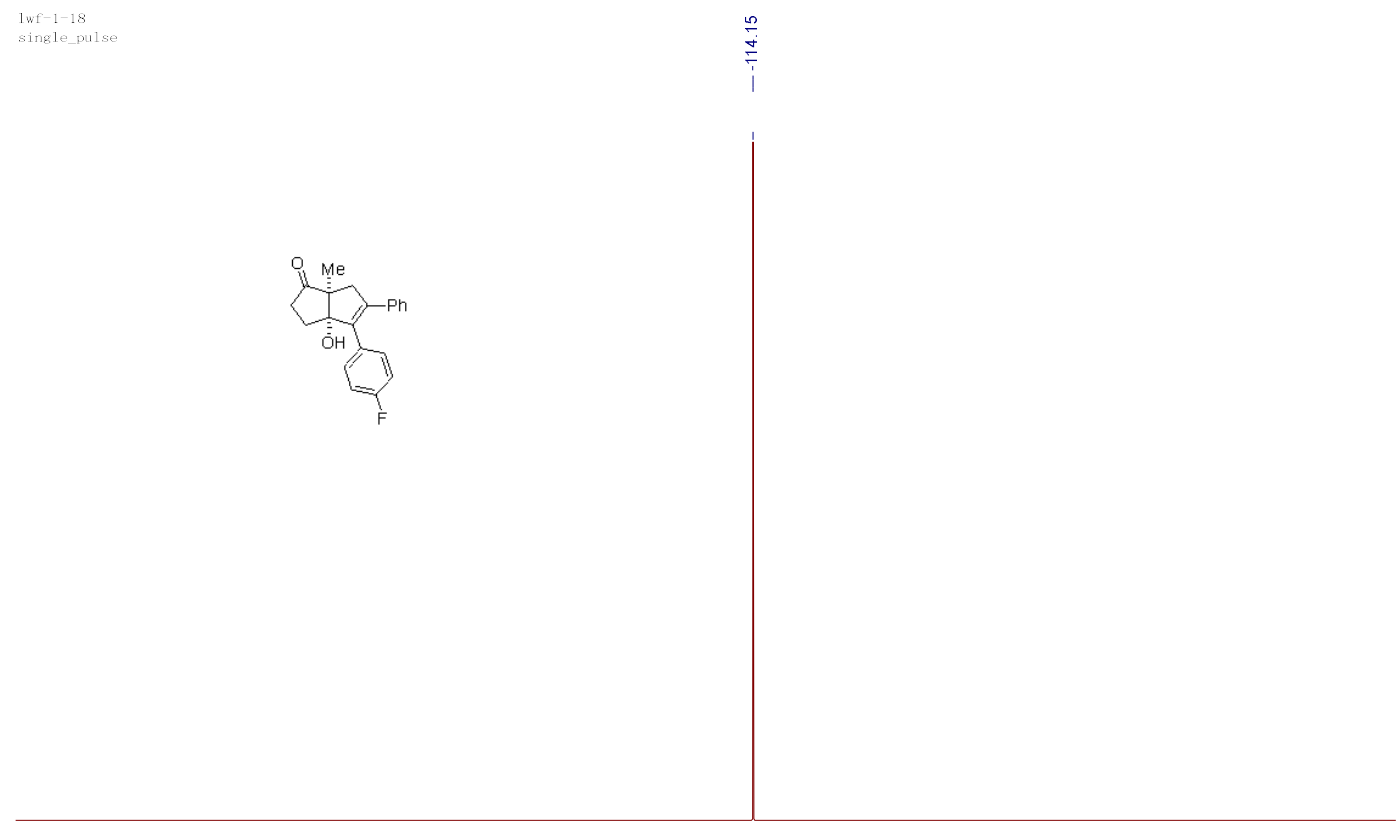


3fa (400 MHz for ${ }^{1} \mathrm{H}$ NMR with $\mathrm{CDCl}_{3}$ as solvent)

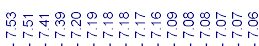

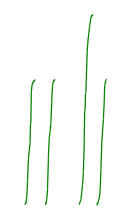

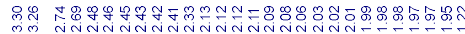
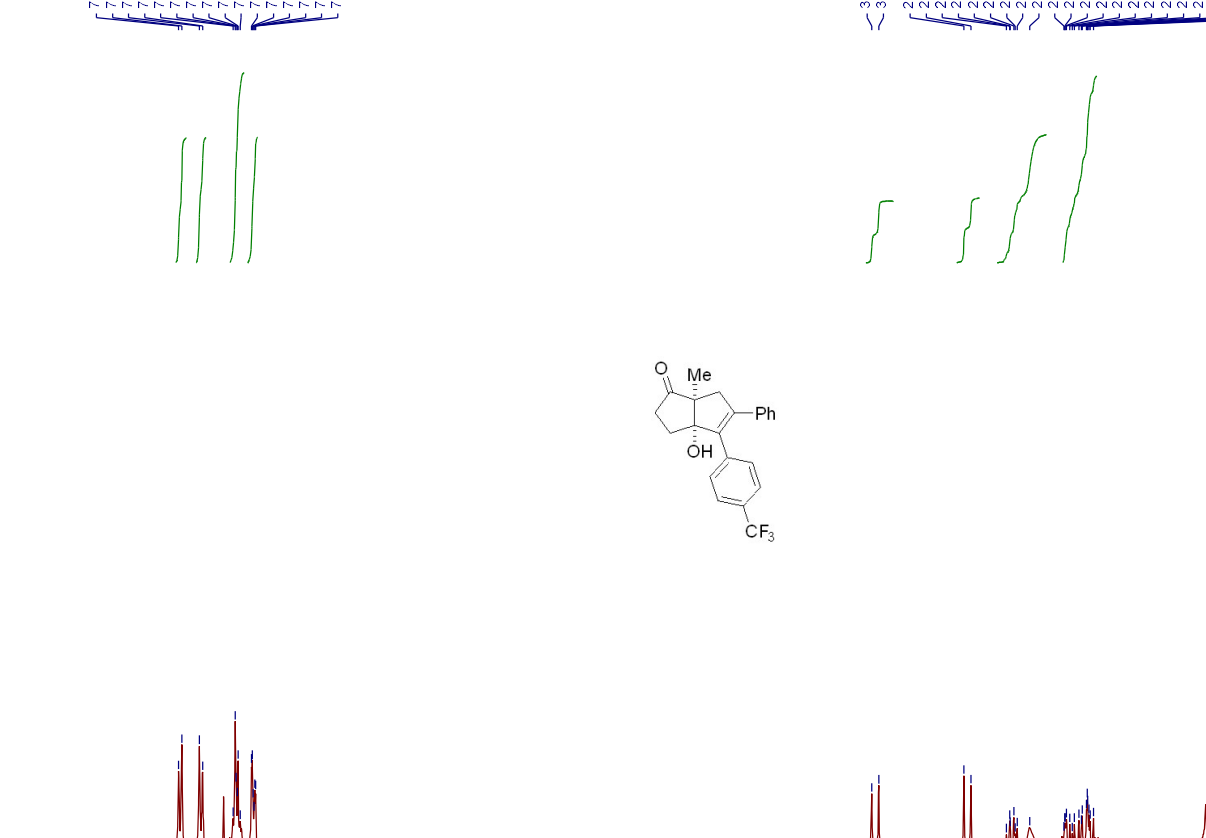

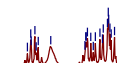

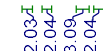

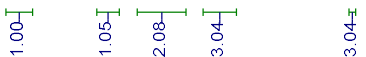

3fa (101 MHz for ${ }^{13} \mathrm{C}$ NMR with $\mathrm{CDCl}_{3}$ as solvent)

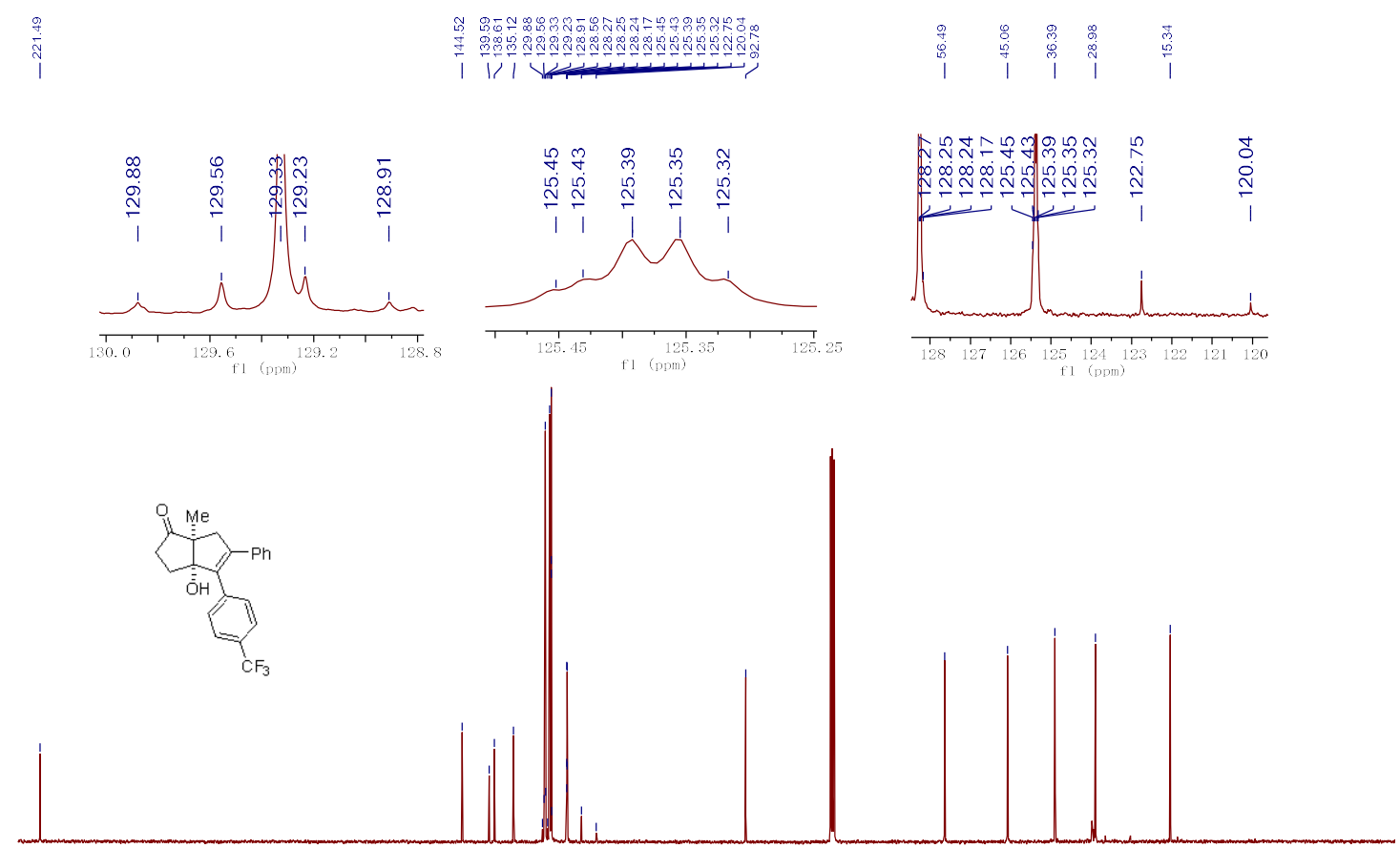


3fa (376 MHz for ${ }^{19} \mathrm{~F} \mathrm{NMR}$ with $\mathrm{CDCl}_{3}$ as solvent)

$\stackrel{?}{?}$

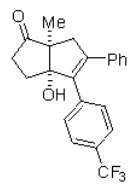


3ga (400 MHz for ${ }^{1} \mathrm{H} \mathrm{NMR}$ with $\mathrm{CDCl}_{3}$ as solvent)

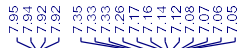

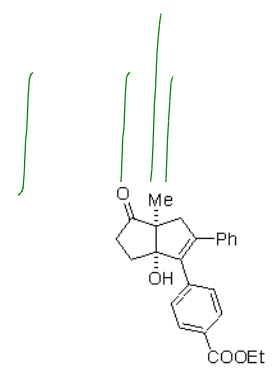

镂㩆

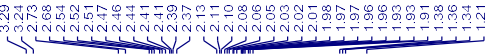

cooet

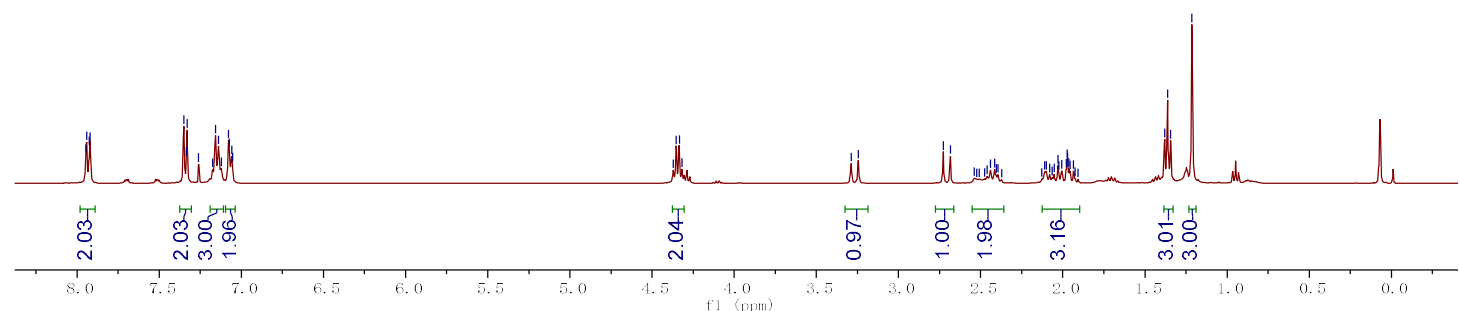

3ga (101 MHz for ${ }^{13} \mathrm{C} \mathrm{NMR}$ with $\mathrm{CDCl}_{3}$ as solvent) ए
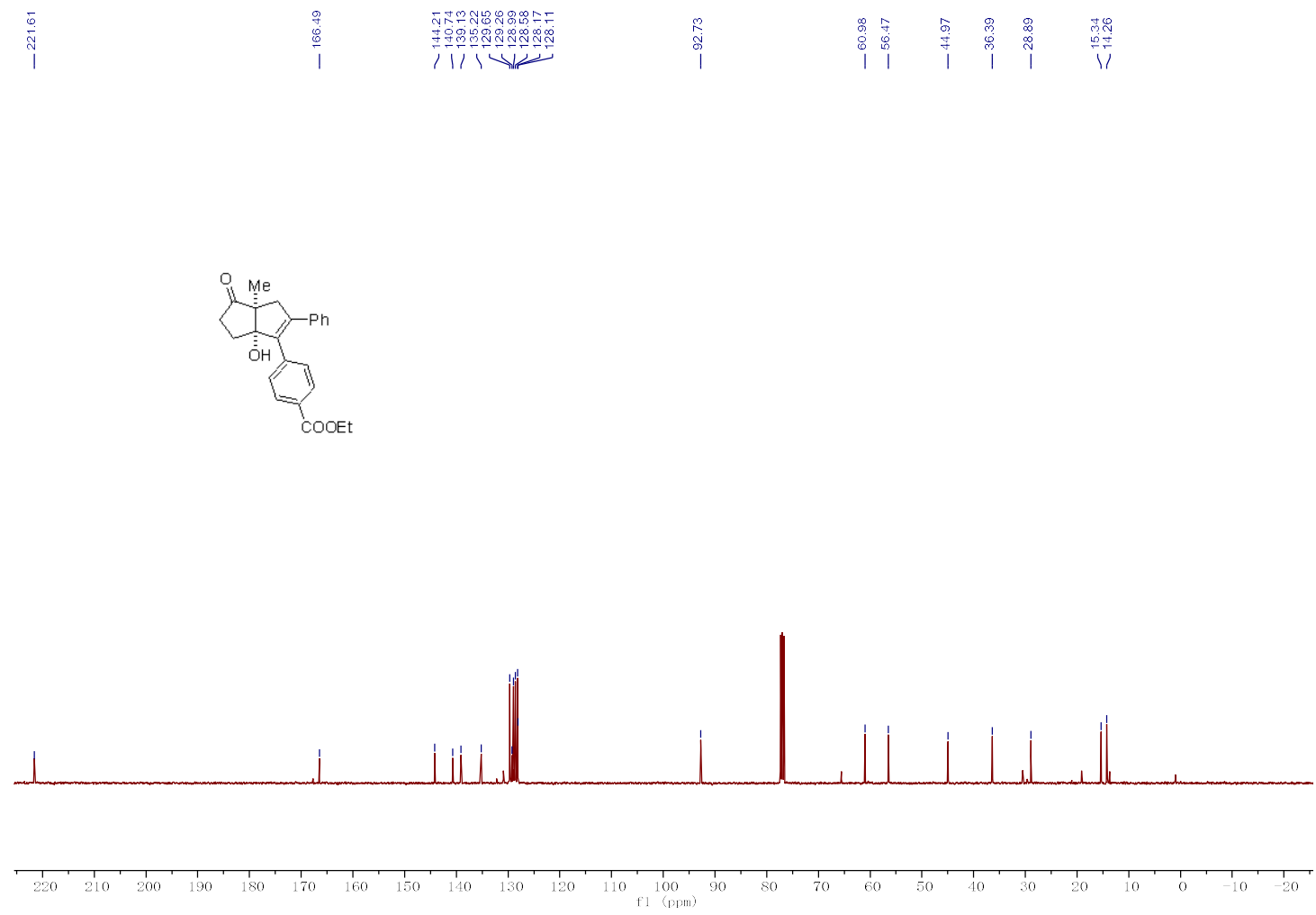
3ha (400 MHz for ${ }^{1} \mathrm{H} \mathrm{NMR}$ with $\mathrm{CDCl}_{3}$ as solvent)

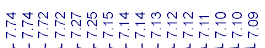

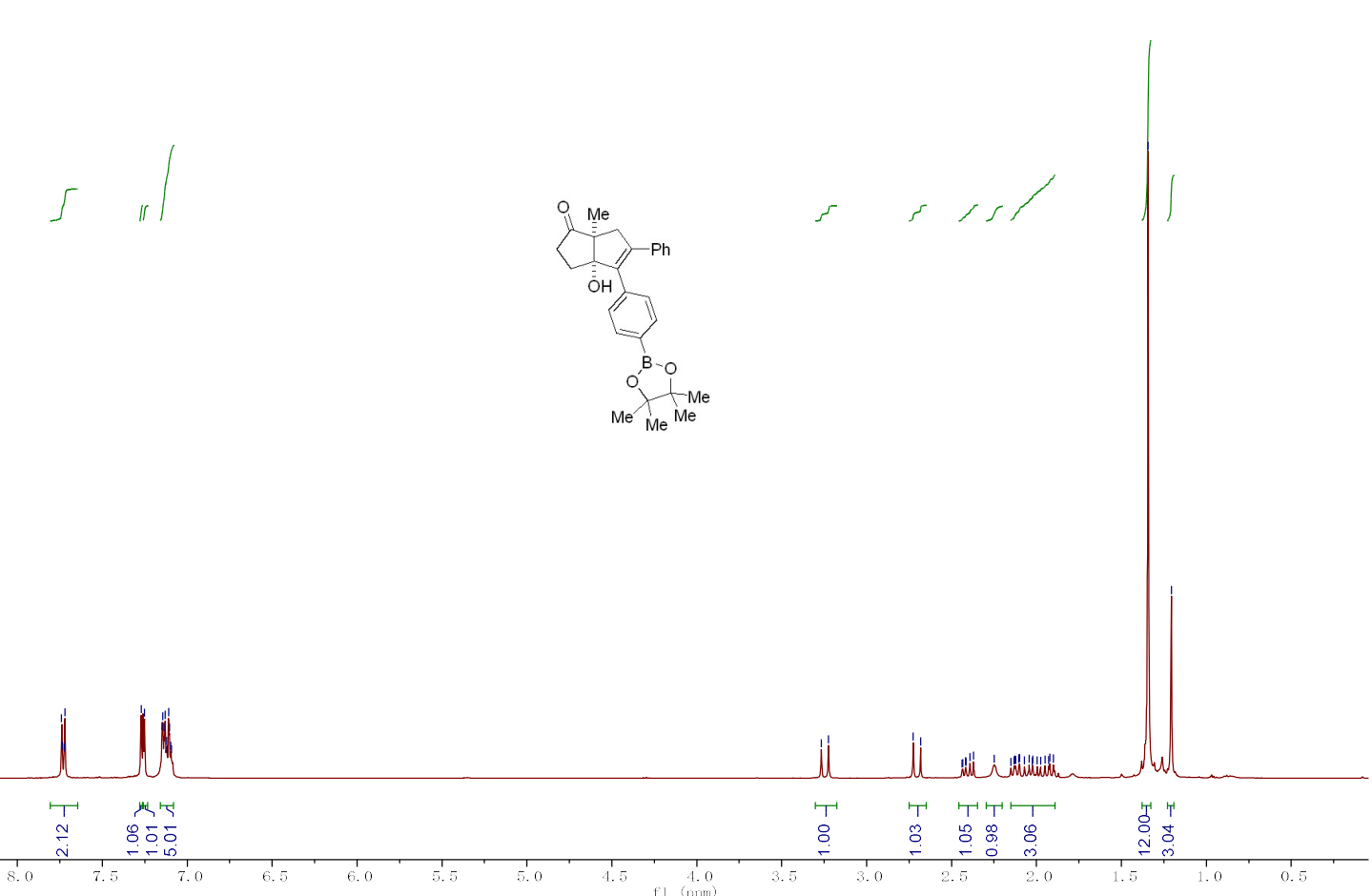

3ha (101 MHz for ${ }^{13} \mathrm{C} \mathrm{NMR}$ with $\mathrm{CDCl}_{3}$ as solvent)

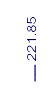
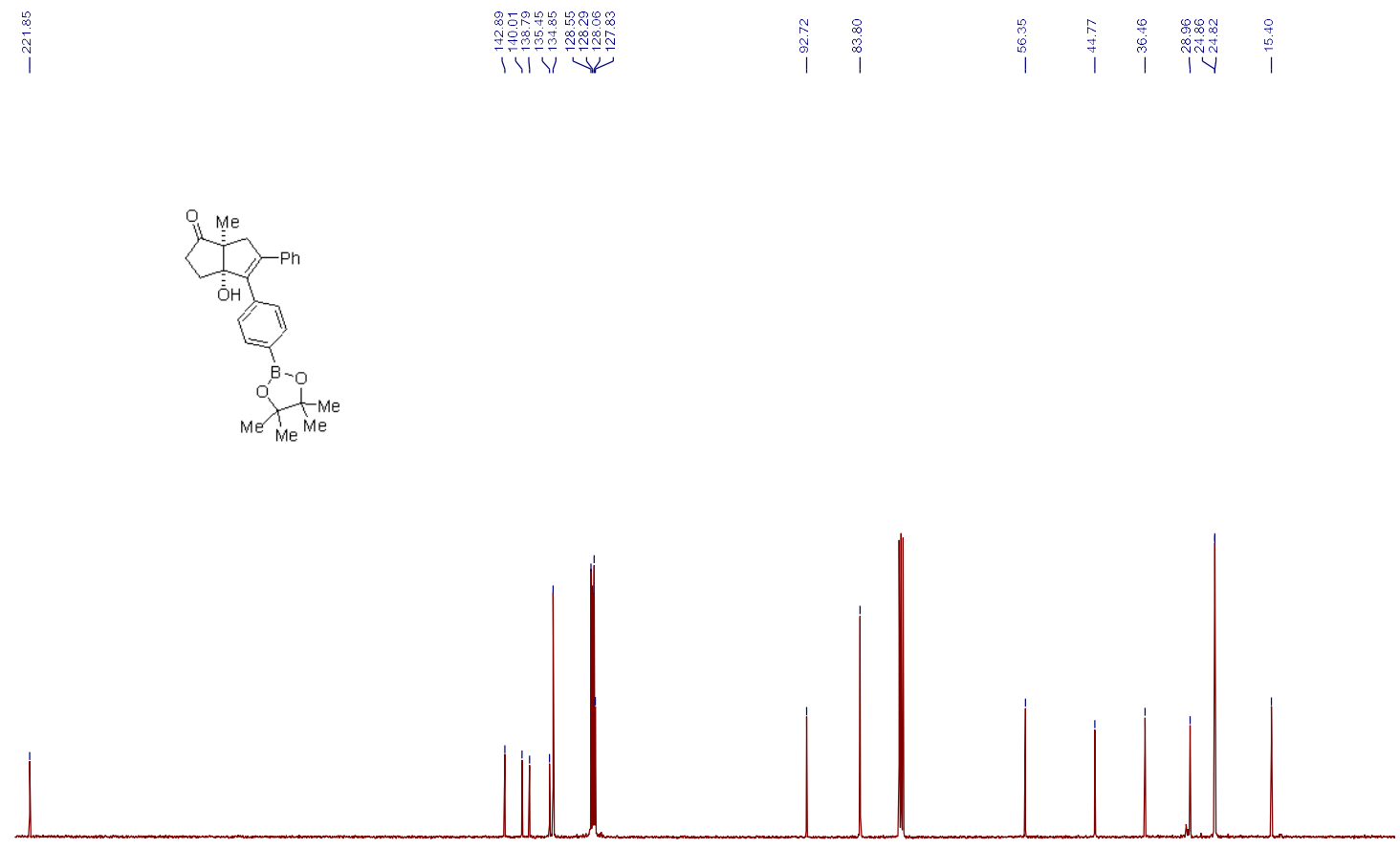
3ia (400 MHz for ${ }^{1} \mathrm{H}$ NMR with $\mathrm{CDCl}_{3}$ as solvent)

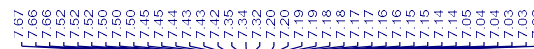
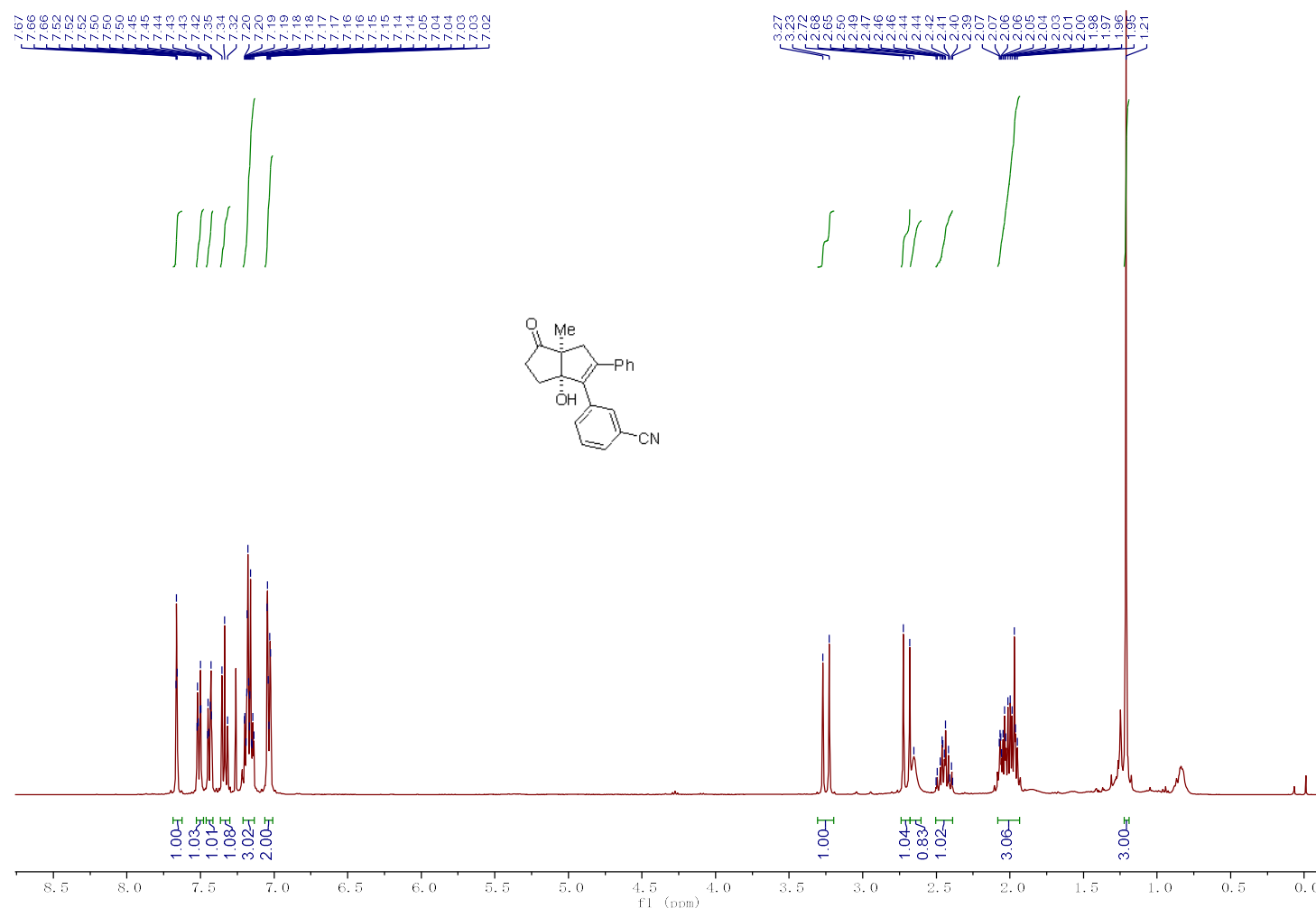

3ia (101 MHz for ${ }^{13} \mathrm{C}$ NMR with $\mathrm{CDCl}_{3}$ as solvent)
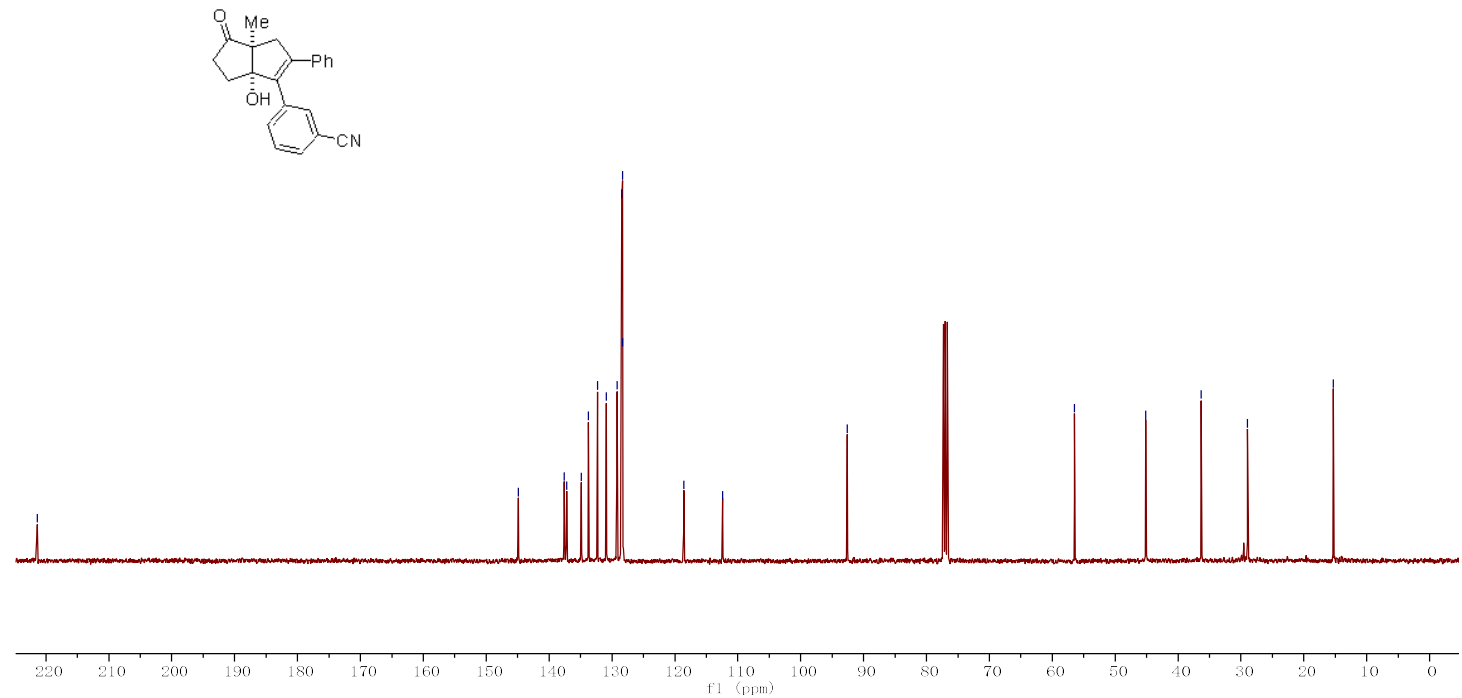
3ja (400 MHz for ${ }^{1} \mathrm{H}$ NMR with $\mathrm{CDCl}_{3}$ as solvent)

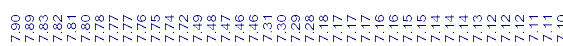

P.

$|1|$
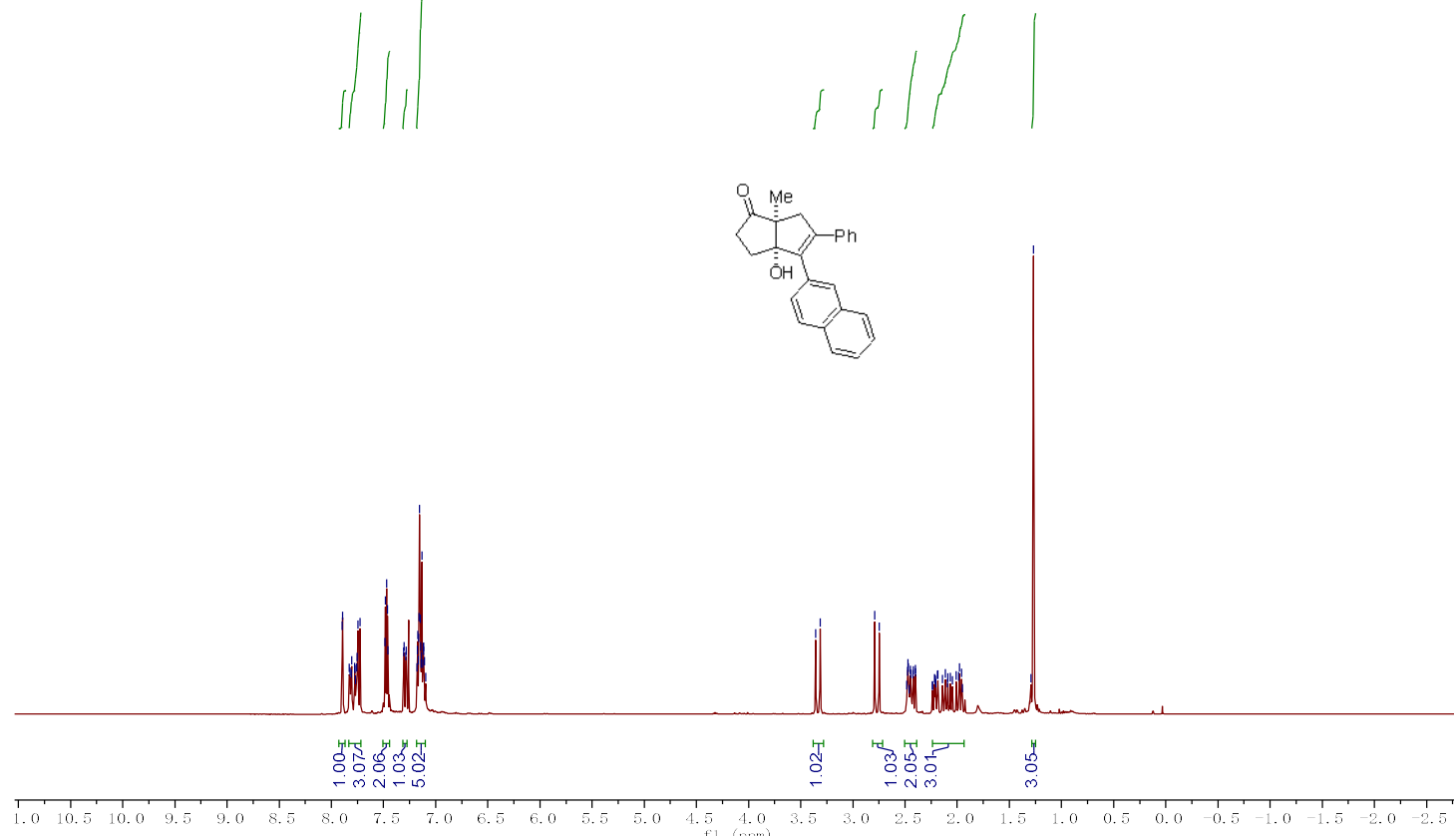

3ja (101 MHz for ${ }^{13} \mathrm{C}$ NMR with $\mathrm{CDCl}_{3}$ as solvent)
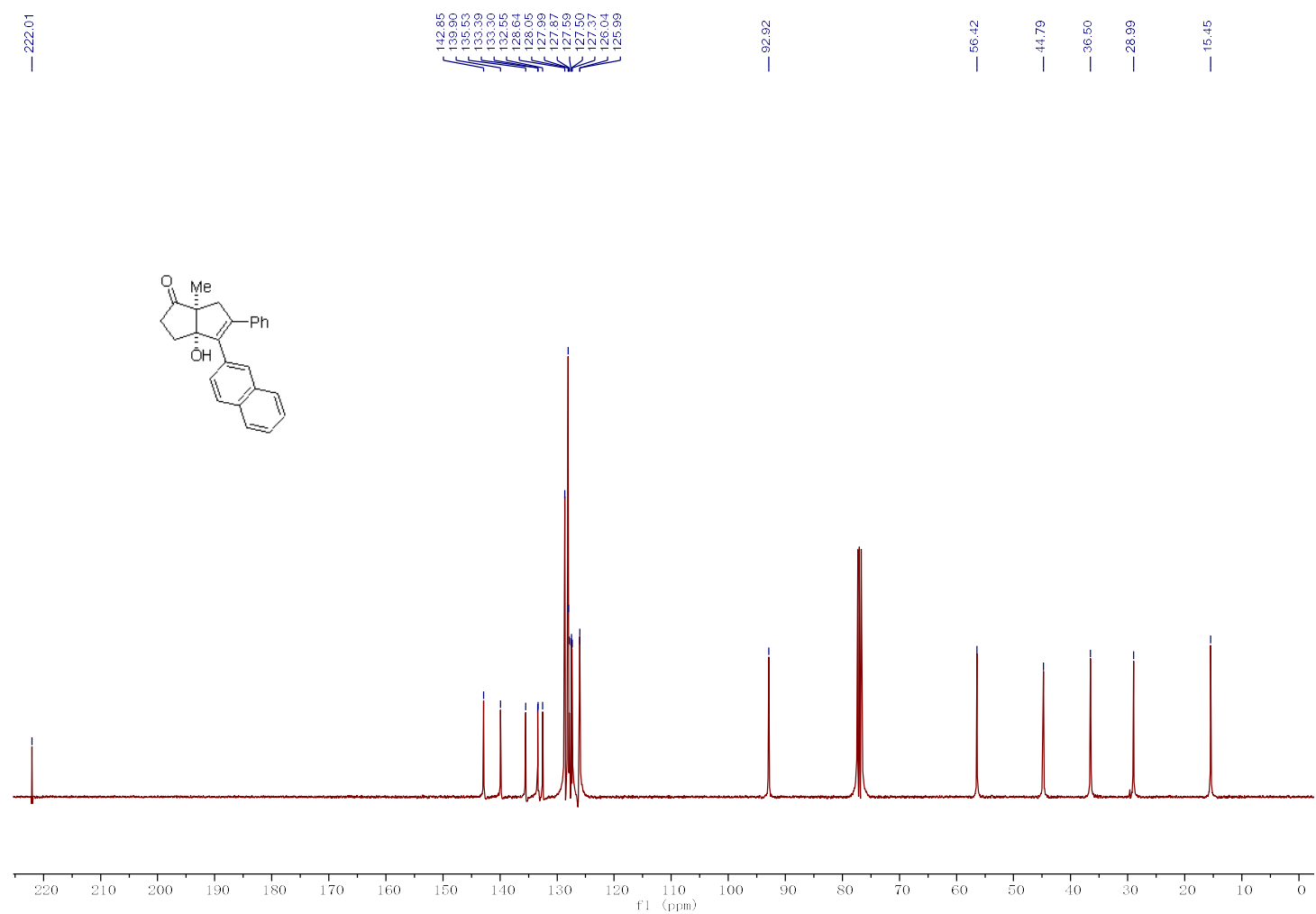
3ka (400 MHz for ${ }^{1} \mathrm{H}$ NMR with $\mathrm{CDCl}_{3}$ as solvent)

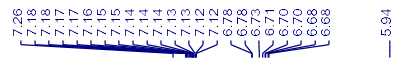

Ra
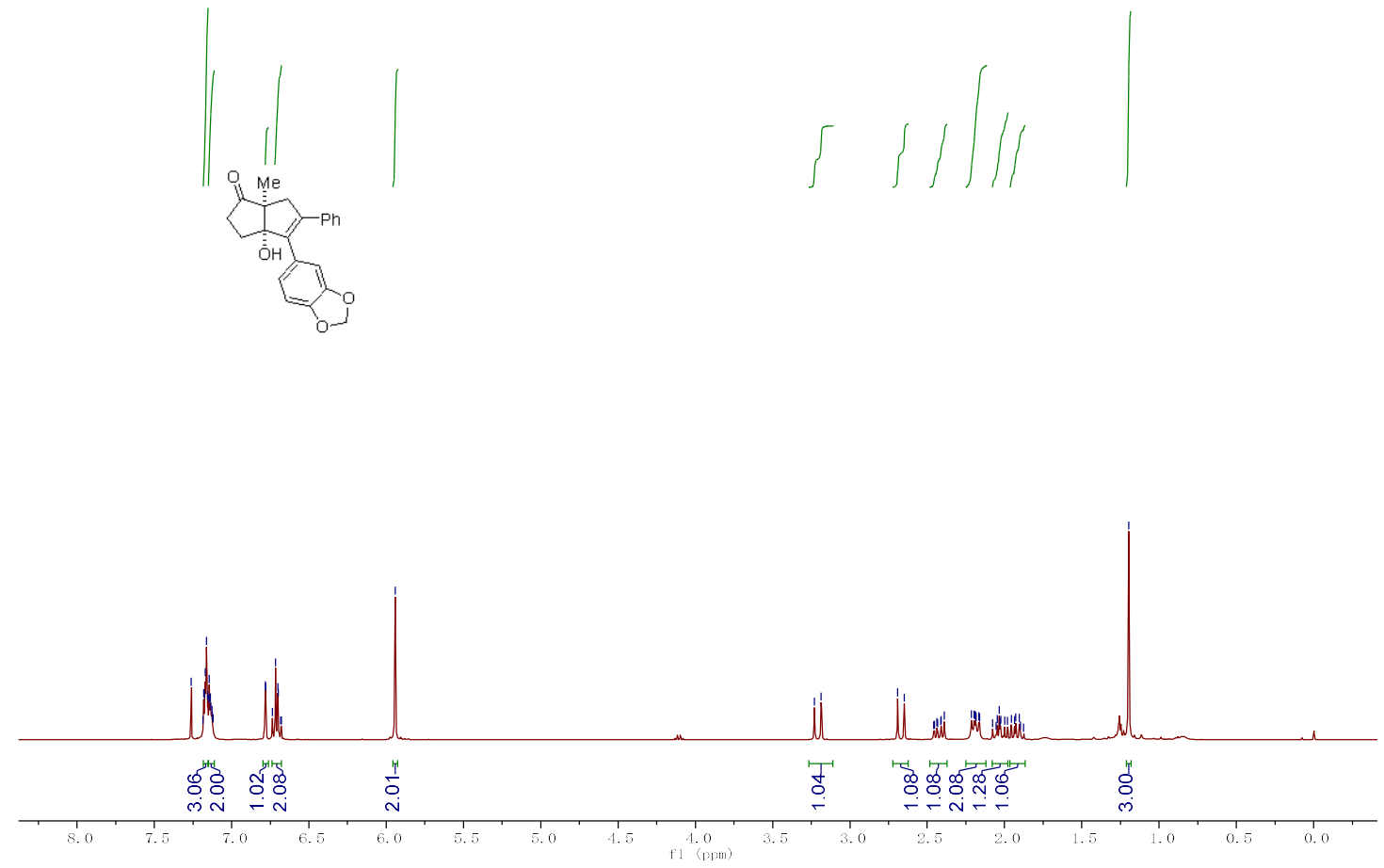

3ka (101 MHz for ${ }^{13} \mathrm{C}$ NMR with $\mathrm{CDCl}_{3}$ as solvent)
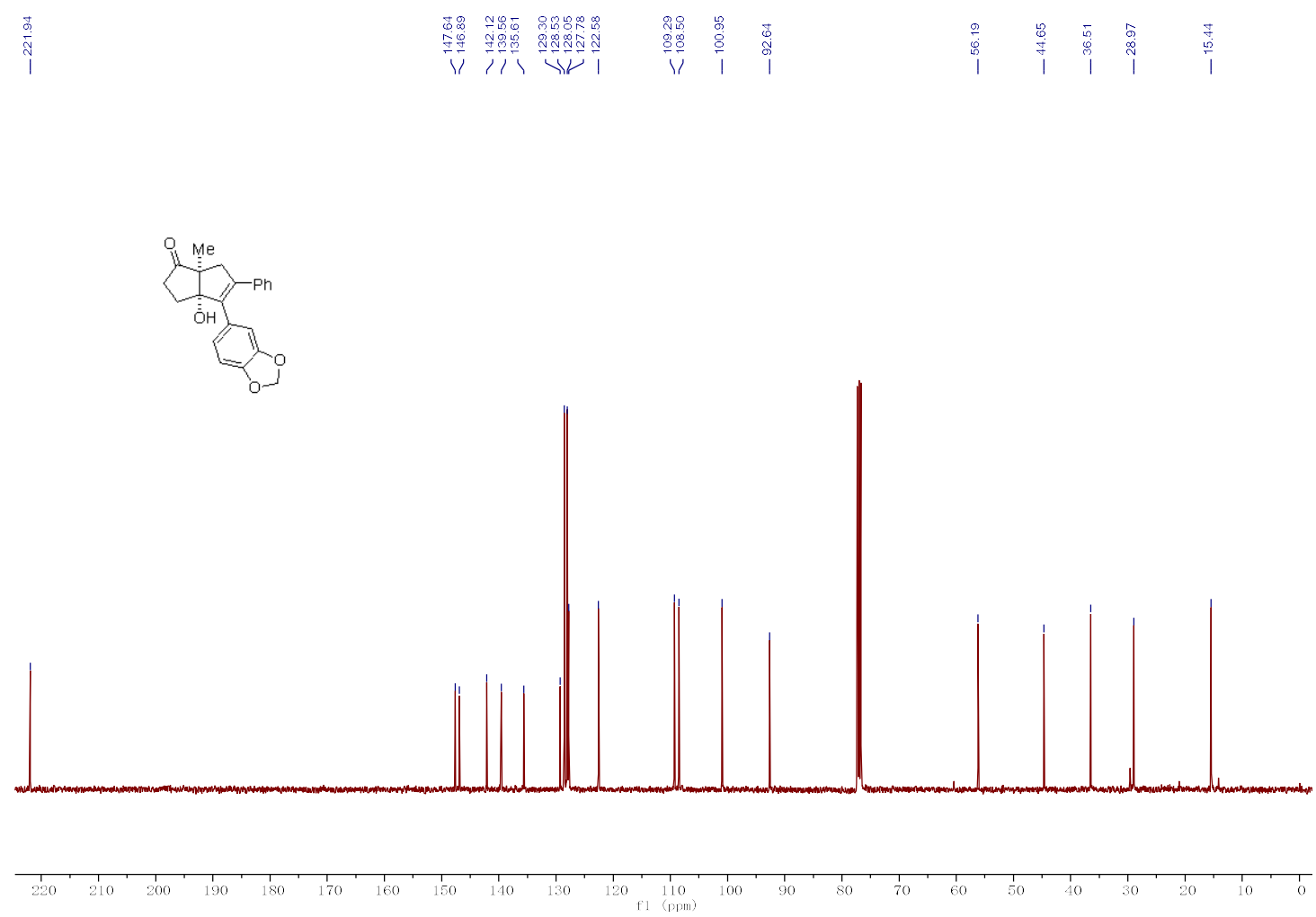
3la (400 MHz for ${ }^{1} \mathrm{H}$ NMR with $\mathrm{CDCl}_{3}$ as solvent)

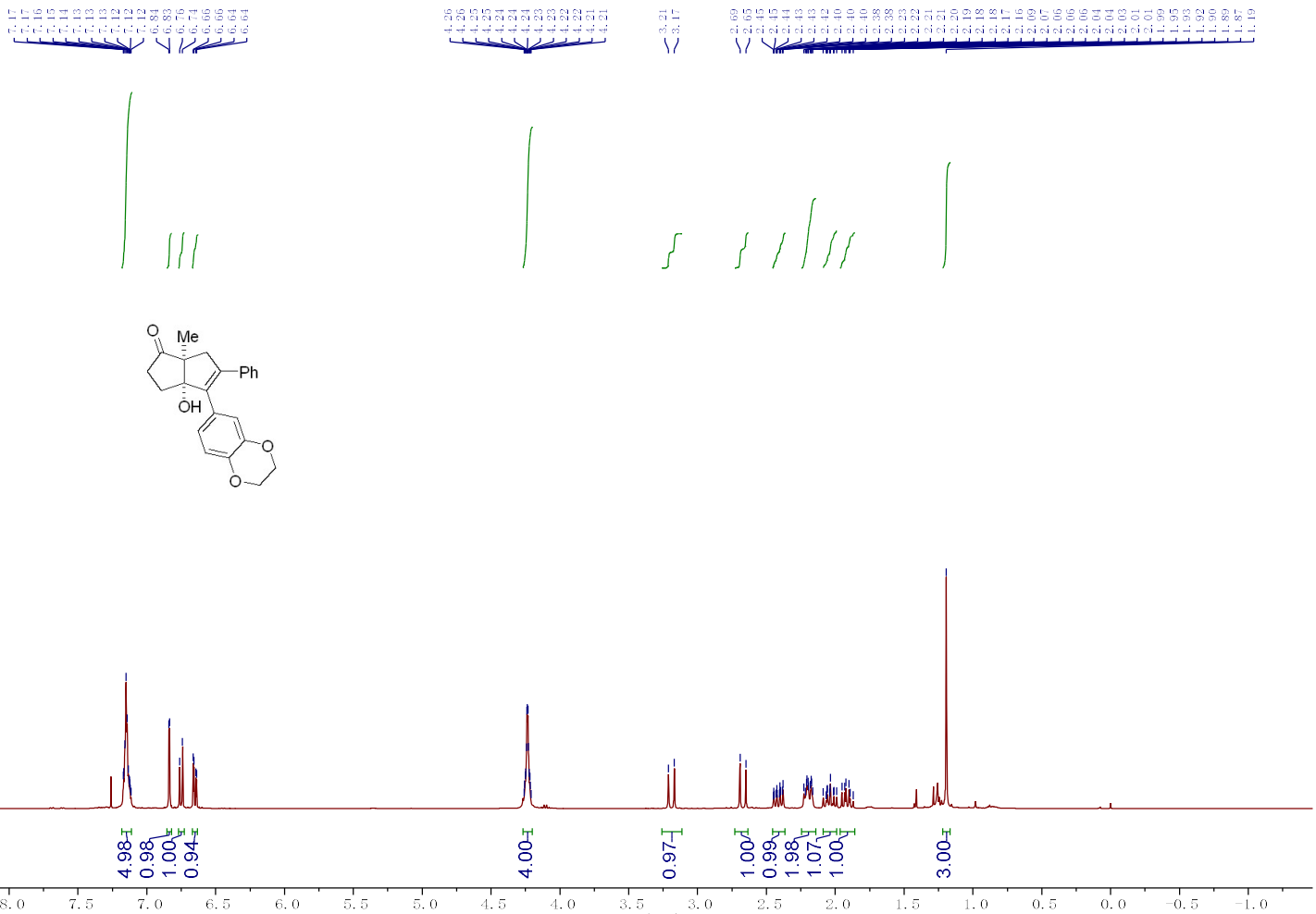

3la (101 MHz for ${ }^{13} \mathrm{C}$ NMR with $\mathrm{CDCl}_{3}$ as solvent)

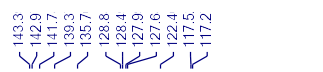

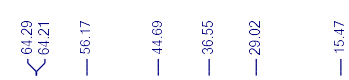

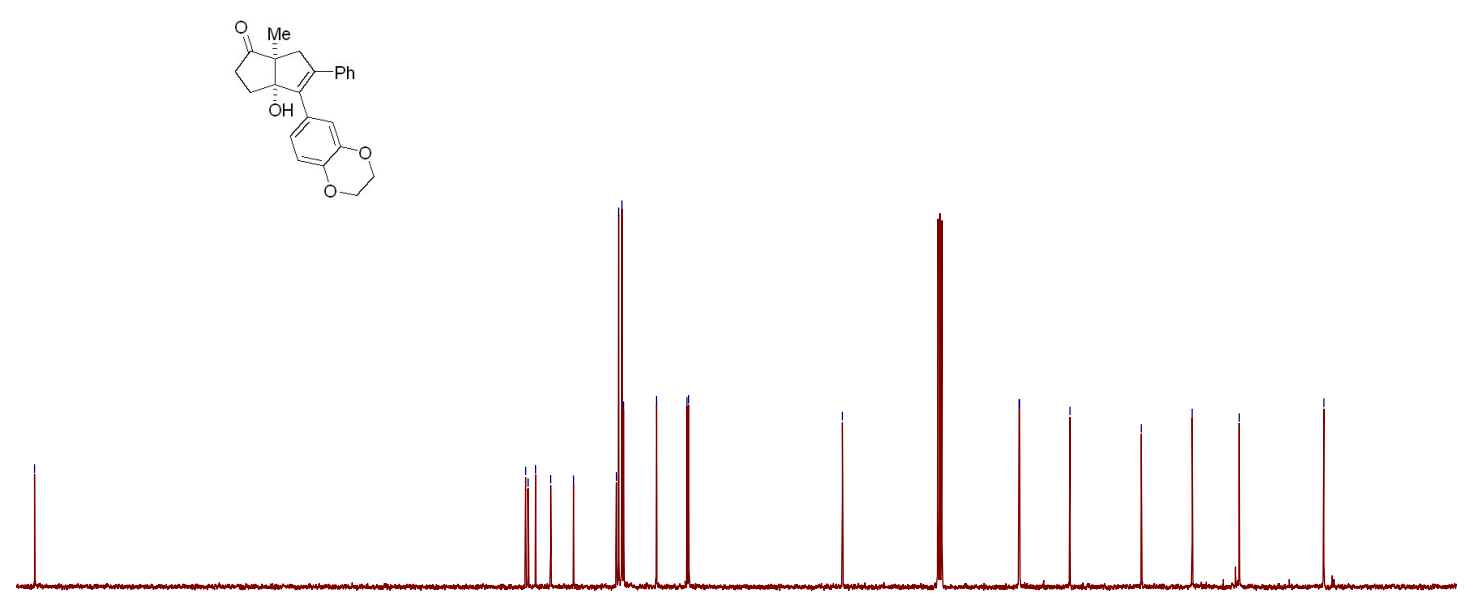


3ma (400 MHz for ${ }^{1} \mathrm{H}$ NMR with $\mathrm{CDCl}_{3}$ as solvent)

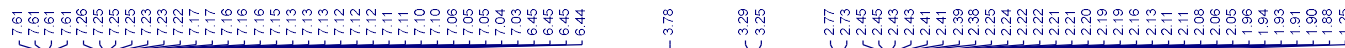
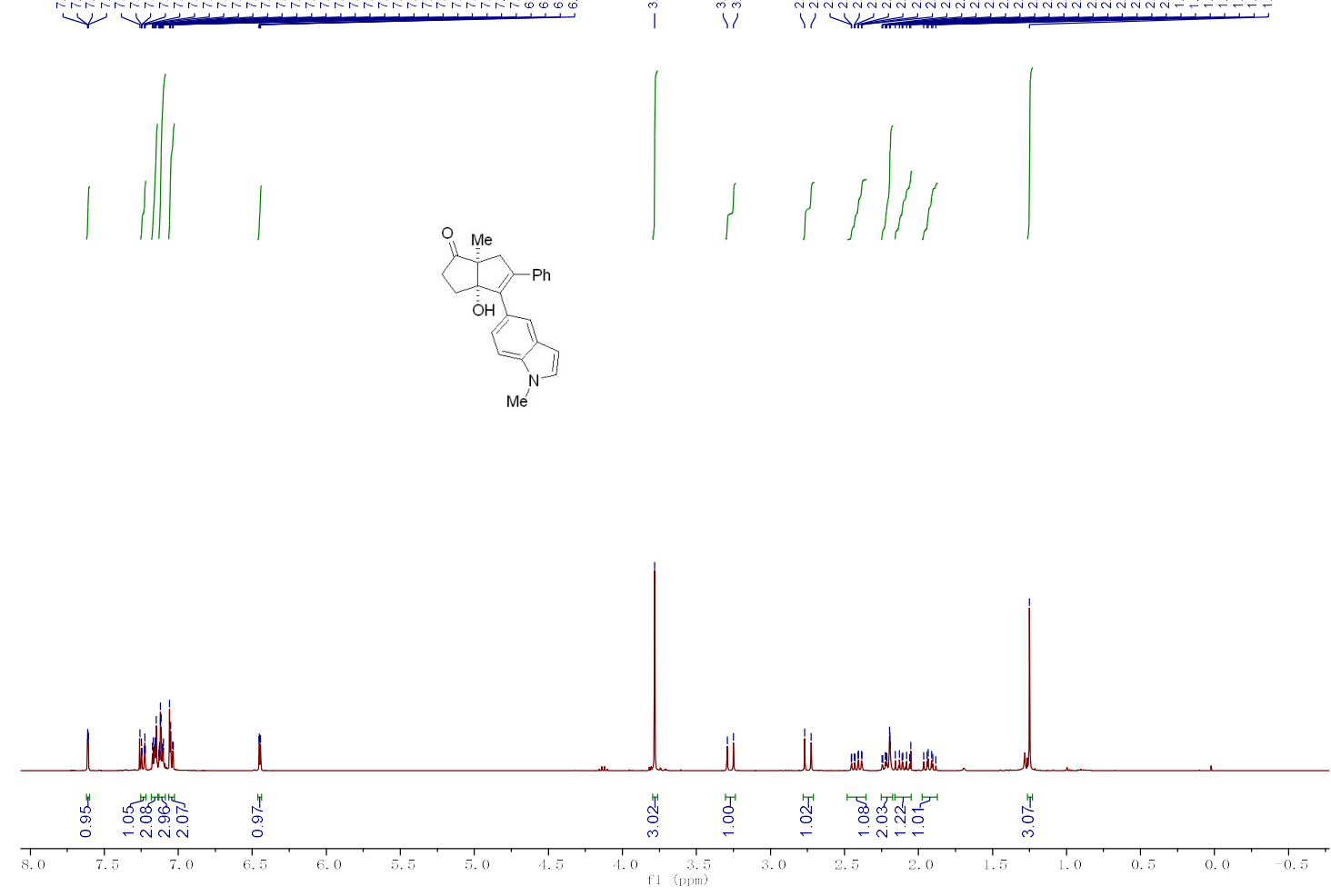

3ma (101 MHz for ${ }^{13} \mathrm{C} \mathrm{NMR}$ with $\mathrm{CDCl}_{3}$ as solvent)

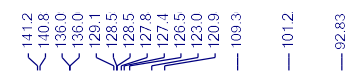
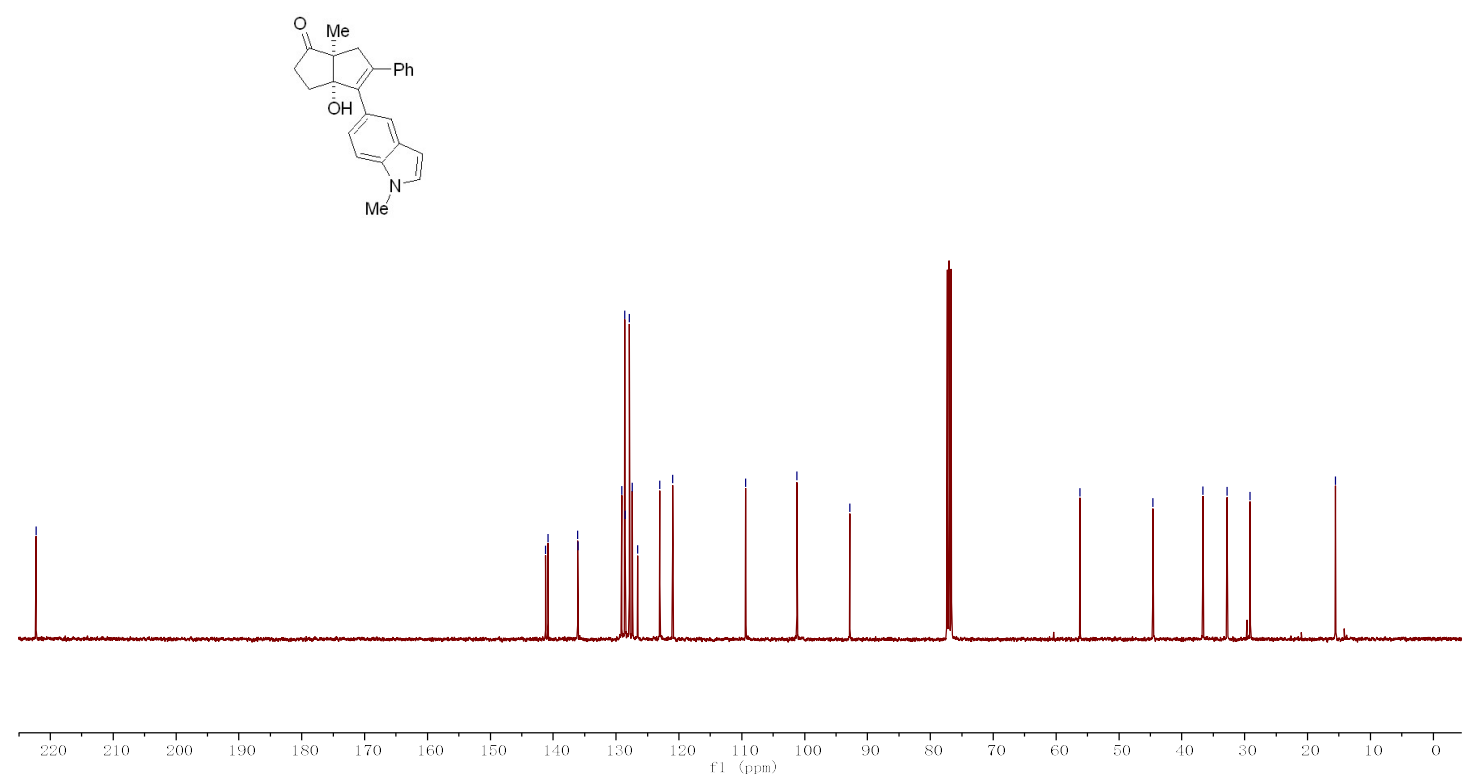
3na (400 MHz for ${ }^{1} \mathrm{H} \mathrm{NMR}$ with $\mathrm{CDCl}_{3}$ as solvent)

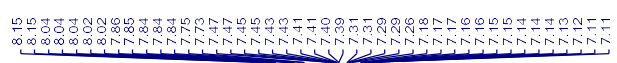

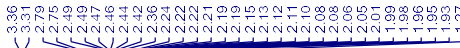

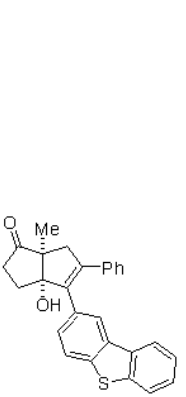

|| || ||
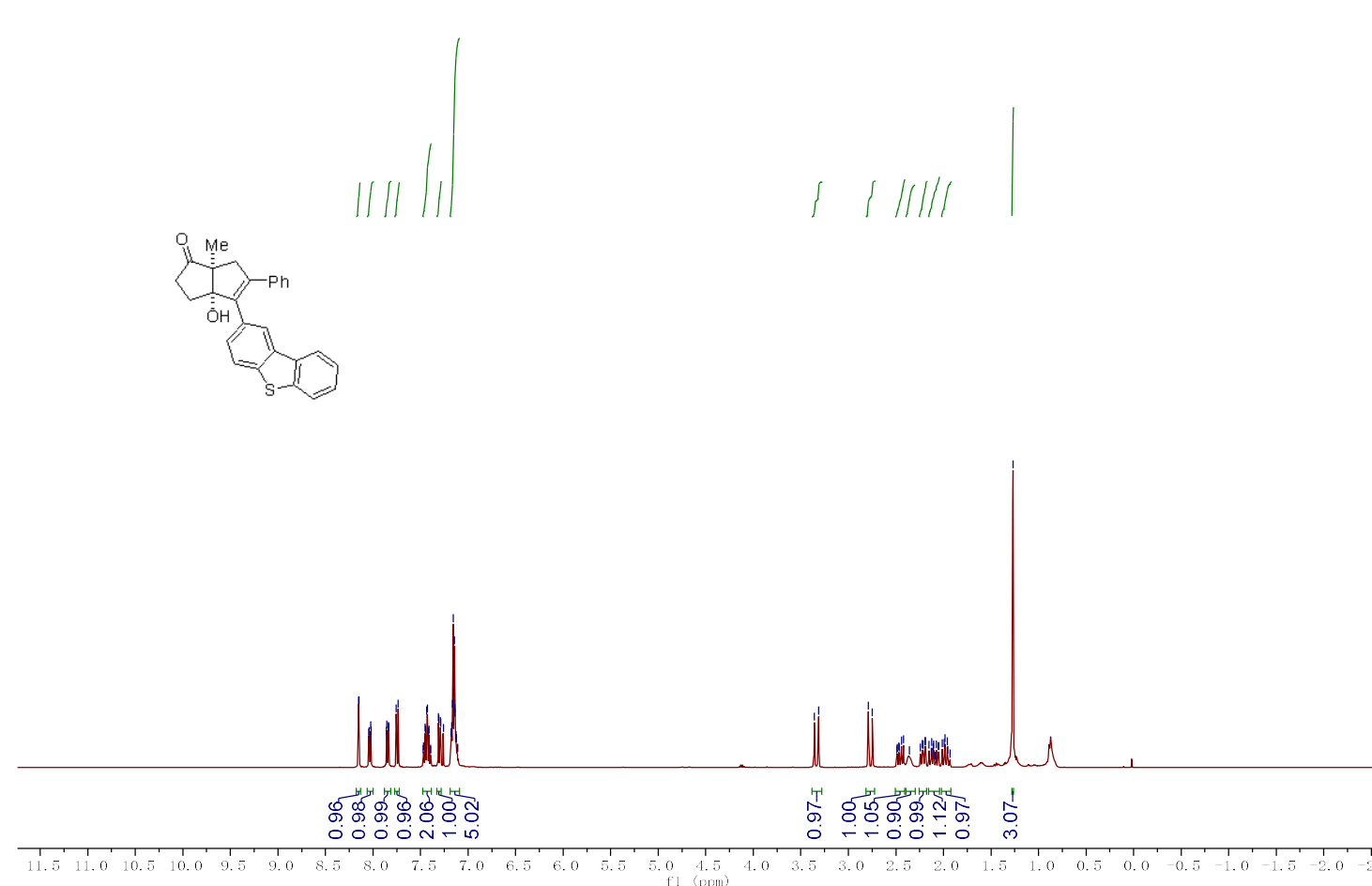

3na (101 MHz for ${ }^{13} \mathrm{C} \mathrm{NMR}$ with $\mathrm{CDCl}_{3}$ as solvent)

$$
\text { 길 }
$$

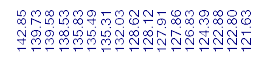

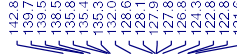
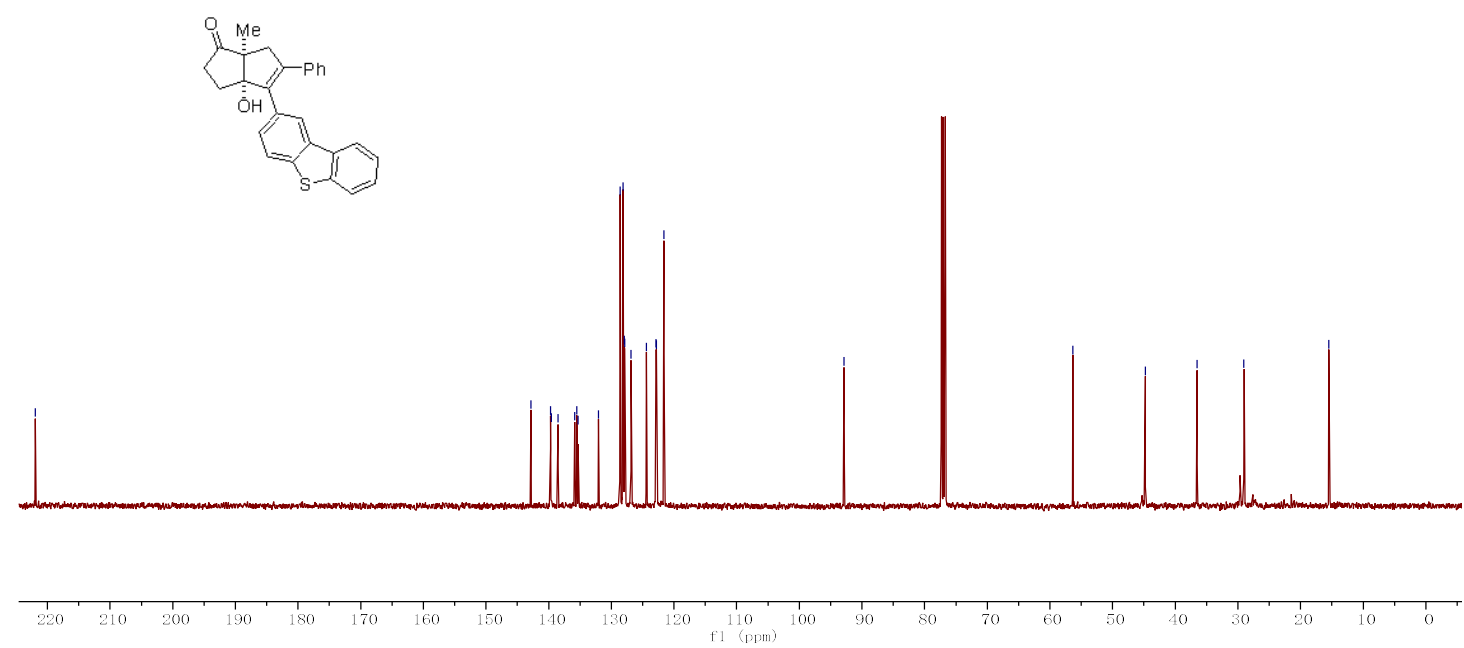
3oa (400 MHz for ${ }^{1} \mathrm{H} \mathrm{NMR}$ with $\mathrm{CDCl}_{3}$ as solvent)

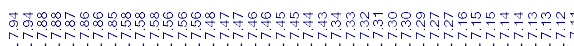

\|\|$\|$

th

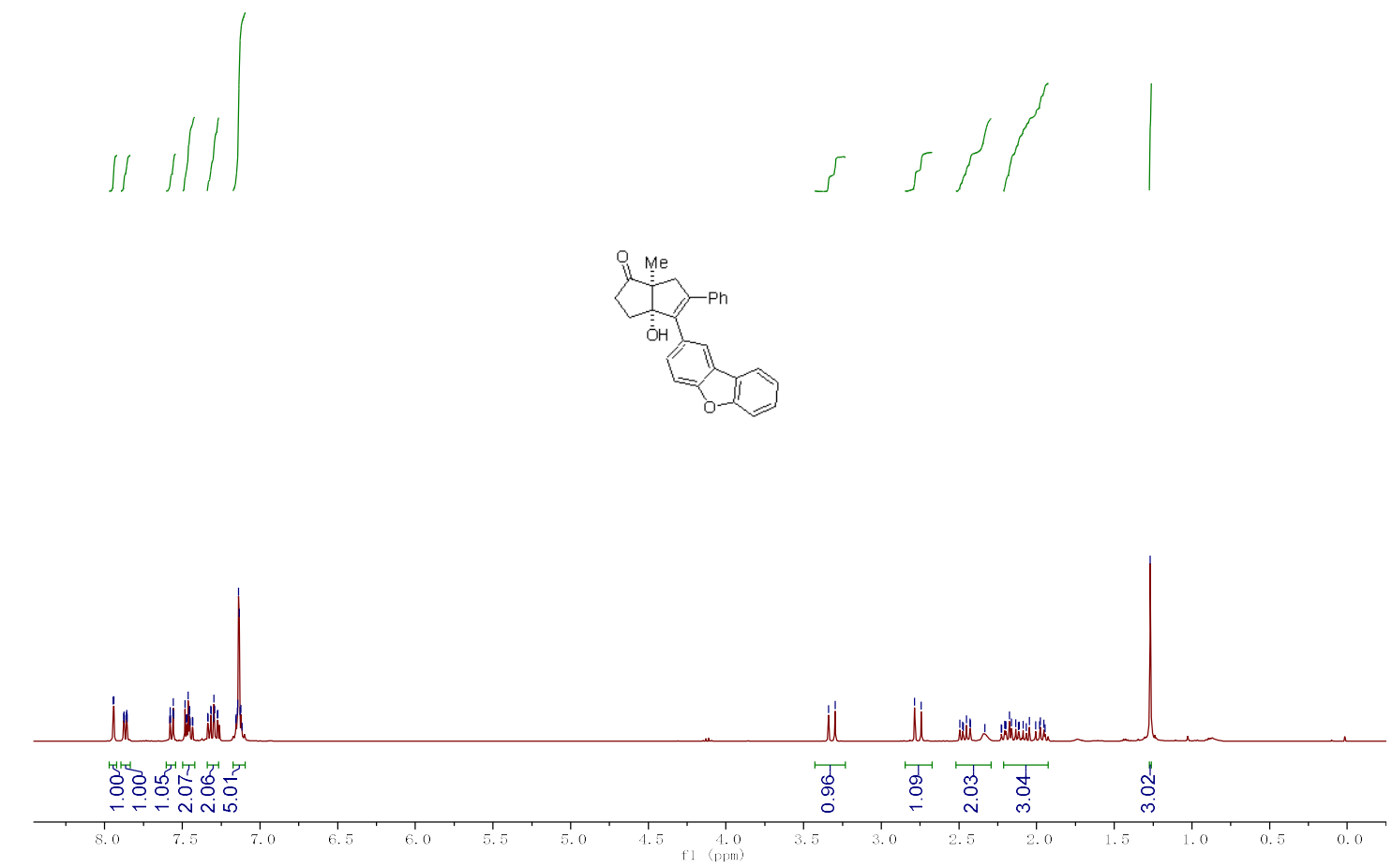

3oa (101 MHz for ${ }^{13} \mathrm{C} \mathrm{NMR}$ with $\mathrm{CDCl}_{3}$ as solvent)
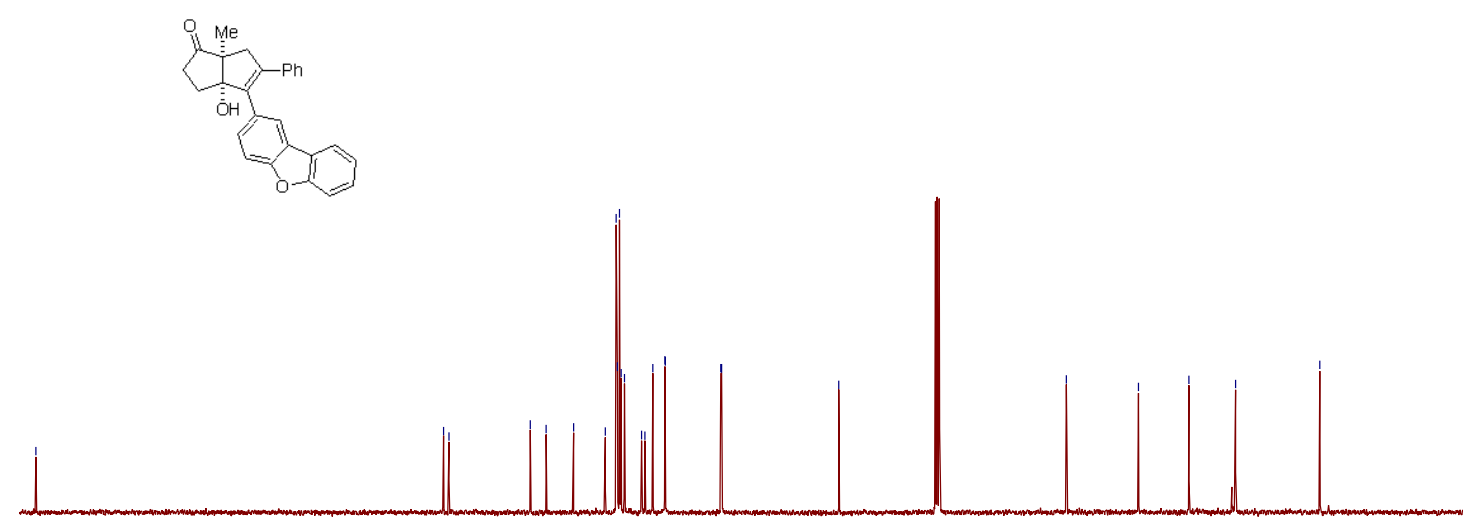
3pa (400 MHz for ${ }^{1} \mathrm{H} \mathrm{NMR}$ with $\mathrm{CDCl}_{3}$ as solvent)

V

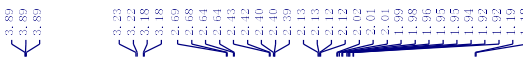

$\int \Gamma \mid \int$
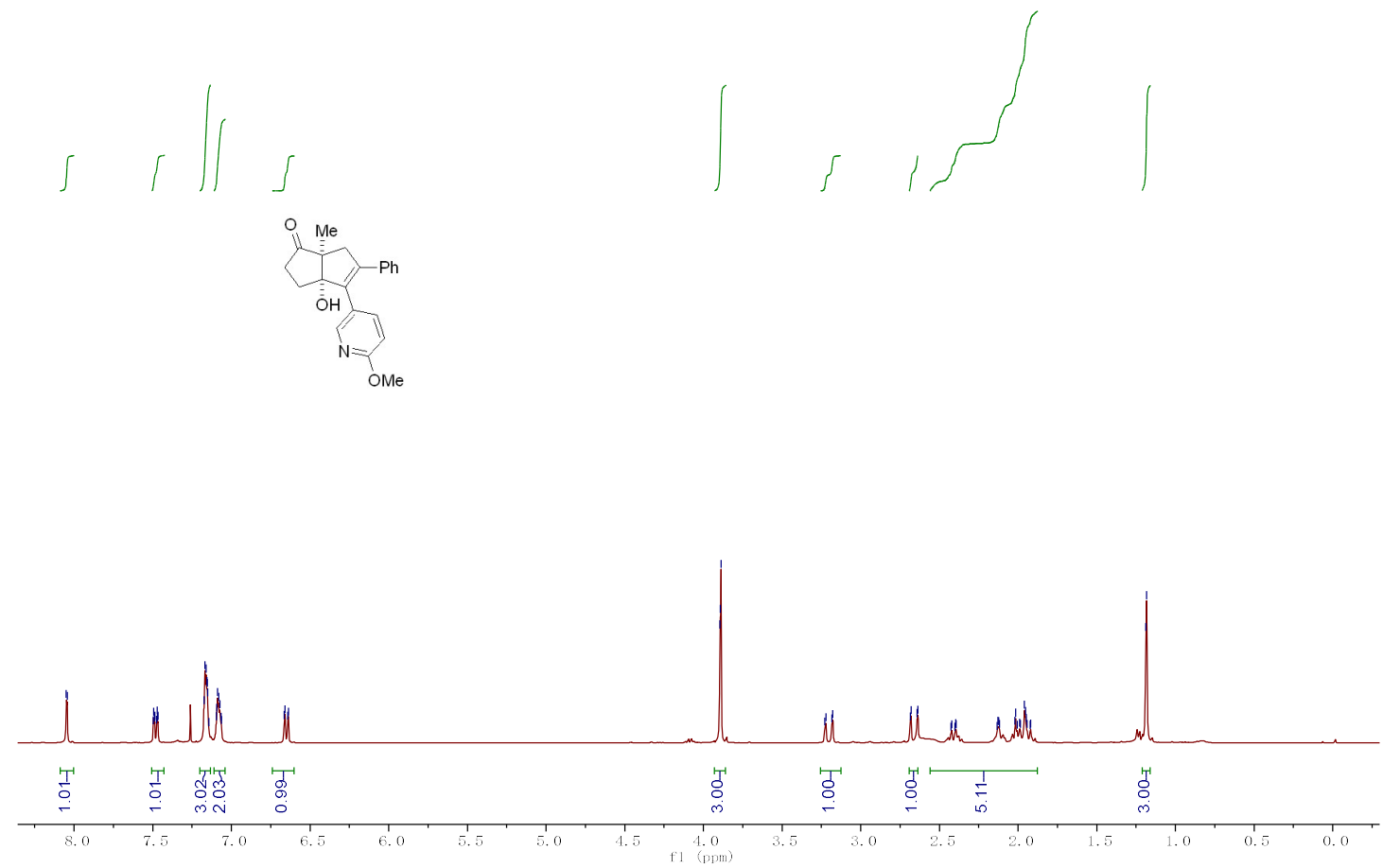

3pa (101 MHz for ${ }^{13} \mathrm{C} \mathrm{NMR}$ with $\mathrm{CDCl}_{3}$ as solvent) $\stackrel{\infty}{\stackrel{n}{n}}$
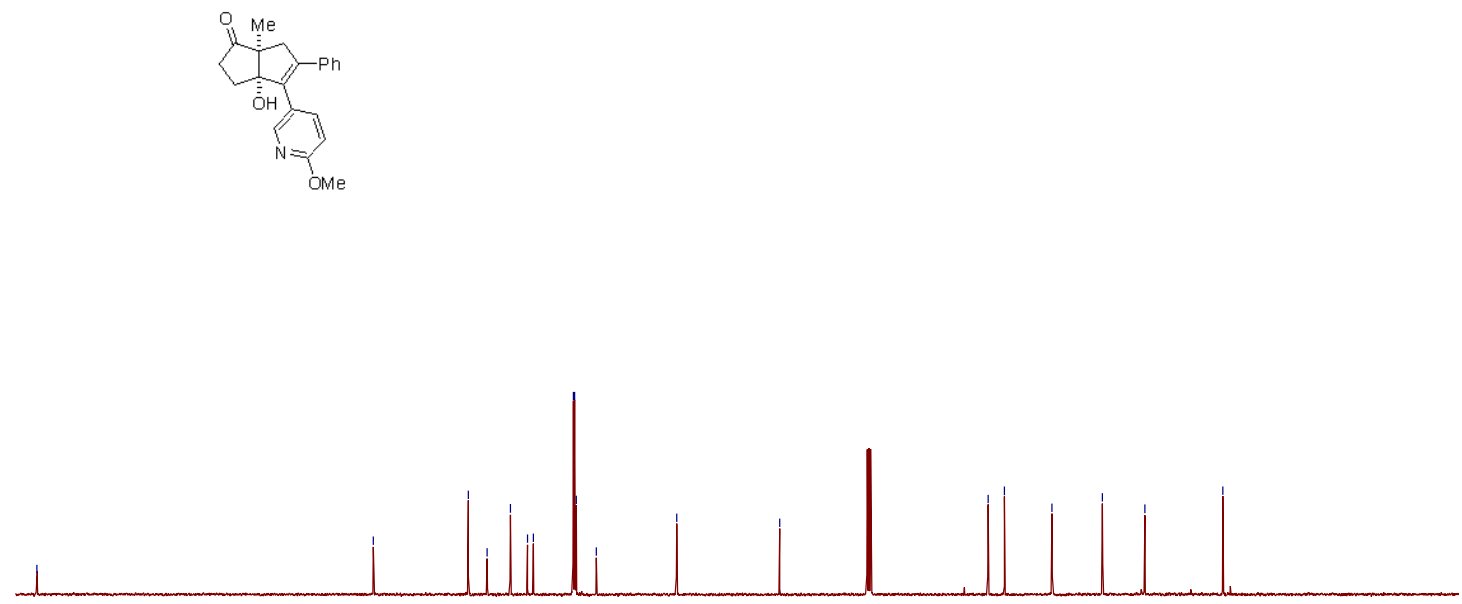
3qa (400 MHz for ${ }^{1} \mathrm{H} \mathrm{NMR}$ with $\mathrm{CDCl}_{3}$ as solvent)

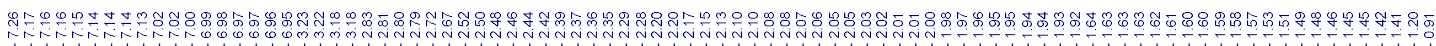
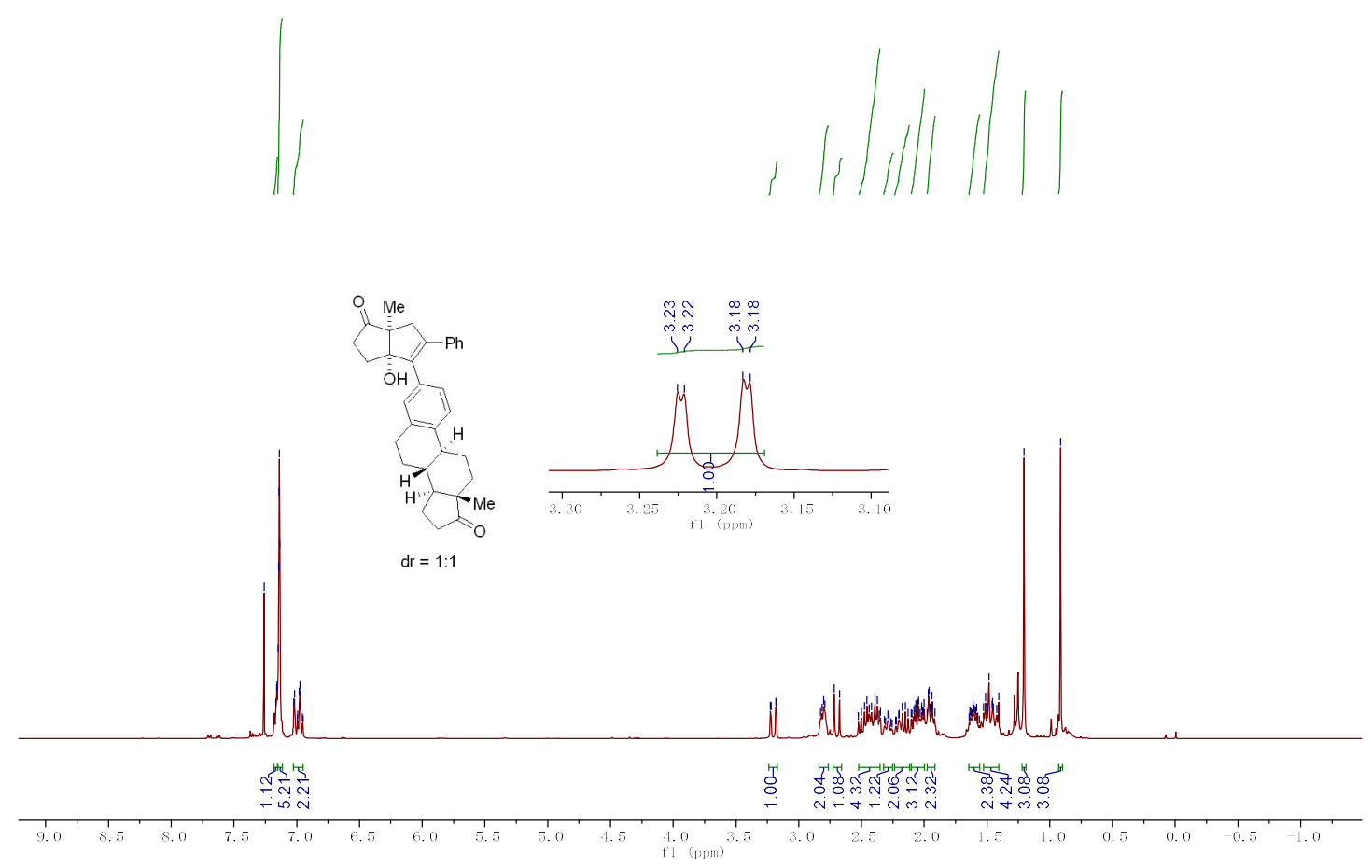

3qa (101 MHz for ${ }^{13} \mathrm{C}$ NMR with $\mathrm{CDCl}_{3}$ as solvent)

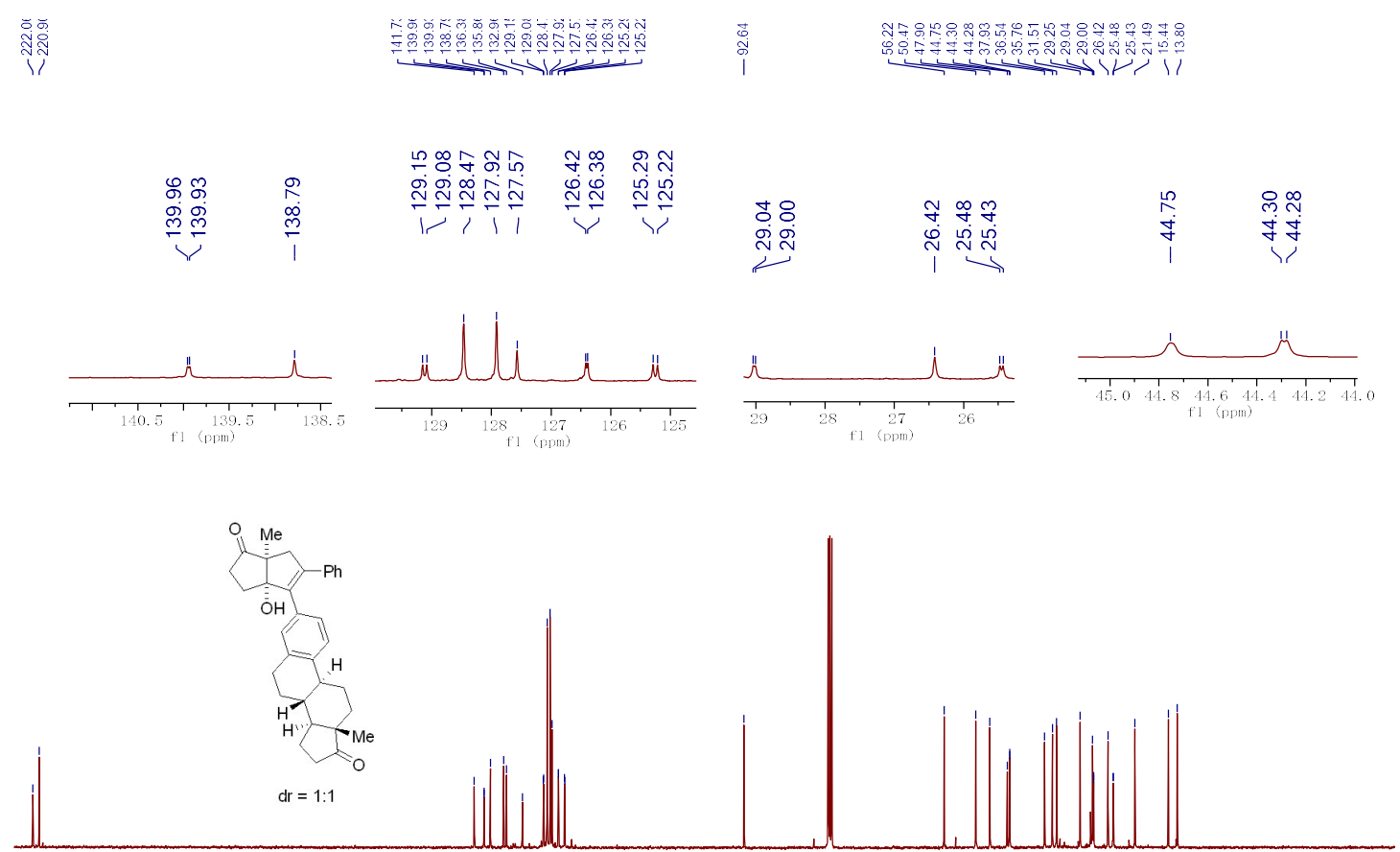


3ra (400 MHz for ${ }^{1} \mathrm{H}$ NMR with $\mathrm{CDCl}_{3}$ as solvent)

盾

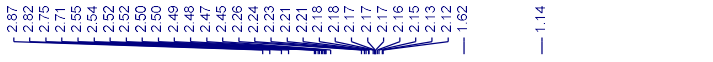<smiles>C1=CC=C1</smiles><smiles>O=C1CCC2C(O)C([As])=C(c3ccccc3)CC12</smiles>

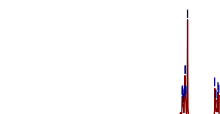

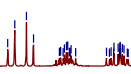

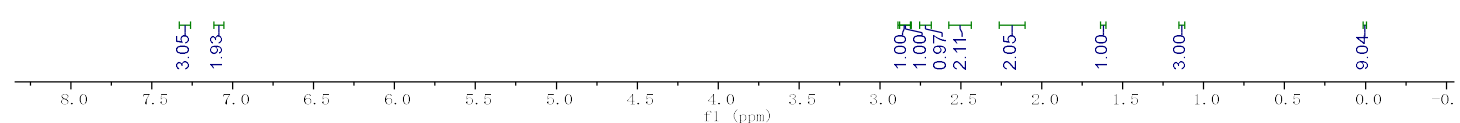

3ra (101 MHz for ${ }^{13} \mathrm{C}$ NMR with $\mathrm{CDCl}_{3}$ as solvent)

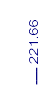
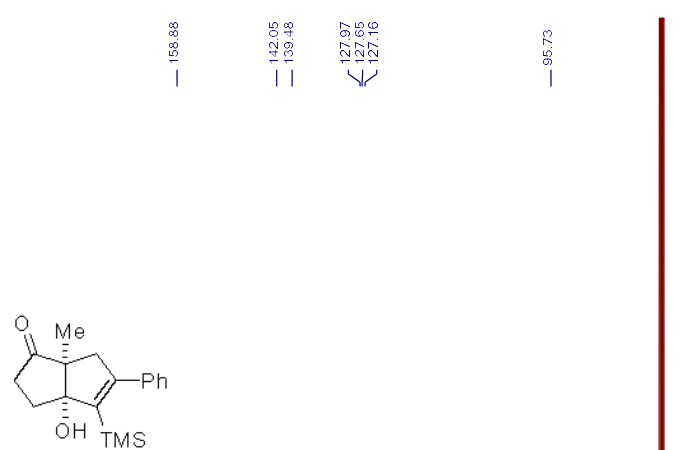
3ua (400 MHz for ${ }^{1} \mathrm{H} \mathrm{NMR}$ with $\mathrm{CDCl}_{3}$ as solvent)

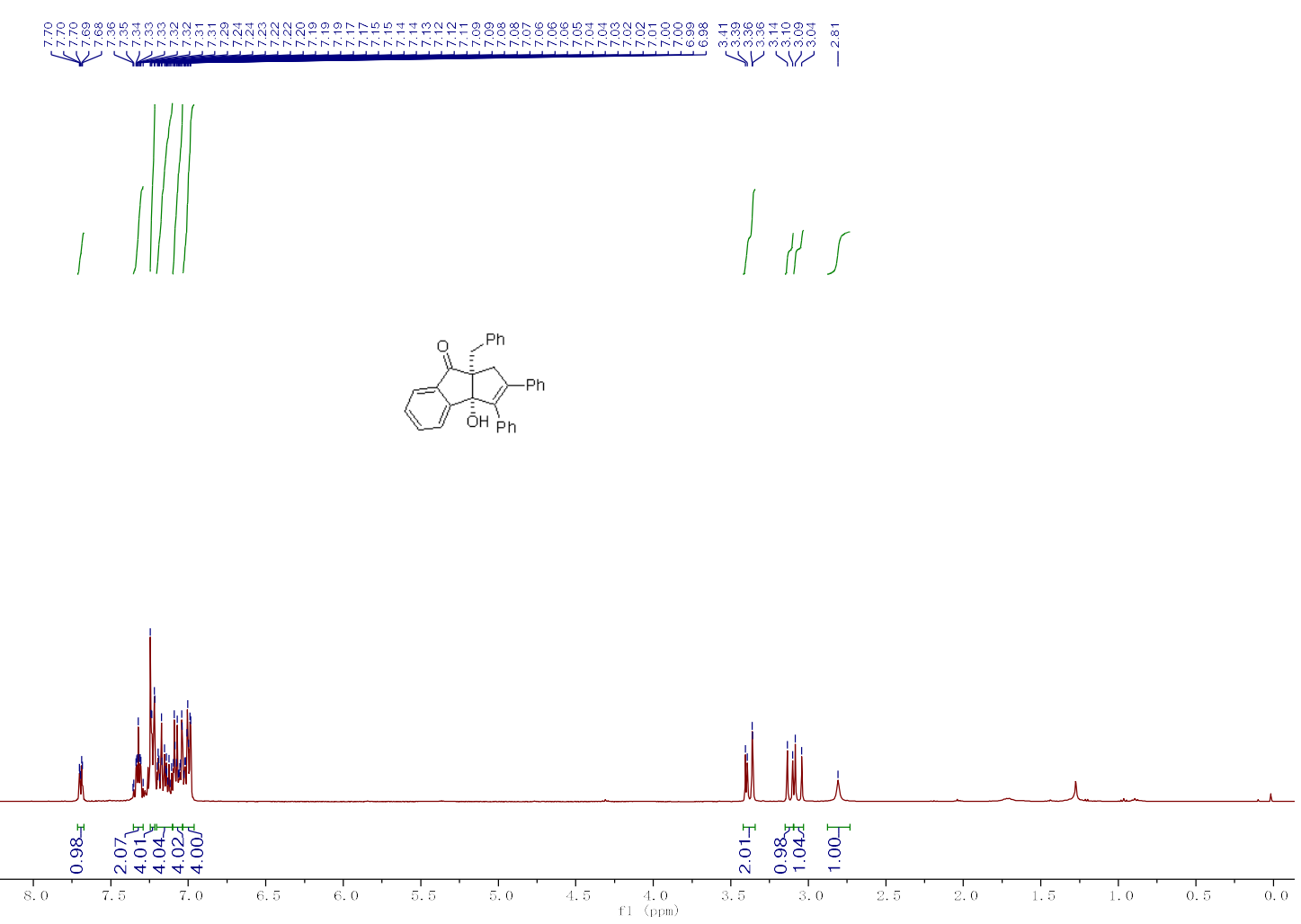

3ua (101 MHz for ${ }^{13} \mathrm{C} \mathrm{NMR}$ with $\mathrm{CDCl}_{3}$ as solvent)

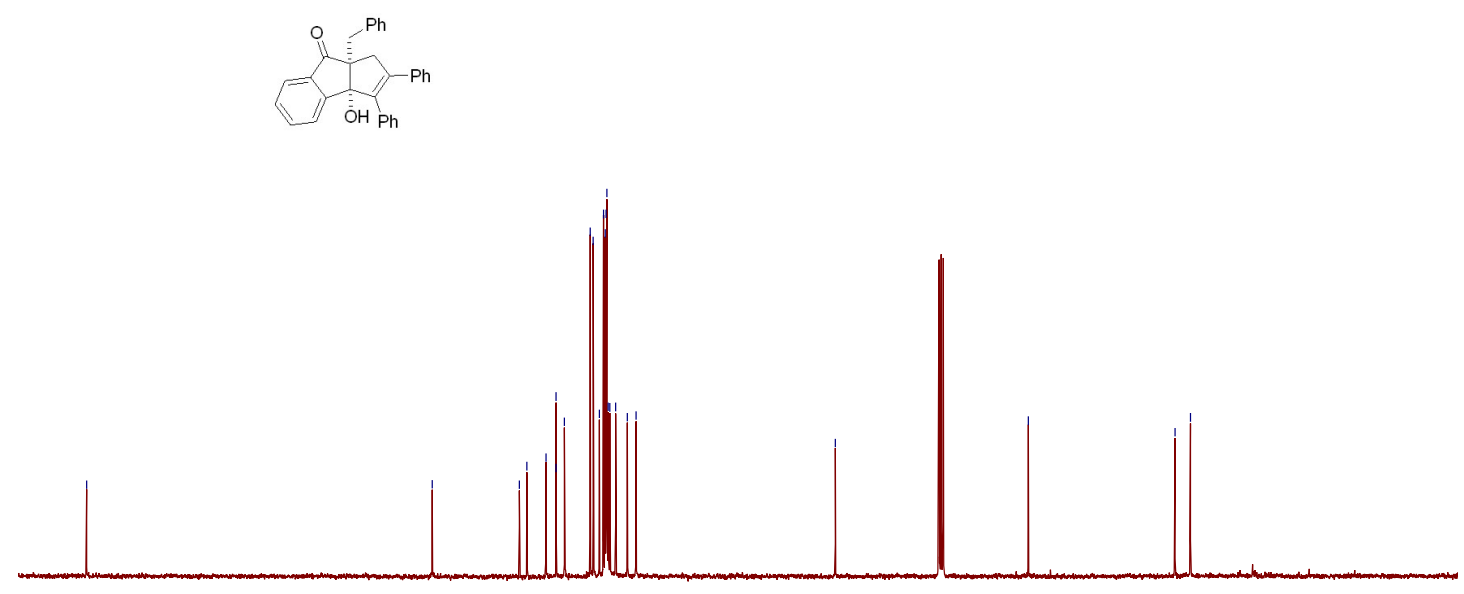


3va (400 MHz for ${ }^{1} \mathrm{H}$ NMR with $\mathrm{CDCl}_{3}$ as solvent)

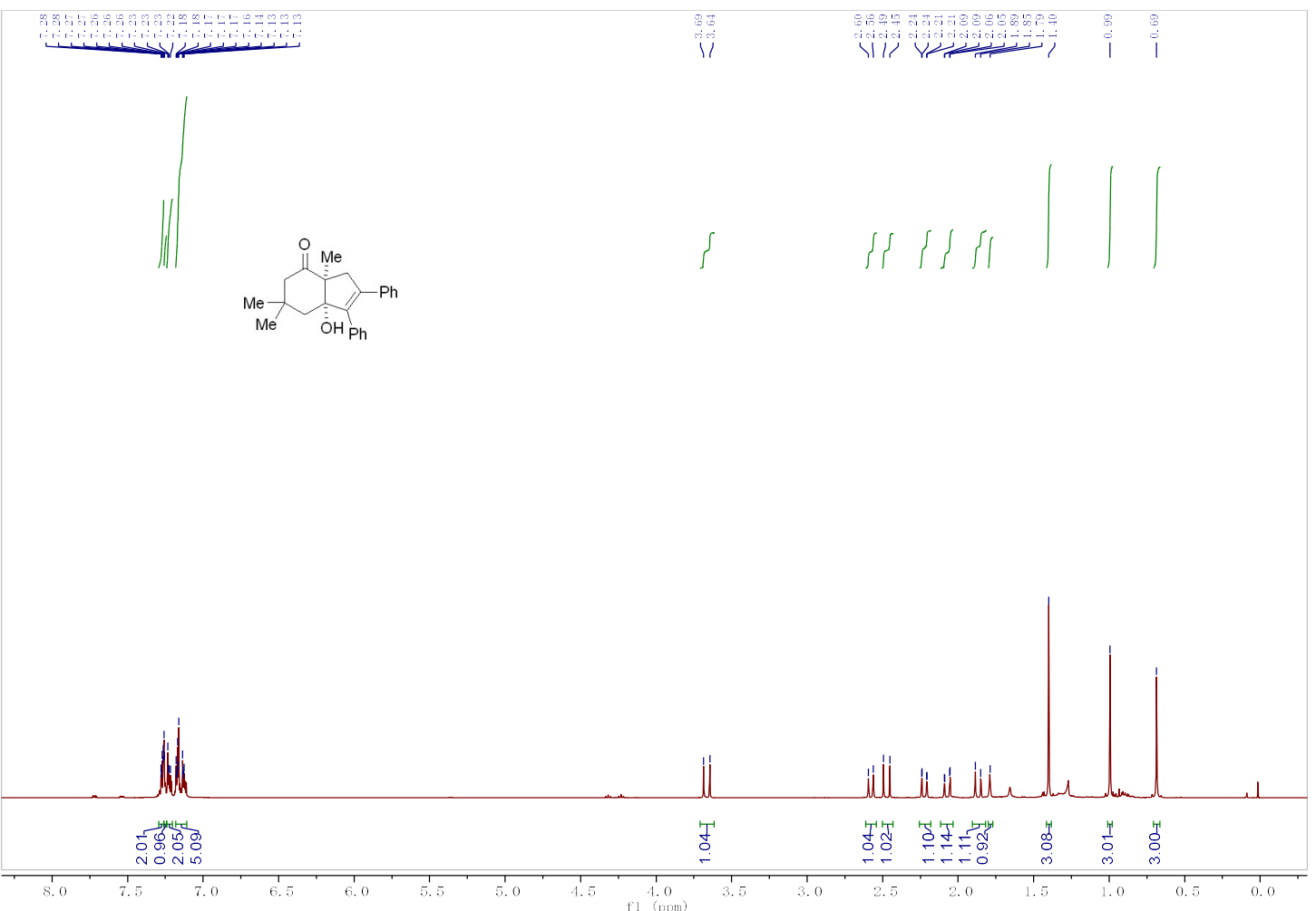

3va (101 MHz for ${ }^{13} \mathrm{C}$ NMR with $\mathrm{CDCl}_{3}$ as solvent)
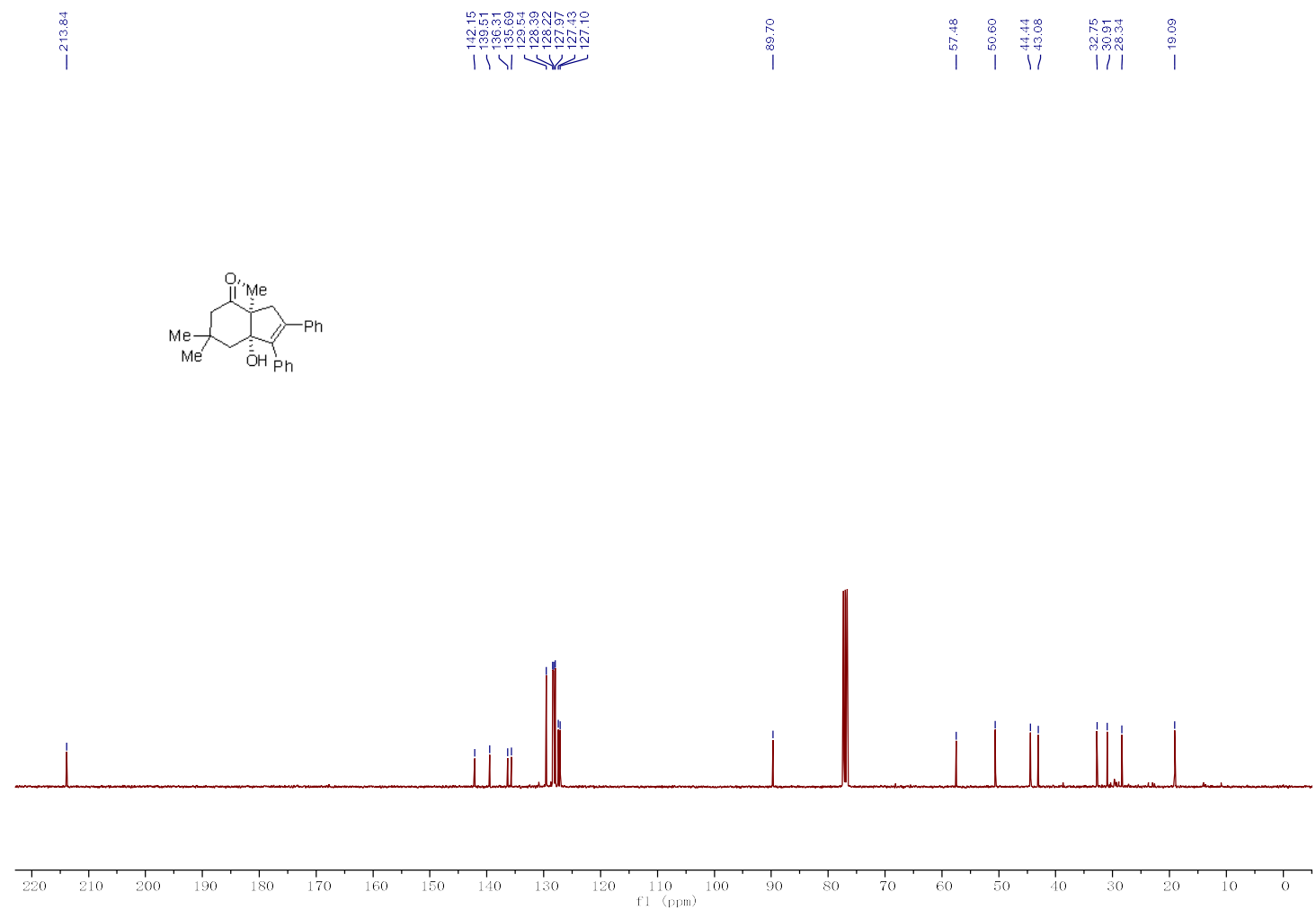
3wa (400 MHz for ${ }^{1} \mathrm{H}$ NMR with $\left(\mathrm{CD}_{3}\right)_{2} \mathrm{CO}$ as solvent)
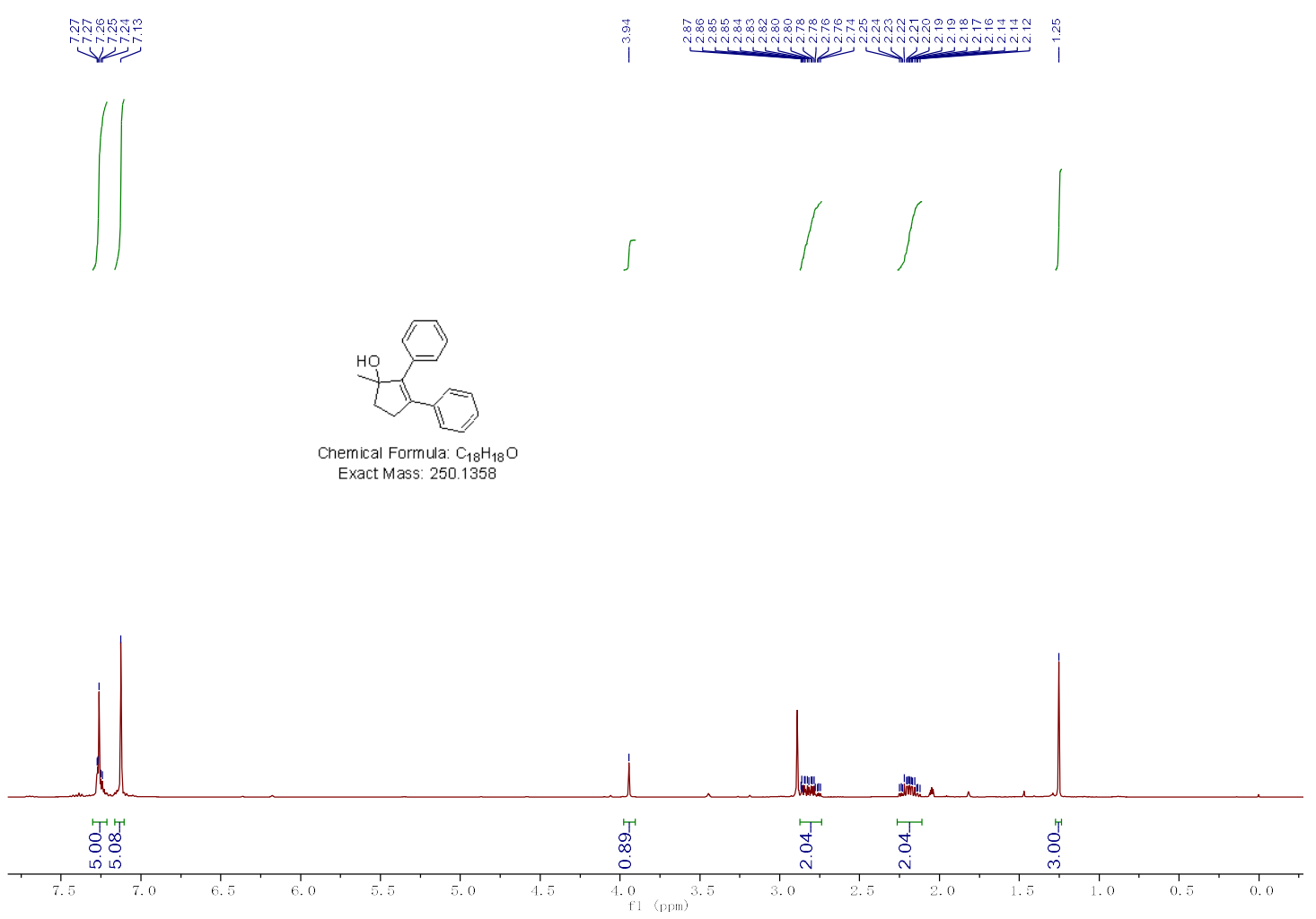

3wa (400 MHz for ${ }^{13} \mathrm{C}$ NMR with $\left(\mathrm{CD}_{3}\right)_{2} \mathrm{CO}$ as solvent)
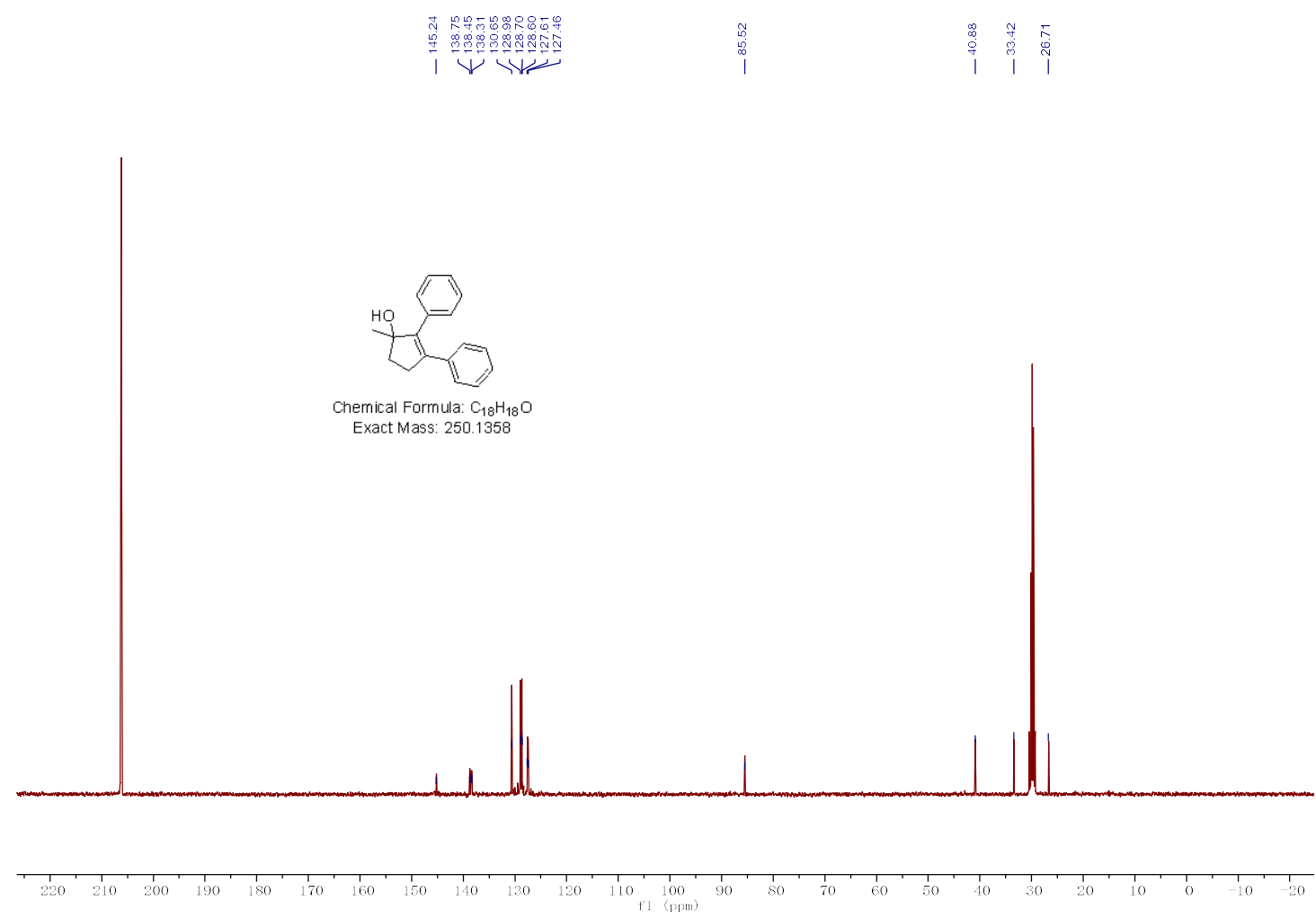

S86 
3xb (600 MHz for ${ }^{1} \mathrm{H}$ NMR with $\left(\mathrm{CD}_{3}\right)_{2} \mathrm{SO}$ as solvent)

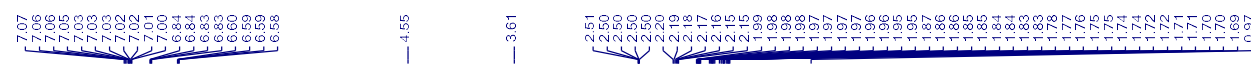

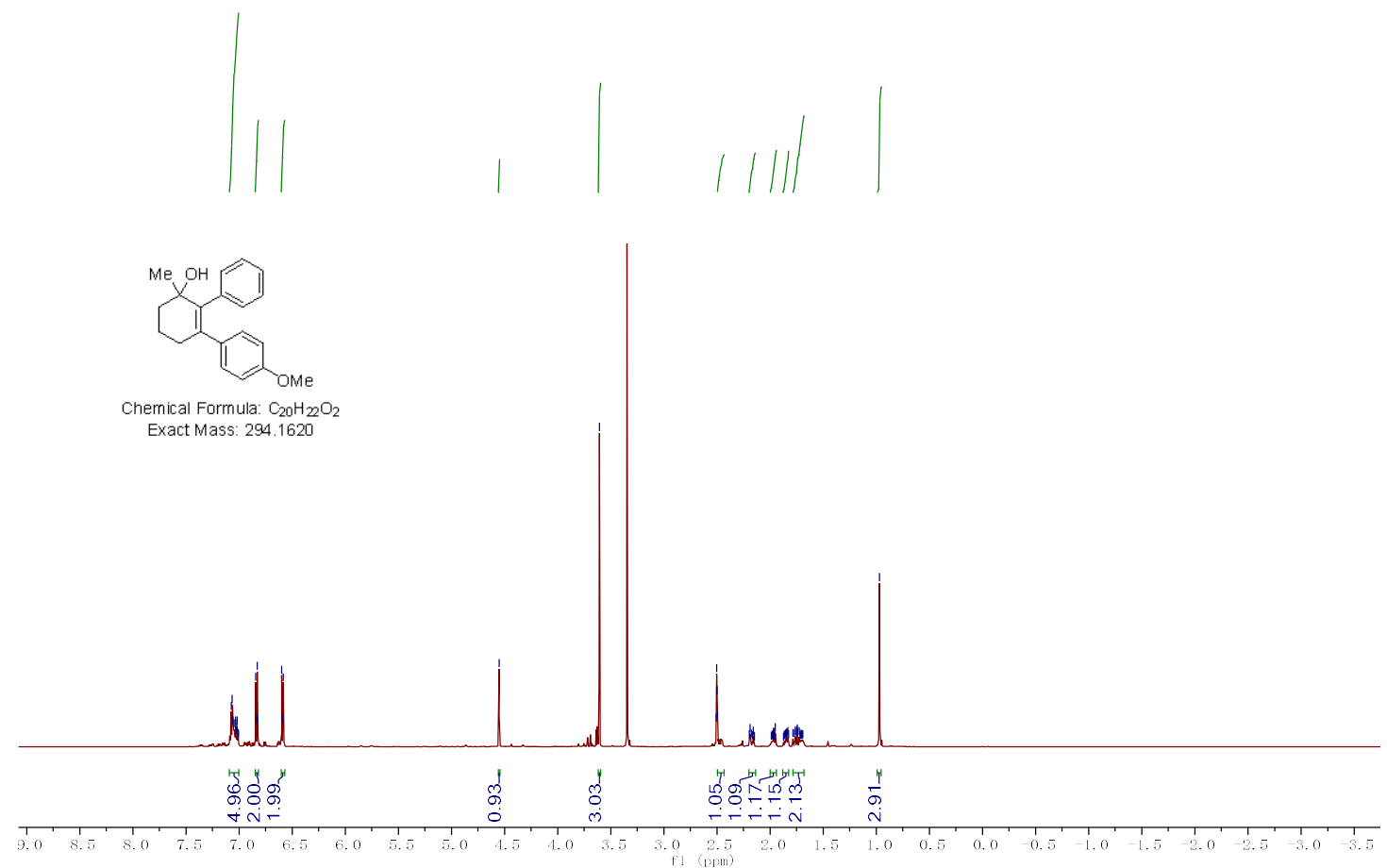

3xb (151 MHz for ${ }^{13} \mathrm{C}$ NMR with $\left(\mathrm{CD}_{3}\right)_{2} \mathrm{SO}$ as solvent)

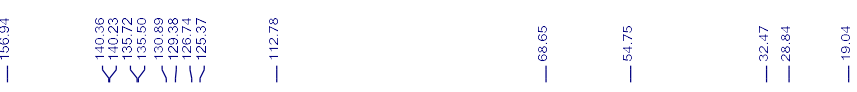

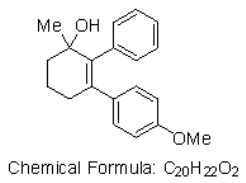

Exat

Exact Mass: 294.1620
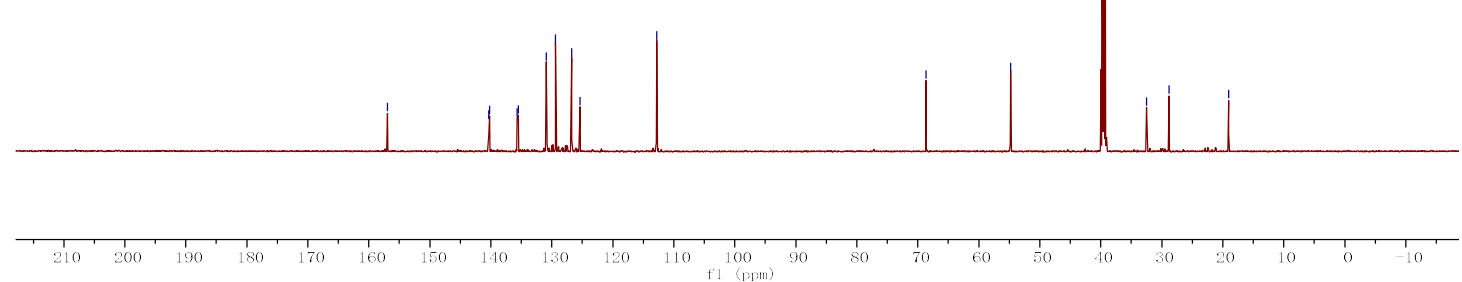
$3 \times \mathbf{x b}(\mathrm{HSQC})$

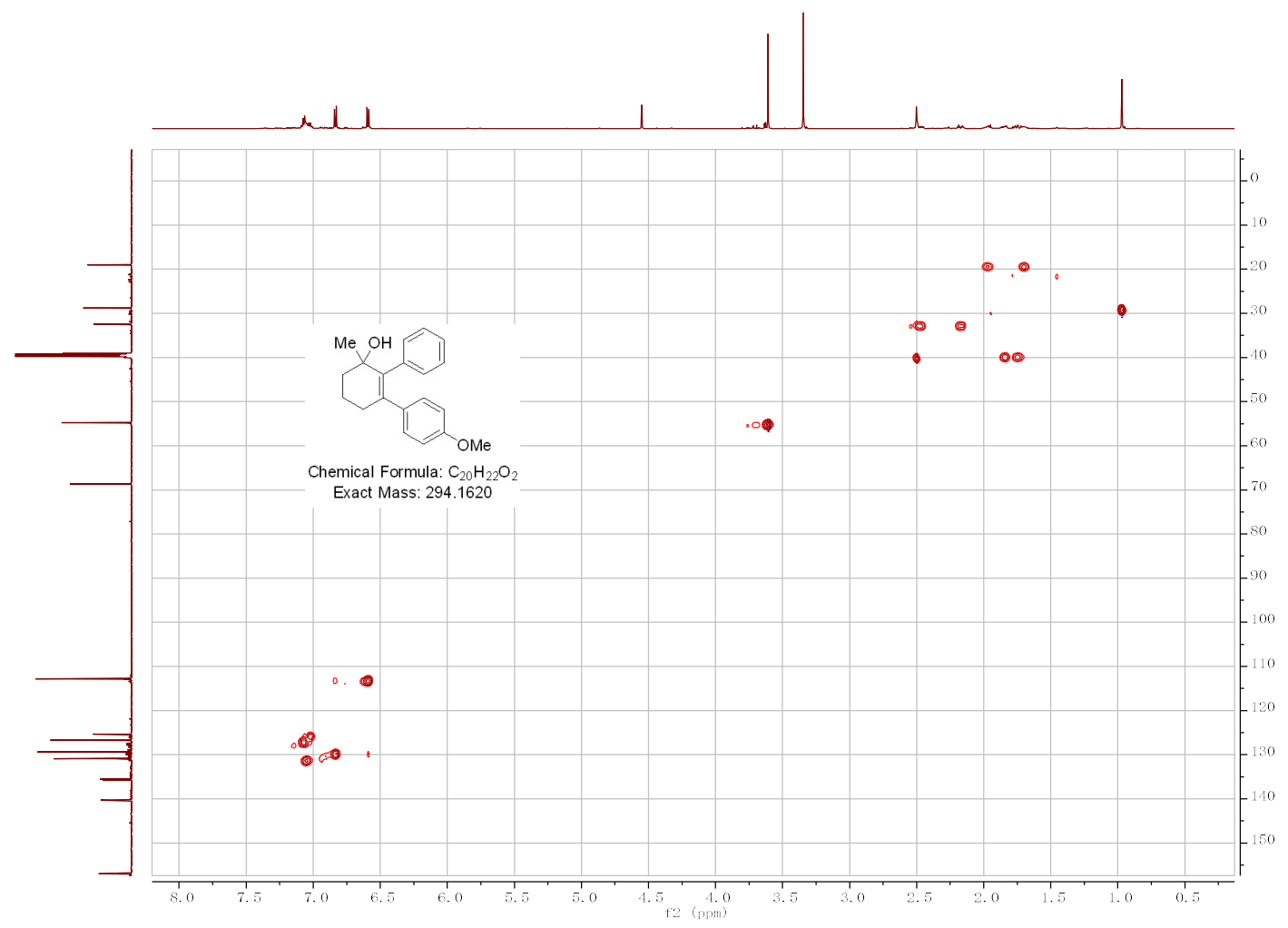

3xb (HMBC)

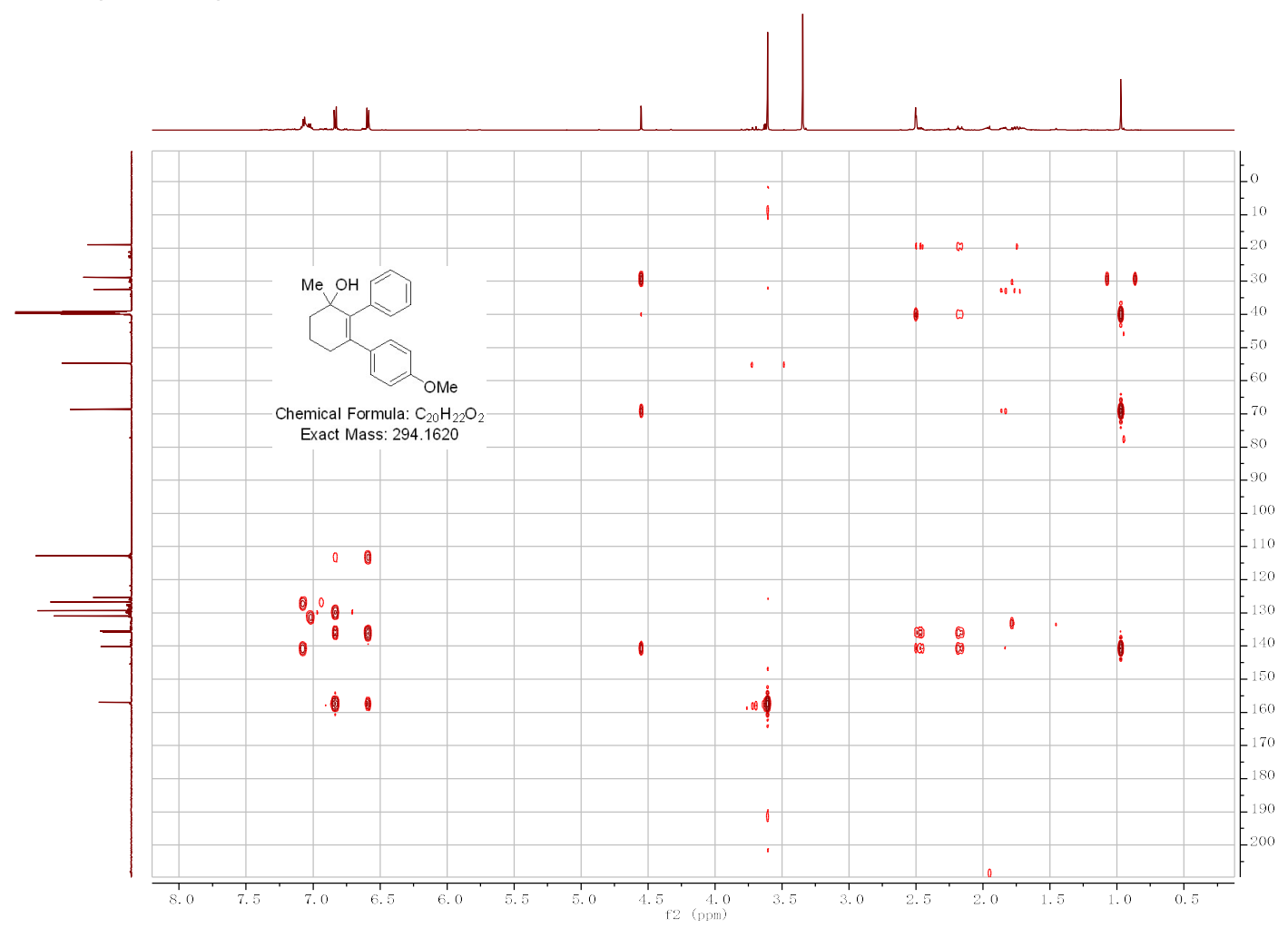


3ya (400 MHz for ${ }^{1} \mathrm{H}$ NMR with $\mathrm{CDCl}_{3}$ as solvent)

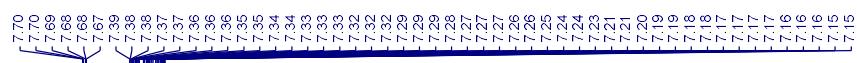

\& || $\mid$

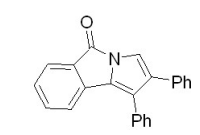

1 illin

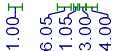

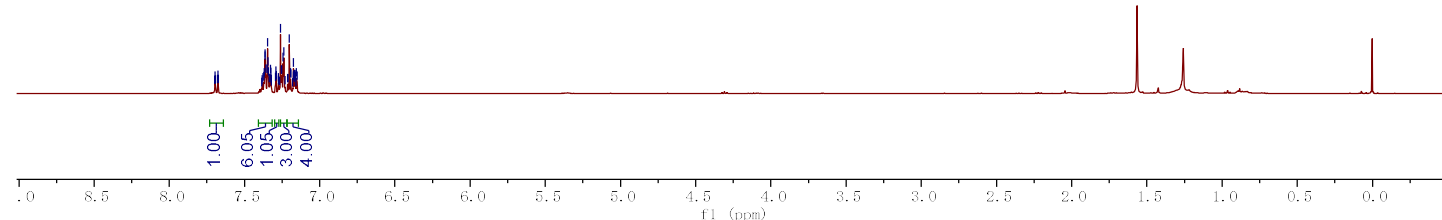

3ya (101 MHz for ${ }^{13} \mathrm{C} \mathrm{NMR}$ with $\mathrm{CDCl}_{3}$ as solvent)
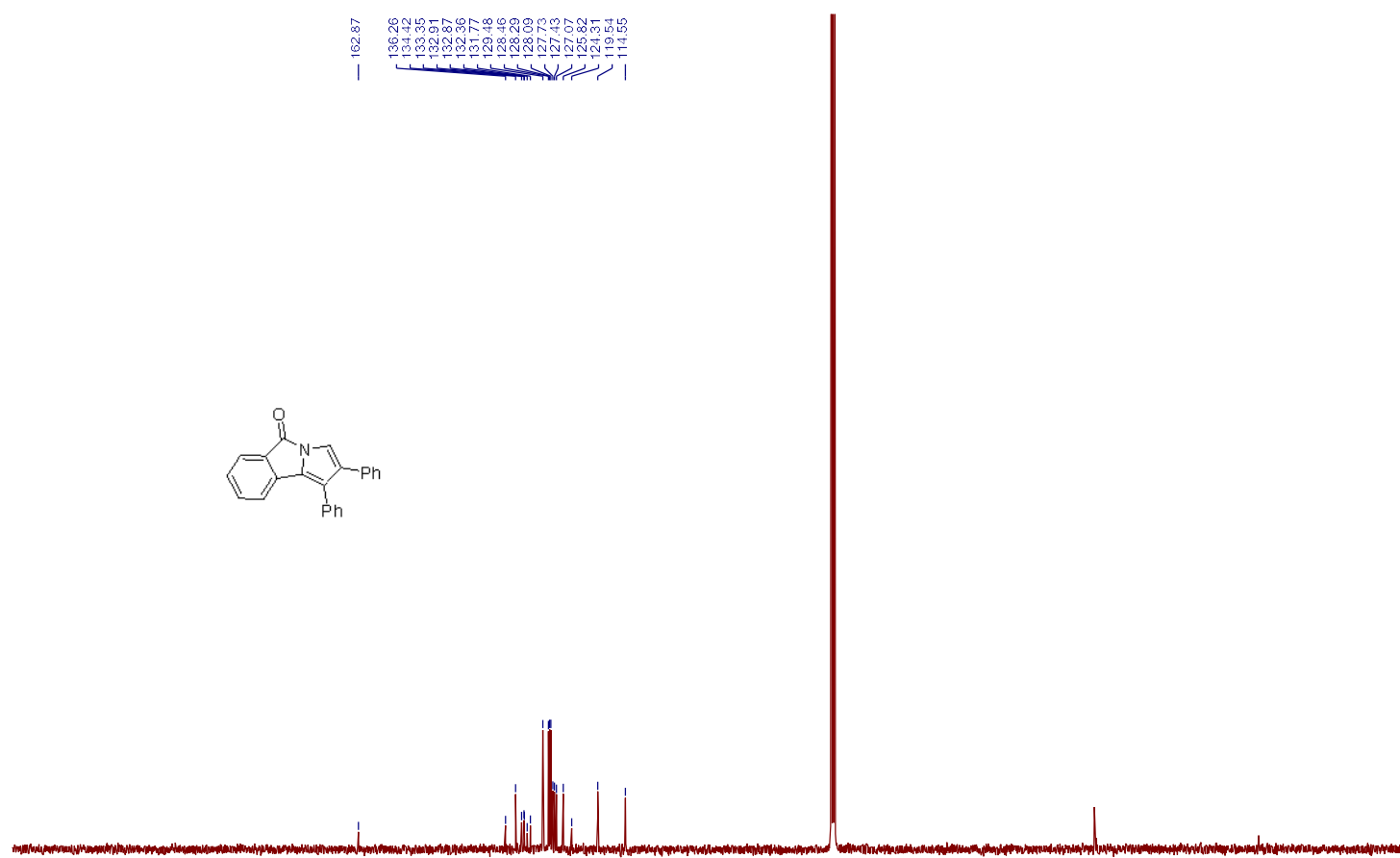


\section{References}

1. Ramachary, D. B.; Kishor, M. Direct amino acid-catalyzed cascade biomimetic reductive alkylations: application to the asymmetric synthesis of Hajos-Parris ketone analogues. Org. Biomol. Chem. 2008, 6, 4176-4187. (d) Kallepu, S.; Gollapelli, K. K.; Nanubol, J. B.; Chegondi, R. Synthesis of highly strained bicyclic [3.n.1] alkenes by a metal-catalyzed Conia-ene reaction. Chem. Commun. 2015, 51, 16840-16843. (c) Cuadros, S.; Dell'Amico, L.; Melchiorre, P. Forging fluorine-containing quaternary stereocenters by a light-driven organocatalytic aldol desymmetrization process. Angew. Chem. Int. Ed. 2017, 56, 11875-11879.

2. (a) Zhu, S.; Zhang, Q.; Chen, K.; Jiang, H. Synergistic catalysis: Metal/protoncatalyzed cyclization of alkynones toward bicyclo[3.n.1]alkanones. Angew. Chem. Int. Ed. 2015, 54, 9414-9418. (b) Clarke, C.; Incerti-Pradillos, C. A.; Lam, H. W. Enantioselective nickel-catalyzed anti-carbometallative cyclizations of alkynyl electrophiles enabled by reversible alkenylnickel E/Z isomerization. J. Am. Chem. Soc. 2016, 138, 8068-8071.

3. (a) Dooley, J. D.; Chidipudi, S. R.; Lam, H. W. Catalyst-controlled divergent C-H functionalization of unsymmetrical 2-aryl cyclic 1,3-dicarbonyl compounds with alkynes and alkenes. J. Am. Chem. Soc. 2013, 135, 10829-10836. (b) Partridge, B. M.; González, J. S.; Lam, H. W. Iridium-catalyzed arylative cyclization of alkynones by 1,4-iridium migration. Angew. Chem. Int. Ed. 2014, 53, 6523-6527. (c) Gollapelli, K. K.; Kallepu, S.; Govindappa, N.; Nanubolub, J. B.; Chegondi, R. Carbonyl-assisted reverse regioselective cascade annulation of 2-acetylenic ketones triggered by Ru-catalyzed $\mathrm{C}-\mathrm{H}$ activation. Chem. Sci. 2016, 7, 4748-4753.

4. Min, X.-T.; Ji, D.-W.; Zheng, H.; Chen, B.-Z.; Hu, Y.-C.; Wan, B.; Chen, Q.-An. Cobalt-Catalyzed Regioselective Carboamidation of Alkynes with Imides Enabled by Cleavage of $\mathrm{C}-\mathrm{N}$ and $\mathrm{C}-\mathrm{C}$ Bonds. Org. Lett. 2020, 22, 3386-3391. 